\title{
UTERINE REGULATION OF PREIMPLANTATION EMBRYO DEVELOPMENT IN FERTILITY- CLASSIFIED HEIFERS
}

\author{
A dissertation submitted in partial fulfillment of \\ the requirements for the degree of \\ DOCTOR OF PHILOSOPHY IN ANIMAL SCIENCES \\ UNIVERSITY OF MISSOURI- COLUMBIA \\ Division of Animal Science
}

By

JOAO GABRIEL NASCIMENTO MORAES

Dr. Thomas Spencer, Dissertation Supervisor December 2018 
The undersigned have examined the dissertation entitled,

\section{UTERINE REGULATION OF PREIMPLANTATION EMBRYO DEVELOPMENT IN FERTILITY-CLASSIFIED HEIFERS}

presented by Joao Gabriel Nascimento Moraes,

a candidate for the degree of doctor of philosophy, and hereby certify that, in their opinion, it is worthy of acceptance.

Chair, Dr. Thomas Spencer

Dr. Thomas W Geary

Dr. Michael F. Smith

Dr. Matthew C. Lucy

Dr. Scott Poock 


\section{ACKNOWLEDGEMENTS}

First, I would like to express my sincere gratitude to my adviser, Dr. Thomas Spencer, for his guidance, encouragement and advice throughout my doctoral program. Thank you for supporting my research and for allowing me to grow as a scientist. I feel very lucky for having had the opportunity of joining the 'Team Uterus' and work alongside great people on outstanding projects. I also would like to thank the current and past members from Dr. Spencer's lab for all their help and friendship during the last four years: Sofia Ortega, Andrew Kelleher, Greg Burns, Kelsey Brooks, Eleanore O’Neil, Carolyn Allen, Pramod Dhakal, Wang Peng, Brenda Jesernig, Jessica Foster, Harriet Fitzgerald, and Linda Rowland.

My sincere thanks must go also to the members of my dissertation committee, Dr. Thomas Geary, Dr. Michael Smith, Dr. Matthew Lucy and Dr. Scott Poock. I am grateful for having joined Mizzou during the second year of my $\mathrm{PhD}$ program and proud to have this great group of scientists in my committee. Thank you for offering me several learning opportunities, for making me a part of your projects, and for your support to my future career as an independent scientist.

I also would like to acknowledge the groups of Dr. Holly Neibergs at Washington State University, Dr. Peter Hansen at the University of Florida, and Dr. Thomas Hansen at Colorado State University for their collaboration on these projects. Also, I want to express my appreciation to the students, faculty members and staff of University of Missouri and Washington State University for their assistance during my doctoral program. I am especially indebted to Dr. Susanta Behura for all his support with bioinformatics, to Dr. William R. Lamberson for teaching me so much about statistics, to Kenneth Ladyman and David Todd for their help at the beef farm, and to Rick Disselhorst and the meats lab crew for their assistance with animal slaughter. 
Last but not least, I would like to express my deepest gratitude to my family and friends for their love and support during every step of the way. 


\section{Table of Contents}

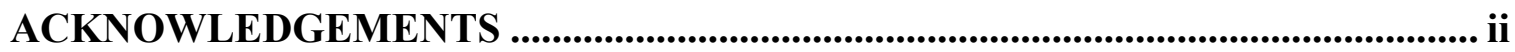

LIST OF FIGURES AND TABLES......................................................................... vii

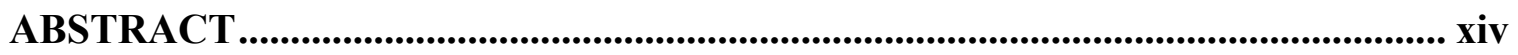

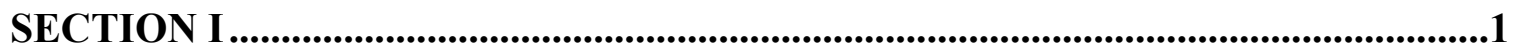

LITERATURE REVIEW ....................................................................................................1

Overview of pregnancy loss in cattle .....................................................................................1

Early pregnancy development in ruminants .....................................................................8

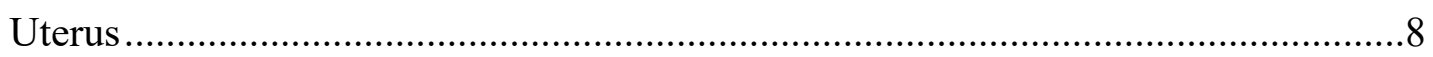

Preimplantation embryonic development ………………….................................11

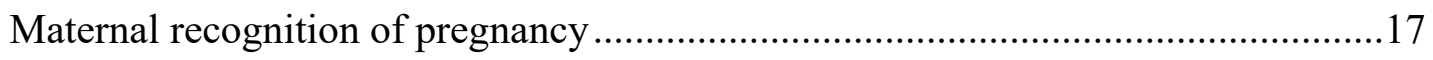

Histotroph components ...........................................................................................................25

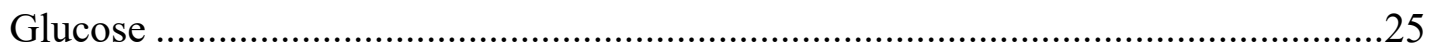

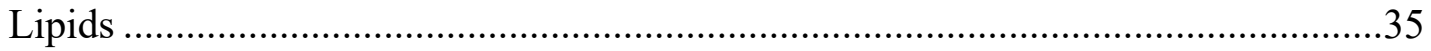

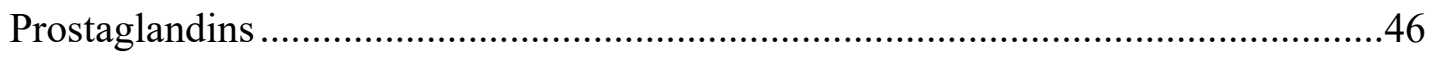

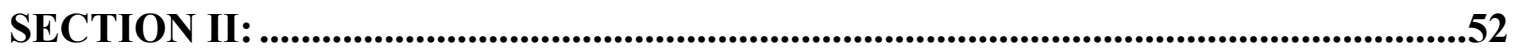

Identification of Beef Heifers with Superior Uterine Capacity for Pregnancy ...........52

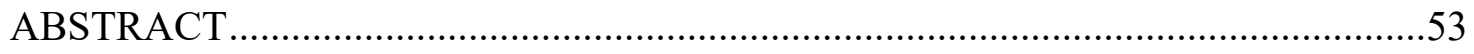

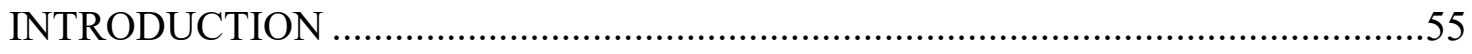




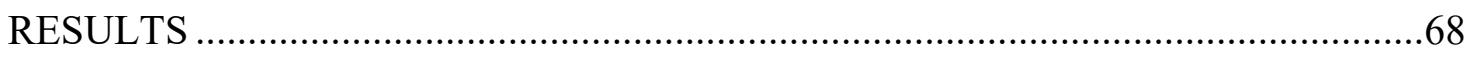

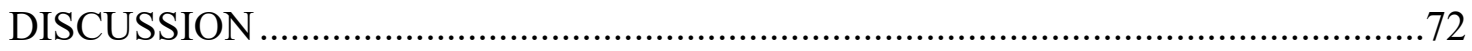

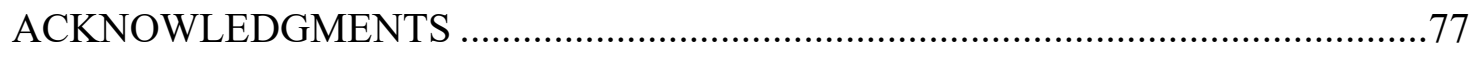

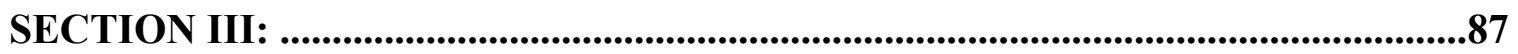

Uterine Influences on Conceptus Development in Fertility-Classified Animals .........87

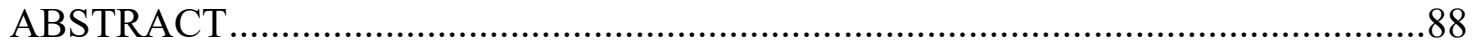

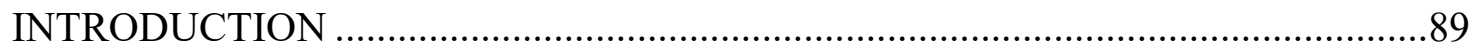

MATERIALS AND METHODS....................................................................... 91

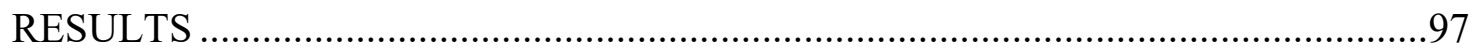

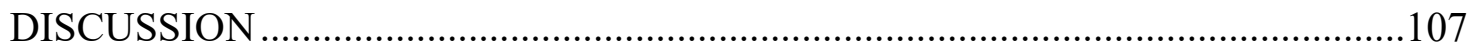

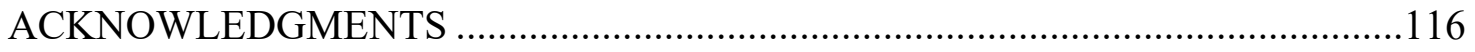

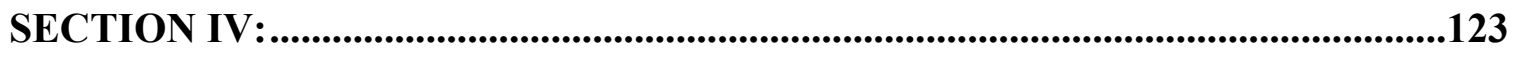

Analysis of the Uterine Lumen from Fertility-Classified Heifers: I. Glucose,

Prostaglandins and Lipids...........................................................................................123

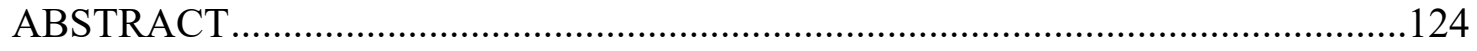

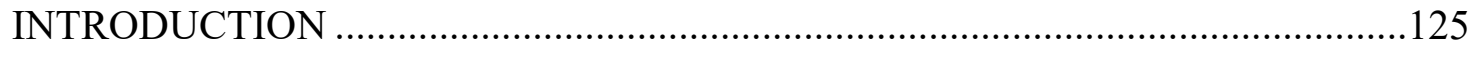

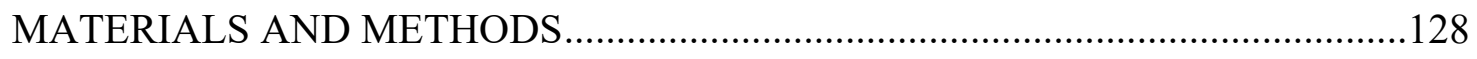

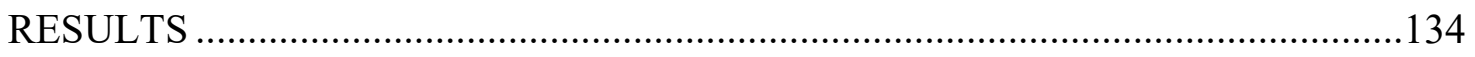

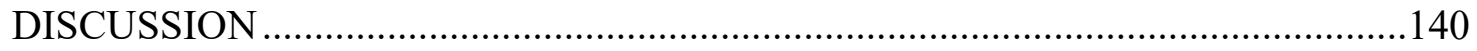


SECTION V:

Analysis of the Uterine Lumen from Fertility-Classified Heifers: II Interferon-tau,

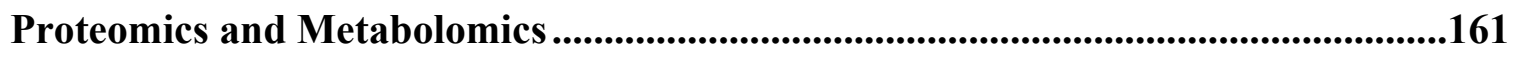

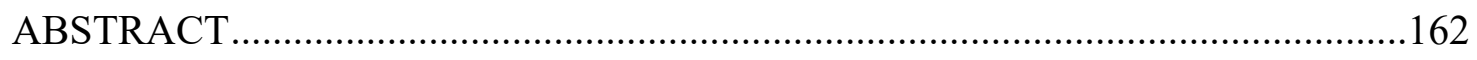

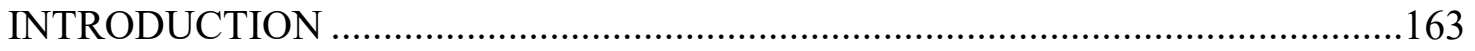

MATERIALS AND METHODS ........................................................................ 166

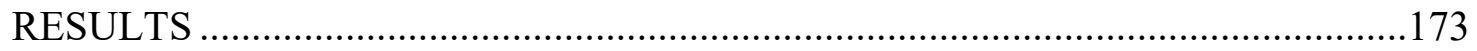

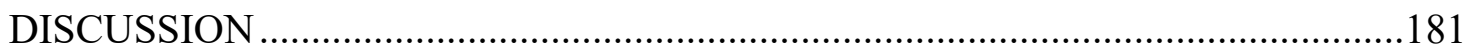

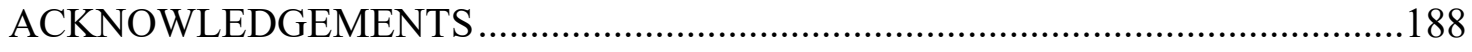

CONCLUSIONS AND FUTURE DIRECTIONS.......................................................203

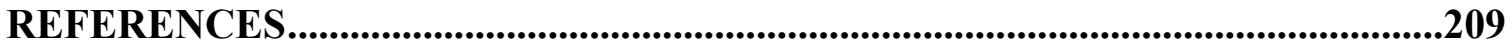

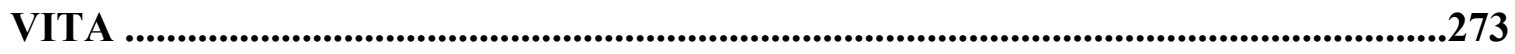




\section{LIST OF FIGURES AND TABLES}

\section{SECTION I:}

\section{FIGURES}

Figure 1. Proposed rate of pregnancy loss from ovulation through calving in beef heifers.

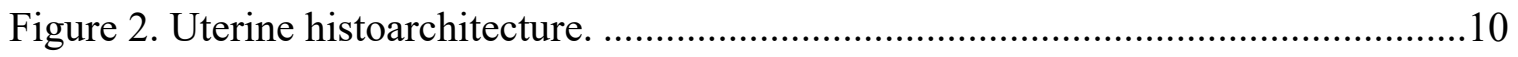

Figure 3. Early embryonic development in cattle from ovulation to attachment. .............14

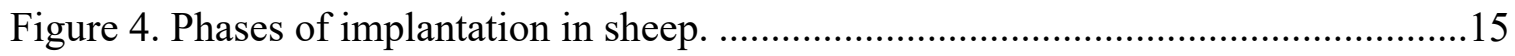

Figure 5. Regulation of the estrus cycle in pregnant and nonpregnant ewes...................20

Figure 6. Conserved transcription factor biding sites on a variety of interferon tau (IFNT)

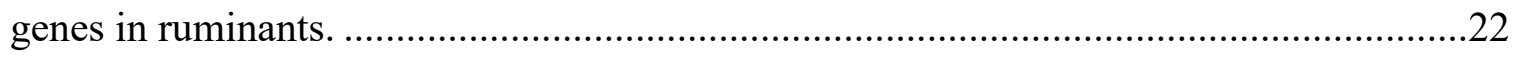

Figure 7. Suggested model of transcriptional control of interferon tau (IFNT) expression

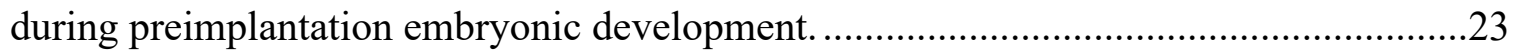

Figure 8. Transport of glucose through glucose transporters. .....................................30

Figure 9. Glucose and fatty acid metabolism in cancer cells....................................... 31

Figure 10. Major metabolic pathways involved with glutamine metabolism...................32

Figure 11. Glutamine metabolism and regulation of oxidative stress. ...........................33

Figure 12. Activation of the mechanistic target of rapamycin complex 1 (mTORC1) by

glutamine and leucine.

Figure 13. Lipid accumulation in the uterine lumen and within endometrial epithelial

cells.

Figure 14. Mechanism of transcriptional activation of peroxisome proliferator-activated

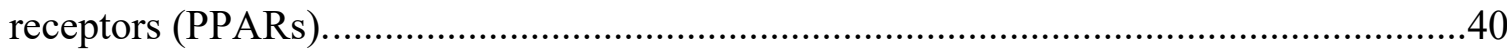

Figure 15. Mitochondrial fatty acid $\beta$-oxidation....................................................41 
Figure 16. Fatty acid elongation and desaturation.

Figure 17. Proposed model for lipid metabolism during early pregnancy in cattle..........44

Figure 18. Prostaglandin synthesis from membrane phospholipids.

Figure 19. Proposed model for prostaglandin (PG) and interferon tau (IFNT) regulation of uterine function and conceptus elongation during early pregnancy in sheep.

\section{TABLE}

Table 1. Pregnancy loss in lactating dairy cows during the first week following artificial

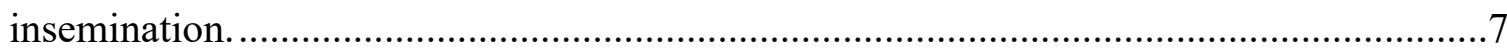




\section{SECTION II:}

\section{FIGURES}

Figure 1. Experimental design and results for classification of fertility in beef heifers

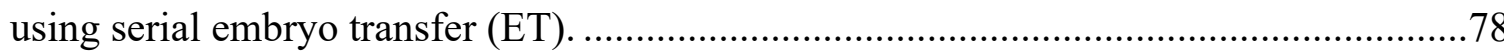

Figure 2. Circulating concentrations of progesterone in nonpregnant (A) and pregnant (B)

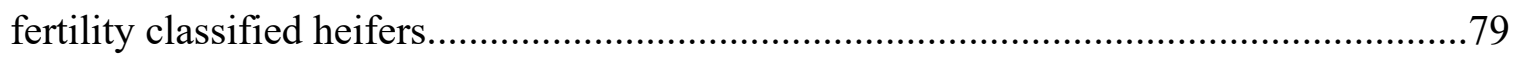

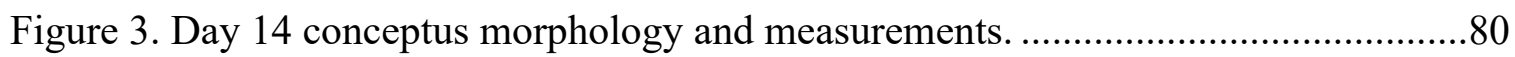

Figure 4. Endometrial biopsy histology and RNA sequencing analysis from day 14

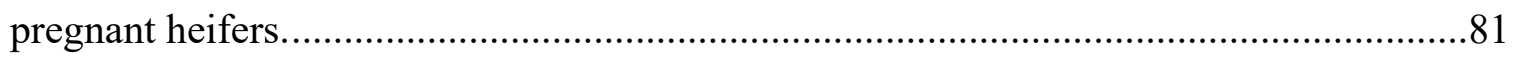

Figure 5. Venn diagram for differently expressed genes in the endometrium of pregnant

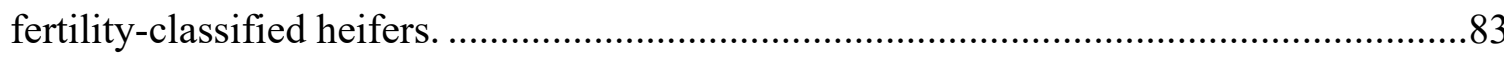

\section{TABLES}

Table 1. Differentially expressed genes in endometrial biopsies from high fertile (HF) and subfertile (SF) heifers.

Table 2. Differentially expressed genes in endometrial biopsies from subfertile (SF) and

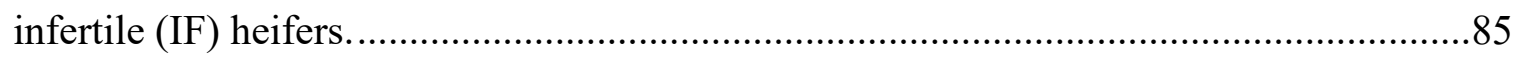

Table 3. Differentially expressed genes in endometrial biopsies from high fertile (HF) and infertile (IF) heifers 


\section{SECTION III:}

\section{FIGURES}

Figure 1. Day 17 pregnancy outcome in fertility-classified heifers..............................117

Figure 2. Summary of endometrium and conceptus transcriptome analyses.................119

Figure 3. Transcriptome analysis of endometrium from nonpregnant and pregnant day 17

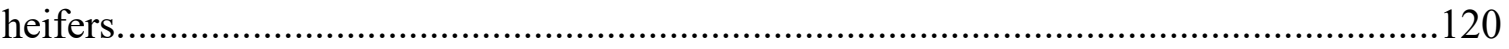

Figure 4. Receptor-ligand interactions between conceptus and endometrium at day 17 of

pregnancy in high fertile (HF) and subfertile (SF) heifers. 


\section{SECTION VI:}

\section{FIGURES}

Figure 1. Glucose concentrations in the ULF and plasma of fertility classified heifers..149

Figure 2. Correlations between ULF glucose, plasma glucose and conceptus size.........150

Figure 3. Concentrations of prostaglandins in the ULF of fertility-classified heifers.....151

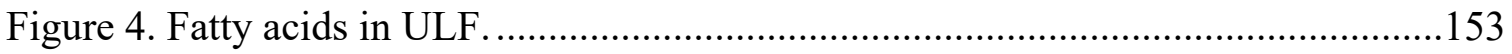

Figure 5. Heatmap for fatty acid concentration in the ULF across samples....................154

\section{TABLES}

Table 1. Expression of genes in the endometrium involved with glucose transport .......155 Table 2. Differently expressed genes in the endometrium of fertility-classified heifers for genes involved with glycolysis and gluconeogenesis using KEGG database 156

Table 3. Expression of genes involved with glycolysis and gluconeogenesis in conceptuses from fertility-classified heifers using the KEGG database

Table 4. Expression of selected genes involved with prostaglandins synthesis or signaling

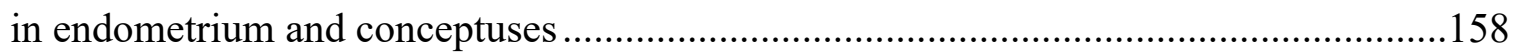

Table 5. Fatty acid differences in the ULF of fertility-classified heifers.........................159

Table 6. Lipids differentially abundant in the ULF of open and pregnant HF heifers ....160 


\section{SECTION V:}

\section{FIGURES}

Figure 1. Concentrations of interferon tau in the ULF of fertility-classified heifers.......189 Figure 2. Venn diagram for proteins differently abundant in the ULF of pregnant HF and SF heifers according to the SDS-PAGE procedure. 190

Figure 3. Venn diagram showing the differently abundant proteins among open fertilityclassified heifers

Figure 4. Selected genes/proteins hypothesized to be regulated by pregnancy based on combined data from endometrium transcriptome and ULF proteome.

Figure 5. Metabolite set enrichment analysis (MSEA).

Figure 6. Joint pathway analysis for endometrium transcriptome and ULF metabolome.

\section{TABLES}

Table 1. Expression of interferon tau (IFNT) by conceptuses and of selected classical and nonclassical interferon stimulated genes by the endometrium .196

Table 2. Proteins differentially abundant in ULF by method.

Table 3. Top 10 proteins that increased or decreased in pregnant compared to open ULF

Table 4. Summary of the differential metabolites identified in the ULF.

Table 5. Top 20 identified metabolites with the highest differences in fold change in ULF of pregnant than to open heifers. 
Table 6. Top 20 identified metabolites with the highest differences in fold change in ULF

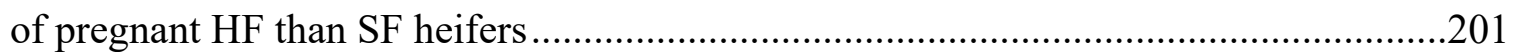

Table S1. Expression of selected genes involved with the transcriptional control of IFNT expression .202 


\begin{abstract}
Infertility and subfertility represent pervasive problems in domestic animals and humans, and embryonic mortality is a major factor influencing reproductive efficiency. In cattle, the majority of embryonic loss occurs during the first month of gestation that involves the period of blastocyst formation, conceptus elongation, maternal recognition of pregnancy, implantation and beginning of placentation. Pregnancy success and embryonic mortality are affected by paternal, maternal, embryonic, and environmental factors, and the establishment and maintenance of pregnancy are a result of complex conceptusendometrium interactions that results in adequate conceptuses (embryo/fetus and associated extraembryonic membranes) development, implantation and placentation. Our central hypothesis is that the uterus directly influences embryonic and conceptus development, and we proposed that heifers with consistently high or low fertility have distinct uterine capacity to support pregnancy. To test this hypothesis, serial embryo transfer (3-4 rounds) was used to classify heifers based on pregnancy success on day 28 as high fertile (HF; 100\%), subfertile (SF; 25\%), or infertile (IF; 0\%). Next, a series of experiments were conducted using the fertility-classified heifers to investigate conceptus development and uterine biology in two time points: (1) day 14, to investigate conceptus development prior to the period when pregnancy induce changes are detected in the endometrium transcriptome; (2) at day 17, to evaluate conceptus-endometrial cross talk during the period of maternal recognition of pregnancy. Results from the studies conducted on day 14 supports the idea that: (1) circulating progesterone concentrations are not different among fertility-classified heifers; (2) conceptus development and survival by day 14 is not affected by fertility classification; (3) only minimal differences in endometrium transcriptome are detected among pregnant fertility-classified heifers. Collectively, these
\end{abstract}


results indicated that the biological mechanisms underlying subfertility and infertility manifests between days 14 and 28, when pregnancy recognition signaling and conceptus elongation and implantation must occur for the establishment of pregnancy. Moreover, results from the subsequent experiments conducted at day 17 indicated that: (1) the mechanism of pregnancy loss in fertility-classified heifers start to manifest around the time of maternal recognition of pregnancy; (2) conceptus survival by day 17 is compromised in IF heifers; (3) conceptus development is advanced in HF than SF heifers; (4) conceptus transcriptome is directly influenced by the uterine environment; (5) dysregulated conceptus-endometrial interactions in SF heifers seems to be the major cause of pregnancy loss. Analysis of the uterine luminal fluid (ULF) from fertility classified heifers on day 17 established that: (1) ULF composition is affected by conceptus-endometrium interactions; (2) glucose concentrations in ULF are not different among fertility-classified heifers; (3) pregnancy induced changes in the metabolites found in ULF was diminished in SF heifers, and the majority of the metabolites that increased in the ULF of pregnant HF than SF heifers were associated with energy and amino acid metabolism; (4) increased abundance of proteins involved with energy metabolism, oxidative stress, amino acid metabolism, and cell proliferation and differentiation were detected in ULF of pregnant HF than SF heifers; (5) The lipid content of the ULF is altered by pregnancy and fertility classification; (6) overall concentrations prostaglandins and interferon tau were increased in the uterine lumen of pregnant HF than SF heifers, likely due to differences in conceptus size. Collectively, results from these studies supports the idea that the dysregulated conceptusendometrium interactions in SF heifers affects the uterine luminal contents and impairs conceptus survival and elongation. Furthermore, knowledge gained from these studies enhances our understanding of the mechanisms regulating pregnancy loss in cattle and 
provides new information on uterine and conceptus biology during early pregnancy in ruminants. 


\section{SECTION I}

\section{LITERATURE REVIEW}

\section{Overview of pregnancy loss in cattle}

Subfertility is a prevailing problem in cattle and humans, and early pregnancy loss is a major cause of reduced reproductive efficiency in these species [1, 2]. Pregnancy success and embryonic mortality are affected by paternal, maternal, embryonic, and environmental factors [2-4], and the establishment and maintenance of pregnancy are a result of complex conceptus-endometrium interactions, that results in adequate conceptus (embryo/fetus and associated extraembryonic membranes) development, implantation and placentation $[3,4]$.

Understanding the timing of pregnancy loss is important to develop strategies to reduce such losses. Reliable pregnancy diagnosis in cattle can be currently performed as early as day 26 with the use of ultrasonography or by measuring placenta-derived pregnancy associated glycoproteins (PAGs) [5, 6]. However, accurate monitoring of preimplantation embryonic development from fertilization (day 0$)$ until implantation ( $\sim$ day 20 ) is still not possible. Historically, studies used to investigate early pregnancy events in cattle have been based on animal breeding/mating and subsequent animal slaughter or utilized surgical and non-surgical embryo collection approaches to estimate the incidences of fertilization failure and early embryonic mortality.

Fertilization rates have been estimated to be around $90 \%$ or higher in heifers, beef cows, and moderate-producing dairy cows [7-12], $\sim 80 \%$ in high-yielding dairy cows under thermoneutral conditions, and $\sim 60 \%$ in high-producing dairy cattle under heat stress $[2$, 13-15] . Thus, in thermoneutral conditions, the majority of the pregnancy losses occurs 
after fertilization, which encompasses the period of blastocyst formation, conceptus elongation, maternal recognition of pregnancy, implantation and placentation.

Fertilization is the fusion of the male and female gametes, and thus is influenced by maternal and paternal factors $[16,17]$. The maternal factors associated with reduced fertilization rates includes ovulation failure, oviduct obstruction [18], uterine inflammation $[14,19]$, high circulating progesterone concentrations near breeding $[2,20]$, and reduced oocyte quality $[12,15,21,22]$. Paternal factors implicated with reduced fertilization rates includes low sperm concentration [23, 24], semen containing sperm with abnormal morphology [25, 26], use of sexed sorted semen [27, 28], and with the use of semen of low sire conception rate (SCR) [29].

Available information concerning embryonic mortality in heifers indicates that $\sim 75$ $\%$ of beef and dairy heifers have a viable embryo 1 week following breeding/mating (Figure 1). Roche et al. [30] recovered 81\% (42/52) and 84\% (46/55) of viable embryos from beef heifers on days 3 and 8 following insemination. Sartori et al. [12] recovered $72 \%$ (23/32) of viable embryos from dairy heifers on day 6 days after breeding. Diskin et al. [7] recovered $77 \%$ (34/44) of viable embryos from beef heifers on day 8 following artificial insemination (AI). Maurer et al. [9] recovered 67\% (44/66) of viable embryos from beef heifers between days 2-6 post-mating. Embryonic mortality during the first week after fertilization has been associated with reduced oocyte quality (e.g. caused by heat stress [31], persistent follicles [11,32], low progesterone concentrations during ovulatory follicle development [33], and negative energy balance [17]), defects in embryonic genome activation [2], and influenced by dysregulated progesterone actions in the oviduct and uterus $[2,15]$. 
Studies investigating embryonic viability in the second week of gestation in heifers supports the idea that a low rate $(\sim 5 \%)$ of embryonic mortality occurs between the first and second week after fertilization. Roche et al. [30] reported that $75 \%(49 / 65)$ of inseminated beef heifers had a viable conceptus on day 14 after breeding. Diskin el al. [7] and Maurer et al. [9] recovered 59\% (23/39) and 70\% (21/30) of viable conceptuses from beef heifers on day 16 days after breeding/mating. Dunne et al. [34] and Christenson et al. [35] reported 68\% (32/47) and 64\% (30/47) of viable conceptuses been recovered from slaughtered dairy and beef heifers on days 14 and 12-15, respectively. Berg et al. [36] reported a higher rate of embryonic mortality ( $\sim 24 \%)$ between days 7 and 16 in heifers and nonlactating dairy cows upon transfer of multiple embryos produced in vitro (IVP). The increased embryonic mortality by day 16 observed in Berg's experiment may be attributed with the reduced capacity of IVP embryos to establish pregnancy as compared to embryos produced in vivo through AI or natural service.

More recent data have estimated pregnancy loss around time of maternal recognition of pregnancy, implantation, and placentation in heifers. Ortega et al. [29] estimated pregnancy status in dairy heifers on day 19 following AI based on the expression of interferon stimulated genes (ISGs) by white blood cells (WBC), and an overall pregnancy loss of $14.8 \%$ was suggested to have occurred between days 19 and 33 among heifers bred with high and low SCR semen. Additionally, Reese et al. observed that $14 \%$ of dairy heifers $(8 / 57)$ that were diagnosed nonpregnant on day 31 had increased PAG concentrations on day 24 , indicating that embryonic mortality in these animals occurred after the beginning of placental development [37].

The rate of embryonic mortality in heifers is greatly reduced after the first month of gestation, as only about $5 \%$ of fetal losses are estimated to occur between days 30 and 
90 in beef and dairy heifers [38, 39], and the rate of pregnancy loss significantly reduces as pregnancy advances past day 60 (Figure 1).

Pregnancy failure in lactating dairy cows are generally higher than in nonlactating cows and heifers, and a possible antagonism between high milk production and reproductive performance has long been suggested [17, 39-41]. High producing dairy cows experience a state of negative energy balance (NEB) during early lactation, as the nutrient demands required for supporting increasing milk production exceeds the dietary intake [42]. Negative energy balance is deleterious to fertility [43], and cows experiencing NEB are at higher risk of developing postpartum diseases [44-46]. Uterine postpartum diseases (e.g. retained fetal membranes, metritis, endometritis) are particularly detrimental to fertility, and higher rates of pregnancy loss are observed in animals that developed such conditions [14].

Pregnancy loss in lactating dairy cows during the first week after breeding have been recently reviewed [2], and is summarized in Table 1 . The reported losses are related to the first 6-7 days following $\mathrm{AI}$ in estrus or timed-AI (TAI), and thus includes fertilization failure and embryonic mortality. Pregnancy failure in the first week averaged $49 \%$ under thermoneutral conditions and $72 \%$ under heat stress (Table 1) [13].

Heat stress greatly increases pregnancy failure in the first week following AI, which is at least in part associated with the negative effects of heat shock on oocyte quality and early embryonic development [31]. Heat stress negatively affects ovarian follicle development [47, 48], impairs oocyte maturation [49, 50], oocyte fertilization [12], and reduces blastocyst rate due to oocyte damage [21, 22, 51]. Besides reducing oocyte quality, 1-cell and 2-cell embryos are very susceptible to heat shock [52-54], but at the morula (16cell, $\sim$ day 4) stage, embryos are significantly more resistant to heat stress [52-55]. The 
increased resistance of 16-cell embryos to the increase in body temperature confers embryo transfer (ET) as a viable alternative to AI during the summer months, as ET bypasses some of the negative effects of heat shock on oocyte quality and early embryo development [ 31 , $56]$.

Embryonic losses during time of maternal recognition of pregnancy, implantation and placentation in lactating cows have also been estimated. Reese et al. [37] reported that $\sim 14 \%$ (22/162) of lactating dairy cows diagnosed nonpregnant on day 31 after breeding had increased PAG concentrations on day 24. Additionally, Wijma et al. [57] estimated that $35 \%(14 / 40)$ of lactating cows diagnosed nonpregnant at day 42 had experienced early embryonic mortality, which $50 \%$ (7/14) of the losses were estimated to occur before day 24 , and $50 \%$ (7/14) between days 24 and 42, based on combined data from ISG expression by WBC and circulating PAG concentrations.

The timing of pregnancy loss in high producing dairy cows from fertilization to term have been reviewed $[2,15]$, and up to $60 \%$ of embryonic/fetal mortality was estimated to occur from fertilization to term, with $75 \%$ of those losses occurring by day 28 . Pregnancy loss after the first month of gestation is increased in high-producing lactating dairy cows than in heifers, and $\sim 12 \%$ of fetal losses are estimated to occur between days 30 and 90 , and $\sim 4 \%$ between day 90 and calving in modern high-producing lactating dairy cows [15].

Collectively, the available data indicates that fertilization failure and early embryonic mortality during the first month of gestation are the major causes of reproductive wastage in heifers and lactating dairy cows. Hence, understanding the maternal factors mediating embryonic development and pregnancy establishment is highly desirable, in order to develop strategies to improve reproductive efficiency and profitability in the beef and dairy industries. 


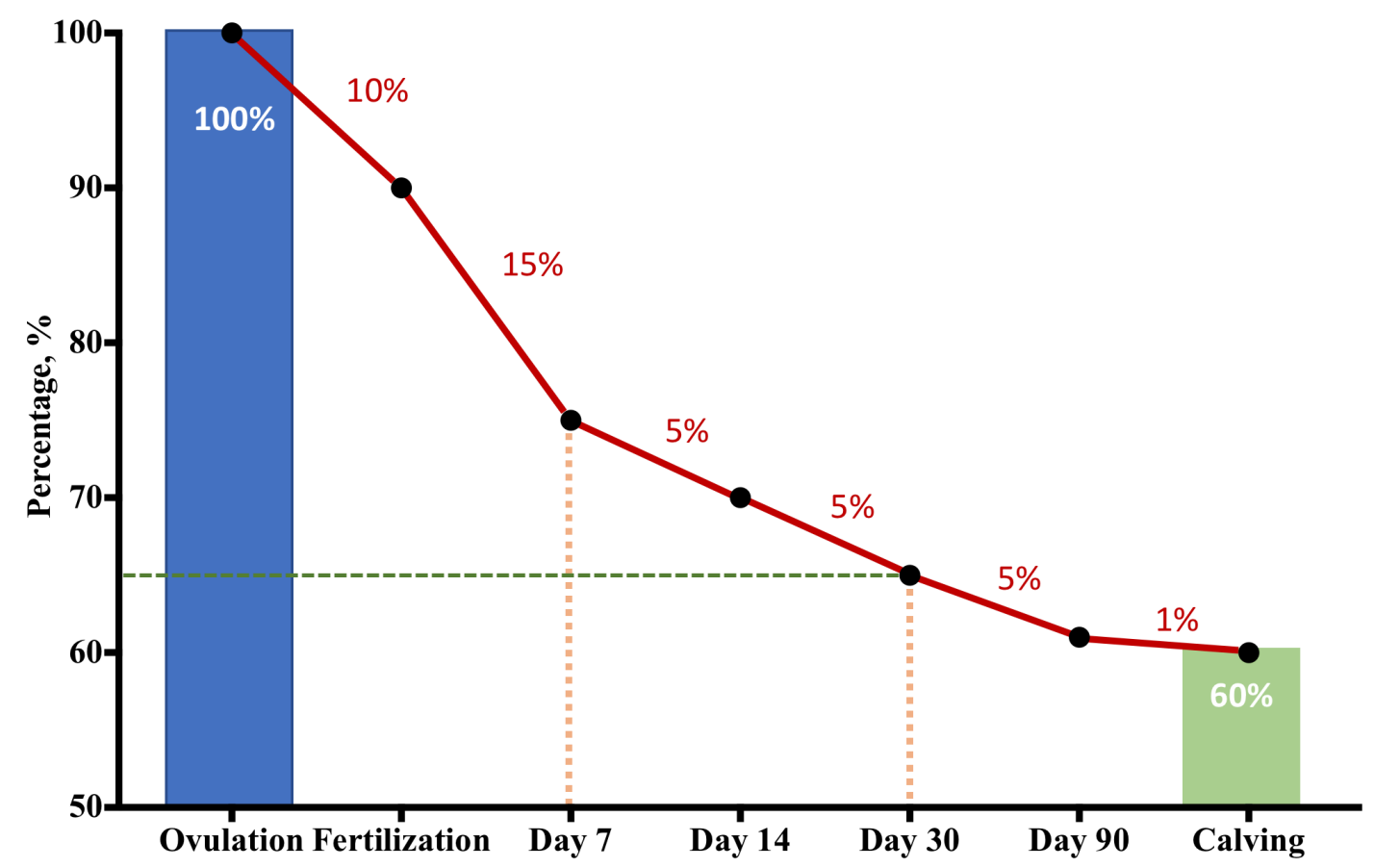

Figure 1. Proposed rate of pregnancy loss from ovulation through calving in beef heifers.

The data used to generate this graph suggests that fertilization failure and embryonic mortality accounts for $\sim 41 \%$ of pregnancy failure in heifers. Around $61 \%$ of the losses are estimated to occur within the first week following breeding, $\sim 12 \%$ after blastocyst formation and before maternal recognition of pregnancy, $\sim 12 \%$ during period of maternal recognition of pregnancy, implantation and beginning of placentation, $\sim 12 \%$ between days $30-90$ of gestation, and $\sim 2.4 \%$ from day 90 to term. 
Table 1. Pregnancy loss in lactating dairy cows during the first week following artificial insemination.

The data is presented as percentage of viable embryos (VE) per total structures (embryos or oocytes; OE) collected (VE/VO), and per total embryos collected (VE/TE) (Table adapted from [13]).

\begin{tabular}{|c|c|c|c|c|c|c|}
\hline \multirow[t]{3}{*}{ Reference } & \multicolumn{4}{|c|}{ AI after estrus } & \multirow{2}{*}{\multicolumn{2}{|c|}{$\begin{array}{l}\text { TAI } \\
\text { Thermoneutral }\end{array}$}} \\
\hline & \multicolumn{2}{|c|}{ Heat stress } & \multicolumn{2}{|l|}{ Thermoneutral } & & \\
\hline & $\mathrm{VE} / \mathrm{OE}$ & $\mathrm{VE} / \mathrm{TE}$ & $\mathrm{VE} / \mathrm{OE}$ & $\mathrm{VE} / \mathrm{TE}$ & $\mathrm{VE} / \mathrm{OE}$ & $\mathrm{VE} / \mathrm{TE}$ \\
\hline Wiebold (1988)a [58] & & & $48.0(12 / 25)$ & $48.0(12 / 25)$ & & \\
\hline Ryan et al. $(1993)^{\mathrm{b}}$ [59] & $47.1(24 / 51)$ & $58.5(24 / 41)$ & $41.0(16 / 39)$ & $51.6(16 / 31)$ & & \\
\hline Sartori et al. $(2002)^{\mathfrak{c}}[12]$ & $18.4(7 / 38)$ & $33.3(7 / 21)$ & $46.3(19 / 41)$ & $52.8(19 / 36)$ & & \\
\hline Cerri et al. (2009)e [61] & & & $61.0(25 / 41)$ & $71.4(25 / 35)$ & $59.8(76 / 127)$ & $69.1(76 / 110)$ \\
\hline Cerri et al. $(2009)^{\mathrm{f}}[62]$ & & & & & $51.8(44 / 85)$ & $65.7(44 / 67)$ \\
\hline Hackbart et al. (2010)a [63] & $20.0(4 / 20)$ & $57.1(4 / 7)$ & $42.2(27 / 64)$ & $50.0(27 / 54)$ & & \\
\hline \multicolumn{7}{|c|}{$\begin{array}{l}\text { 7-day-old embryos. } \\
\text { b 6- or 7-day-old embryos. } \\
\text { 6-day-old embryos. } \\
\text { d 5-day-old embryos of cows supplemented with calcium salt of palm oil or calcium salt of linoleic and trans-octadecenoic } \\
\text { acids. } \\
\text { e 6-day-old embryos. }\end{array}$} \\
\hline
\end{tabular}




\section{Early pregnancy development in ruminants}

\section{Uterus}

Major components of the female reproductive tract include the uterus, ovaries, Fallopian tubes (oviducts), cervix, vagina and external genitalia [64]. The uterus is the focus of this section, and its main functions include sperm transport and capacitation [65], control of estrus cycle [66], histotroph secretion [67], and maternal contribution to the placenta [68] and parturition [69].

During embryonic development, the uterus originates from the Mullerian ducts [70], and is still not fully differentiated at birth in domestic animals and humans. The main morphogenetic events that occurs postnatally includes the development of uterine glands (adenogenesis), organization and stratification of the endometrial stroma, and the differentiation and growth of the myometrium [71, 72].

The mammalian uterine wall is composed by three distinct layers, the endometrium, the myometrium and the perimetrium (Figure 2). The endometrium is the inner layer of the uterus consisting of luminal (LE) and glandular epithelium (GE), as well as underlying stroma. The myometrium is composed by an inner circular and an outer longitudinal layer of smooth muscle. The perimetrium is the serosal layer of the uterus, and corresponds to the part of the peritoneum that surrounds the uterus, and is composed of a membrane of simple squamous epithelium on top of a thin layer of loose connective tissue $[70,71]$.

The endometrium of an adult cow or ewe contains caruncular and intercaruncular areas. The caruncular endometrium is formed from proliferation of the subepithelial connective tissues, and plays important role on superficial implantation and placentation in ruminants. The synepitheliochorial placenta is formed by uterine caruncles fusing with 
placental cotyledons to form the placentomes, which are responsible for maternal-fetal exchanges (e.g. gaseous, nutrients and metabolic waste) [73]. The caruncular endometrium lacks uterine glands, but a profuse amount of glands are present in the intercaruncular endometrium of cows and sheep [71].

Uterine gland development involves budding of GE from the LE [74], and although the precise mechanism regulating the differentiation of LE cells into GE cells have not been elucidated, uterine adenogenesis is regulated by complex intrinsic (e.g. transcription factors, growth factors) and extrinsic stimulus (e.g. ovary, pituitary, mammary gland) [75]. Gland secreted factors plays important role on preimplantation embryonic development in sheep and mice, as conceptus elongation does not occur in uterine gland knockout sheep (UGKO) [76, 77], and glandless mice display implantation and decidualization issues [78, 79]. Uterine luminal constituents, collectively known as histotroph, is composed of molecules secreted or transported into the uterine lumen [80]. The histotroph from cattle has been characterized [81-85], and is a complex mixture of amino acids, glucose, lipids, proteins, vitamins, ions, cytokines, hormones, growth factors, among other substances. Because the attachment of the bovine conceptus with the endometrial luminal epithelium occurs around day 20 [86], and microvillar interdigitation between trophoblast cells and the caruncular epithelium which marks the beginning of placentation only begins around day 24 [87], the development of preimplantation embryo/conceptus prior attachment and placentation is highly depended on histotrophic nutrition. 


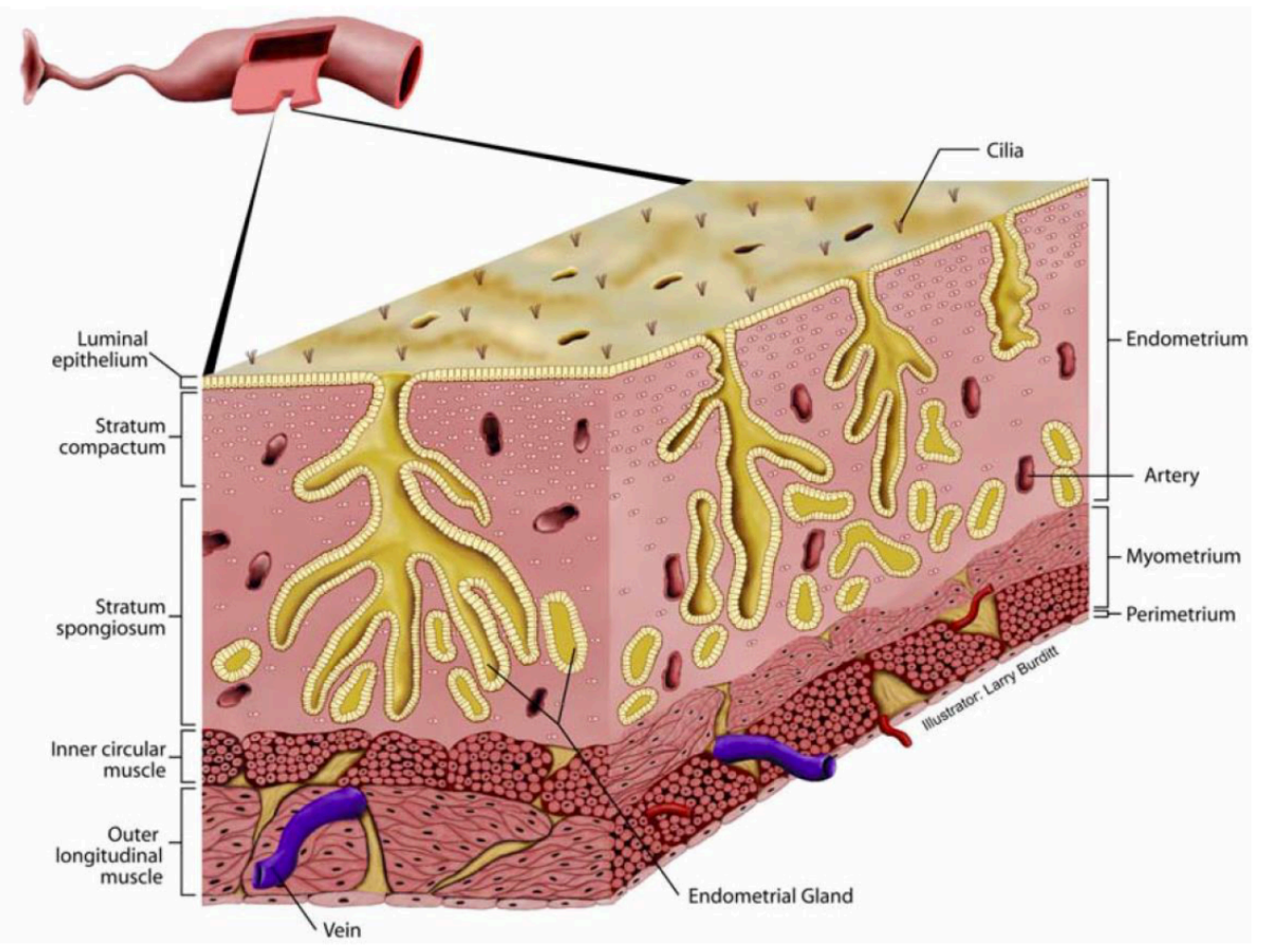

Figure 2. Uterine histoarchitecture.

The uterine wall is composed by three distinct layers, the endometrium, the myometrium and the perimetrium. The endometrium is the inner layer, and is consisted of luminal epithelium (LE), glandular epithelium (GE), and stroma. The myometrium is composed by an inner circular and outer longitudinal layer of smooth muscle. The perimetrium is the serosal layer of the uterus, which is consisted of superficial mesothelium and lose connective tissue. (The figure is courtesy of Rodney Geisert and Larry Burdett, Oklahoma State University, Stillwater, USA) 


\section{Preimplantation embryonic development}

During mammalian embryonic development, the oocyte is fertilized in the ampulla of the oviduct forming a zygote [88], which undergo several mitotic divisions to form a morula [89] that enters the uterus between days 4 to 6 post-mating in cattle [90]. The morula then develops into a blastocyst that contains an inner cell mass (ICM) and a blastocoele or central cavity surrounded by a monolayer of trophectoderm (TE) cells [91]. The cells from the ICM are pluripotent and form all three embryonic germ layers (endoderm, mesoderm and ectoderm), and the differentiation of TE cells gives rise to the extraembryonic structures, including the placenta [92].

The bovine blastocyst is a 150 to $190 \mu \mathrm{m}$ sphere on day 7 [93], and undergoes tremendous morphological changes (Figure 3) in the uterus including hatching from the zona pellucida (days 9-10), transitioning from its initial spherical appearance, into an ovoid (0.5-4mm), tubular $(5-19 \mathrm{~mm})$, and then filamentous $(20-60 \mathrm{~mm})$ form by day 15 of pregnancy $[36,94,95]$. The process of conceptus elongation is uterine dependent, as hatched blastocysts and trophoblastic vesicles do not elongate in vitro, but elongation is possible upon their transfer into the uterus of cows and sheep [96, 97].

Adequate conceptus development is critical for successful maternal recognition of pregnancy and implantation [98]. According to Guillomot [99], the implantation process in domestic ruminants includes 5 phases: (1) blastocyst shedding of the zona pellucida; (2) pre-contact and blastocyst orientation; (3) apposition; (4) adhesion; and (5) endometrial invasion (Figure 4). Apposition of the conceptus TE and endometrial LE begins around day 16 in cattle, attachment is achieved around day 20 , and the microvillar interdigitation between trophoblast cells and the caruncular epithelium is observed around day 24 [87]. 
During attachment of mononuclear TE cells with endometrium LE ( day 20$)$, trophoblast giant binucleate cells (BNC) begin to differentiate within the TE [86], through successive nuclear divisions without cytokinesis $[100,101]$. The recently formed BNC then undergoes differentiation, in a process marked by migration of BNC away from the basement layer and tight junctions of the trophoblast. Their nuclei becomes polypoid, and their Golgi apparatus start to produce a large number of granules containing pregnancy associated glycoproteins (PAGs) and placental lactogen (also known as chorionic somatomammotropin hormone 1 or $\mathrm{CSH} 1$ ). Once fully granulated, $\mathrm{BNC}$ move towards the maternal side, and fuse with endometrial LE cells, thereby forming the trinucleated fetomaternal hybrid cell [102]. In sheep, the continued fusion of BNC and trinucleated cells results in the formation of syncytial plaques, that contains $20-25$ nuclei, and are linked by tight junctions $[100,101]$. The formation of syncytial plaques results in expansion of fetal villi and fetomaternal interface, and participates in the synthesis and delivery of placental secreted factors [101] (Figure 4). In cattle, however, areas of syncytium appears only to be formed at time of implantation [86]. Although the function of PAGs remains unknown, CSH1 binds to prolactin receptors expressed exclusively by the endometrial GE, stimulating uterine gland morphogenesis and function [103], thus affecting histotroph composition and conceptus development during early pregnancy.

Histotrophic nutrition is particularly important prior to placentome development in ruminants. In cattle, the allantois begins to form around day 23 , and its vascularization is well visible on day 26 . The vascularization of the allantois is a critical step in placental development, as vessels from the allantois vascularize the chorion, and cotyledons originates from the outside of the chorion ( $\sim$ day 30$)$. Around day 50, placental cotyledons fuses with uterine caruncles forming placentomes, which well established by day 70 of 
pregnancy [104]. Upon placentome formation, there is a switch between histotrophic to hematatrophic nutrition. Importantly, histotrophic nutrition of fetus continues during pregnancy, through specialized areas of the placenta named areolae [105]. 


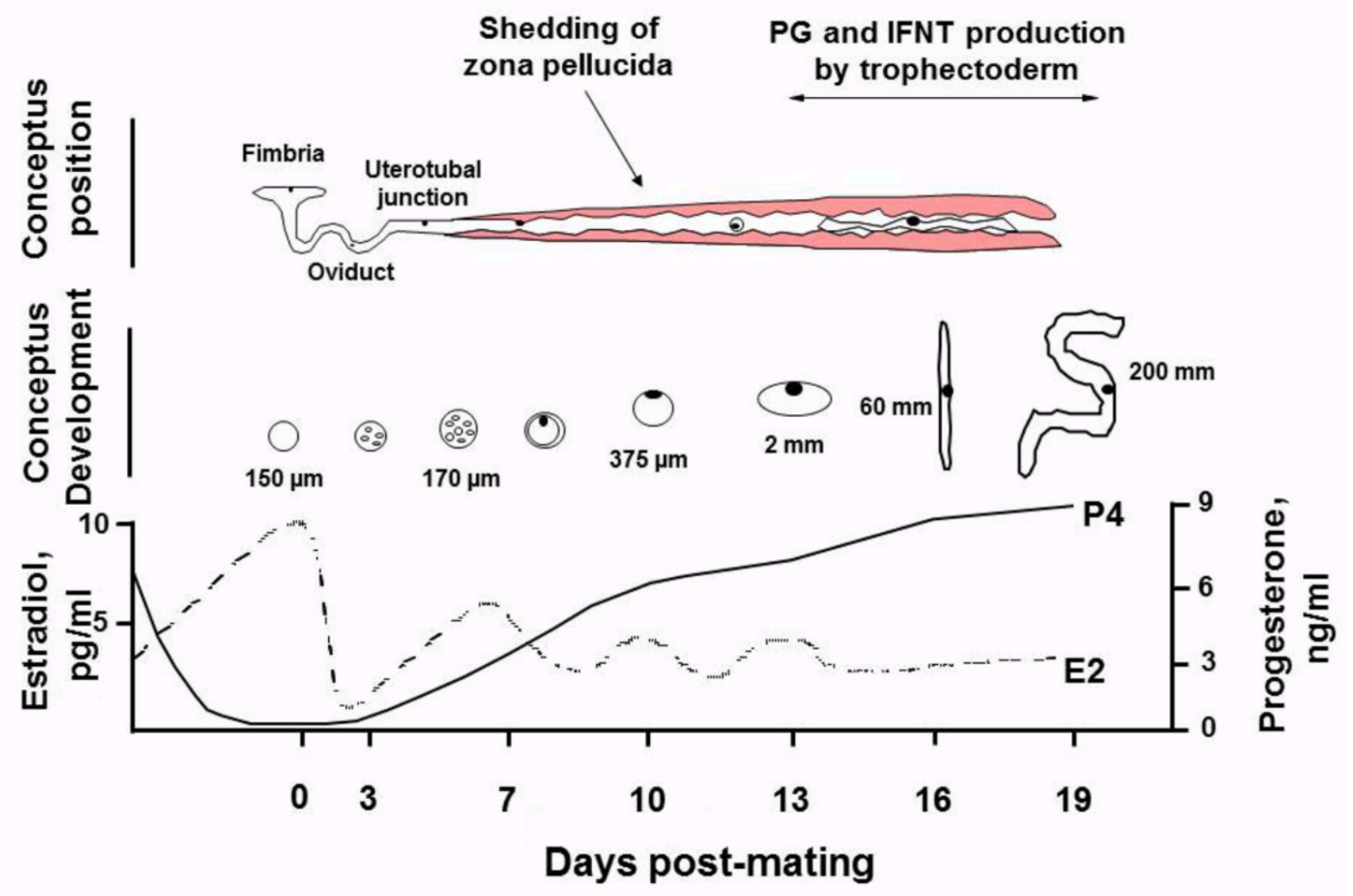

Figure 3. Early embryonic development in cattle from ovulation to attachment.

Summary of events occurring during the migration of oocytes and developing embryo/conceptus in the female reproductive tract, and the circulating concentrations of estradiol (E2) and progesterone (P4) in the maternal blood during this period. The oocyte is fertilized in the ampulla of the oviduct forming a zygote, and a morula enters the uterus between days 4 to 6 post-mating. The blastocyst is formed on day 7, and it hatches from the zona pellucida around days 9-10. At day 12 the conceptus is ovoid, and it becomes tubular by days 13-14, and mostly filamentous at or after day 15 . The elongating conceptus secretes interferon tau (IFNT) and prostaglandins (PG), which are essential for maternal recognition of pregnancy and to modulate uterine gene expression for successful implantation and placentation. Figure from [106] 


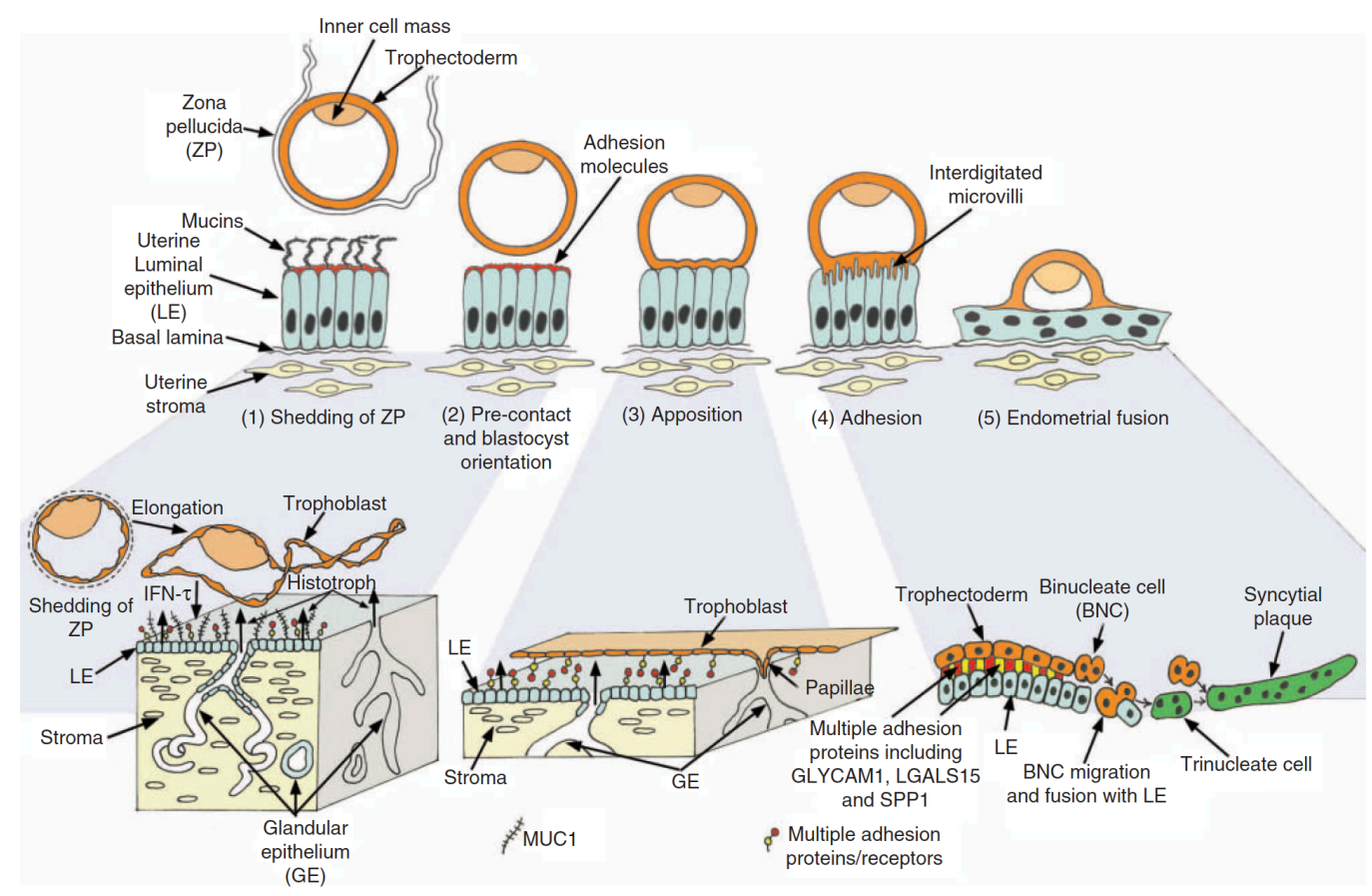

Figure 4. Phases of implantation in sheep.

The bovine blastocyst hatches from the zona pellucida around days 9-10, exposing the trophectoderm (TE) to be in direct contact with the uterine luminal epithelium (LE) (Phase 1). However, at this stage, anti-adhesive mucin 1 (MUC1) are present on the surface of the endometrium LE, limiting the contact of conceptus TE and adhesive receptors (e.g. integrins) expressed by the LE. Thus, during this period, the developing conceptus rely heavily in histotrophic nutrition. Phase 2 consists of pre-contact and blastocyst orientation. Apposition of the conceptus TE and endometrial LE begins around day 16 (Phase 3). At this stage, expression of MUC1 by the endometrium LE declines, exposing LE integrins which facilitates conceptus attachment. Attachment of conceptus TE and endometrium LE is achieved around day 20, and the microvillar interdigitation observed around day 24 (Phase 4). Several uterine secreted molecules, such as glycosylated cell adhesion molecule (GLYCAM1) 1, galectin 15 (LGALS15) and secreted phosphoprotein 1 (SPP1 or osteopontin), interact with receptors (integrins and glycoconjugates) on the apical surface 
of trophectoderm and LE to facilitate adhesion. Phase 5 involves the fusion of trophoblast giant binucleate cells (BNC) and uterine LE forming the trinucleated cells. The continued fusion of $\mathrm{BNC}$ and trinucleated cells results in the formation of syncytial plaques. Figure from [98]. 


\section{Maternal recognition of pregnancy}

The regulatory mechanisms controlling the estrus cycle and pregnancy recognition are substantially different among mammalian species. Primates for instance, have uterine independent menstrual cycle, as the luteolytic prostaglandin F2 $\alpha$ (PGF2 $\alpha)$ is derived from intra-ovarian sources, and conceptus-derived chorionic gonadotropin (CG) plays a key role ensuring corpus luteum (CL) maintenance and sustained progesterone production [107]. Ruminants on the other hand, have uterine dependent estrus cycle, as the luteolytic pulses of PGF $2 \alpha$ are rather secreted by endometrium cells, and interferon tau (IFNT) is the major conceptus secreted factor responsible for maternal recognition of pregnancy [108].

IFNT is a type 1 interferon (IFN) produced exclusively by mononuclear trophectoderm cells of elongating conceptuses in ruminants, and in cattle it is secreted predominantly between days 15-24 [109-111]. IFNT prevents luteolysis by suppressing the expression of oxytocin receptor (OXTR) in the uterine luminal (LE) and superficial glandular epithelia (sGE), which abrogates the uterine luteolytic mechanism $[108,112$, 113] (Figure 5). All type 1 interferons, including IFNT, signals through a common receptor (IFNAR)[114, 115], which is a heterodimeric receptor composed of IFNAR1 and IFNAR2 subunits [116]. All endometrial cells express IFNAR, but IFNT regulation of endometrial gene expression occurs in a cell type-specific manner. Expression IRF2, a potent and stable transcription repressor, increases in the endometrial LE and sGE during early pregnancy. The presence of IRF2 inhibits the expression of classical interferon stimulated genes (ISGs) in the LE/sGE, an event mediated by the direct binding of IRF2 to IFN-stimulated response elements (ISRE) and IFN response factor binding element (IRFE) that are present in the promoter region of many classical ISG, thus suppressing gene transcription. However, IRF2 is not upregulated in the endometrial stromal (ST) or deep glandular epithelium 
(dGE) by pregnancy, which allows classical ISGs to be upregulated in these cell types upon IFNT signaling [117].

Differences exist in the mechanisms by which IFNT regulates luteolysis in cattle and sheep, as the bovine OXTR gene lacks a classical palindromic estrogen response element [118], and no changes in ESR1 expression are observed in uterine epithelia of pregnant and nonpregnant cows. Thus, in cattle, IFNT and pregnancy can suppress OTRX expression independently of ESR1. Nonetheless, IRF2 have also been shown to suppress OXTR expression in the bovine LE [118], indicating a common role of IRF2 mediating the antiluteolytic actions of IFNT in both, the bovine and ovine endometria [112].

IFNT signaling in the endometrium induces the expression of classical and nonclassical interferon stimulated genes (ISGs) [117], which are hypothesized to play essential role establishing uterine receptivity in ruminants $[98,117,119,120]$. The classical signaling of IFN occurs through the JAK/STAT (janus kinase/signal transduce and activator of transcription) pathway, and non-classical signaling cascades includes PI3K and p38 MAP kinase pathways [121].

A variety of IFNT genes exist in most ruminant species studied (Figure 2), and the transcriptional regulation of IFNT expression in these species appears to be somewhat conserved [122]. The conceptus expresses several transcription factors that have been implicated with the regulation of IFNT transcription. Transcription factor such as POU5F1 (also known as Oct-4) [123] and T-box protein eomesodermin (EOMES) [124] have been reported to suppress IFNT expression, while others such as ETS2 [125-127], distal-less 3 (DLX3) [128], CDX2 [124, 129] and GATA2/3 [130, 131] have been implicated with the upregulation of IFNT transcription. Additionally, a transcriptional co-activator expressed 
by ovine conceptus, the cAMP-response element-binding protein-binding protein (CREBBP), have been shown to upregulate of IFNT transcription [132] (Figures 7).

Uterine derived factors are also thought to mediate conceptus expression of IFNT (Figure 7). For instance, colony stimulated factor 1 (CSF1), interleukin 3 (IL3), and fibroblast growth factor-2 (FGF2) are expressed by the endometrium LE and GE in sheep, and have been shown to induce conceptus expression of IFNT in vitro [133-135]. 


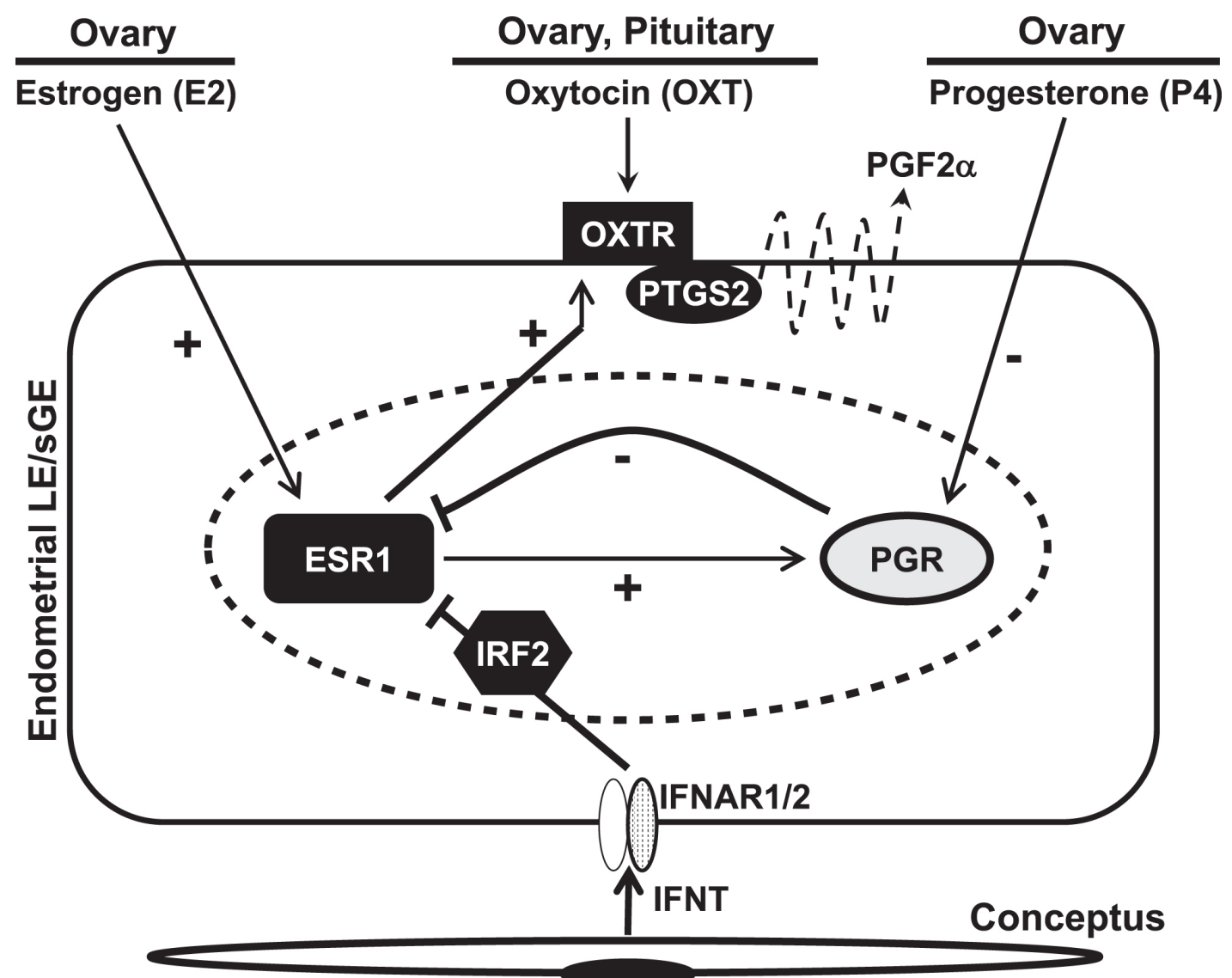

Figure 5. Regulation of the estrus cycle in pregnant and nonpregnant ewes.

During estrus and metestrus, estradiol produced by growing follicles stimulates the increase in expression of estrogen receptor alpha (ESR1) in the endometrial luminal (LE) and superficial glandular epithelium (sGE), which in turn increases the expression of progesterone receptor (PGR) and oxytocin receptor (OXTR) in these cells. Although PGR is expressed by endometrial $\mathrm{LE} / \mathrm{sGE}$ during estrus, ESR 1 is not downregulated by PGR signaling at this stage, as low progesterone concentrations are detected at estrus. Thus, ESR1 and OXTR are upregulated during estrus, but the luteolytic pulses of prostaglandin F2 $\alpha$ (PGF2 $\alpha$ ) does not occur at this stage due to the absence of corpus luteum (CL) derived oxytocin. The increase in systemic progesterone in early diestrum results in downregulation of ESR1 and OXTR expression via PGR signaling, resulting in low 
expression of ESR1 and OXTR during the first 8 to 10 days of the estrus cycle. Nonetheless, continuous exposure of endometrial LE/sGE cells to progesterone induces downregulation of PGR in these cell types around days 11 to 12 of the cycle. Consequently, with the absence of PGR signaling blocking ESR1 and OXTR expression, ESR1 becomes upregulated in LE/sGE cells around days 11 and 12, and upregulation of OXTR is observed on days 13 to 14 . Increasing estradiol produced by growing follicles during this period further stimulates ERS1 signaling. The release of oxytocin by the CL begins around day 9 . In nonpregnant ewes, oxytocin released by the CL and neurohypophysis bind to its receptor and induce the luteolytic pulses of PGF2 $\alpha$, resulting in CL regression and reinitiation of the estrus cycle. In pregnant ewes, interferon tau (IFNT) is secreted by the elongating conceptus and binds to its receptor (IFNAR) expressed by all endometrial cell types. IFNAR is a heterodimeric receptor composed of IFNAR1 and IFNAR2 subunits, and it's signaling induces the expression of IRF2 in the endometrial LE and sGE. IRF2 is a potent transcriptional repressor that inhibit the expression of classical interferon stimulated genes (ISGs), including ESR1. ESR1 transcriptional repression prevents OXTR upregulation and therefore inhibits the uterine luteolytic mechanism ewes. Figure from [108] 


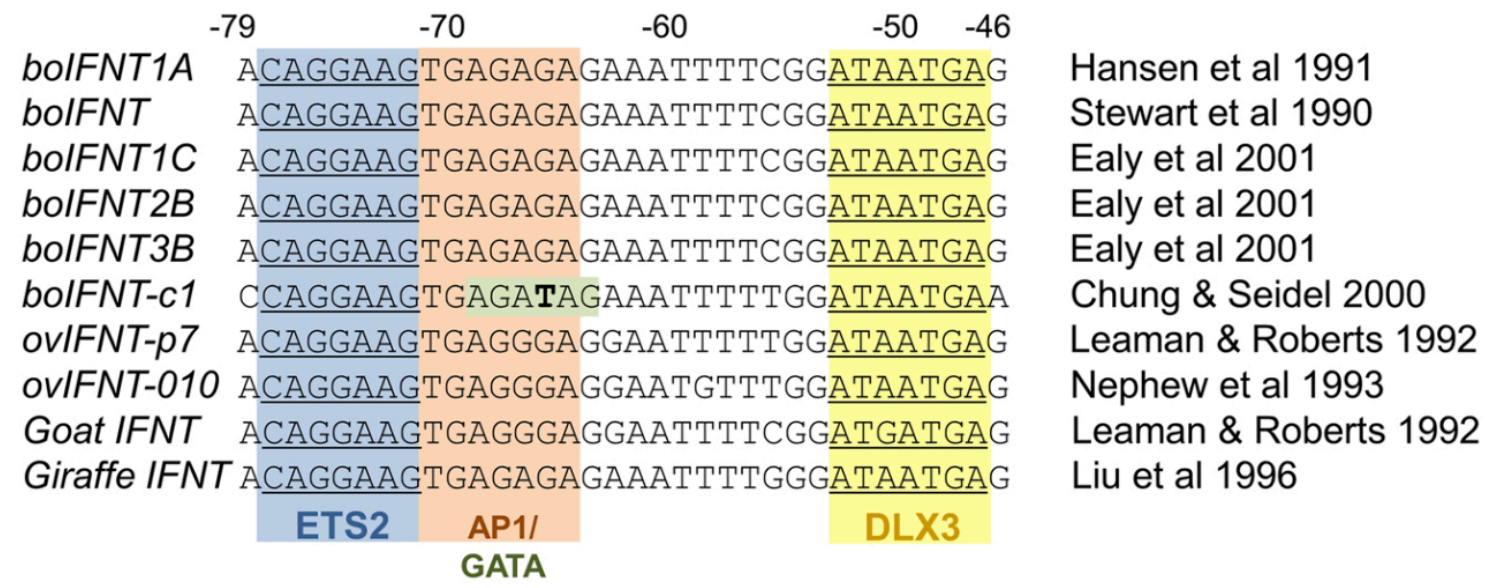

Figure 6. Conserved transcription factor biding sites on a variety of interferon tau (IFNT) genes in ruminants.

The transcriptional regulation of IFNT appears to be somewhat conserved across species, as different IFNT genes have been shown to contain a conserved ETS-2 binding site (blue), putative AP1-binding site (orange) or GATA-binding site (green), and DLX3-biding site (yellow). Figure adapted from [122] 

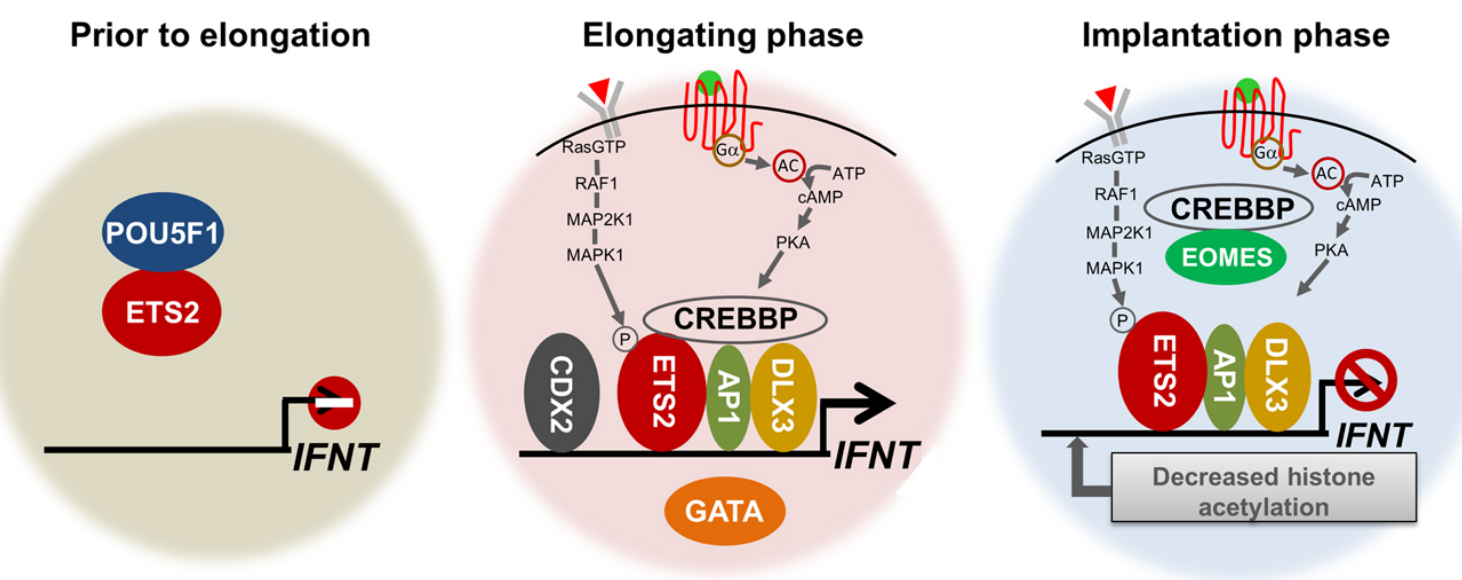

Figure 7. Suggested model of transcriptional control of interferon tau (IFNT) expression during preimplantation embryonic development.

Ezashi and Imakawa [122]. Prior to conceptus elongation, POU5F1 is thought to inhibit IFNT expression by squelching ETS2, an important IFNT transactivator. As conceptus development proceeds, the expression of POU5F1 is reduced, allowing ETS2 to increase IFNT transcription. Additionally, as the conceptus elongate, the expression of other IFNT transactivators (DLX3, CDX2, GATA2/3) increase in the trophectoderm, and growth factors released from the maternal endometrium are also thought to further stimulate IFNT transcription through the activation of MAPK and PKA signaling pathways. Conceptus expression of IFNT reduces around time of implantation, but the precise mechanisms mediating this event remains unclear. Trophectoderm expression of EOMES increases following conceptus attachment, and because EOMES has been reported to bind to CREBBP reducing CREBBP-mediated transcriptional co-activation of IFNT, it has been hypothesized to participate in the mechanism resulting in IFNT silencing during implantation. Epigenetic mechanisms are also thought to suppress IFNT transcription during the implantation phase, as reduction in histone acetylation in the upstream region of 
the IFNT gene has been observed in ovine conceptuses around time of implantation (day 20) as compared to preimplantation conceptuses (days 14 and 16). 


\section{Histotroph components}

\section{Glucose}

Glucose is a major energy source for mammalian cells, and its catabolism generates key substrates for protein and lipid biosynthesis [136], which are particularly important to support growth of rapidly proliferating cells such as cancer cells and preimplantation embryos [137-140].

Concentration of glucose in the uterine lumen have been reported to rise around time of conceptus elongation in sheep [139] and cattle [141], and three possible mechanisms may regulate uterine luminal concentrations of glucose in ruminants: 1) de novo synthesis of glucose (gluconeogenesis) by uterine cells; 2) glucose transport from blood into the uterine lumen by glucose transporters; or 3) combination of both, uterine gluconeogenesis and glucose transport.

Gluconeogenesis does not appear to occur in the uterus of mice and humans, because their uteri lacks expression of rate-limiting gluconeogenic enzymes [142, 143]. For instance, phosphoenolpyruvate carboxykinase (PEPCK, also known as PCK), which converts oxaloacetate into phosphoenolpyruvate and carbon dioxide, was not detected in mouse uterus [144], and PCK and fructose-1,6-bisphosphatase (FBP1), which converts fructose-1,6-bisphosphate to fructose 6-phosphate, were also not detected in human myometrium and endometrium [145]. However, Moore et al. [146] observed increased mRNA levels of gluconeogenic enzymes (PCK2, FBP1 and G6PC3) in bovine caruncular endometrium from days 28 to 42 of gestation, indicating that gluconeogenesis may occur in the bovine uterus during pregnancy. Although the gluconeogenic activity of the uterus remains poorly investigated in ruminants, the expression of glucose transporters by endometrial cells are thought to play important role modulating uterine luminal 
concentrations of glucose [138]. Because glucose is hydrophilic, it cannot diffuse through the lipid bilayer of plasma membranes, and thus the expression of carrier proteins by uterine cells are necessary to allow glucose uptake into the uterine lumen $[147,148]$. Mammalian cells express three families of glucose transporters, the facilitative glucose transporter family (solute carriers SLC2A/GLUT), the $\mathrm{Na}^{+} /$glucose cotransporter family (solute carriers SLC5A/ SGLT) [136], and the recently discovered SWEET (SLC50) sugar transporter $[149,150]$. Member of the glucose transporter families GLUTs and SWEET are classified as uniporters, which facilitates sugar transport based on sugar gradient. Members of the SGLT (SLC5A) family are classified as symporters, as they mediate the simultaneous transport of glucose and sodium (Figure 8). Several members of GLUT and SGLT families have been identified in uterus of human, mice [151], sheep [138], and cattle [152], and the expression of selected glucose transporters (SLC2A1, SLC5A1 and SLC5A11) have been shown to increase in pregnant compared to cyclic endometrium in sheep [138].

Glucose is an essential nutrient during embryonic development, as glucose metabolism provides energy and anabolic precursors that supports cellular replication and specialization [153], and may be of particular importance during the rapid proliferation of trophectoderm cells during conceptus elongation in ruminants. Most somatic cells, in the presence of oxygen, preferentially oxidize glucose through oxidative phosphorylation, as this pathway favors ATP production [154]. Oxygen serves as electron acceptor in the electron transport chain (ETC), and therefore is required for oxidative phosphorylation [155]. Thus, under anaerobic conditions, most differentiated cells diverge pyruvate away from the TCA cycle and converts it into lactate, a reaction known as anaerobic glycolysis [154]. Otto Warburg made the important observation that cancer cells, even under aerobic 
conditions, metabolize glucose preferentially into lactate, a phenomenon that became known as aerobic glycolysis or Warburg effect $[156,157]$. Similar to cancer cells, embryonic cells are highly proliferative, and thus the Warburg effect has been hypothesized to occur during the growth of preimplantation embryos [140].

Oxidative phosphorylation is a more efficient pathway for generating ATP from glucose than aerobic glycolysis, as 1 molecule of glucose generates up to 36 molecules of ATP through oxidative phosphorylation but only 2 molecules of ATP via aerobic glycolysis. Thus, even though aerobic glycolysis is an inefficient way to generate ATP, it is still the preferred pathway for the metabolism of glucose in most cancer cells. The main explanation for the switch from oxidative phosphorylation in normal somatic cells to aerobic glycolysis in cancer cells, is that highly proliferative cells have other critical metabolic requirements that extend beyond energy production. For instance, cells undergoing mitosis have large requirements for biomass production, as one parent cell has to duplicate all its contents to form two identical daughter cells, and this alternative metabolism (Warburg effect) diverges glucose to create macromolecular precursors for synthesis of fatty acids, amino acids, and nucleotides (Figures 9 and 10) [140]. For instance, oxidation of glucose in the pentose phosphate pathway (PPP) generates nicotinamide adenine dinucleotide phosphate (NADPH) and pentoses (Figure 9), such as ribose-5-phosphate (R5P) and erythrose 4-phosphate (E4P), which are important for the synthesis of nucleotide, nucleic acids and aromatic amino acids (e.g. phenylalanine, tyrosine, tryptophan, and histidine). Additionally, de novo biosynthesis of serine and glycine, which plays important role in biomass production, occur through a glycolysisdiverting pathway, as the glycolytic intermediate 3-phosphoglycerate can be converted to serine, and serine can be converted into glycine (Figure 9). 
The amino acid glutamine plays a key role in cells undergoing the Warburg effect, as glutamine metabolism provides an important carbon source for the synthesis of lactate from pyruvate [158]. Although glutamine is a nonessential amino acid that can be synthesized from glucose, it is a key substrate for highly proliferative cells, and some cancer cells lines have been described as 'glutamine addicted' because they cannot survive without exogenous glutamine $[159,160]$. Glutamine is also involved with energy production, and with the synthesis of nucleic acids, amino acids, and lipids (Figure 10). Additionally, glutamine participates in the biosynthesis of glutathione (GSH), a major antioxidant in mammalian cells [161] (Figure 11). Glutathione is a tripeptide consisted of glutamine-derived glutamate, glycine and cysteine. Glutamate-cysteine ligase (GCL), a heterodimer composted of a modifier (GCLM) and a catalytic (GCLC) subunit, catalyzes the ligation of glutamate and cysteine to form $\gamma$-glutamylcysteine, the first step in GSH synthesis. The second step in GSH synthesis is the coupling of $\gamma$-glutamylcysteine and glycine, a reaction catalyzed by glutathione synthetase (GSS) [162]. The antioxidant effects of GSH have been associated with the actions of glutathione peroxidase (GPX) and peroxiredoxin 6 ( $\mathrm{Prdx} 6)$, which can convert hydrogen peroxide $\left(\mathrm{H}_{2} \mathrm{O}_{2}\right)$ to water $\left(\mathrm{H}_{2} \mathrm{O}\right)$, forming glutathione disulfide (GSSG), the oxidized form of GSH [161, 163]. GSSG can be converted back to GSH, in a reaction catalyzed by glutathione reductase (GSR), through the conversion of NADPH to NADP+ [163] (Figure 11). The ratio of reduced (GSH) and oxidized (GSSG) glutathione (GSH/GSSG) indicates the magnitude of oxidative stress, and while a resting cell may have a GSH/GSSG ratio that exceeds 100:1, cells experiencing oxidative stress have been shown to possess lower GSH/GSSG ratio, of 10:1 or even 1:1 $[164,165]$ 
Glutamine metabolism is also thought to play a major role stimulating trophectoderm cell proliferation during conceptus elongation, as glutamine and glutamine derivatives synergize with other amino acids to regulate the activity of the mechanistic target of rapamycin complex 1 (mTORC1), a master regulator of cellular growth and proliferation $[163,166]$. For instance, $\alpha$-ketoglutarate and leucine have been shown to promote mTORC1 activation through different mechanisms (Figure 12). 


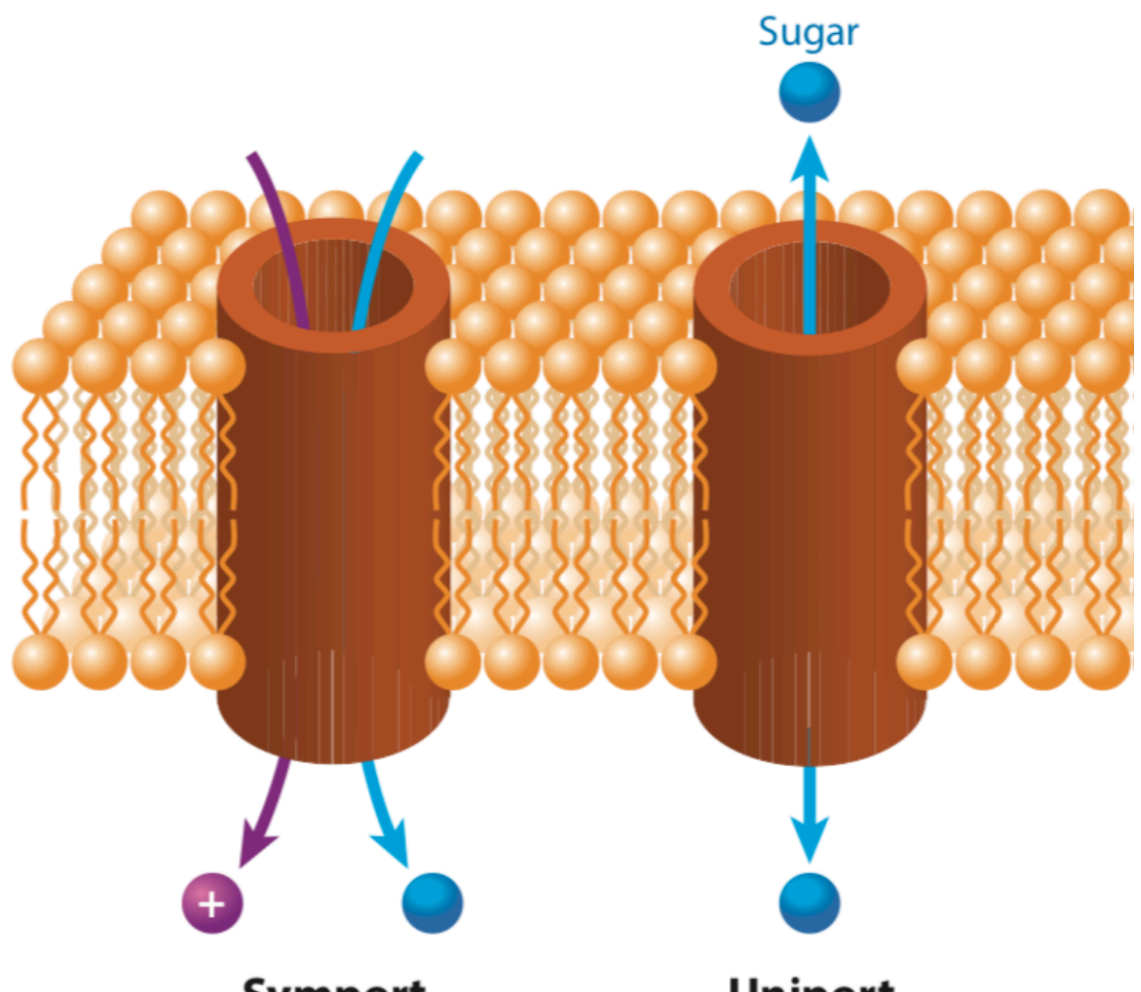

Symport

Uniport

Figure 8. Transport of glucose through glucose transporters.

The glucose transporter families GLUTs (SLC2A) and SWEET (SLC50) are classified as uniporters, which facilitates sugar transport based on sugar gradient. Members of the SGLT (SLC5A) glucose transporter family are classified as symporters, as they mediate the simultaneous transport of glucose and sodium. Figure adapted from [167]. 


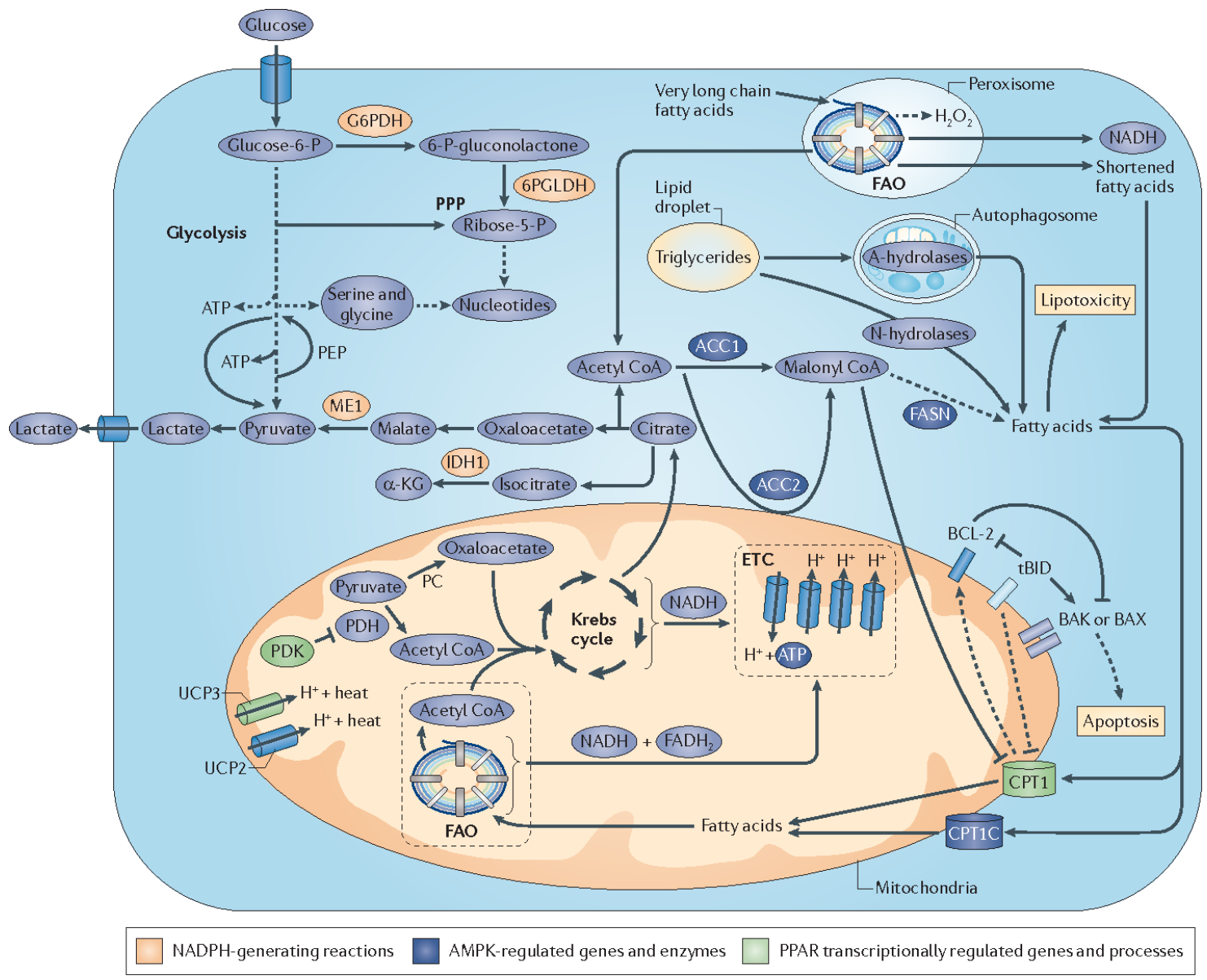

Figure 9. Glucose and fatty acid metabolism in cancer cells.

Glucose metabolism in cancer cells appears to be similar to that of in preimplantation embryos. The metabolic pathways represented include fatty acid metabolism, aerobic glycolysis, oxidative phosphorylation, serine and glycine biosynthesis, and pentose phosphate pathways (PPP). Figure from [168] 


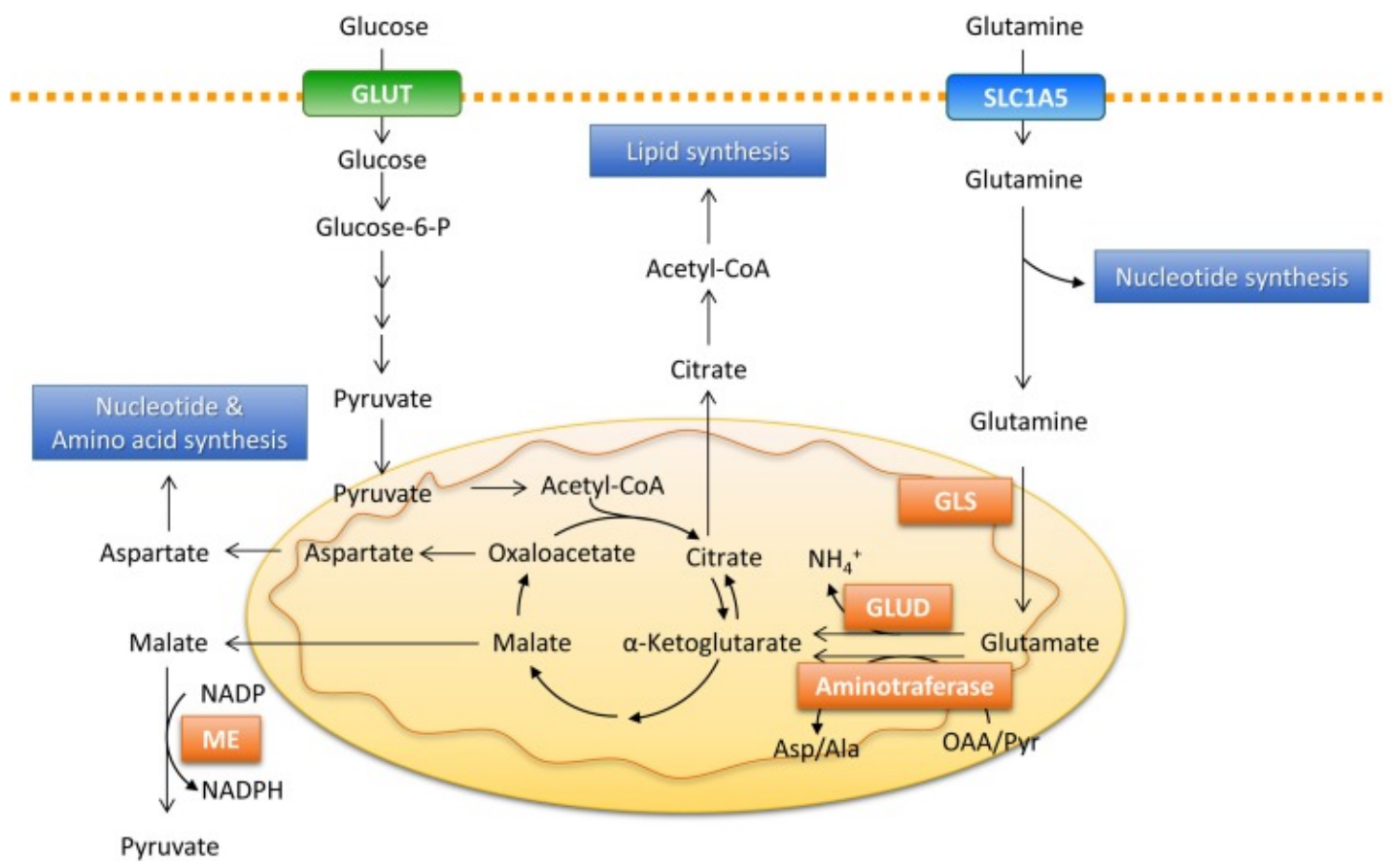

Figure 10. Major metabolic pathways involved with glutamine metabolism.

Glutamine uptake by mammalian cells occurs via a sodium-dependent neutral amino acid transporter (SLC1A5). After its uptake, glutamine can be directed for the synthesis of nucleotides, or be converted to glutamate by mitochondrial glutaminase (GLS). Aminotransferases catalyzes the redistribution of nitrogen between amino acids, and can convert glutamate to $\alpha$-ketoglutarate, producing alanine and aspartate. Glutamate can also be converted in $\alpha$-ketoglutarate by glutamate dehydrogenase (GLUD), and $\alpha$-ketoglutarate enters the TCA cycle to generate energy for the cell. In the TCA cycle, $\alpha$-ketoglutarate can be converted back into citrate, which can be exported from the mitochondria to the cytoplasm to be metabolized in acetyl-Coa and oxaloacetate, and acetyl-Coa is a key precursor for lipid synthesis. In the TCA cycle, $\alpha$-ketoglutarate is converted to malate, which can exit the mitochondria and produce pyruvate and NADPH through the actions of the malic enzyme (ME). Malate in the TCA cycle is converted to oxaloacetate, which can be converted to aspartate for supporting nucleotide and amino acid synthesis. Figure from [163] 


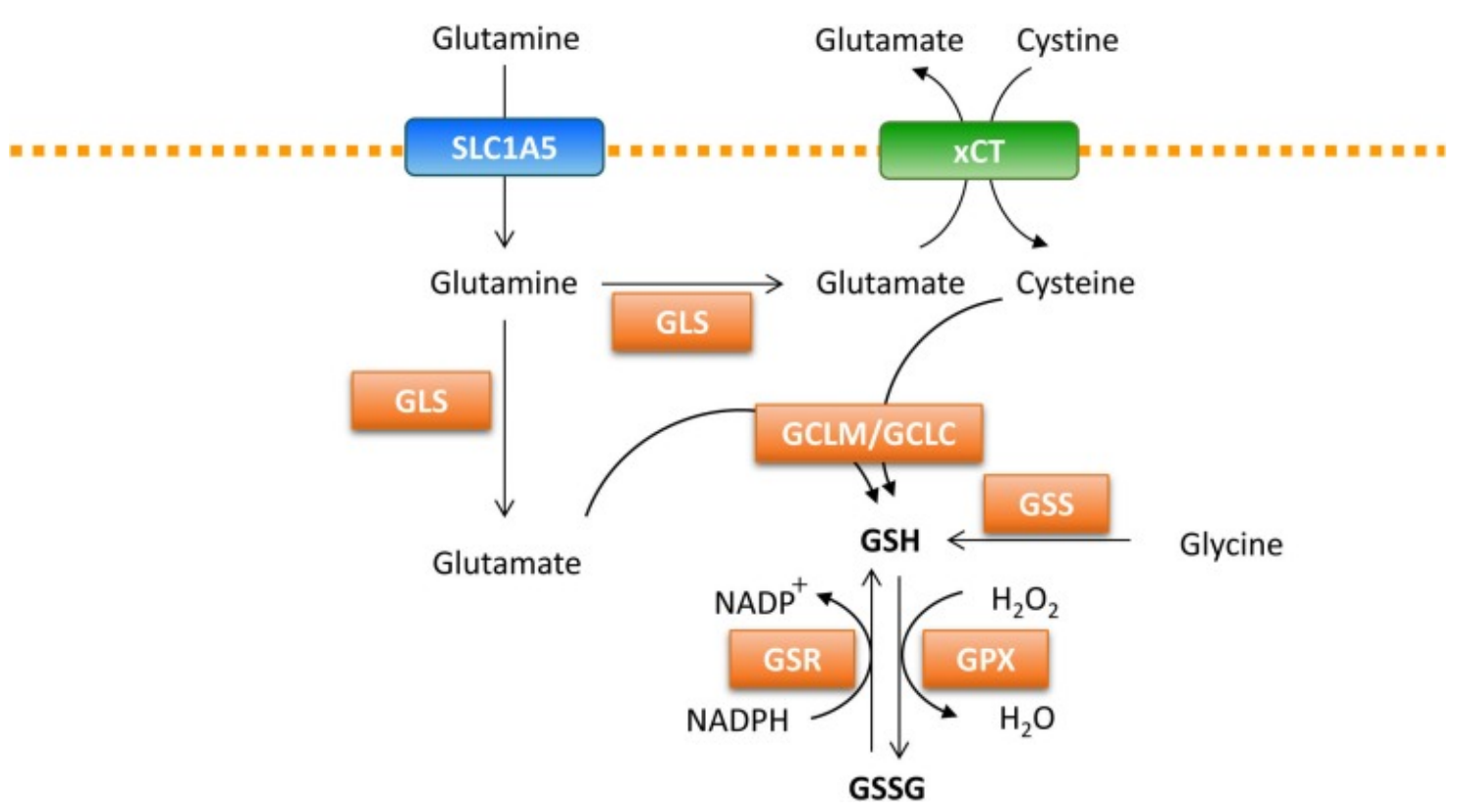

Figure 11. Glutamine metabolism and regulation of oxidative stress.

The conversion of glutamine to glutamate is catalyzed by glutaminase (GLS). Glutathione (GSH), a major antioxidant in mammalian cells, is a tripeptide composed of glutamate, glycine and cysteine. The first step in GSH synthesis is the ligation of glutamate and cysteine to form $\gamma$-glutamylcysteine, a process catalyzed by glutamate-cysteine ligase (GCL), which is a heterodimer composted of a modifier (GCLM) and a catalytic (GCLC) subunit. The second step in GSH synthesis is the coupling of $\gamma$-glutamylcysteine and glycine, which is mediated by glutathione synthetase (GSS). Glutathione peroxidase (GPX) converts hydrogen peroxide $\left(\mathrm{H}_{2} \mathrm{O}_{2}\right)$ to water $\left(\mathrm{H}_{2} \mathrm{O}\right)$, forming glutathione disulfide (GSSG), the oxidized form of GSH. GSSG can be converted back to GSH, through the conversion of NADPH to NADP+, in a reaction catalyzed by glutathione reductase (GSR). Figure from [163] 


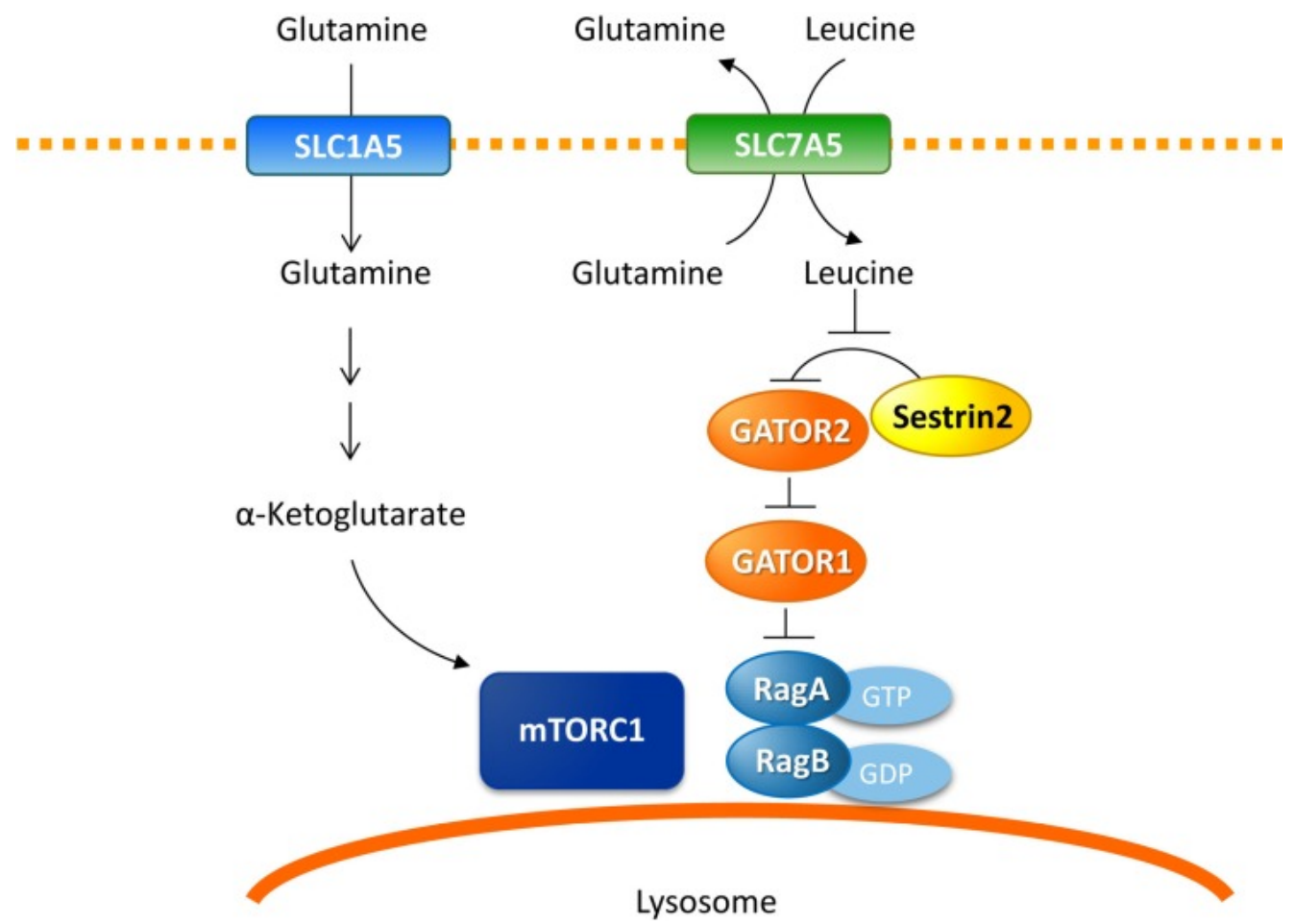

Figure 12. Activation of the mechanistic target of rapamycin complex 1 (mTORC1) by glutamine and leucine.

The active form of mTORC1 is localized in the surface of lysosomes, and $\alpha$-ketoglutarate stimulates the recruitment of mTORC1 to the lysosome. Additionally, the amino acid transporter SLC7A5 mediates leucine efflux in exchange with glutamine, and leucine has been shown to disrupt the binding between Sestrin2 and GATOR2, an inhibitor of mTORC1, thus promoting mTORC1 activation. Figure from [163] 


\section{Lipids}

Lipids in histotroph are hypothesized to play key roles in supporting embryonic development, because they serve as energy source and storage, provide substrates for membrane biogenesis, and act as signaling molecules [169].

The availability of lipids in uterine cells fluctuates throughout the estrus cycle in several species [170]. In sheep, lipid droplets in the surface of endometrial LE have been reported to be high during estrus, decreases during early diestrus (days 1-6), and as the corpus luteum develops and progesterone concentrations increases, increasing abundance of lipids are detected on the surface LE cells [171] (Figures 13 A). Similarly, concentrations of neutral lipids in the cytoplasm of endometrial epithelial cells increases substantially after day 8 of the estrus cycle in sheep (Figure 13 B). Thus, the available evidence suggests that onset of conceptus elongation (day 12) occurs when concentrations of lipids are increased in the endometrium epithelium and uterine lumen. Additionally, lipid droplets are reduced in the endometrium of pregnant than cyclic ewes between days 14 and 16 (Figure $13 \mathrm{~B}$ ), suggesting that the elongating conceptus is utilizing the LE uterine lipids to support its growth $[172,173]$.

Lipids likely participate in the crosstalk between the conceptus and endometrium during early pregnancy, as a diverse class of lipids can serve as ligands of peroxisome proliferator-activated receptors (PPARs) [172, 174-178]. Both the bovine conceptus and endometrium express PPARs [94, 179]. PPARs are ligand-regulated transcription factors that upon heterodimerization with retinoid $\mathrm{X}$ receptor $(\mathrm{RXR})$ and ligand binding, regulate the expression of target genes and consequently cellular function [175] (Figure 14). PPAR/RXR are involved in the transcriptional regulation of lipid uptake [180], fatty acid oxidation and metabolism [181], glucose homeostasis [182], and cell proliferation and 
differentiation [183], which are particularly important biological processes during conceptus elongation. Of note, PPARG expression increases during conceptus elongation in cattle, and its expression is correlated with the expression several genes involved in lipid metabolism [94].

Fatty acids are common components of complex lipids, and serve as major energy source during embryonic development [184]. In eukaryotes, fatty acid catabolism ( $\beta$ oxidation) occurs in the mitochondria, and generates acetyl-coA, $\mathrm{NADH}$ and $\mathrm{FADH}_{2}$, which are used in the TCA cycle and electron transport chain (ETC) for the synthesis of ATP (Figure 15) [185]. Additionally, fatty acids are precursors of important lipid mediators with critical function during early pregnancy, such as classical eicosanoids (prostaglandins, thromboxane, and leukotrienes) and lysophospholipids [120, 186]. Fatty acid biosynthesis and catabolism in mammalian cells involves dynamic processes in which the number of carbons and double bonds change under the influence of elongation, desaturation, and $\beta$ oxidation reactions. Fatty acid elongases (ELOVL) regulate fatty acid length by catalyzing carbon chain extensions [187], and desaturases controls the degree of fatty acid unsaturation, as it catalyzes the insertion of double bonds into the fatty acid chain [188] (Figure 16).

The transport of lipids from the endometrium to the uterine lumen are thought to be mediated by distinct mechanisms involving carrier proteins, lipoproteins, and extracellular vesicles (EVs) [189] (Figure 17). Albumin is the main fatty acid binding protein in extracellular fluids [190], and nearly $100 \%$ of the fatty acid detected in plasma are bound to albumin [191]. Albumin is also the most abundant protein in the uterine luminal fluid of cows [81] and sheep [192], and therefore possibly a major carrier of fatty acids to the uterine lumen. 
Available evidence suggests that lipids are also carried to the uterine lumen by lipoproteins (e.g. chylomicrons, very low density lipoprotein (VLDL), intermediatedensity lipoprotein (IDL), low-density lipoprotein (LDL), high density lipoprotein (HDL)) [193]. Argraves and Morales [194] suggested that high-density lipoprotein (HDL) is synthesized by the endometrium GE in rats, as their uterine glands express apoproteins $\mathrm{J}$ and A1, which are important components for HDL synthesis. Additionally, Koch et al. [195] observed that apoprotein A1 was more abundant in uterine fluid of pregnant than open ewes on day 16 of the estrus cycle/pregnancy. Importantly, the elongating bovine conceptus expresses lipoprotein lipase (LPL) [179], and therefore is able to hydrolyze lipoproteins for fatty acids uptake [196].

The mechanism of fatty acid uptake involves membrane-bound carrier proteins, such as membrane-associated fatty acid binding protein (FABPa), and scavenger receptors (SRs, CD36), as well as cytoplasmic FABPs, which receives the fatty acid from the membrane bound transporters and facilitates their transfer between extra- and intracellular membranes [197]. Expression of FABP3 in endometrial epithelial cells has been reported to decrease from days 13 to 19 of the estrus cycle but not during pregnancy in heifers [198], suggesting that the rate of fatty acid transport remains high during the pre- and periimplantation period of pregnancy.

Extracellular vesicles, which includes microvesicles and exosomes, are also thought to be an important lipid source during early pregnancy in ruminants [189]. EVs are lipid bilayer-enclosed nano-sized particles that are present in all body fluids, and can carry a large number of active molecules, such as lipids, proteins, and nucleic acids [199, 200]. The ovine conceptus and endometrium secrete EVs during early pregnancy, and the conceptus is able to incorporate uterine-derived EVs [201]. Thus, regardless of the cargo, 
the lipid bilayer of uterine-derived EVs by itself constitute an important source of complex lipids for the elongating conceptus, as the cellular membrane of EVs are enriched in cholesterol, sphingolipids, glycerophospholipids and ceramides [202]. 

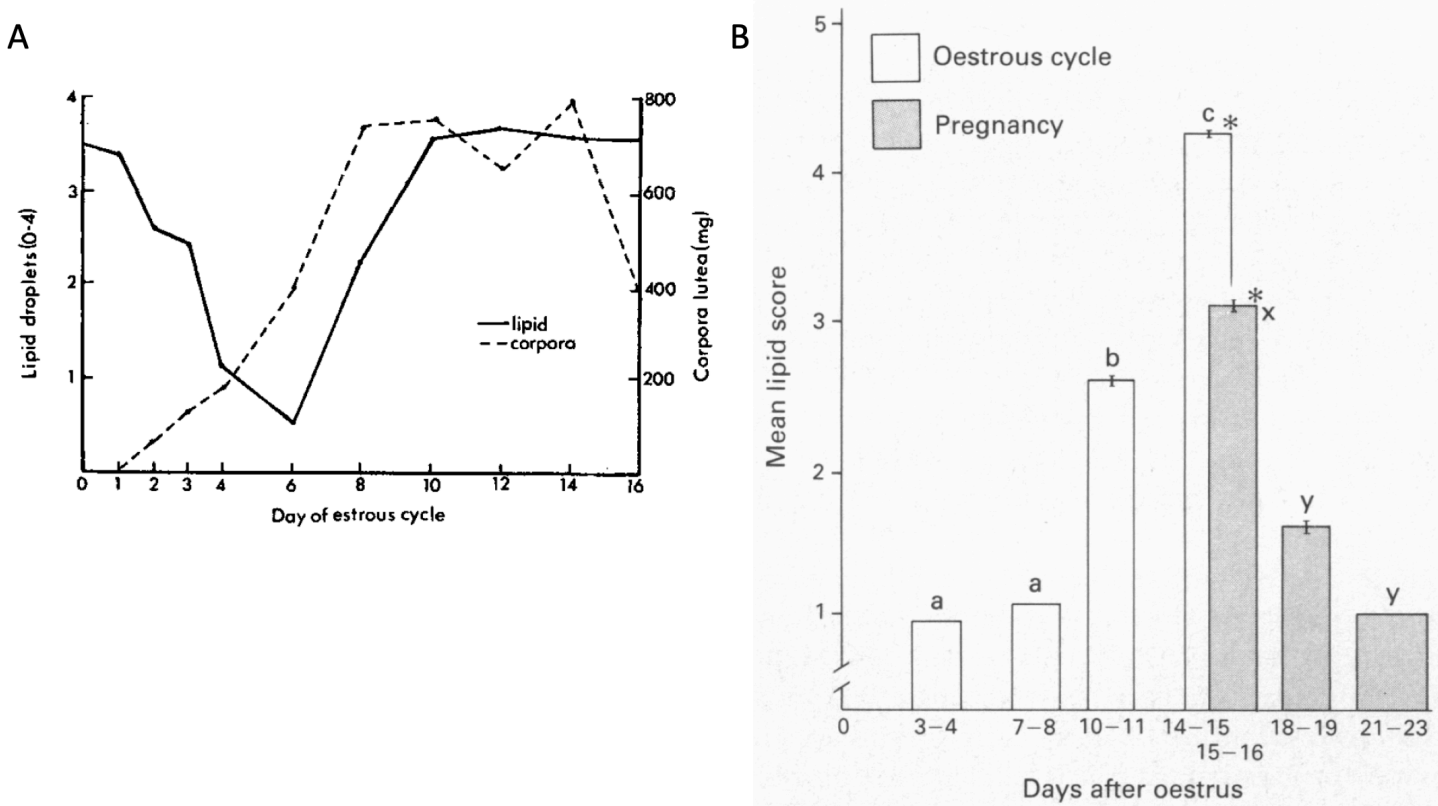

Figure 13. Lipid accumulation in the uterine lumen and within endometrial epithelial cells.

(A) Relationship between the abundance of lipid droplets (solid line) in the surface of uterine luminal epithelial (LE) cells and corpus luteum (CL) weight (dashed line) during the estrus cycle in sheep. The increase in CL volume is associated with increased concentrations of lipid droplets in the surface of uterine LE cells. Figure from [171]. (B) Mean lipid score in endometrial LE of cyclic and pregnant sheep. Concentrations of intraepithelial lipids are reduced in pregnant than cyclic endometrium between days 14 and 16, and endometrial lipids decreases as pregnancy advances, suggesting that the conceptus is utilizing uterine lipids to support its growth. Figure from [173]. 


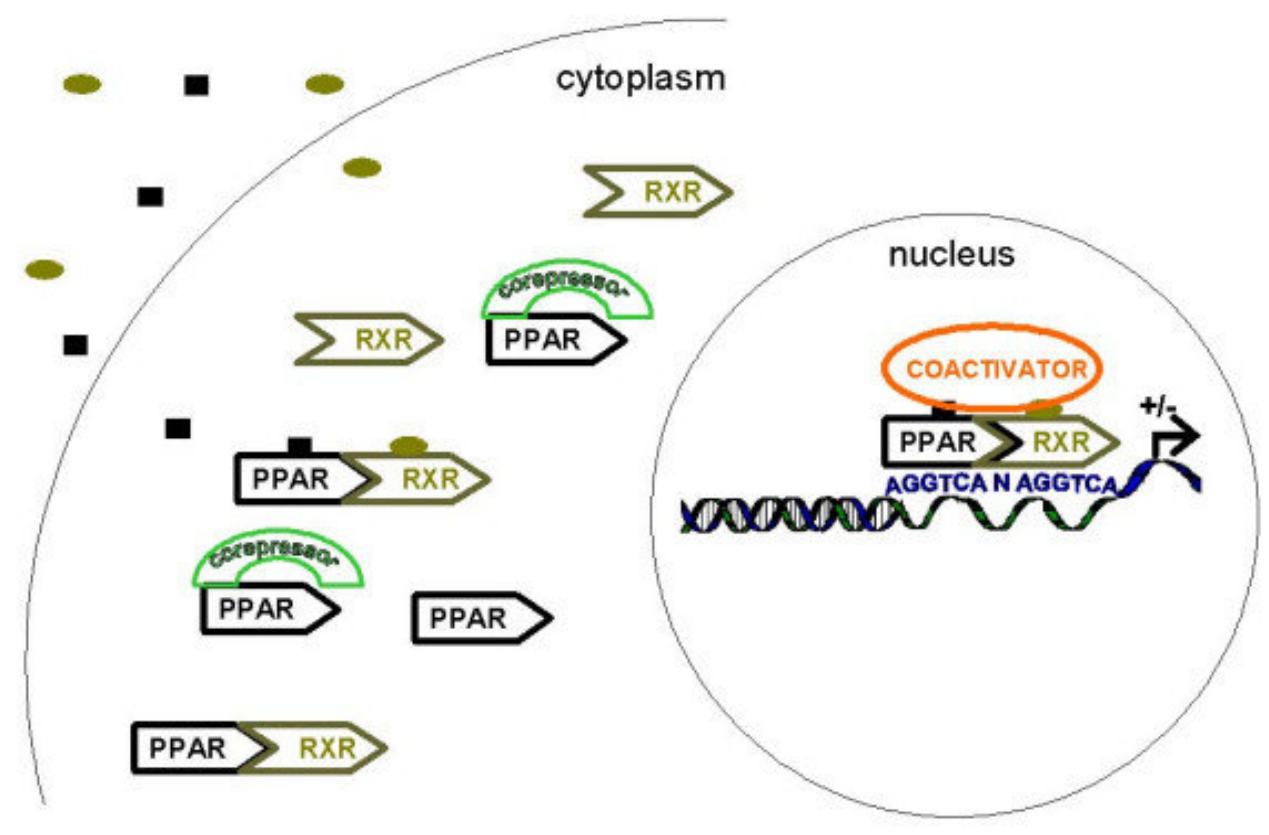

Figure 14. Mechanism of transcriptional activation of peroxisome proliferatoractivated receptors (PPARs).

PPARs can heterodimerize with retinoid X receptor (RXR) independently of ligand binding. Upon ligand binding, PPARs undergo conformational change dissociating from corepressors and recruiting co-activators. A diverse class of lipids can serve as ligands of PPARs. PPAR signaling is mediated through binding of PPAR/RXR heterodimers to PPAR response elements (PPRE) present in the promoter region of PPAR responsive genes, activating transcription. Cellular processes regulated by PPAR signaling include lipid metabolism, glucose homeostasis, and cell proliferation and differentiation. Figure from [203]. 


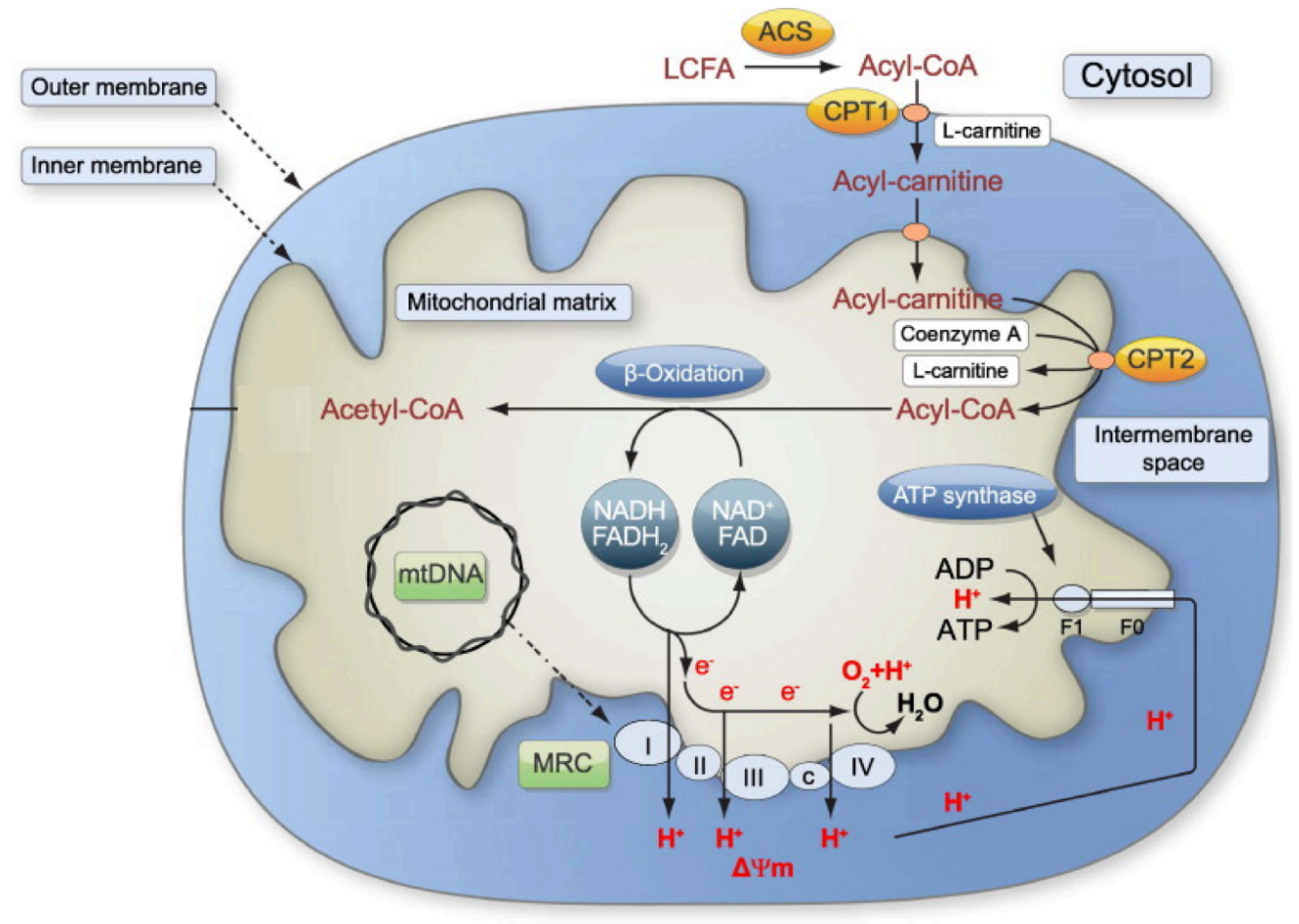

\section{Figure 15. Mitochondrial fatty acid $\beta$-oxidation.}

Long-chain fatty acids (LCFA; C11-C20) cannot diffuse through the mitochondrial membranes, thus a specific transport system is required for $\beta$-oxidation. First, LCFA are bound to coenzyme A by long-chain acyl-CoA synthetases (ACS) located in the outer mitochondrial membrane. Then, the enzyme carnitine palmitoyltransferase-1 (CPT 1) catalyzes the binding of long chain acyl-CoA with L-carnitine, forming acyl-carnitine, which can cross the mitochondrial inner membrane in a process mediated by carnitineacylcarnitine translocase. Finally, carnitine palmitoyltransferase-2 (CPT 2), an enzyme located on the matrix side of the inner mitochondrial membrane, breaks down acylcarnitine forming Acyl-CoA, coenzyme A and L-carnitine. Acyl-CoA is then oxidized in to Acetyl-CoA, in a process that generates $\mathrm{NADH}$ and $\mathrm{FADH}_{2}$, which are used in the electron transport chain (ETC) for the synthesis of ATP. Acetyl-CoA can also be directed to the TCA cycle for energy production. Figure adapted [185] 


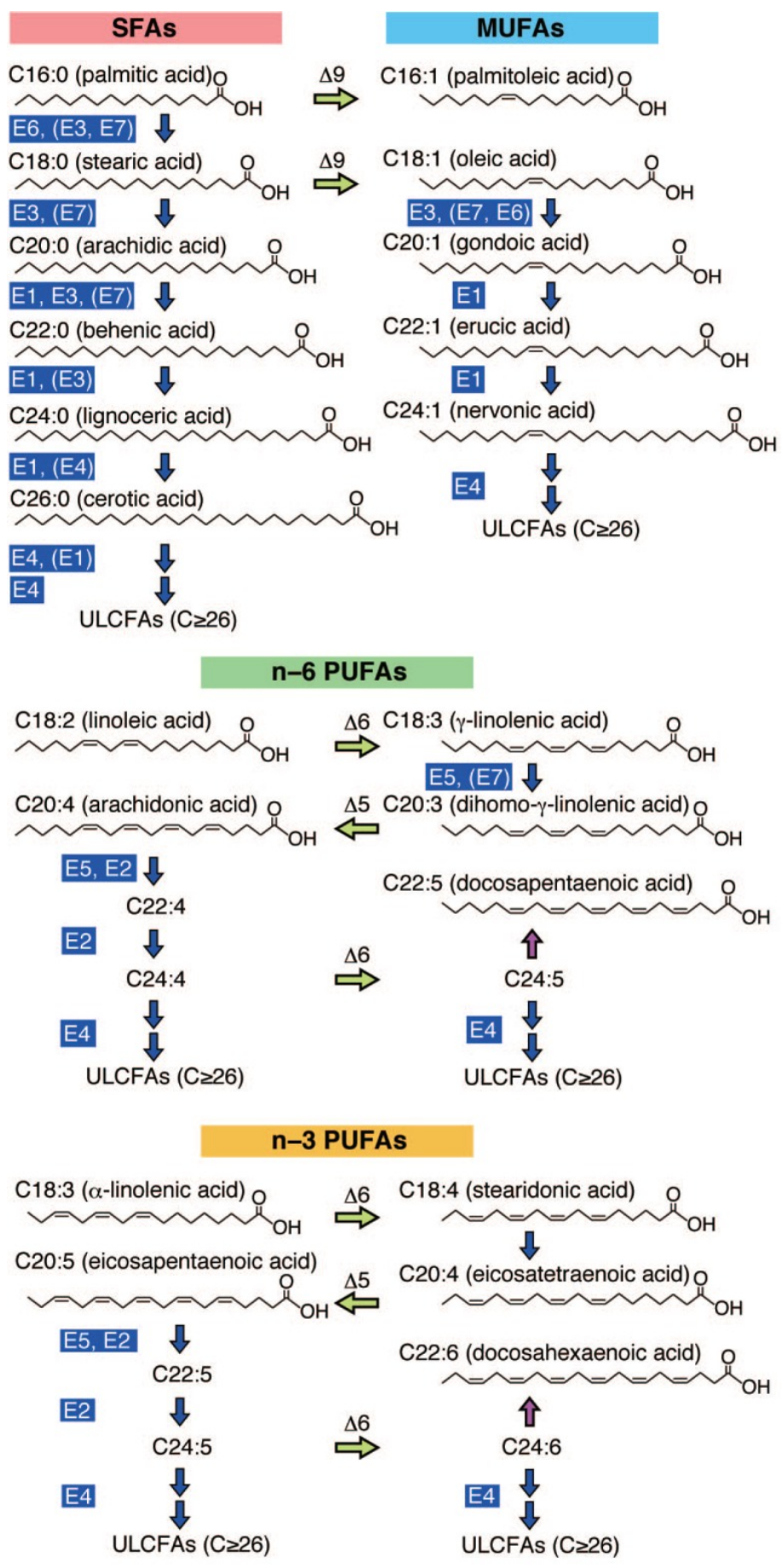

Лูelongation 』ु desaturation 』ु $\beta$-oxidation

\section{Figure 16. Fatty acid elongation and desaturation.}

Fatty acid biosynthesis and catabolism involves dynamic processes in which the number of carbons and double bonds change under the influence of elongation, desaturation and $\beta$ - 
oxidation reactions. ELOVL isozymes (E1-E7), are enzymes responsible for the elongation step, catalyzing carbon chain extension. Desaturases $(\Delta 5, \Delta 6$ and $\Delta 9)$ are enzymes that catalyzes the removal of 2 hydrogen atoms from fatty acids, creating carbon/carbon double bonds. Fatty acids are shortened by two carbons each round of $\beta$-oxidation, as they are released in the form of acetyl CoA. FA: fatty acid; SFA: saturated FA; MUFA: monounsaturated FA; PUFA: polyunsaturated FA. Figure from [188] 


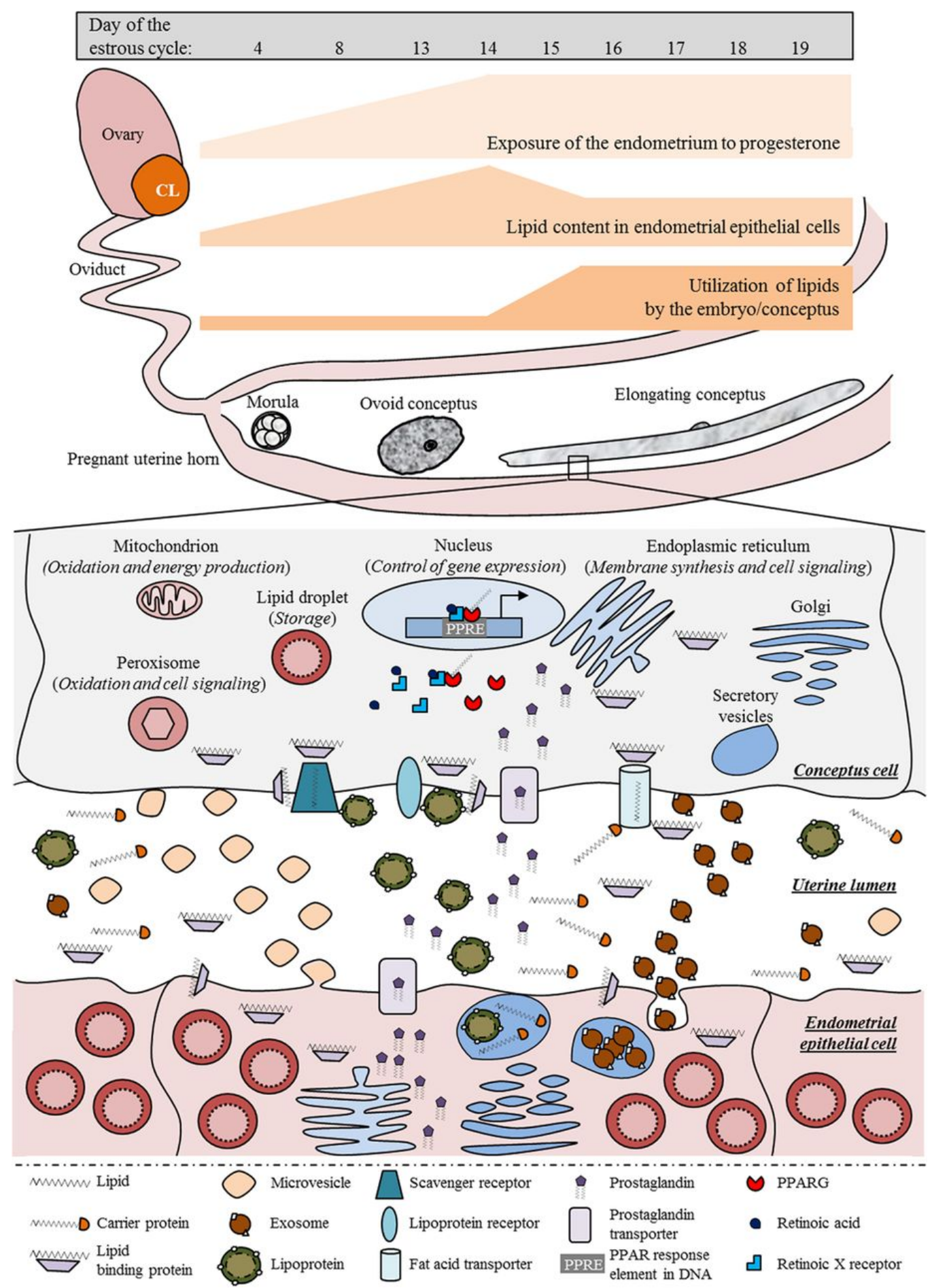

Figure 17. Proposed model for lipid metabolism during early pregnancy in cattle.

Ribeiro et al. [189]. Ovarian progesterone induces lipid accumulation in epithelial cells, which serve as lipid source to support conceptus development during early pregnancy. Endometrial lipids are transported to the uterine lumen by exosomes, microvesicles, 
lipoproteins and carrier proteins. The conceptus utilizes endometrial lipids as energy source, for membrane biogenesis, and for cell signaling. Conceptus PPARG activation by uterine derived lipids is essential to regulate conceptus gene expression to support elongation. 


\section{Prostaglandins}

Prostaglandins (PGs) are the most well-studied class of lipid mediators, and the ruminant uterus and conceptus secrete a variety of PGs [120, 204, 205]. The first step of PG synthesis (Figure 18) is the release of arachidonic acid from membrane phospholipids, a process mediated by phospholipase A2 (PLA2). Arachidonic acid can then be converted into PGG2 by prostaglandin synthases I (PTGS1/COX1) or II (PTGS2/COX2). PGG2 exits the active site of PTGS1/2 and undergoes a two-electron reduction, forming PGH2, which can be metabolized into the five primary prostanoids (PGD2, PGF2a, PGI2, thromboxane A2 (TXA2), and PGE2) through the action of specific PG synthases. Prostaglandin signaling is mediated by membrane-bound G protein-coupled receptors as well as nuclear receptors [206-208]. Membrane bound receptors includes PGD2 receptor (DP, or PTGDR),

PGF2 $\alpha$ receptor (FP or PTGFR), PGI2 receptor (IP or PTGIR), TXA2 receptor (TP or TBXA2R), and 4 subtypes of PGE2 receptors (EP1/PTGER1, EP2/PTGER2, EP3/PTGER3, and EP4/ PTGER4) [209-211]. Different types of PGs act through their specific receptor(s), and therefore regulates distinct biological processes $[212,213]$. The nuclear receptors involved with PG signaling includes PPARD and PPARG, as PGI2 can signals through PPARD [214], and 15-deoxy- $\Delta^{12,14}$-PGJ2, a metabolite of PGD2, can activate PPARG [215, 216].

Prostaglandin synthase 2 is the major PG synthase expressed by both, the conceptus and endometrium during early pregnancy in sheep. Charpigny et al. [205] demonstrated that PTGS2 was highly abundant in day 8 to 17 blastocysts/conceptuses, whereas PTGS1 was undetectable during this period. Loss of PGR in the endometrial LE/sGE around day 10 of the estrus cycle in sheep is associated with changes in endometrial gene expression required for successful implantation, and PTGS2 becomes upregulated in the endometrial 
LE/sGE during this period. Interferon tau secreted by the elongating conceptus further stimulates endometrial expression of PTGS2 [120, 205]. Thus, concentrations of PGs in the uterine lumen rises during early pregnancy in ruminants, as the endometrium and conceptus PG synthesis is upregulated during this period [138, 204].

Prostaglandin signaling in the endometrium is essential to establish uterine receptivity (Figure 19). In fact, PGs and IFNT stimulates a number of progesterone stimulated genes in the endometrial LE/sGE during early pregnancy, including glucose (SLC2A1, SLC2A5, SLC5A11) and amino acid (SLC7A2) transporters, secreted proteins (CST3, CTSL1, GRP, IGFBP1, LGALS15), and intracellular enzymes (HSD11B1) [217]. Intrauterine infusion of meloxicam, a selective inhibitor PTGS2, completely inhibits conceptus elongation in sheep [218], and endometrial expression of HSD11B1 and IGFBP1 is particularly reduced by meloxicam treatment [218, 219]. The enzyme hydroxysteroid 11- $\beta$ dehydrogenase 1 (HSD11B1), also known as cortisone reductase, catalyzes the conversion of inactive cortisone to active cortisol, which signals through the glucocorticoid receptor (GR) to stimulate a number of elongation and implantation-related genes [220, 221]. Cortisol also has immunosuppressive effects, which are thought to be important for maintaining the immunologic homeostasis required for the survival of the semiallogeneic conceptus/fetus during pregnancy [222]. IGF binding protein 1 (IGFBP1), another important gene upregulated by PGs in the endometrium, mediates IGF1 activity [223], and IGF1 is a major regulator of embryonic growth and development [224]. Interestingly, IGFBP1 also acts independently of IGF1 stimulating trophoblast migration and attachment in the human placenta [225]. Thus, the downstream effects of PGs in the endometrium are clearly important for successful attachment and implantation mammals. 
Angiogenesis and vascular permeability are among the essential processes regulated by PGs during early pregnancy. For instance, PGF2 $\alpha$ has been reported to increase the expression of vascular endothelial growth factor A (VEGFA) in the endometrial LE [226], and VEGFA is one of the most important proangiogenic factors, inducing vascular permeability, angiogenesis, and endothelial cell growth [227]. Additionally, PGE2 signaling through EP4 can stimulates angiogenesis [228, 229], and PGI2 signaling can increase vascular permeability by inducing vascular smooth muscle relaxation [230].

Brooks et al [120] proposed a model for the regulation of early pregnancy events in sheep by PGs and IFNT (Figure 19). During conceptus elongation, conceptus-secreted PGs may act in an autocrine manner through nuclear receptors (PPARD, PPARG) to increase the transcription of genes involved with cell proliferation and metabolism. Additionally, conceptus-secreted PGs may exit the TE through prostaglandin transporters (PGT and MRP4) to regulate endometrial expression of elongation and implantation related genes. Prostaglandins produced by endometrial epithelial cells may also act in an autocrine manner mediating the expression of genes to support conceptus elongation and implantation, as well as in a paracrine manner controlling vascularization and immunomodulation in the stroma and deep GE [120, 231] (Figure 19). Endometrialderived PGs may also activate conceptus-expressed membrane bound and nuclear receptors to further support conceptus development. 


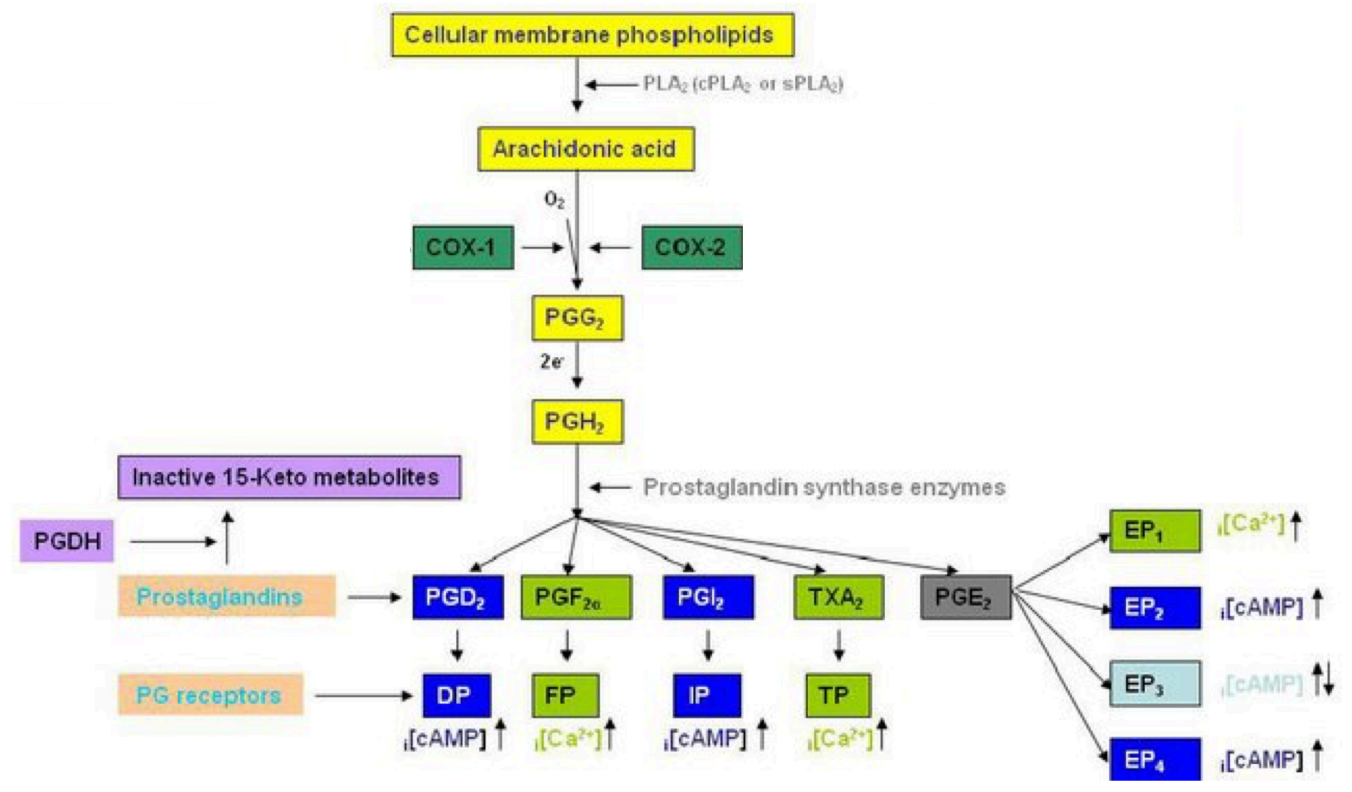

Figure 18. Prostaglandin synthesis from membrane phospholipids.

The first step of PG synthesis is the release of arachidonic acid from membrane phospholipids, a process mediated by phospholipase A2 (PLA2). Arachidonic acid can then sequentially be transformed into PGG2 by prostaglandin synthases I (PTGS1/COX1) and II (PTGS2/COX2). PGG2 then exits the active site of PTGS1/2 and undergoes a twoelectron reduction, forming $\mathrm{PGH} 2$, which can be metabolized into the five primary prostanoids (PGD2, PGF2a, PGI2, thromboxane A2 (TXA2), and PGE2) through actions of specific PG synthases. The classical signaling of PGs is mediated by membrane-bound $\mathrm{G}$ protein-coupled receptors as well as nuclear receptors. Membrane bound receptors includes PGD2 receptor (DP, or PTGDR), PGF2 $\alpha$ receptor (FP or PTGFR), PGI2 receptor (IP or PTGIR), TXA2 receptor (TP or TBXA2R), and 4 subtypes of PGE2 receptors (EP1/PTGER1, EP2/PTGER2, EP3/PTGER3, and EP4/ PTGER4). The second messengers regulating PG signaling for each specific receptor are presented. The enzyme 15hydroxyprostaglandin dehydrogenase (PGDH) catalyzes the first and rate-limiting step in the inactivation and degradation of PGs. Figure from [211] 


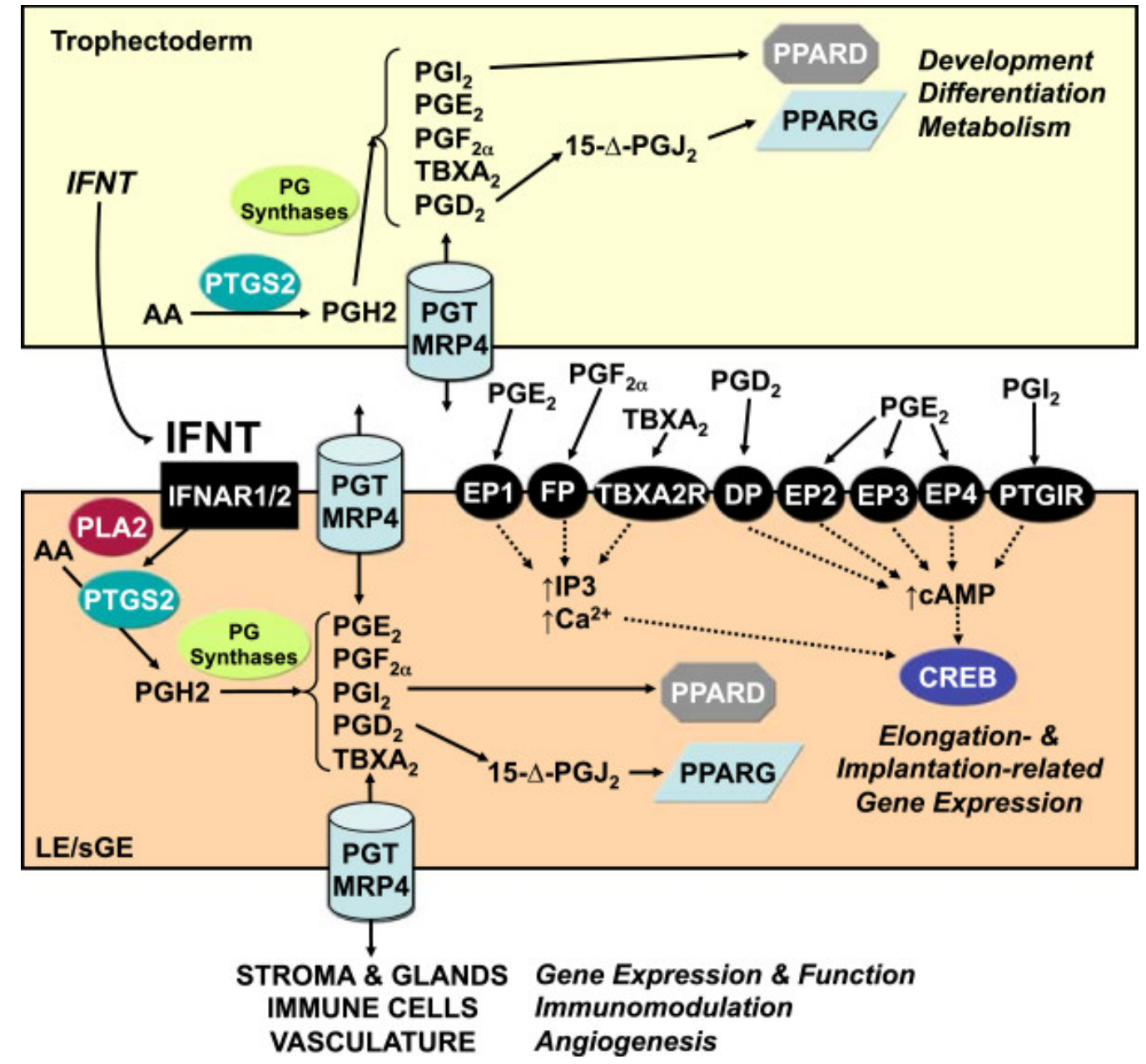

Figure 19. Proposed model for prostaglandin (PG) and interferon tau (IFNT) regulation of uterine function and conceptus elongation during early pregnancy in sheep.

Brooks et al [120]. Conceptus-secreted PGs may act in an autocrine manner through nuclear receptors (PPARD, PPARG) increasing the transcription of genes involved with cellular proliferation and metabolism. The expression of prostaglandin transporters (PGT and MRP4) by the conceptus facilitates the transport of conceptus-derived PGs into the uterine lumen. The endometrium LE/sGE express several classes of proteinoid receptors, and their signaling mediates the expression of elongation and implantation related genes. IFNT secreted by the elongating conceptus further increase the expression of prostaglandin 
synthase 2 (PTGS2) in the endometrial LE/sGE, increasing the overall synthesis of prostanoids, and consequently their signaling. PGs also signals through nuclear receptors, as PGI2 can activate PPAR delta (PPARD) [214], and 15-deoxy- $\Delta^{12,14}$-PGJ2, a metabolite of PGD2, can activate PPAR gamma (PPARG). Additionally, prostanoids are transported from endometrial LE/sGE into the stroma and deep GE, where they regulate important biological processes during early pregnancy such as angiogenesis, immunity. Legend: ABCC4, ATP-binding cassette, sub-family C (CFTR/MRP), member 4; CREB, cAMP responsive element binding protein; IFNAR, interferon (alpha, beta and omega) receptor; DP, prostaglandin D receptor (PTGDR); EP, prostaglandin E receptor (PTGER); FP, prostaglandin F receptor (PTGFR); IP, prostaglandin I receptor (PTGIR); PLA2, phospholipase A2; PPARD, peroxisome proliferator-activated receptor delta; PPARG, peroxisome proliferator-activated receptor gamma; PTGS2, prostaglandin-endoperoxide synthase 2 (prostaglandin G/H synthase and cyclooxygenase); PG Synthases, prostaglandin synthases (AKR1C3, PTGDS, PTGES, PTGFS, PTGIS, TBXAS); ; SLCO2A1 (also known as PGT), solute carrier organic anion transporter family, member 2A1 (prostaglandin transporter); TBXA2R, thromboxane A2 receptor. 


\title{
SECTION II:
}

\section{Identification of Beef Heifers with Superior Uterine Capacity for Pregnancy}

\author{
Thomas W. Geary, Gregory W. Burns, Joao G.N. Moraes, James I. Moss, Anna C. \\ Denicol, Kyle B. Dobbs, M. Sofia Ortega, Peter J. Hansen, Michael E. Wehrman, Holly \\ Neibergs, Eleanore O’Neil, Susanta Behura, and Thomas E. Spencer
}

This work has been published in:

Biology of Reproduction, Volume 95, Issue 2, 1 August 2016, 47, 1-12. doi:

10.1095/biolreprod.116.141390 


\section{ABSTRACT}

Infertility and subfertility represent major problems in domestic animals and humans, and the majority of embryonic loss occurs during the first month of gestation that involves pregnancy recognition and conceptus implantation. The critical genes and physiological pathways in the endometrium that mediate pregnancy establishment and success are not well understood. In Study One, predominantly Angus heifers were classified based on fertility using serial embryo transfer (ET) to select animals with intrinsic differences in pregnancy loss. In each of the four rounds, a single in vitro-produced high-quality embryo was transferred into heifers on day 7 post-estrus and pregnancy was determined on days 28 and 42 by ultrasound and then terminated. Heifers were classified based on pregnancy success as high fertile (HF), subfertile (SF), or infertile (IF). In Study Two, fertilityclassified heifers were resynchronized and bred with semen from a single high fertility bull. Blood samples were collected every other day from days 0 through 36 post-mating. Pregnancy rate was determined on day 28 by ultrasound, and was higher in HF $(70.4 \%)$ than in heifers with low fertility (36.8\%; SF and IF). Progesterone concentrations in serum during the first 20 days post-estrus were not different in non-pregnant heifers and also not different in pregnant heifers among fertility groups. In Study Three, a single in vivoproduced embryo was transferred into fertility-classified heifers on day 7 post-estrus. The uteri were flushed on day 14 to recover embryos, and endometrial biopsies were obtained from the ipsilateral uterine horn. Embryo recovery rate and conceptus length and area were not different among the heifer groups. RNA was sequenced from the day 14 endometrial biopsies of pregnant HF, SF and IF heifers ( $n=5$ per group) and analyzed by edgeR robust analysis. There were 26 differentially expressed genes (DEG) in the HF compared to SF endometrium, 12 DEG for SF compared to IF endometrium, and 3 DEG between the HF 
and IF endometrium. Many of the DEG encoded proteins involved in immune responses and are expressed in B cells. Results indicate that pre-implantation conceptus survival and growth to day 14 is not compromised in SF and IF heifers. Thus, the observed difference in capacity for pregnancy success in these fertility-classified heifers is manifest between days 14 and 28 when pregnancy recognition signaling and conceptus elongation and implantation must occur for the establishment of pregnancy. 


\section{INTRODUCTION}

Infertility and subfertility are important and pervasive problems in agricultural animals and humans. In ruminants, embryo mortality is a major factor affecting fertility and thus production and economic efficiency[232-235]. There is a high degree of pregnancy loss in cattle between fertilization and term, ranging from $40 \%$ to $56 \%[235$, 236]. The majority of embryonic loss (70-80\%) occurs in the first 3 weeks of pregnancy in cattle[235-239]. Embryo mortality is greater in non-lactating cows than heifers[237], and early pregnancy loss is even greater in lactating dairy cattle and can approach $40 \%$ [ 232 , 240, 241]. Infertility and subfertility also impact the embryo transfer (ET) industry[242]. Mean survival rate to calving following transfer of in vivo-derived embryos from superovulated donors ranges from $31 \%$ to $60 \%$ [243], whereas in vitro-produced embryo survival rate is lower and ranges from $30 \%$ to $40 \%[234,243]$. Failure of the embryo to survive and establish pregnancy is due to both embryonic and maternal factors[244]. Many of the pregnancy losses observed in natural or assisted pregnancies can be attributed to inadequate uterine receptivity, which can be defined as the physiological state of the uterus when conceptus growth and implantation for establishment of pregnancy is possible[245]. Understanding how embryo development is controlled is critical for determining ways to reduce the high rates of early embryonic mortality.

After fertilization (day 0), the zona pellucida-enclosed bovine embryo enters the uterus at the morula stage on days 4-5 of gestation and develops into a blastocyst. The spherical blastocyst hatches from the zona pellucida on days 7 to 10 and continues to grow, changing from spherical to ovoid in shape between days 12 and 14 during a transitory phase preceding elongation, after which it is termed a conceptus (embryo and associated extraembryonic membranes)[246]. The conceptus grows from about $2 \mathrm{~mm}$ in length on day 13 
of gestation to $6 \mathrm{~mm}$ on day 14, and reaches $60 \mathrm{~mm}$ by day 16 to $20 \mathrm{~cm}$ or more by day 19[247]. After days 16-17, the time of maternal recognition of pregnancy in cattle, the elongating conceptus begins the processes of implantation and placentation[248]. In both cattle and sheep, blastocyst growth into a conceptus and subsequent elongation has not been achieved in vitro and requires transfer into the uterus[249]. Progesterone action via the endometrium of the uterus is critical for conceptus growth and elongation in sheep and cattle[250-252]. Dynamic changes in endometrial gene expression occur between days 7 and 13 that are regulated by progesterone in nonpregnant and pregnant cattle and are associated with the onset of conceptus elongation[251-254]. A prevailing theory is that the gene expression changes modify the intrauterine milieu for support of the survival and growth of the blastocyst into an ovoid conceptus and then elongated filamentous conceptus. Uterine secretions in the lumen are not well defined in cattle, but are a complex mixture of proteins, amino acids, sugars, lipids and ions derived from genes expressed in the endometrium as well as selective transport of components (amino acids, glucose, albumin and other proteins) from maternal blood. Endometrial epithelial secretions are particularly important for conceptus survival and growth in ruminants, as uterine gland knockout (UGKO) ewes display recurrent early pregnancy loss due to defects in conceptus survival and elongation[255, 256]. Conceptus elongation is particularly critical for production of interferon tau (IFNT)[257, 258], the pregnancy recognition signal, that acts on the endometrium to sustain continued production of progesterone by the ovary and regulates genes implicated in implantation and placentation[245, 248, 259, 260]. Inadequate elongation of the conceptus results in low IFNT production, inability to maintain the corpus luteum, and early pregnancy loss[261]. Although much information is known about embryo development into a blastocyst from in vitro systems[262], post-hatching blastocyst 
survival and growth as well as conceptus elongation remains under investigated. Available evidence supports an unequivocal role for progesterone-dependent endometrial secretions of the uterus as primary regulators of conceptus survival, growth and development throughout pregnancy[251,263]. However, the essential endometrial genes and secretions that mediate survival and growth of the blastocyst and conceptus remain largely unknown in cattle[264, 265].

One of the major impediments to research on the physiology and genetics of early pregnancy success in cattle is the lack of animals with defined high and low rates of early pregnancy loss. McMillan and Donnison[266] summarized a unique approach for experimentally identifying high and low fertility in dairy heifers based on early pregnancy success. Contemporary yearling heifers $(n=155)$ received two in vitro-produced (IVP) embryos on 6 separate occasions during a 26-month period. Sixty days after transfer, pregnancy and the number of fetuses were determined ultrasonically and then pregnancies were terminated with the process was repeated 6 times. That approach identified 25 heifers with high (76\%) and low (11\%) aggregate pregnancy rates. Of note, a failure in the mechanism involved in conceptus elongation and maternal recognition of pregnancy was suggested to be the cause of early pregnancy loss in the low fertility heifers[266, 267]. A similar approach was used here to fertility classify beef heifers based on natural variation in early pregnancy success. A series of studies were then conducted on fertility classified heifers to begin determining the physiological and genetic factors underpinning early pregnancy loss in beef cattle.

\section{MATERIALS AND METHODS}

\section{Animals}


All animal procedures were conducted in accordance with the Guide for the Care and Use of Agriculture Animals in Research and Teaching and approved by the Institutional Animal Care and Use Committees of the USDA-ARS Fort Keogh Livestock and Range Research Laboratory, Washington State University, and the University of Missouri.

\section{In Vitro Production of Embryos (Study 1)}

Embryos were produced in vitro at the University of Florida to obtain blastocysts for transfer into recipients. Oocytes obtained from genetically-undefined ovaries ( $\mathrm{n}=1699$ 2800 per replicate) were fertilized with frozen-thawed spermatozoa pooled from three bulls of various breeds. The total number of bulls used in the 8 replicates was 20 and represented Angus ( $\mathrm{n}=7)$, Limousin $(\mathrm{n}=3)$, Brangus $(\mathrm{n}=3)$, Simmental $(\mathrm{n}=2)$, unknown $(\mathrm{n}=2)$, Hereford $(\mathrm{n}=2)$ and Polled Hereford $(\mathrm{n}=1)$ sires.

The procedures for in vitro production were as follows. Cumulus oocyte complexes (COCs) were obtained by cutting the surface of each ovary with a scalpel and vigorously rinsing the ovary through a bath of oocyte collection medium (tissue culture medium-199 with Hank's salts (Hyclone, Logan, UT, USA), 2\% (v/v) of either bovine steer serum (PelFreez, Rogers, AR, USA) or adult bovine serum (Thermo Scientific HyClone, Logan, UT, USA), $2 \mathrm{U} / \mathrm{ml}$ heparin, $100 \mathrm{U} / \mathrm{ml}$ penicillin-G, $0.1 \mathrm{mg} / \mathrm{ml}$ streptomycin and $1 \mathrm{mM}$ glutamine). Groups of $10 \mathrm{COCs}$ were matured in $50 \mu \mathrm{l}$ droplets of oocyte maturation medium (tissue culture medium-199 with Earle's salts (Invitrogen, Carlsbad, CA, USA), $10 \%(\mathrm{v} / \mathrm{v})$ bovine steer serum or adult bovine serum, $2 \mu \mathrm{g} / \mathrm{ml}$ estradiol $17-\beta, 20 \mu \mathrm{g} / \mathrm{ml}$ bovine follicle stimulating hormone (Bioniche Life Sciences, Belleville, Ontario, Canada), $22 \mu \mathrm{g} / \mathrm{ml}$ sodium pyruvate, $50 \mu \mathrm{g} / \mathrm{ml}$ gentamicin sulfate and $1 \mathrm{mM}$ glutamine) covered 
with mineral oil for $20 \mathrm{~h}$ at $38.5^{\circ} \mathrm{C}$ and in a humidified atmosphere of $5 \%(\mathrm{v} / \mathrm{v}) \mathrm{CO}_{2}$. Sperm were purified by centrifugation over a Percoll@ (GE Healthcare, Pittsburgh, PA, USA) gradient (500 $\mu$ l of a solution of 45\% (v/v) Percoll (diluted 1:1 in HEPES-SOF) on top of $500 \mu 1$ of a solution of $90 \%$ (v/v) Percoll [268]. Up to 250 matured oocytes were fertilized in a $35-\mathrm{mm}$ dish with Percoll-purified sperm $\left(1.0 \times 10^{6} / \mathrm{ml}\right)$ for $8-10 \mathrm{~h}$ at $38.5^{\circ} \mathrm{C}$ in $1.7 \mathrm{ml}$ of synthetic oviduct fluid-fertilization medium (SOF) [269]. Cumulus cells were denuded after fertilization by vortexing in $600 \mu \mathrm{l}$ HEPES-TALP containing 10,000 $\mathrm{U} / \mathrm{ml}$ hyaluronidase. Putative zygotes were then cultured in $50 \mu 1$ microdrops of synthetic oviduct fluid-bovine embryo 2 (SOF-BE2) covered with mineral oil at $38.5^{\circ} \mathrm{C}$ in a humidified atmosphere of $5 \%(\mathrm{v} / \mathrm{v}) \mathrm{O}_{2}$ and $5 \%(\mathrm{v} / \mathrm{v}) \mathrm{CO}_{2}$ with balance $\mathrm{N}_{2}$. Cleavage rate was assessed at day 3 post-fertilization.

At day 4 or 5 post-fertilization, embryos were prepared for transport to Montana by placement of advanced embryos in groups of $\sim 50$ per tube in polypropylene-stoppered 10x63 mm polystyrene tubes containing $1 \mathrm{ml}$ SOF-BE2 supplemented with $1 \mathrm{mM}$ HEPES, pH 7.5 and $50 \mu \mathrm{M}$ dithiothreitol. Tubes had been equilibrated overnight in a humidified incubator at $38.5^{\circ} \mathrm{C}, 5 \% \mathrm{O}_{2}, 5 \% \mathrm{CO}_{2}$ and $90 \% \mathrm{~N}_{2}$. After addition of embryos to the modified SOF-BE2, $\sim 1.5 \mathrm{ml}$ mineral oil was layered over the medium and tubes were placed in a Biotherm portable incubator (Cryologic, Blackburn, Victoria, Australia) at $38.5^{\circ} \mathrm{C}$. Embryos were hand carried to Montana by airliner on day 5 or 6 . Embryos were transferred to fresh $50 \mu 1$ drops of SOF-BE2 and cultured at $38.5^{\circ} \mathrm{C}$ in a humidified atmosphere of of $5 \%(\mathrm{v} / \mathrm{v}) \mathrm{O}_{2}$ and $5 \%(\mathrm{v} / \mathrm{v}) \mathrm{CO}_{2}$ with balance $\mathrm{N}_{2}$ until day 7 (embryos shipped on day 4) or were allowed to develop in the shipping tubes until day 7 (embryos shipped on day 5). 


\section{Embryo Transfer (ET) and Pregnancy Determination by Ultrasonography (Study 1)}

Crossbred beef heifers ( $\mathrm{n}=275$ Angus $\mathrm{x}$ Polled Hereford) were identified from a single population of cows at the USDA-ARS, Fort Keogh Livestock and Range Research Station and reared on a typical replacement heifer feedlot ration. Six heifers were culled at weaning based on extremely small body size. As illustrated in Figure 1, the remaining heifers were subjected to serial estrous synchronization and ET to classify heifers into fertility categories based on pregnancy outcome. Estrous cycles of heifers were synchronized beginning at approximately 14 months of age when heifers weighed $368 \pm$ $2.8 \mathrm{~kg}$ and had a body condition score of $5.9 \pm 0.1$ (scale of 1 to $9: 1=$ emaciated and $9=$ obese) to prepare heifers for ET on day 7 after estrus (day 0). Estrus was synchronized using the PG-6d-CIDR protocol which includes prostaglandin F2 alpha (PGF) administration (Lutalyse, Zoetis Animal Health, Madison, NJ) on day -12, an injection of GnRH (100 $\mu \mathrm{g}$, IM; Factrel, Zoetis Animal Health, Madison, NJ) concurrent with a intravaginal progesterone insert (CIDR) on day -9 , CIDR removal and PGF on day -3 , and estrus on day 0. Estrus detection patches (Estrotect, Rockway, Inc., Spring Valley, WI) were affixed to the tail head of each heifer to aid in visual detection of estrus. Heifers were observed for signs of estrus 3 times a day beginning $24 \mathrm{~h}$ after CIDR removal. Heifers that did not exhibit standing estrus received GnRH (100 $\mu \mathrm{g}$, IM; Factrel, Zoetis Animal Health, Madison, NJ) on day 0. During each ET round, heifers were randomly assigned to one of two groups on consecutive days to facilitate animal handling. All heifers received an in vitro produced embryo of high quality (blastocyst or expanded blastocyst) produced at the University of Florida on day 7 after estrus or induced ovulation by one of two experienced technicians. Ultrasonography was used to identify side and presence of the corpus luteum before ET by a single technician using an Aloka SSD 3500V and 7.5 MHz convex 
transducer (Aloka, Wallingford, CT). The embryo was placed in the uterine horn ipsilateral to the ovary containing a corpus luteum using standard non-surgical techniques. Heifers without a corpus luteum on day 7 did not receive an embryo.

Heifers were diagnosed for pregnancy on days 28 and 44 using transrectal ultrasonography with an Aloka SSD 500V and 7.5 MHz (day 28) or $5.0 \mathrm{MHz}$ (day 42) linear transducer. Viability was confirmed at each ultrasound by presence of a fetal heartbeat. At the second pregnancy diagnosis on day 44 , heifers received a single injection of PGF and were allowed a minimum of 30 days to exhibit another estrous cycle before being synchronized for another round of ET. After three or four rounds of ET, Heifers were classified based on day 28 pregnancy success as high fertile (HF; 100\%), subfertile (SF; 25-33\%), or infertile (IF; $0 \%$ ) based on results of 3 or 4 rounds of ET (Fig. 1).

\section{Blood Collection and Progesterone Radioimmunoassay for the Initial Round of ET} (Study 1)

Blood was collected from coccygeal vessels of heifers at the onset of synchronization (day -22 and -12) for the initial round of ET to assess serum concentration of progesterone as an indicator of pubertal status. Blood samples were allowed to clot for $1 \mathrm{~h}$ at room temperature, and then incubated at $4^{\circ} \mathrm{C}$ for approximately $24 \mathrm{~h}$. Samples were centrifuged at $1,200 \mathrm{x}$ g for $25 \mathrm{~min}$ at $4^{\circ} \mathrm{C}$ and serum was collected and stored at $-20^{\circ} \mathrm{C}$ until radioimmunoassays were performed. Radioimmunoassay (RIA) was performed on serum samples to measure progesterone concentrations using the Coat a Count RIA kit (Siemens, Malvern, PA)[270]. Inter- and intra-assay CV for a sample with $1.5 \mathrm{ng} / \mathrm{ml}$ were $11.45 \%$ and $11.79 \%$, respectively and assay sensitivity was $0.08 \mathrm{ng} / \mathrm{ml}$. 


\section{Estrous Synchronization and Artificial Insemination (Study 2)}

Estrous Synchronization and Pregnancy Determination. Estrous cycles of fertilityclassified heifers were synchronized using the PG-6d-CIDR protocol described in Study 1. Artificial insemination was performed at $12 \mathrm{~h}$ and $24 \mathrm{~h}$ after onset of standing estrus with semen from a single bull of known high fertility. Pregnancy diagnosis was performed by transrectal ultrasonography (SonoSite EDGE equipped with a L52 10.0-5.0 MHz lineararray transducer; SonoSite Inc., Bothell, WA) on days 28 and 36 post-AI.

Blood Sampling and Serum Progesterone Determination. Blood samples for determination of circulating progesterone concentrations were obtained by coccygeal venipuncture using evacuated tubes without anticoagulant (Becton Dickinson Vacutainer Systems, Franklin Lakes, NJ, USA) at time of breeding (day 0) and every other day from day 0 through day 28. Blood samples from heifers determined to be pregnant at day 28 were also collected every other day from day 28 through day 36 . Serum tubes were allowed to clot overnight at $4{ }^{\circ} \mathrm{C}$ then centrifuged at $3,000 \times$ g for 20 min at $4{ }^{\circ} \mathrm{C}$, and serum was collected and frozen at $-20^{\circ} \mathrm{C}$ until analyzed. Serum concentrations of progesterone were determined in triplicate $100 \mu \mathrm{l}$ aliquots of sample using manufacturer (MP Biomedical, Santa Ana, CA) reagents and recommendations for the liquid-liquid phase double antibody precipitation assay (MP Biomedicals cat\# 07-170105), and validated as previously described[271]. Inter- and intra-assay coefficients of variation were $<10 \%$.

\section{Embryo Transfer and Nonsurgical Uterine Flush on Day 14 (Study 3)}

Synchronization and Embryo Transfer. Estrous cycles of donor cows $(\mathrm{n}=7)$ and fertilityclassified heifers were tightly synchronized to optimize synchrony of embryos with uterine 
environment of recipient heifers. All females received PGF on day -18 to pre-synchronize estrous cycles and increase ovulation response (follicular wave reset) to $\mathrm{GnRH}$ administration on day -15 . On day -9 , all females received an intravaginal progesterone insert (CIDR) to supplement blood progesterone to assist preparation of the uterus for pregnancy and an injection of $2.5 \mathrm{mg}$ estradiol and $50 \mathrm{mg}$ progesterone to regress all follicles larger than $0.5 \mathrm{~mm}$ and again reset follicular wave growth. Donor cows received twice daily injections of follicle stimulating hormone (Folltropin; Agtech, Inc., Manhattan, KS) in decreasing daily doses from day -4.5 to -1 to stimulate multiple follicular development. The CIDRs were removed and PGF administered on day -2.5 (recipients) and -1.5 (donors) to synchronize estrus. Donors were inseminated $12 \mathrm{~h}$ and $24 \mathrm{~h}$ after onset of estrus (day 0 and 0.5 ) with semen from the same sire. Recipient heifers that did not exhibit estrus by day 0 received GnRH and estrogen ( $1 \mathrm{mg}$ estradiol benzoate). On day 7, each heifer received an in vivo-produced embryo placed in the uterine horn ipsilateral to the ovary containing a corpus luteum (as determined by ultrasound) using standard nonsurgical techniques by a single technician. Ultrasonography was used to identify side and presence of the corpus luteum before ET. Three heifers did not receive embryos due to excess fluid in their uterus or lack of a CL on their ovaries. Transferred embryos were of excellent quality and either a blastocyst $(n=53)$ or morula $(n=8)$ produced through superovulation of 7 donor cows. All transfers were frozen-thawed direct transfer embryos blocked by donor and assigned to heifers equally across fertility classification group.

Nonsurgical Uterine Flush. The uterus of each heifer was flushed to recover embryos at 7 days post-transfer using non-surgical techniques. Briefly, a 2-way luer-lock catheter (cat. no. 19982/0104, Minitube of America, Verona, WI) was passed into the uterine horn 
ipsilateral to the corpus luteum and $20 \mathrm{ml}$ of flush medium (Vigro complete flush medium; Agtech, Inc., Manhattan, KS) was placed into the uterine horn. After approximately $30 \mathrm{sec}$ of gentle massage, the initial flush medium was recovered in a syringe, transferred to a petri dish and searched for a conceptus. If a conceptus was not observed in the initial flush, additional medium ( $1 \mathrm{~L}$ ) was subsequently used to thoroughly flush the uterine horn. The flushes were accumulated and filtered through a $75 \mathrm{~m}$ mesh nylon filter (VCI Filter, Agtech, Inc.) then rinsed with flush medium and searched again for an embryo.

Endometrial Biopsy. Following uterine flush, an endometrial biopsy was collected near the greater curvature of the uterine horn ipsilateral to the ovary containing the corpus luteum. Endometrial tissue was obtained using Jackson uterine biopsy forceps (GerMedUSA Inc., Garden City Park, NY). The forcep was sterilized prior to biopsy collection using a hot bead sterilizer (Fine Science Tools (USA), Inc., Foster City, CA). To collect the endometrial biopsy, the forcep was protected with a sanitary chemise (IMV Technologies, Maple Grove, MN), which was broken immediately before the biopsy tool was passed through the cervical os. The tip of the forceps was directed to the greater curvature of the ipsilateral uterine horn, and endometrial tissue (biopsy) was collected by closing the instrument jaw. The forcep was then removed, and the biopsy sections were immediately placed in $1.5 \mathrm{ml}$ microcentrifuge tubes, frozen with liquid nitrogen, and stored at $-80^{\circ} \mathrm{C}$ until RNA extraction.

Conceptus Morphology and Measurement. Embryos were imaged on a Zeiss Discovery V8 stereomicroscope with an AxioCam ICC 1 and AxioVision version 4.6 software. 
Measurements of conceptus size (length and width) were collected using ImageJ (NIH, version 1.48).

\section{RNA Sequencing}

RNA Isolation. Total RNA was extracted from day 14 endometrial biopsies using the RNeasy Mini Kit (Qiagen, Valencia, CA). Briefly, frozen biopsy samples were disrupted and homogenized in RLT buffer with the use of a homogenizer (VDI 25, VWR International, Radnor, PA) and total RNA purified following the manufacturer's instructions. To eliminate DNA contamination, RNA was treated with DNase I during RNA purification using the RNase-Free DNase Set (Qiagen, Valencia, CA). RNA concentration was determined by quantitative high sensitivity RNA analysis on the Fragment Analyzer instrument (Catalog \# DNF-472, Advanced Analytical Technologies, Inc., Ankeny, IA). RNA library preparation and sequencing was conducted by the University of Missouri DNA Core facility.

Illumina TruSeq RNA Library Preparation and Sequencing. High-throughput sequencing services were performed at the University of Missouri DNA Core Facility. Libraries were constructed following the manufacturer's protocol with reagents supplied in Illumina's TruSeq mRNA stranded sample preparation kit. Briefly, the polyadenylated mRNA was purified from total RNA and fragmented. Double-stranded cDNA was generated from fragmented RNA, and the index containing adapters ligated. The final construct of each purified library was evaluated using the Fragment Analyzer instrument, quantified with the Qubit fluorimeter using the quant-iT HS dsDNA reagent kit (Invitrogen), and diluted 
according to Illumina's standard sequencing protocol for sequencing on an Illumina HiSeq 2500 sequencer.

\section{RNA-Seq Data Analysis}

Raw sequence reads (fastq) were trimmed of adapter sequences using cutadapt [272] followed by a windowed adaptive trimming step for base quality of the reads. The quality trimming was performed using Sickle (https:/github.com/najoshi/sickle) with default parameters for paired-end reads. The read data was deposited in the Gene Expression Omnibus (GSE81449). The trimmed paired-end reads were then mapped to the bovine reference genome UMD3.1.1 using SubRead aligner[273] followed by quantifying the number of reads mapped to exon sequences of annotated genes using FeatureCounts[274]. Genes significantly differentially expressed (FDR P $<0.10$ ) between samples were determined by fitting the read counts to a generalized linear model implemented in edgeR-robust[275]. The fold-changes of differential expression were determined from $\log _{2}(\mathrm{CPM})$ (counts per million reads), and the length-normalized FPKM (fragments per kilobase of exon per million reads) was determined from the sum of exon lengths of each gene. All statistical analyses and plotting were performed in R. The Pearson correlation coefficients were measured in pair-wise manner among samples to plot the correlation heat map of gene expressions. Conditional boxplots were generated using the Rlab package. The expression data of all genes across all the samples were used in exploratory maximum likelihood factor analysis by fitting the data to the factor analysis model using 'varimax' rotation option of 'factanal' in R. The plot was drawn using print method of R 'stats'. Violin plots were generated using ggplot2. 


\section{Statistical Analyses}

Statistical analyses were conducted using SAS (SAS Institute Inc., Cary, NC). Statistical significance was defined as $\mathrm{P}<0.05$.

Study 2. Pregnancy success to AI at days 28 and 36 post-insemination was analyzed by logistic regression with Firth's bias correction using the LOGISTIC procedure. The proportions of heifers pregnant at days 28 and 36 were determined using FREQ procedure. Progesterone concentrations were analyzed by ANOVA for repeated measures using MIXED procedure. For the repeated measures, models included the effects of fertility classification, day of sample collection, the interaction between fertility classification and day of sample collection, and the random effect of heifer nested within fertility classification. For all analysis, orthogonal contrasts were used to compare groups with high (HF) versus low fertility (SF and IF) and to compare differences between the groups with low fertility (SF vs IF).

Study 3. Day 14 embryo recovery rates were analyzed by logistic regression with Firth's bias correction using the LOGISTIC procedure. The proportions of embryos recoveried at day 14 flush were determined using FREQ procedure. Conceptus measurement data was subjected to least-squares analyses of variance (ANOVA) using the General Linear Models (GLM) procedure. Conceptus length, area and conception rate were tested for differences between fertility groups with embryo donor used as a covariate. Error terms used in the test of significance were identified according to the expectation of the mean squares for error. For all analysis, orthogonal contrasts were used to compare groups with high (HF) versus 
low fertility (SF and IF) and to compare the differences between the groups with low fertility (SF vs IF).

\section{RESULTS}

\section{Heifer Fertility Classification Using Embryo Transfer (Study 1)}

A total of 275 heifers were identified for the study, and 6 were culled due to extremely small size at weaning. Of the 269 heifers, 4 heifers were removed due to anovulation, death, health, or inability to pass the cervix. At the onset of synchronization for the first round of ET, 10 heifers were not cycling based on serum progesterone concentrations less than $1.0 \mathrm{ng}$ per $\mathrm{ml}$ in two samples collected 10 days apart. Estrous cycles were induced in 8 of 10 of these heifers with synchronization based on serum progesterone concentration on day 7 at ET. One of the anestrous heifers never began estrous cycles and was removed from the study; however, another anestrous heifer was pregnant each time she received an embryo and remained in the study.

In each round of ET, a single IVP embryo of high quality was transferred into heifers on day 7 post-estrus, and pregnancy determined on days 28 and 44 by ultrasound followed by pregnancy termination on day 44 (Fig. 1). Across the rounds of ET, 37 heifers did not have a CL at least once, and 5 heifers did not have a CL on two occasions. Pregnancy rates for each round of ET averaged $55 \%$ and $48 \%$ when diagnosed on day 28 and 44, respectively. Overall, serial ET was conducted in 260 heifers, and 4 rounds of ET data were generated on 228 heifers and 3 rounds of ET data on 32 heifers. As illustrated in Figure 1, this approach was use to classify heifers as high fertile (HF; $100 \%, \mathrm{n}=30$ ), subfertile (SF; $25-33 \%, \mathrm{n}=53)$, or infertile (IF; $0 \%, \mathrm{n}=12$ ) based on day 28 pregnancy success. 


\section{Pregnancy Rates after AI and Circulating Progesterone Concentrations (Study 2)}

All HF and IF heifers and a subset of SF heifers were synchronized to estrus and bred $12 \mathrm{~h}$ and $24 \mathrm{~h}$ post-estrus with semen from a single bull of known high fertility. Pregnancy was determined on days 28 and 36 by ultrasound. Blood samples were collected every other day beginning on day 0 through 28 in all heifers and through day 36 postmating in pregnant heifers. On day 28 , pregnancy rates were $70.4 \%$ in HF $(n=19 / 27)$, $46.7 \%$ in SF $(n=7 / 15)$ and $0 \%$ in IF $(n=0 / 4)$ heifers. Orthogonal contrasts revealed that day 28 pregnancy rate was higher $(\mathrm{P}=0.04)$ in $\mathrm{HF}$ than low fertile (SF and IF) heifers, but not different between $\mathrm{SF}$ and IF heifers $(\mathrm{P}=0.24)$. On day 36 , pregnancy rates were $63.0 \%$ in $\mathrm{HF}, 40.0 \%$ in $\mathrm{SF}$ and $0 \%$ in IF heifers. Day 36 pregnancy rates were not higher $(\mathrm{P}=$ $0.06)$ in HF than low fertile (SF and IF) heifers and not different $(\mathrm{P}=0.30)$ between $\mathrm{SF}$ and IF heifers.

Progesterone concentrations during the first 20 days post-insemination were not different $(\mathrm{P}=0.93)$ in all heifers regardless of fertility class (Fig. 2A). An effect of day (P $<0.0001)$ was detected, as progesterone concentrations increased after ovulation with formation of a corpus luteum. Further, there was an effect of day $(\mathrm{P}<0.0001)$, but not fertility class $(\mathrm{P}=0.86)$, on progesterone concentrations for $\mathrm{HF}$ and SF heifers diagnosed pregnant through day 36 (Fig. 2B). An interaction between fertility class and day of sample collection was observed $(\mathrm{P}=0.04)$, which reflected the higher $(\mathrm{P}<0.01)$ progesterone concentrations in SF than HF heifers on day 36.

\section{Pre-implantation Embryo Development in Fertility-classified Heifers (Study 3)}


Fertility-classified heifers were synchronized to estrus, and a single embryo of high quality produced in vivo was transferred into HF $(\mathrm{n}=28), \mathrm{SF}(\mathrm{n}=17)$, and IF $(\mathrm{n}=11)$ heifers on day 7 post-estrus. Conceptuses were recovered on day 14 by non-surgical uterine flush, and endometrial biopsies collected from the ipsilateral uterine horn. If recovered, conceptus length and area were determined. Embryo recovery rate was $60.7 \%$ for HF, $52.9 \%$ for SF, and $45.5 \%$ for IF heifers. Embryo donor had no effect $(\mathrm{P}=0.94)$ on embryo recovery rate. Orthogonal contrasts found that embryo recovery rate was not different $(\mathrm{P}=$ $0.48)$ in $\mathrm{HF}$ as compared to low fertility ( $\mathrm{SF}$ and IF) heifers and not different $(\mathrm{P}=0.81)$ in SF than IF heifers. Gross conceptus morphology was not different among fertilityclassifications, and a spherical to ovoid conceptus was recovered with normal morphology and obvious embryonic disc from HF, SF and IF heifers (Fig. 3A). Conceptus length was not different $(\mathrm{P}=0.47)$ in $\mathrm{HF}$ as compared to low fertility (SF and IF) heifers and not different $(\mathrm{P}=0.73)$ in $\mathrm{SF}$ as compared to IF heifers (Fig. 3B). Similarly, conceptus area was not different $(\mathrm{P}=0.82)$ between $\mathrm{HF}$ and low fertility (SF and IF) heifers and not different $(\mathrm{P}=0.73)$ between SF and IF heifers (Fig. 3B).

\section{RNA-Seq Analysis of Endometrial Biopsies (Study 3)}

After non-surgical flush on day 14 (7 days post-ET), endometrial biopsies were obtained from the ipsilateral uterine horn. Histological analysis of selected endometrial biopsies determined that the biopsy method repeatedly obtained mostly intercaruncular endometrium with glands, stroma and luminal epithelium (Fig. 4A).

Transcriptional profiling of day 14 endometrial biopsies from pregnant heifers $(\mathrm{n}=$

5 heifers per fertility class) was conducted using RNA sequencing. Sequencing of the libraries yielded more than 60 million quality reads for each sample. The reads used in data 
analysis for each sample were of minimum length $30 \mathrm{bp}$ with sliding window Phred scores of at least 30 , and were mapped to the reference genome at $96-98 \%$ across samples with $70-80 \%$ of all the pair reads mapped concordantly. Between $10 \%$ to $18 \%$ of paired reads mapped discordantly, and they likely represent reads aligning to splice or fusion sites. Less than $1 \%$ of the read pairs mapped to the genome as singletons with unmapped mates; those reads may represent repeat sequences, sequences from foreign sources, such as microbial contamination, or regions of the genome that have not been well assembled, and is not unusual [276].

A 'seed-and-vote' mapping strategy was used to quantify read counts to individual genes annotated from the reference genome[273, 274]. On average, $63 \%$ of the mapped reads were associated with at least 5 reads to individual genes in each sample. About 14\% of genes were not expressed in any samples based on absence of read counts. Such a result could be due to lack of coverage, but this is unlikely given the high coverage ( $>60$ million) of reads per sample and consistency of zero-read mapping between samples. About $0.5 \%$ of mapped reads showed mapping of 1 read on average per gene per sample. Genes were then filtered that were associated with less than or equal to 1 read per million in each library prior to fitting the count data into the generalized linear model implemented in edgeRrobust [275]. Accordingly, 26 total DEG ( $\geq 2$ fold change, FDR $\mathrm{P} \leq 0.10)$ were identified for the endometrium from HF as compared to SF heifers (Table 1). There were 12 DEGs in the endometrium from SF as compared to IF heifers (Table 2), and only 3 DEGs when HF were compared to IF heifers (Table 3).

The low number of DEGs detected by edgeR-robust analysis suggested low overall variation in gene expression across samples, which was confirmed by high pairwise correlation between samples (Fig. 4B) and lack of sample clustering as illustrated in the 
multidimensional scaling (MDS) plot (Fig. 4C). Expression values in each group were then partitioned into non-overlapping bins and plotted as conditional box plots between groups (Supplemental Fig. 1A). These plots indicate reduced gene expression changes in the endometria of IF as compared to HF or SF heifers and was not observed in HF as compared to SF heifers. The violin plots presented in Supplemental Figure 1B present distribution of $\log _{2}$ fold changes of expression relative to $\log _{2}$ counts per million reads in pair-wise manner among samples. This analysis confirmed the reduced DEG in IF as compared to HF and SF heifers.

The DEGs identified by edgeR-robust analysis of endometrial biopsy RNA sequencing are presented in Figure 4 and summarized in Tables 1,2 and 3 and Supplemental Tables 1, 2 and 3. Expression of 14 genes was higher and 12 genes lower in endometrial biopsies of HF as compared to SF heifers (Table 1 and Supplemental Table 1). Many of the 14 genes that were more abundant in the HF endometrium encoded proteins with antimicrobial activity (TAP, MUC1) or were immunoglobulins. In SF as compared to IF heifers, there were 12 genes with reduced expression in the endometrial biopsies (Table 2 and Supplemental Table 2). All 3 DEGs were lower in abundance in endometrial biopsies of HF as compared to IF heifers (Table 3 and Supplemental Table 3). Of note, 10 of the DEGs overlapped between the fertility-classified heifer endometrial biopsies.

\section{DISCUSSION}

The present study found that serial rounds of ET, each followed by pregnancy determination on days 28 and 42, is an effective strategy to identify beef heifers with high and low aggregate rates of early pregnancy loss. One might assert that by chance rates of heifers always or never being pregnant $12.5 \%$ of the time after 4 rounds of ET would be 
correct if pregnancy rate were a random binomial event; however, pregnancy is not a random binomial event and rather a cumulative physiological process in which each successive day of gestation is dependent upon the additive successful events up to that time. Similarly, McMillan and Donnison[266] utilized serial ET to classify dairy heifers as high or low fertile. Failures in mechanisms involved in conceptus elongation and maternal recognition of pregnancy were hypothesized to be the major cause of pregnancy loss in the low-fertility dairy heifers[266, 267]. Indeed, the majority of pregnancy losses in beef and dairy heifers, non-lactating cows and lactating cows occur during the first three to four weeks of pregnancy[261, 277]. Studies in sheep, beef heifers and dairy cattle established that an early or delayed rise in circulating levels of progesterone after ovulation can advance or retard conceptus elongation[250, 251, 263, 278-280]. In the present Study 2, circulating levels of progesterone were not different in fertility classified heifers, regardless of pregnancy status. This result agrees with findings from the analysis of both dairy heifers and beef heifers that were fertility classified by serial ET or AI, respectively[266, 281]. Thus, differences in circulating levels of progesterone are not a factor in the fertility differences for the beef heifers in the present study. Although increasing concentrations of progesterone after ovulation clearly advances conceptus elongation in both beef and dairy cattle, supplementation with progesterone during early pregnancy has equivocal efficacy in increasing embryonic survival[282, 283]. In fact, strategies to improve postovulatory progesterone concentration such as treatment with $\mathrm{GnRH}$ or human chorionic gonadotropin following AI have resulted in inconsistent benefits in pregnancy outcomes in lactating dairy cows[284-286]. Further, progesterone administration is unlikely to rescue development of embryos with inherent genetic defects or during gestation in highproducing dairy cows[287-289]. 
In Study 3, fertility-classified heifers received a single high quality in vivo produced embryo on day 7 post-estrus and were non-surgically flushed to recover the embryo on day 14. Embryo recovery rate and embryo morphology development (length and area) was not different among HF, SF and IF heifers. The average embryo recovery rate on day 14 was $53 \%$ across HF, SF and IF heifers, which is not much lower than the $66 \%$ pregnancy rate found on day 16 in beef heifers bred by AI [7]. The lower pregnancy rate in Study 3 could be due to a failure of the non-surgical flush procedure to recover embryos from some heifers and also the use of in vivo produced embryo that was frozen and then thawed prior to transfer. Result of the present studies support the idea that pregnancy loss in the SF and/or IF heifers must occur between days 14 and 28, which encompasses the period of conceptus elongation, pregnancy recognition and implantation for establishment of pregnancy[248, 251,263]. Given the lack of differences in conceptus survival and development, it is not surprising that substantial differences in gene expression for the endometrial biopsies from day 14 pregnant HF, SF and IF heifers were not observed. Indeed, the transcriptome difference was also minimal in other studies of fertility-classified heifers that evaluated endometria from beef heifers on day 13 post-estrus[281], endometrial cytobrush biopsies from beef heifers on day 14 post-estrus[290], or endometrial biopsies from lactating dairy cows on day 13 post-estrus[291]. The lack of conserved differences could be attributed to a myriad of factors including how fertility was classified, breed effects, and endometrial sampling. For instance, endometrium obtained at slaughter is generally a mixture of intercaruncular and caruncular endometrium, whereas the obtained biopsies in the present study are mostly intercaruncular endometrium. Of note, cytobrush sampling obtains mostly only LE from both caruncular and intercaruncular endometrium. 
Although large numbers of DEG were not identified, RNA sequencing analysis did detect differences in endometrial gene expression among the fertility-classified heifers. The majority of genes that were more abundant in HF than SF, as well as IF than SF, endometrium encode factors involved in reproductive tract defense against pathogens. For instance, TAP was first identified in the bovine tracheal mucosa and found to be a member of a group of cysteine-rich, cationic, antimicrobial peptides found in animals, insects, and plants[292]. Purified TAP had antibacterial activity in vitro against Escherichia coli, Staphylococcus aureus, Klebsiella pneumonia, and Pseudomonas aeruginosa. In addition, TAP was active against Candida albicans, indicating a broad spectrum of activity. In the present study, MUC1 was also lower in SF heifers, and it forms part of the glycocalyx barrier that provides innate immune protection against bacterial infections[293]. Additionally, MUC1 regulates implantation in mice and perhaps other mammals. Walker and coworkers[294] found evidence that the embryo modulates the uterine immune system on day 17 and induces expression of molecules in the endometrium that function to suppress the immune response and/or promote tolerance to the embryo. During this period of immune suppression, the endometrium would be expected to be susceptible to infections; therefore, it actively expresses specific molecules for defense against foreign pathogens such as upregulation of genes of the innate immune response including antimicrobial response genes like TAP. It should be noted that none of the heifers used in the present study had evidence of metritis, and endometritis is not very prevalent in beef heifers.

Many of the down-regulated genes in SF as compared to HF endometrium encoded various immunoglobulins or a transcription factor (POU2AF1) that are expressed in $\mathrm{B}$ lymphocytes, which function in the humoral immunity component of the adaptive immune 
system by secreting antibodies. Thus, B cells are likely higher in the endometrium of HF than SF heifers. Pregnancy changes the population of lymphocytes and macrophages in the bovine uterus[295-297]. The numbers of both cell types are significantly reduced between early and mid-pregnancy in cattle[298]. By mid-pregnancy, virtually no lymphocytes or macrophages are found in the caruncular endometrium although they are still present in the intercaruncular endometrium[299]. The lymphocyte population in the early pregnant cattle uterus is composed primarily of B cells, T cells and natural killer cells[300-302]. B lymphocytes are widely distributed throughout the endometrium, localizing in the stroma, myometrium, and the luminal and glandular epithelium. The B lymphocyte population in bovine endometrium was relatively large compared to the populations of $\gamma \delta \mathrm{T}, \mathrm{CD} 4+$, CD8+ and NK cells detected[300]. Although several genes were more abundant in the endometrium of SF than HF and SF than IF heifers, the function of most of those genes is unknown. Collectively, these results support the idea that the innate and adaptive immune system is different in the endometrium of SF as compared to that of HF and IF heifers, which could be involved in the observed fertility differences[297]. The lack of conserved differences in the endometrial transcriptome of the HF versus low fertility (SF and IF) heifers indicates that the biological mechanisms underlying subfertility and infertility are possibly different, and those differences may not manifest until after day 14 as the conceptus begins rapid growth for elongation. Future experiments will need to explore differences in the endometrial secrotome, because histotroph has a major influence on growth and development of the conceptus[245, 251, 263].

The present study and others[266, 267, 281, 290,291] support the hypothesis that natural variation in pregnancy rates can be utilized in cattle to identify animals with innate differences in uterine competence to support growth and development of the conceptus for 
establishment of pregnancy. Although not addressed in the present study, it is also important to address the substantial loss of embryos that occurs prior to day 7 , particularly in lactating dairy cows, due to problems with oocyte competence, sperm transport, fertilization and perhaps oviductal-uterine function[303, 304]. Studies of animals with natural variation in uterine competency for pregnancy could help define which genes and biological pathways in the endometrium are crucial to establish endometrial receptivity and support conceptus elongation in cattle. Further, the use of this animal model could discover genes and biomarkers that can be used to select animals for higher fertility and to diagnose subfertility and infertility.

\section{ACKNOWLEDGMENTS}

The authors appreciate the assistance of staff at the USDA-ARS Fort Keogh with animal handling and sample collection, William Rembert for collecting ovaries, the Chernin family and Central Packing (Center Hill, Florida) for donating ovarian tissue, and Scott Randel of Southeastern Semen (Wellborn Florida) for donating semen, and Zoetis for donation of synchronization products. They also appreciate the help of Drs. Mike Smith and Duane Keisler and the MU DNA Core facility of the University of Missouri-Columbia with completion of the studies. 


\section{FIGURES}
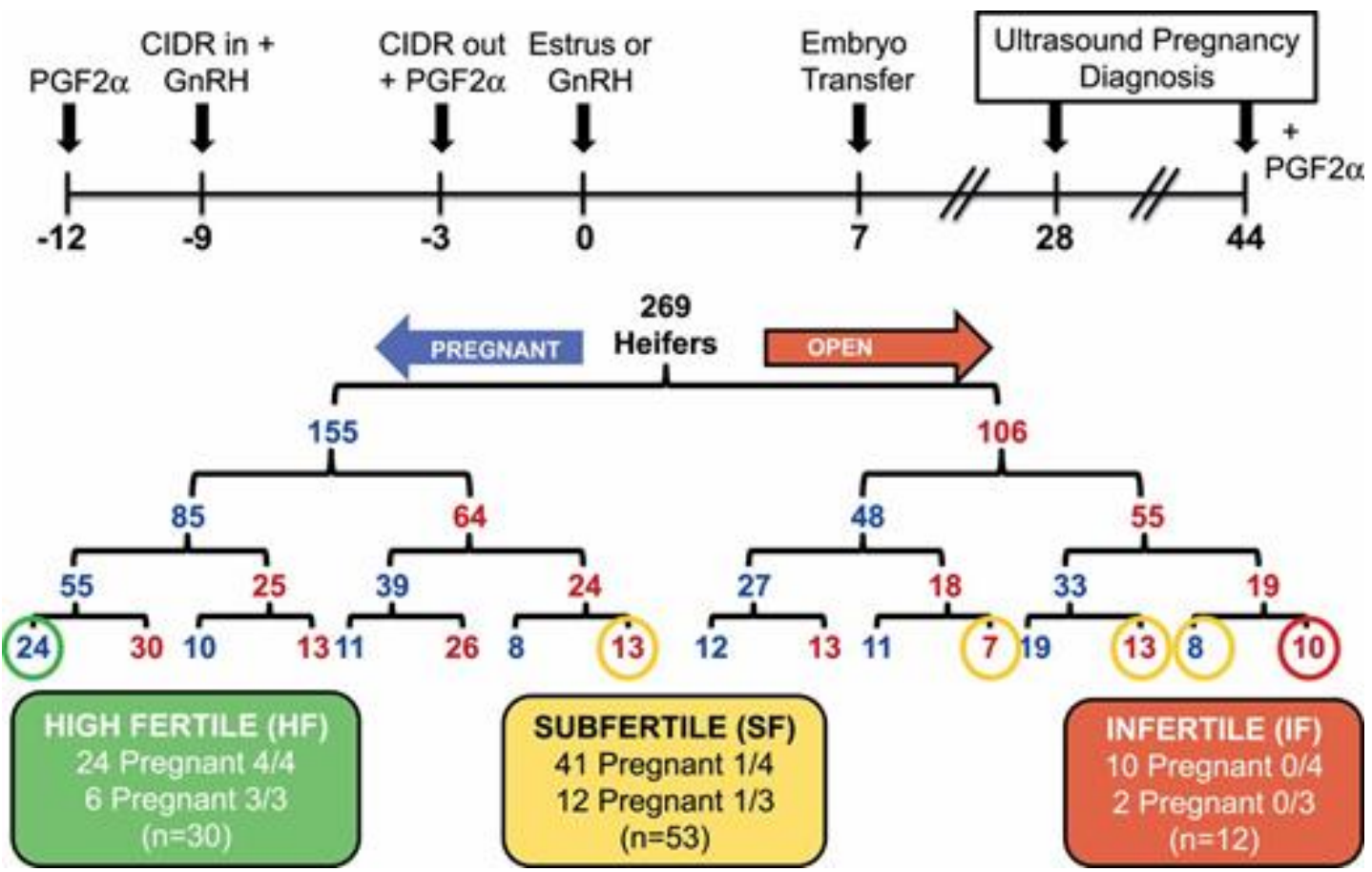

Figure 1. Experimental design and results for classification of fertility in beef heifers using serial embryo transfer (ET).

See text for detailed description of results. Each row of numbers will not add up to 269 because of occasional death loss or removal of heifers from the study for health reasons. Further, 37 heifers did not have a CL at least once, and 5 heifers did not have a CL on 2 occasions. Abbrevations: CIDR, controlled intravaginal drug releasing device; GnRH, gonadotropin releasing hormone; PGF2, prostaglandin F2 alpha. 
A

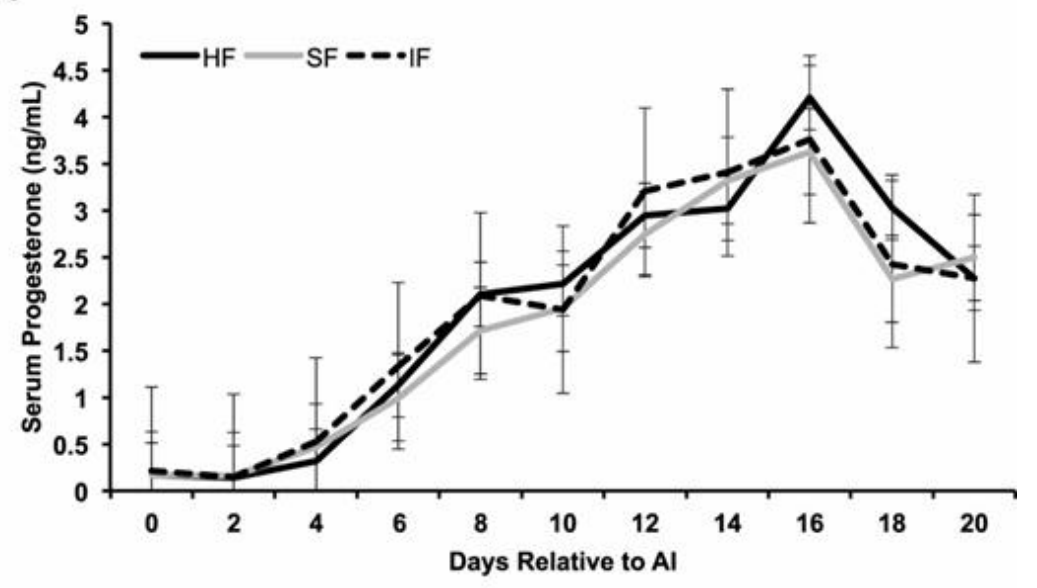

B

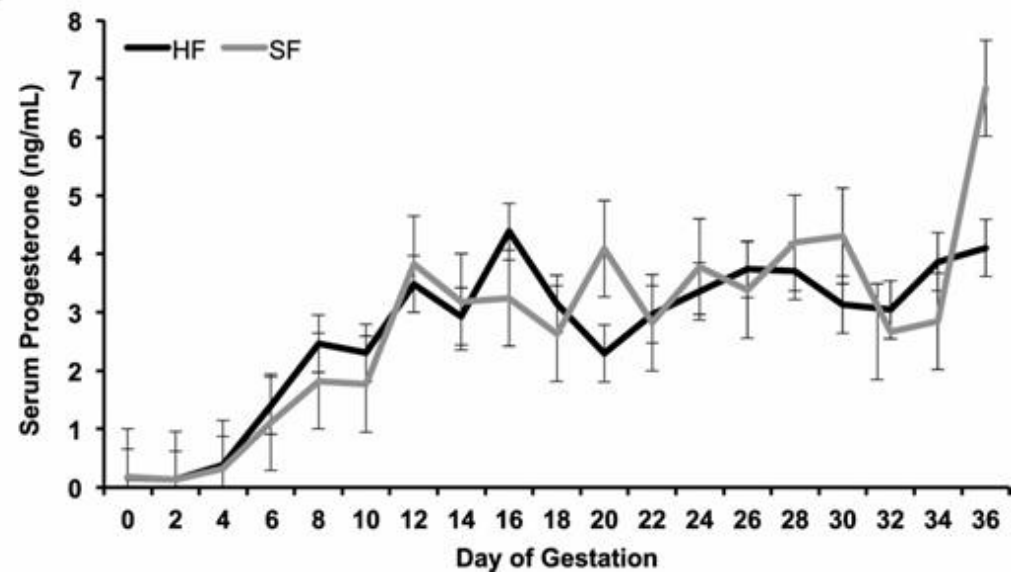

Figure 2. Circulating concentrations of progesterone in nonpregnant (A) and pregnant (B) fertility classified heifers.

Fertility classified heifers were synchronized to estrus and bred by artificial insemination (AI) at $12 \mathrm{~h}$ and $24 \mathrm{~h}$ post-estrus with semen from a high fertility bull. Pregnancy was determined on days 28 and 35 by ultrasound. An effect of day $(\mathrm{P}<0.0001)$ was detected, as progesterone concentrations increased after ovulation. There was an effect of day $(\mathrm{P}<$ $0.0001)$, but not fertility class $(\mathrm{P}=0.86)$, on progesterone concentrations for $\mathrm{HF}$ and $\mathrm{SF}$ heifers diagnosed pregnant through day 36. Progesterone concentrations were higher in SF than HF heifers on day $36(\mathrm{P}=0.04$, day $\mathrm{x}$ fertility class $)$. Data is presented as least squares means (LSM) with standard error (SE). 
A
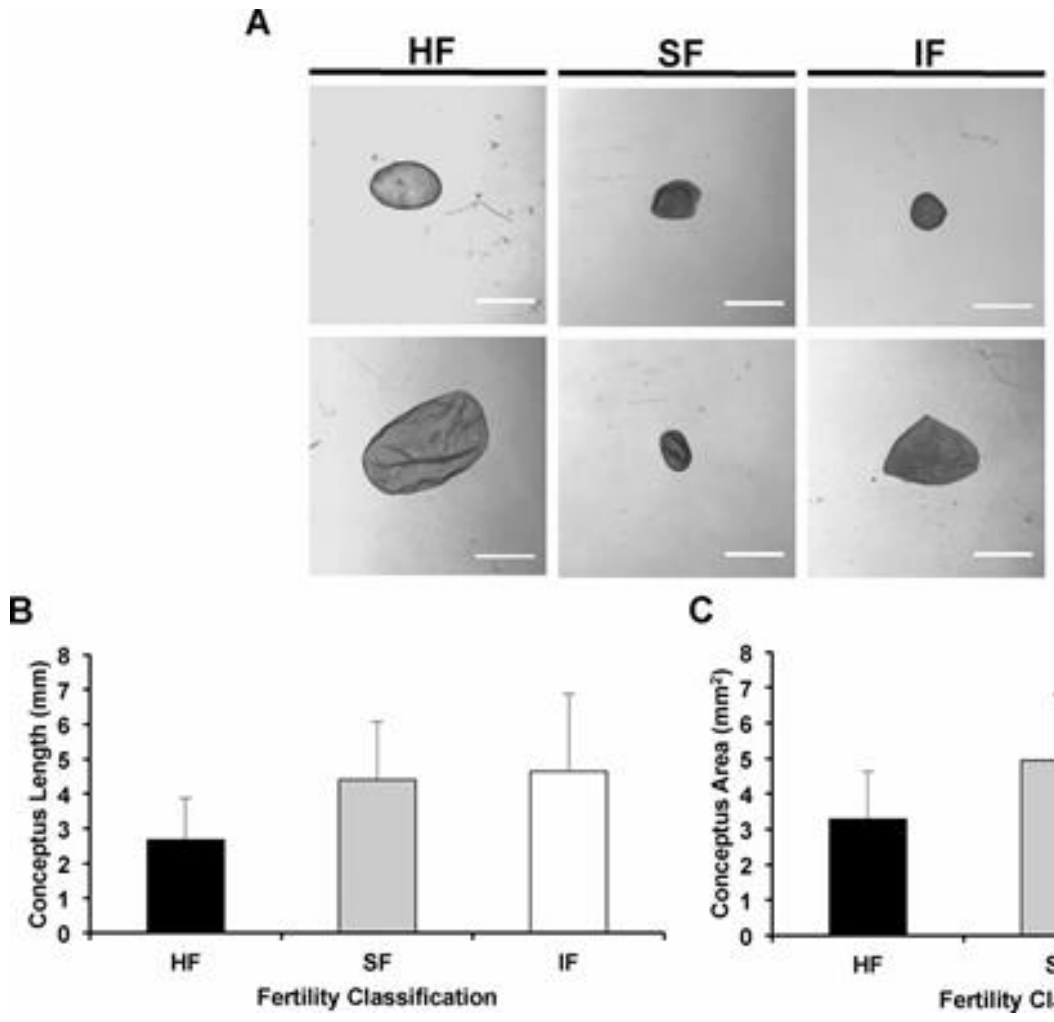

C

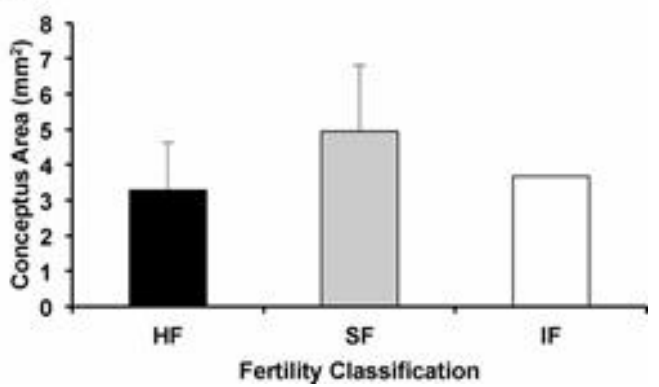

Figure 3. Day 14 conceptus morphology and measurements.

Fertility classified heifers were synchronized to estrus and received a high quality in vivo produced embryo on day 7 post-estrus. All heifers were nonsurgically flushed on day 14 (7 days post-ET) to recover the conceptus. If present, conceptus length and width were determined under a microscope. (A) Representative conceptus morphology recovered from high fertile (HF), subfertile (SF) and infertile (IF) heifers. Scale bar $=1 \mathrm{~mm}$. (B and $\mathbf{C})$ Conceptus length and area were not different $(\mathrm{P} \geq 0.60)$ among the fertility classified heifers. Data is presented as least squares means (LSM) with standard error (SE). 
A

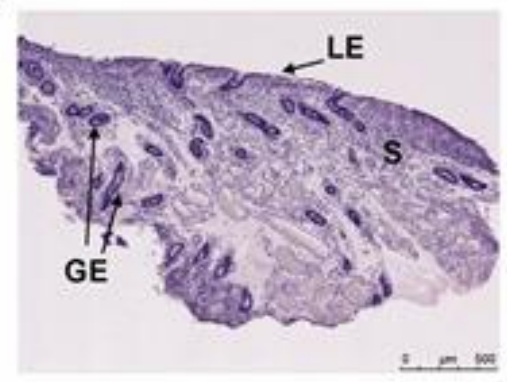

\section{B}

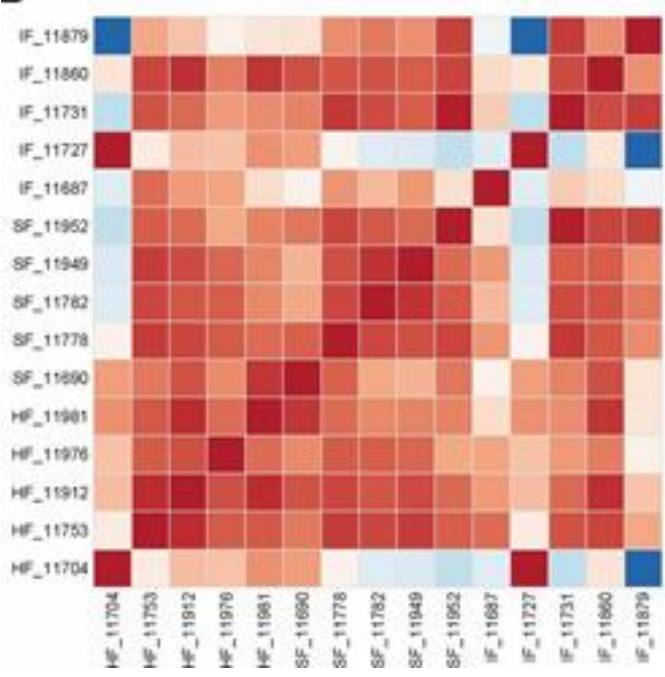

C

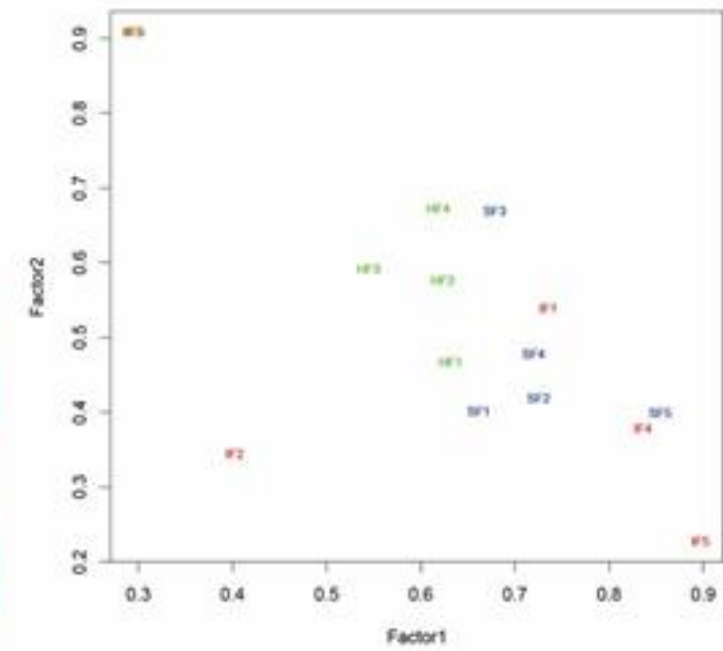

Figure 4. Endometrial biopsy histology and RNA sequencing analysis from day 14 pregnant heifers.

Fertility classified heifers were synchronized to estrus and received two high quality in vivo produced embryos on day 7 post-estrus. All heifers were nonsurgically flushed on day 14 (7 days post-ET) to recover the conceptus. If a conceptus was present in the uterine flush, an endometrial biopsy was obtained from the uterine horn ipsilateral to the corpus luteum. Total RNA was extracted from 5 biopsies of pregnant high fertile (HF), subfertile (SF) and infertile (IF) heifers and sequenced. Normalized and $\log _{2}$ transformed read count data were produced with edgeR-robust analysis. (A) Histological analysis of a representative endometrial biopsy. All biopsies were predominantly composed of intercaruncular endometrium. Sections were stained with hematoxylin and eosin. LE, 
luminal epithelium; GE, glandular epithelium; S, stroma. Scale bar $=500 \mu \mathrm{m}$. (B) Pairwise correlation (Pearson) analysis of gene expression levels between endometrial biopsy samples. Each column represents one sample and shows the correlation to all samples (including itself) with red for lowest (0) distance and blue for the highest observed distance. (C) Multidimensional scaling (MDS) plot. A maximum likelihood factor plot of gene expression variation among the samples. The major factors (factor 1 and factor 2) that explain the expression changes among the samples are plotted in $\mathrm{x}$ - and $\mathrm{y}$-axis respectively. The individual samples $(\mathrm{n}=15)$ representing the three fertility groups $(\mathrm{HF}, \mathrm{SF}$ and IF) are shown with different colors (green, blue and red, respectively). 


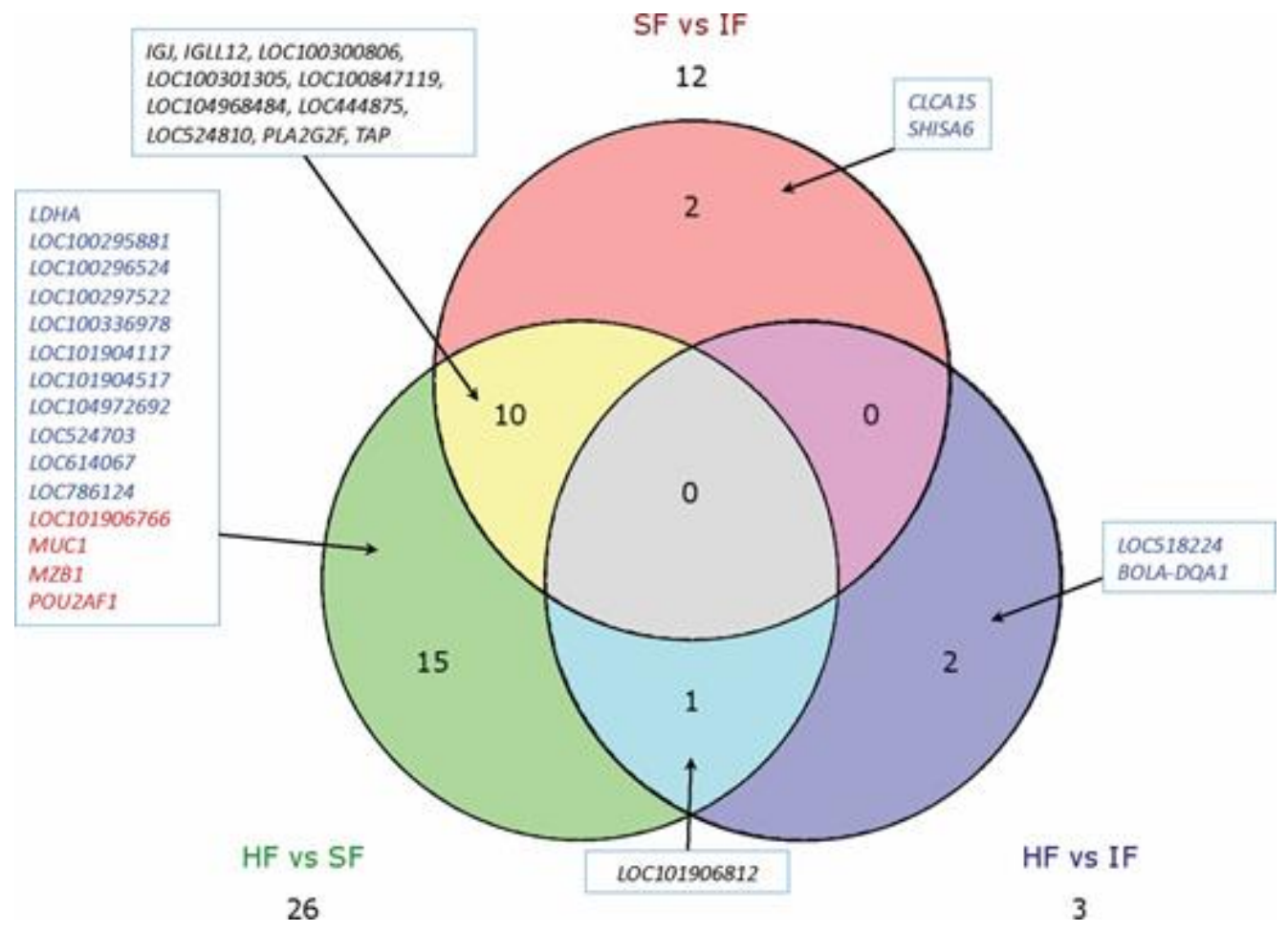

Figure 5. Venn diagram for differently expressed genes in the endometrium of pregnant fertility-classified heifers.

Venn diagram showing the number of unique or common transcripts between the endometrium of fertility-classified heifers (HF, high fertile; SF, subfertile; IF, infertile). Increased (red) and decreased (blue) genes are presented $(\geq 2$ fold change and FDR $\mathrm{P} \leq$ $0.05)$. 


\section{TABLES}

Table 1. Differentially expressed genes in endometrial biopsies from high fertile (HF) and subfertile (SF) heifers

\begin{tabular}{|c|c|c|c|c|c|}
\hline \multirow[t]{2}{*}{ Gene symbol } & \multirow[t]{2}{*}{ Gene name ${ }^{a}$} & \multirow[t]{2}{*}{$\log \mathrm{FC}^{\mathrm{b}}$} & \multirow[t]{2}{*}{ FDR $^{c}$ P-value } & \multicolumn{2}{|c|}{ Mean FPKM ${ }^{\mathrm{d}}$} \\
\hline & & & & HF & SF \\
\hline TAP & Tracheal antimicrobial peptide & 7.25 & 0.01 & 7.36 & 0.00 \\
\hline LOC100847119 & Ig lambda-7 chain $\mathrm{C}$ region-like & 5.93 & 0.00 & 66.29 & 0.95 \\
\hline LOC524810 & $\operatorname{IgM}$ & 5.86 & 0.00 & 220.13 & 3.79 \\
\hline LOC100301305 & Ig heavy chain V region MC101-like & 4.49 & 0.00 & 10.84 & 0.41 \\
\hline LOC100300806 & Ig heavy chain Mem5-like & 4.08 & 0.00 & 53.94 & 3.18 \\
\hline IGLL1-2 & Unknown & 3.96 & 0.00 & 232.32 & 14.97 \\
\hline LOC104968484 & Ig heavy chain Mem5-like & 3.90 & 0.00 & 123.90 & 8.38 \\
\hline PLA2G2F & Phospholipase A2 group IIF & 3.84 & 0.04 & 0.91 & 0.07 \\
\hline LOC444875 & Ig heavy chain $\mathrm{V}$ region PJ14 & 3.66 & 0.00 & 66.72 & 5.31 \\
\hline LOC101906766 & Ig lambda chain V-II region VIL-like & 3.54 & 0.00 & 5.55 & 0.47 \\
\hline IGJ & Immunoglobulin joining chain & 3.48 & 0.00 & 112.72 & 10.13 \\
\hline POU2AF1 & POU class 2 associating factor 1 & 2.70 & 0.03 & 0.29 & 0.04 \\
\hline MZB1 & Marginal zone B and B1 cell-specific protein & 1.85 & 0.05 & 2.87 & 0.80 \\
\hline MUC1 & Mucin 1, cell surface associated & 1.00 & 0.05 & 55.64 & 27.30 \\
\hline LDHA & Lactate dehydrogenase A & -1.02 & 0.05 & 18.09 & 36.58 \\
\hline LOC 101904117 & Uncharacterized LOC101904117 & -2.28 & 0.01 & 125.54 & 591.83 \\
\hline LOC614067 & Ribosomal protein L21 pseudogene & -2.59 & 0.03 & 14.60 & 88.13 \\
\hline LOC104972692 & Uncharacterized LOC104972692 & -3.59 & 0.05 & 0.45 & 5.53 \\
\hline LOC524703 & 40S ribosomal protein $\mathrm{S} 21$ pseudogene & -3.66 & 0.04 & 0.52 & 6.62 \\
\hline LOC 100336978 & Multidrug resistance-associated protein 4 & -3.76 & 0.03 & 0.46 & 5.55 \\
\hline LOC100297522 & $60 \mathrm{~S}$ ribosomal protein $\mathrm{L} 38$ & -3.77 & 0.02 & 2.72 & 37.20 \\
\hline LOC786124 & Prostaglandin E synthase 3 pseudogene & -4.04 & 0.03 & 0.80 & 13.27 \\
\hline LOC 101904517 & Multidrug resistance-associated protein 4-like & -4.22 & 0.00 & 0.08 & 0.69 \\
\hline LOC100296524 & 60S ribosomal protein L39 & -5.14 & 0.04 & 0.36 & 12.94 \\
\hline LOC101906812 & Uncharacterized LOC101906812 & -5.20 & 0.02 & 0.47 & 2.65 \\
\hline LOC100295881 & Ribosomal protein S27-like pseudogene & -5.36 & 0.01 & 2.38 & 98.15 \\
\hline
\end{tabular}

${ }^{\text {a }}$ Ig, immunoglobulin.

${ }^{\mathrm{b}} \log 2$ fold change $(\log \mathrm{FC})$ in $\mathrm{HF}$ as compared to SF endometrial biopsy samples.

${ }^{\mathrm{c}} \mathrm{FDR}$, false discovery rate.

${ }^{\mathrm{d}}$ Data are presented as mean fragments per kilobase of exon per million (FPKM) mapped reads. 
Table 2. Differentially expressed genes in endometrial biopsies from subfertile (SF) and infertile (IF) heifers

\begin{tabular}{|c|c|c|c|c|c|}
\hline \multirow[t]{2}{*}{ Gene symbol } & \multirow[t]{2}{*}{ Gene name $^{a}$} & \multirow[t]{2}{*}{$\underset{b}{\log F C}$} & \multirow[t]{2}{*}{$\begin{array}{l}\text { FDR }^{\mathrm{c}} \mathrm{P}- \\
\text { value }\end{array}$} & \multicolumn{2}{|c|}{$\begin{array}{c}\text { Mean } \\
\text { FPKM }^{\text {d }} \\
\end{array}$} \\
\hline & & & & $\begin{array}{l}\text { SF } \\
10.1\end{array}$ & IF \\
\hline $\begin{array}{l}\text { IGJ } \\
\text { LOC10496848 }\end{array}$ & Immunoglobulin joining chain & -2.22 & 0.01 & & 72.1 \\
\hline 4 & Ig heavy chain Mem5-like & -2.58 & 0.00 & $\begin{array}{l}8.4 \\
15.0\end{array}$ & 57.0 \\
\hline IGLL1-2 & Unknown & -2.61 & 0.00 & & 106.1 \\
\hline $\begin{array}{l}\text { LOC } 444875 \\
\text { LOC } 10030080\end{array}$ & Ig heavy chain V region PJ14 & -2.68 & 0.00 & 5.3 & 34.5 \\
\hline LOC10030130 & $\begin{array}{l}\text { Ig heavy chain Mem5-like } \\
\text { Ig heavy chain V region MC101- }\end{array}$ & -2.71 & 0.00 & 3.2 & 26.2 \\
\hline $\begin{array}{l}5 \\
\text { LOC10084711 }\end{array}$ & like & -3.18 & 0.00 & 0.4 & 4.3 \\
\hline 9 & Ig lambda-7 chain $\mathrm{C}$ region-like & -3.26 & 0.00 & 1.0 & 11.1 \\
\hline CLCA1 & Chloride channel accessory 1 & -3.59 & 0.00 & 0.1 & 1.5 \\
\hline LOC524810 & $\operatorname{IgM}$ & -4.64 & 0.00 & 3.8 & 115.4 \\
\hline PLA2G2F & Phospholipase A2 group IIF & -4.85 & 0.00 & 0.1 & 1.5 \\
\hline SHISA6 & Shisa family member 6 & -5.15 & 0.02 & 0.0 & 0.2 \\
\hline TAP & Tracheal antimicrobial peptide & -8.15 & 0.00 & 0.0 & 7.9 \\
\hline
\end{tabular}

${ }^{\text {a }}$ Ig, immunoglobulin.

${ }^{\mathrm{b}} \mathrm{Log} 2$ fold change $(\log \mathrm{FC})$ in SF as compared to IF endometrial biopsy samples.

${ }^{c}$ FDR, false discovery rate.

${ }^{\mathrm{d}}$ Data are presented as mean fragments per kilobase of exon per million (FPKM) mapped reads. 
Table 3. Differentially expressed genes in endometrial biopsies from high fertile (HF) and infertile (IF) heifers

\begin{tabular}{|c|c|c|c|c|c|}
\hline \multirow[t]{2}{*}{ Gene symbol } & \multirow[t]{2}{*}{ Gene name } & \multirow[t]{2}{*}{$\begin{array}{c}\operatorname{LogFC} \\
a \\
\end{array}$} & \multirow[t]{2}{*}{$\begin{array}{l}\text { FDR P- } \\
\text { value }\end{array}$} & \multicolumn{2}{|c|}{$\begin{array}{c}\text { Mean } \\
\text { FPKMb }\end{array}$} \\
\hline & & & & HF & IF \\
\hline \multirow[t]{2}{*}{ LOC518224 } & Membrane cofactor protein & -4.01 & 0.05 & 0.2 & 0.9 \\
\hline & Major histocompatibility & & & & \\
\hline \multirow{3}{*}{$\begin{array}{l}\text { BOLA-DQA1 } \\
\text { LOC101906812 }\end{array}$} & $\begin{array}{l}\text { complex, class II, DQ alpha, } \\
\text { type } 1\end{array}$ & -4.67 & 0.02 & 0.6 & 19.0 \\
\hline & Uncharacterized & & & & \\
\hline & LOC101906812 & -5.15 & 0.02 & 0.0 & 2.4 \\
\hline
\end{tabular}

${ }^{a} \log 2$ fold change $(\log \mathrm{FC})$ in $\mathrm{HF}$ as compared to IF endometrial biopsy samples.

${ }^{b}$ Data are presented as mean fragments per kilobase of exon per million (FPKM) mapped reads. 


\section{SECTION III:}

\section{Uterine Influences on Conceptus Development in Fertility-Classified Animals}

Joao G.N. Moraes, Susanta K. Behura, Thomas W. Geary, Peter J. Hansen, Holly L. Neibergs, and Thomas E. Spencer

This work has been published in:

PNAS February 20, 2018115 (8) E1749-E1758; doi: 10.1073/pnas.1721191115 


\begin{abstract}
A major unresolved issue is how the uterus influences infertility and subfertility in cattle. Serial embryo transfer was previously used to classify heifers as high fertile (HF), subfertile (SF), or infertile (IF). To assess pregnancy loss in those animals, two in vivo produced embryos were transferred into HF, SF and IF heifers on day 7, and pregnancy outcome assessed on day 17. Pregnancy rate was substantially higher in HF (71\%) and SF $(90 \%)$ than IF $(20 \%)$ heifers. Elongating conceptuses were about two-fold longer in HF than SF heifers. Transcriptional profiling detected relatively few differences in endometrium of nonpregnant HF, SF and IF heifers. In contrast, there was a substantial difference in the transcriptome response of the endometrium to pregnancy between HF and SF heifers. Considerable deficiencies in pregnancy-dependent biological pathways associated with extracellular matrix structure and organization as well as cell adhesion were found in endometrium of SF animals. Distinct gene expression differences were also observed in conceptuses from HF and SF animals with many of the genes decreased in SF conceptuses known to be embryonic lethal in mice due to defects in embryo and/or placental development. Analyses of biological pathways, key players, and ligand-receptor interactions based on transcriptome data divulged substantial evidence for dysregulation of conceptus-endometrial interactions in SF animals. These results support the ideas that: the uterus impacts conceptus survival and programs conceptus development; and ripple effects of dysregulated conceptus-endometrial interactions elicit loss of the post-elongation conceptus in SF cattle during the implantation period of pregnancy.
\end{abstract}




\section{INTRODUCTION}

Infertility and subfertility are important and pervasive problems in agricultural animals and humans $[305,306]$. In ruminants, embryo mortality is a major factor affecting fertility and thus production and economic efficiency $[2,15]$. Pregnancy loss in cattle ranges from $30 \%$ to $56 \%$ with the majority of losses occurring in the first month of pregnancy [2, 36, 307]. Infertility and subfertility also impact the success of embryo transfer (embryo transfer) in cattle and humans [308, 309]. In cattle, mean survival rate to calving following transfer of in vivo-derived embryos from superovulated donors ranges from $31 \%$ to $60 \%$, and in vitro-produced embryo survival rate is lower [36, 310]. Failure

of the embryo to survive and establish pregnancy is due to paternal, maternal and embryonic factors [311-313]. Many of the pregnancy losses observed in natural or assisted pregnancies may be attributed to maternal factors such as an inability of the uterus to support conceptus growth and implantation [314, 315].

After fertilization (day 0), the zona pellucida-enclosed bovine embryo enters the uterus at the morula stage by day 5 and develops into a blastocyst. The spherical blastocyst hatches from the zona pellucida on days 7 to 10 and continues to grow, changing from spherical to ovoid in shape between days 12 and 14 after which it can be termed a conceptus (embryo and associated extra-embryonic membranes) [316]. The conceptus undergoes elongation involving exponential growth from about $2 \mathrm{~mm}$ in length on day 13, $60 \mathrm{~mm}$ by day 16 , and $20 \mathrm{~cm}$ or more by day 19 [95]. After days 16-17, the time of maternal recognition of pregnancy (when the embryo signals to the mother to prevent regression of the corpus luteum), the conceptus begins the processes of implantation and placentation that involves apposition, attachment and adhesion of the trophectoderm to the endometrial luminal epithelium and onset of trophoblast giant binucleate cell differentiation [99]. 
Blastocyst growth into an elongated conceptus has not been achieved in vitro and requires transfer into the uterus [97], as the endometrium secretes or transports a myriad of factors critical for conceptus growth and elongation $[110,315]$. Dynamic changes in the endometrial transcriptome occur between days 7 and 13 that are primarily regulated by progesterone in both nonpregnant and pregnant cattle [110, 253, 315, 317]. Those changes in the endometrium presumably establish a uterine environment conducive to blastocyst survival and conceptus growth into an elongated, filamentous type conceptus and subsequently implantation and placentation. Conceptus elongation is required for production of interferon tau (IFNT) [318], which is the pregnancy recognition signal that acts on the endometrium to sustain continued production of progesterone by the ovary and regulates genes implicated in conceptus growth, implantation and placentation $[319,320]$. Inadequate elongation of the conceptus presumably results in lower production of IFNT, inability to maintain the corpus luteum, and thus pregnancy loss [320-322]. Although much information is known about embryo development into a blastocyst from in vitro systems [323], the essential endometrial genes and biological pathways important for conceptus survival, growth and implantation remain largely unknown [324, 325].

One of the major impediments to understanding maternal influences on pregnancy loss has been a lack of animals with defined high and low rates of early pregnancy loss. McMillan and Donnison [326] summarized a unique approach for experimentally identifying high and low fertility in dairy heifers based on serial transfer of in vitroproduced embryos. Their approach identified animals with high (76\%) and low (11\%) aggregate pregnancy rates, and a failure in the mechanism involved in conceptus elongation and thus maternal recognition of pregnancy was suggested to be the cause of early pregnancy loss in the low fertility-classified heifers [326, 327]. We recently used a similar 
approach to identify beef heifers with intrinsic differences in pregnancy loss [328]. Serial transfer of a single in vitro-produced embryo was used to classify animals as high fertile (HF), subfertile (SF), or infertile (IF) based on day 28 pregnancy rate. In those heifers, no difference in pregnancy rates were observed on day 14 after transfer of a single in vivoproduced embryo on day 7 post-estrus [328]. Thus, the observed difference in uterine competence for pregnancy in fertility-classified heifers is hypothesized to manifest during maternal recognition of pregnancy and implantation. The present study tested this hypothesis and revealed that embryo survival to day 17 is compromised in IF heifers and that asynchronous conceptus-endometrial interactions in SF animals leads to postelongation embryonic loss by day 28 during the implantation period of pregnancy.

\section{MATERIALS AND METHODS}

Animals. All animal procedures were conducted in accordance with the Guide for the Care and Use of Agriculture Animals in Research and Teaching and approved by the Institutional Animal Care and Use Committees of the USDA-ARS Fort Keogh Livestock and Range Research Laboratory and the University of Missouri. In our recent work [328], 269 beef heifers were classified based on fertility using serial embryo transfer to select animals with intrinsic differences in pregnancy loss. In each of the four rounds, a single in vitro-produced high-quality embryo was transferred into heifers on day 7 post-estrus and pregnancy was determined on days 28 and 42 by ultrasound and then terminated. Heifers were classified based on pregnancy success as high fertile $(\mathrm{HF}=100 \%)$, subfertile ( $\mathrm{SF}=25$ $33 \%)$, or infertile $(\mathrm{IF}=0 \%)$. In the present experiment, 41 heifers that have been previously [328] classified for fertility (HF, $n=21 ; \mathrm{SF}, \mathrm{n}=14 ; \mathrm{IF}, \mathrm{n}=6$ ) were used. 
Embryos. In vivo produced embryos were generated at the USDA-ARS, Fort Keogh Livestock and Range Research Station using 7 Angus donor cows using previously described methods [328] and a total amount of FSH equivalent to $320 \mathrm{mg}$ NIH-FSH-P1 (Folltropin-V, Vetoquinol, Quebec, Canada). Donors cows were inseminated $12 \mathrm{~h}$ and 24 $\mathrm{h}$ after onset of estrus (day 0 and 0.5 ) with semen from a single high fertile Holstein sire, and were flushed on day 7 after breeding for embryo recovery. Recovered embryos were classified by stage of development and graded based on morphological appearance (Manual of the International Embryo Transfer Society, 4th edition). Two quality grade 1 or 2 embryos (a compact morula and a blastocyst) were loaded into a $0.25 \mathrm{cc}$ polyvinyl straw containing ViGro Freeze Plus medium (Bioniche Animal Health, Belleville, Ontario) and frozen in a programmable embryo freezer using standard techniques for direct transfer [390].

Embryo transfer and Sample Collection. Estrous cycles of fertility-classified heifers were synchronized with an injection of PGF2 $\alpha$ followed in 3 days by an injection of GnRH concurrent with the insertion of a CIDR insert. Six days later, CIDR inserts were removed and an injection of PGF2 $\alpha$ was administered. Concomitant with CIDR removal, estrus detection patches (Estrotect, Rockway, Inc., Spring Valley, WI) were applied on the tail head of each heifer to aid in visual detection of estrus. Heifers were observed for signs of estrus 3 times a day, beginning at $24 \mathrm{~h}$ after CIDR removal. On day 7 post-estrus, each heifer received two in vivo-produced embryos placed in the uterine horn ipsilateral to the ovary containing a corpus luteum using standard non-surgical techniques by a single technician. Ultrasonography was used to identify side and presence of the corpus luteum before embryo transfer. Only heifers that exhibited estrus signs after CIDR removal and 
PGF2 $\alpha$ injection and that had a corpus luteum on day 7 post-estrus received embryos. For collection of nonpregnant animals, heifers were synchronized to estrus (day 0) but did not receive embryos on day 7.

The female reproductive tract was recovered on day 17 post-estrus, and their uteri were flushed with $20 \mathrm{~mL}$ of filtered sterile PBS (pH 7.2). If present, the state of conceptus development was assessed using a Nikon SMZ1000 stereomicroscope (Nikon Instruments Inc.) fitted with a Nikon DS-Fil digital camera. Conceptus size was determined using a ruler. The volume of the uterine flush was measured, and the flush clarified by centrifugation $\left(3,000 \times \mathrm{g}\right.$ at $4^{\circ} \mathrm{C}$ for $\left.15 \mathrm{~min}\right)$. The supernatant was carefully removed with a pipette, mixed, aliquoted, and stored at $-80^{\circ} \mathrm{C}$ until analyzed. The endometrium was physically dissected from the remainder uterine horn using curved scissors. Endometrial samples as well as conceptuses were frozen in liquid nitrogen and stored at $-80^{\circ} \mathrm{C}$ for subsequent RNA extraction.

Circulating Progesterone Concentrations. For determination of circulating progesterone concentrations on the day of slaughter (day 17 post-estrus), blood samples were collected from the median coccygeal vein or artery into evacuated tubes containing K3 EDTA (Becton Dickinson Vacutainer Systems, Franklin Lakes, NJ, USA). Blood tubes were then centrifuged at $1,200 \mathrm{x}$ g for $20 \mathrm{~min}$ at $4{ }^{\circ} \mathrm{C}$ and plasma collected and stored at $-20^{\circ} \mathrm{C}$ until a radioimmunoassay was performed. Plasma concentrations of progesterone were determined in duplicate $100 \mu \mathrm{l}$ aliquots of sample using manufacturer (MP Biomedical, Santa Ana, CA) reagents and recommendations for the liquid-liquid phase double antibody 
precipitation assay (MP Biomedicals cat\# 07-170105), and validated as previously described (112). Intra-assay coefficient of variation was $2 \%$.

Statistical analysis. Statistical analyses were conducted using SAS (SAS Institute Inc., Cary, NC). Statistical significance was defined as $\mathrm{P} \leq 0.05$. Binomial data representing whether or not a conceptus was recovered on day 17 uterine flush (Yes $=$ at least one conceptus was recovered; No = no conceptus was recovered) was analyzed by logistic regression with Firth's bias correction using the LOGISTIC procedure. The proportion of heifers that had at least one conceptus recovered on day 17 flush within each fertility classification was determined using FREQ procedure. The effect of fertility classification on the number of conceptuses recovered was analyzed by logistic regression using the GLIMMIX procedure with a multinomial distribution. Post-test comparisons were conducted using the contrast statement. The effect of fertility classification on conceptus length was estimated in a Poisson regression repeated measurements model using GENMOD procedure to account for the effect of more than one conceptus being recovered per heifer. The means of conceptus length were estimated using the MEANS procedure. Continuous variables were assessed for normality using the UNIVARIATE procedure. The effect of pregnancy status (pregnant vs nonpregnant) and fertility classification on plasma progesterone concentrations was determined by ANOVA using the GLM procedure. Posttest comparisons were conducted using the LSMEANS statement with the Fisher's protected LSD option. Only heifers which only one conceptus was recovered were used in the analysis to investigate the correlation of plasma progesterone concentration and conceptus size. Pearson's correlations were determined using the CORR procedure. 
RNA Sequencing. Total RNA from day 17 endometrium samples of 25 heifers- HF nonpregnant $(\mathrm{n}=5)$, HF pregnant $(\mathrm{n}=5), \mathrm{SF}$ nonpregnant $(\mathrm{n}=5)$, SF pregnant $(\mathrm{n}=5$, and IF nonpregnant $(\mathrm{n}=5)$ was extracted using the Isol-RNA Lysis Reagent (5Prime, Hamburg, Germany). Briefly, frozen endometrium samples were disrupted and homogenized in Isol-RNA Lysis Reagent with the use of a homogenizer (VDI 25, VWR International, Radnor, PA) and total RNA purified following the manufacturer's instructions. Total RNA from 27 conceptuses (HF, $\mathrm{n}=17$; $\mathrm{SF}, \mathrm{n}=10$ ) was extracted using the AllPrep DNA/RNA/Protein Mini kit (Qiagen, Valencia, CA).

To eliminate DNA contamination, total RNA from endometrium and conceptuses was treated with DNase I during RNA purification using the RNase-Free DNase Set (Qiagen, Valencia, CA). RNA concentration was determined by quantitative high sensitivity RNA analysis on the Fragment Analyzer instrument (Catalog \# DNF-472, Advanced Analytical Technologies, Inc., Ankeny, IA). RNA library preparation and sequencing was conducted by the University of Missouri DNA Core facility. Libraries were constructed following the manufacturer's protocol with reagents supplied in Illumina's TruSeq mRNA stranded sample preparation kit. Briefly, the polyadenylated mRNA was purified from total RNA and fragmented. Double-stranded cDNA was generated from fragmented RNA, and the index containing adapters ligated. The final construct of each purified library was evaluated using the Fragment Analyzer instrument, quantified with the Qubit fluorimeter using the quant-iT HS dsDNA reagent kit (Invitrogen), and diluted according to Illumina's standard sequencing protocol for sequencing on an Illumina NextSeq 500 sequencer. All the raw data and processed data from this study have been submitted to the Gene Expression Omnibus for public access (accession no. GSE107891). 
Alignment of Sequences and Analyses of Differential Gene Expression. The raw sequences (fastq) were subjected to adapter removal and quality trimming using fqtrim (https://ccb.jhu.edu/software/fqtrim/). The quality reads were then mapped to the bovine reference genome UMD3.1 using Hisat2 mapper (https://ccb.jhu.edu/software/hisat2/) which is a fast and sensitive alignment program of next-generation sequencing data [391]. To produce a global gene annotation file (GTF) for comprehensive quantification of gene expressions across the samples, the sorted binary alignment maps of sequence reads from each sample were assembled to generate transcriptomes those were then merged, along with the gene annotations from reference genome assembly. The transcript assembly step was accomplished using the software 'Stringtie' (https://ccb.jhu.edu/software/stringtie/) which is considered as a highly efficient assembler of RNA-Seq mapping data [392]. The merged global transcriptome was then used in Stringtie to quantify transcript abundance of each sample. Differential expression analysis between sample groups were performed by robustly fitting the expression data to a weighted generalized linear model using edgeR robust [393].

GO, Pathway and Network Analysis. Additional analyses determined if DEGs were significantly enriched in specific pathways and expression networks. First, a model-based cluster analysis of differential gene expressions was performed using Bayesian Information Criterion to predict if DEGs are expressed in clusters and hence may establish expression networks. After predicting clusters, network analysis was performed based on mutual information of expression changes of genes using R package 'minet' [394]. Key genes within each network were predicted from centrality scores of DEGs within the networks using approaches of 'key player analysis', a method used in analyzing social networks 
[394]. Functional prediction of genes corresponding to those expression modules were assessed by mapping the genes to KEGG (Kyoto Encyclopedia of Genes and Genomes) pathways and annotating gene ontology using DAVID (https://david.ncifcrf.gov). Significant impact of DEGs on signaling pathway were determined using a pathway perturbation algorithm called 'SPIA' [329]. This topology based pathway analysis was conducted in R using ToPAseq package [395]. Functional annotation analysis was also conducted for DEGs using ToppGene Suite for gene list enrichment analysis [396].

Ligand-Receptor Analysis. Determination of ligands and their receptors was conducted using curated ligand-receptor pairs in the FANTOM5 database for human protein-coding genes [333]. Tanglegram plots of ligand-receptor expression in endometrium and conceptus were generated by 'dendextend' [397] based on hierarchical clustering of ligand and corresponding receptor expression levels separately for endometrium and conceptus. The cluster branch and nodes were color coded and black lines were used to show the correspondence between the same pair of ligand and receptor in endometrium and conceptus. The hierarchical clustering performed based on distance measured by Ward's method from expression data.

\section{RESULTS}

Experiment Overview. This experiment utilized heifers that were previously fertility classified as high fertile (HF; $100 \%$ pregnancy rate), subfertile (SF; 25-33\% pregnancy rate), or infertile (IF; $0 \%$ pregnancy rate) using serial transfer ( $\mathrm{n}=3-4$ rounds) of a single in vitro-produced embryo (Grade 1) on day 7 followed by pregnancy determination on day 28 [328]. In the present study, those same fertility-classified heifers $(H F, n=21 ; S F, n=14$; 
IF, $n=6$ ) were synchronized to estrus (day 0 ) and received two in vivo-produced embryos on day 7. All of the embryos were generated from superovulated donor cows using a single sire and were cryopreserved for direct thaw transfer. Each heifer received two grade 1 or 2 embryos from the same donor and developmental stages (a compact morula and a blastocyst). On day 17 (10 days post-embryo transfer), the entire female reproductive tract was obtained, and the uterine lumen flushed to recover the conceptus. If a conceptus was not observed in the uterine flush, the heifer was considered nonpregnant. As illustrated in Fig. 1A, the proportion of heifers from which one or two conceptuses were recovered was greater for HF $(\mathrm{P}=0.02)$ and $\mathrm{SF}(\mathrm{P}=0.03)$ heifers compared to IF heifers, but was not different $(\mathrm{P}=0.91)$ between HF and SF heifers. Accordingly, pregnancy rate was higher $(\mathrm{P}<0.05)$ in HF $(71 \%)$ and SF $(90 \%)$ than IF $(20 \%)$ heifers, but not different $(\mathrm{P}>0.05)$ between HF and SF heifers (Fig. 1B). The morphology of the conceptuses recovered from HF and SF heifers was similar and ranged from tubular to elongated and filamentous (Fig. 1C). In contrast, a single small spherical hatched blastocyst $(<0.1 \mathrm{~cm})$ was recovered from the uterus of only one IF heifer. Overall, the conceptus was longer $(\mathrm{P}<0.01)$ in HF (mean $=10.6 \mathrm{~cm}$; range $=1.2-32.2 \mathrm{~cm})$ than $\mathrm{SF}($ mean $=4.7 \mathrm{~cm}$; range $=1.5-13.5 \mathrm{~cm})$ heifers (Fig. 1D).

There were no differences in circulating progesterone concentrations on day 17 between pregnant and nonpregnant heifers $(\mathrm{P}=0.55)$ or among pregnant $\mathrm{HF}$, SF or IF heifers $(\mathrm{P}=$ 0.23) (SI Appendix, Fig. S1A \& S1B). Similarly, there was no correlation of conceptus size and circulating progesterone concentrations $(\mathrm{R}=-0.08 ; \mathrm{P}=0.80)$ (SI Appendix, Fig. S1C). 


\section{Dysregulation of Endometrial Transcriptome in Response to Pregnancy.}

Transcriptome analysis was performed using total RNA isolated from the day 17 endometria of nonpregnant and pregnant fertility-classified heifers. Sequencing of the 25 RNA-seq libraries ( $n=5$ per group) generated 27.2 to 42.8 million quality reads that mapped at a $\sim 97 \%$ rate to the Bos taurus reference genome (assembly UMD3.1). EdgeR robust analysis was used to identify differentially expressed genes (DEGs) (FDR P $<0.05)$ in the endometria, and a gene was defined as expressed if it presented $>1$ fragments per kilobase of transcript per million mapped reads (FPKM). Similar to our previous study of endometrial biopsies from these fertility-classified animals on day 14 [328], there were relatively few genes that were different $($ FDR $\mathrm{P}<0.05)$ in the endometrium of the nonpregnant HF, SF and IF animals (Fig. 2, Fig. 3A) (SI Appendix, Datasets S1, S2, S3). Functional analysis of those DEGs did not identify any significantly enriched gene ontology (GO) terms or biological pathways. Several of the DEGs in HF and SF as compared to IF endometrium encode proteins involved in immune responses or immunoglobulins (Fig. 3A). In SF compared to IF endometria, some of the 43 DEGs encode secreted proteins (GRP, IGFBP2, LYZ1, SERPINB7), intracellular enzymes (PLA2G2F), and transporters for water (AQP5) or amino acids (SLC2A3).

Comparison of the endometrial transcriptome of pregnant HF to pregnant SF animals detected 168 DEGs (Fig. 2) (SI Appendix, Dataset S4). Of note, none of the DEGs were classical ISGs (i.e. IFIT1, IFIT2, ISG15, MX2, RSAD2, OAS2) that are induced or considerably up-regulated by IFNT from the elongating conceptus [320] (Fig. 3B). The 168 DEGs were enriched for GO molecular function (extracellular matrix or ECM structural constituent), biological process (ECM organization, cell adhesion, collagen 
catabolic process, locomotion, cellular response to endogenous stimulus), and cellular component (ECM, extracellular space, membrane region) terms (SI Appendix, Table S1). Pathway analysis found enrichment in ECM organization and collagen formation. Genes increased in pregnant $\mathrm{HF}$ as compared to SF endometrium included a water transporter (AQP8), lipid transporter (FABP3), and secreted protease (MEP1B). Genes decreased in $\mathrm{HF}$ as compared to SF endometrium include cell signaling (CAMK1G, IRS4), ECM constituents, and cell adhesion molecules (SI Appendix, Dataset S4).

Endometrial responses to pregnancy were assessed by comparing the transcriptome of nonpregnant to pregnant animals (Fig. 2, Fig. 3B) (SI Appendix, Datasets S5 and S6). This analysis found 3,422 DEGs (1,680 increased, 1,742 decreased) in HF heifers and 1,095 DEGs (833 increased, 262 decreased) in SF animals with 848 genes commonly responsive to pregnancy in $\mathrm{HF}$ and SF animals (Fig. 3B). Thus, $\sim 20 \%$ of total expressed genes respond to pregnancy in the endometrium of HF animals, but only $\sim 6 \%$ of genes in endometrium of SF animals. Of note, the number of up- and down-regulated genes specific to endometrium of HF animals was 6- and 20-fold greater, respectively, than in SF animals. The lower number of DEGs in SF animals was not due to higher levels of variation in the endometrial transcriptome, as gene expression was most variable in the endometrium of pregnant HF as compared to SF animals (SI Appendix, Fig. S2). An impact analysis of the DEGs was performed based on pathways topology [329]. This analysis found that specific signaling pathways were either negatively or positively impacted in response to pregnancy in HF and SF heifers (SI Appendix, Table S2). Of note, the ECM-receptor interaction pathway was negatively impacted by pregnancy only in HF animals. These findings 
support the idea that the endometrial response to pregnancy, e.g. the conceptus, is considerably altered in SF animals.

Pathways Impacted by Pregnancy. Many of the 664 commonly responsive to pregnancy genes in HF and SF animals were classical type I ISGs stimulated by IFNT (MX2, ISG15, RSAD2, BST2, IFIT1, IFIT2, IDO1, ISG20, OAS2, IFIT3, etc.) or established progesterone- and conceptus-responsive genes (e.g. DKK1, FABP3, MEP1B, LGALS3BP) [108] (Fig. 3B) (SI Appendix, Datasets S5 and S6). Functional analyses of those 664 up-regulated genes found enrichment for many GO terms including molecular function (chemokine receptor binding, chemokine activity, peptidase activity), biological process (innate immune response, defense response, response to cytokine, response to type I IFN, response to virus), and cellular component (other organism, extracellular space, proteasome core complex) (SI Appendix, Table S3). As expected, pathways associated with the 664 commonly up-regulated genes included IFN signaling, IFNA/B signaling, cytokine signaling in the immune system, IFNG signaling, ISG15 antiviral mechanism, complement pathway, and toll-like receptor signaling (SI Appendix, Tables S1 and S3), as they are linked to IFNT actions within the endometrium. Analysis of the 184 commonly down-regulated genes found they were enriched for many GO terms including molecular function (ECM structural constituent, integrin binding, cell adhesion binding, glycosaminoglycan binding), biological process (ECM organization, collage catabolic process, cell adhesion), and cellular component (proteinaceous ECM, ECM, collagen, basement membrane, extracellular space) (SI Appendix, Tables S1 and S4). Pathways associated with the 184 commonly down-regulated genes included ECM organization, collagen biosynthesis and modifying enzymes, collagen formation, ECM-receptor 
interaction, ECM degradation, integrin signaling pathway and focal adhesion (SI Appendix, Tables S2 and S4).

The representation of enriched GO terms and pathways was substantially different between HF and SF heifers with respect to genes responsive to pregnancy (Fig. 3B) (SI Appendix, Datasets S5 and S6, Tables S1, S2, S5, S6 and S7). The 1,016 uniquely upregulated genes in the endometrium of pregnant $\mathrm{HF}$ as compared to nonpregnant $\mathrm{HF}$ animals were enriched for GO terms including molecular function (ubiquitin-like protein ligase binding, enzyme binding, RNA binding), biological process (carboxylic acid metabolic process, cellular catabolic process, innate immune response), and cellular component (mitochondrion, proteasome core complex) (SI Appendix, Tables S1 and S5). The 1,558 uniquely down-regulated genes in the endometrium of HF pregnant as compared to nonpregnant $\mathrm{HF}$ animals were enriched for $\mathrm{GO}$ terms including molecular function (protein complex binding, ECM structural constituent, cell adhesion molecule binding), biological process (ECM organization, vasculature development, biological adhesion, cell adhesion), and cellular component (proteinaceous ECM, ECM, cell junction, cell-cell junction, focal adhesion) (SI Appendix, Tables S1 and S6). Pathways associated with the 1,558 down-regulated genes in pregnant HF endometrium included ECM, ECM organization, collagen formation, structural components of basement membranes, focal adhesion, ECM-receptor interaction, and integrin signaling pathway (SI Appendix, Tables S2 and S6).

As provided in SI Appendix, Tables S1 and S7, GO analysis of the 169 genes uniquely up-regulated in the endometrium of SF heifers in response to pregnancy revealed enrichment in GO terms including molecular functions (amide transmembrane transporter activity, peptidase regulator activity) and cellular component (cell surface, extracellular 
space, plasma membrane). No biological pathways were enriched in those genes. The 78 genes uniquely down-regulated in the endometrium of SF heifers revealed no enrichment in GO terms or pathways. Collectively, these findings strongly support the idea that the endometrial response to pregnancy, e.g. the conceptus, is considerably altered in SF animals.

Alterations in the Transcriptome of HF and SF Conceptuses. Transcriptional profiling was conducted using individual conceptuses from HF ( $\mathrm{n}=17)$ and SF heifers ( $\mathrm{n}=10)$ (Fig. 2). RNA sequencing generated 36.5 to 65.5 million quality reads with a $\sim 92 \%$ mapping rate to the Bos taurus reference genome. EdgeR robust analysis identified 1,287 DEGs (558 increased, 729 decreased) in HF as compared to SF conceptuses (SI Appendix, Fig. S4 and Dataset S7). In order to ensure that the observed differences were not related to size of the conceptuses [94, 330], conceptuses from only HF heifers were categorized as long (range $=9.8-32.2 \mathrm{~cm} ; \mathrm{n}=10)$ or short $($ range $=1.2-6.9 \mathrm{~cm}, \mathrm{n}=7)$. Subsequent EdgeR robust analysis found only 18 DEG (7 increased, 11 decreased) between the long and short conceptuses from HF heifers (SI Appendix, Dataset S8). Thus, the differences in the transcriptome between HF and SF conceptuses is not due to their length [94, 330], but rather the fertility phenotype of the uterus.

Functional annotation of genes between HF and SF conceptuses identified many biological processes enriched in DEGs including growth and regulation of actin cytoskeleton in the DEGs increased in HF conceptuses (SI Appendix, Tables S8 and S9). GO analysis of the 558 genes increased in HF conceptuses found enrichment for molecular function (signal transducer activity, transcription factor activity, transcriptional activator activity) and biological process (embryo development, embryonic morphogenesis, animal 
organ morphogenesis, epithelium development, receptor signaling, tissue morphogenesis, morphogenesis of an epithelium), but no cellular component terms (SI Appendix, Tables S8 and S9). The 558 genes increased in HF conceptuses were enriched for pathways including integrin-mediated cell adhesion, signaling by FGFR, signaling by NGF, signaling by FGFR, focal adhesion, FGF signaling, estrogen signaling, ErbB1 signaling, insulin signaling, and MAPK signaling. GO analysis of the 729 genes increased in SF conceptuses found enrichment for molecular function (molecular function regulator, receptor binding, sialyltransferase activity, heparin binding), biological process (organ development, cellular lipid metabolic process, cell adhesion, lipid metabolic process), and cellular component (extracellular space, ECM, apical part of cell, proteinaceous ECM) terms (SI Appendix, Tables S8 and S10). Pathways for the 729 up-regulated genes in SF conceptuses were enriched for ECM and ECM-associated proteins.

In order to illuminate possible mechanisms impacting loss of conceptuses in the SF animals, the Mouse Genomic Informatics Knockout database [331] was queried to determine if genes down-regulated in SF as compared to HF conceptuses are associated with embryonic lethality. As summarized in SI Appendix, Table S11, many of the genes decreased in SF conceptuses have knockout database annotation terms corresponding to "embryonic lethality during organogenesis, complete penetrance", "embryonic lethality during organogenesis", "abnormal vascular development", "abnormal embryonic tissue morphology", "abnormal prenatal growth/weight/body size“, "abnormal vitelline vasculature morphology", and "lethality throughout fetal growth and development, complete penetrance". 
Network analyses of DEG in HF and SF Conceptuses. Mutual information based network analyses were conducted by implementing a model based cluster approach. Using Bayesian Inference Criterion as model selection [332], we identified 9 gene expression clusters in conceptus transcriptome and predicted gene networks of those clusters from DEGs discovered in conceptus of HF as compared to SF animals. Using network centrality method, we predicted top key players within each cluster that may play central role in gene expression networks in HF and SF conceptuses (SI Appendix, Table S12). This analysis revealed many key players including critical enzymes (FADS1, PTGS2), transcription factors (EFHD2, IRX4, ZBBTB7B) as well as several secreted proteins (TKDP1, TKDP4, SSLP1).

Conceptus-Endometrial Signaling. Conceptus-endometrial interactions during elongation primarily involve secreted factors from the trophectoderm and endometrial epithelium [117, 315]. The GO extracellular space term (GO:0005615) was used to determine gene products secreted from the endometrium or conceptus into the uterine lumen based on DEGs in the endometrium (pregnant vs nonpregnant) and conceptus from HF and SF animals (SI Appendix, Table S13). This analysis revealed substantial differences in genes encoding secreted proteins. For instance, expression of 206 genes were increased and 296 were decreased in the endometrium by pregnancy in HF animals, but only 36 genes were increased and 5 decreased by pregnancy in SF animals. Further, 79 genes were increased in HF conceptuses and 173 were increased in SF conceptuses.

A network of ligand-receptor-mediated multicellular signaling from the FANTOM5 database of human cells [333] was used to determine ligands and receptors expressed by the endometrium and conceptus from HF and SF animals (SI Appendix, 
Datasets S9 and S10) and is represented in Fig. 4. Tanglegram plots of ligand-receptor expression (SI Appendix, Fig. S3) found that SF heifers have relatively higher variation in branch lengths ( 0 through 300,000) of receptor-ligand clusters for conceptus than those of HF animals (0 through 250,000). Thus, ligand-receptor expression in SF conceptuses is more discordant in their correspondence with endometrium ligand-receptors compared to HF animals, which was measured from distance measure (Ward's method) of gene expression variation of receptors compared to ligands between endometrium and conceptus in SF and HF groups separately. As illustrated in Fig. 4 and SI Appendix, Fig. S3, some receptors are expressed with higher expression of ligands in both in conceptus and endometrium, but in the majority of cases there is a negative regulation of receptor-ligands both within and between endometrium and conceptus (SI Appendix, Table S14). However, there was a relative lack of receptor-ligand interactions between conceptus and endometrium in SF as compared to HF animals. Further, ligands and receptors were not as reciprocal in the SF as compared to HF animals and more ligands in the endometrium were uniquely increased or decreased by pregnancy in HF as compared to SF animals. The same result was found for receptors in the endometrium. Differential expression of ligands and receptors was also observed in the conceptus from HF and SF animals. The alterations in ligands and receptors in the endometrium and conceptus would impact paracrine and autocrine signaling for conceptus growth and endometrial receptivity.

Based on protein age estimates, protein ligands are generally evolutionary younger than the cognate receptors [333]. The age of proteins of Bos taurus were assessed from ProteinHistorian analysis [334] that predicts age from evolutionary conservation of proteins. Next, a contingency test of up-regulated versus down-regulated genes in day 17 $\mathrm{HF}$ and SF endometrium was conducted by differentiating them based on 'age' of the 
encoded protein (SI Appendix, Fig. S5). This analysis revealed that the transcriptome response of HF and SF pregnant heifer endometrium is associated with protein age. In HF pregnant heifers, the endometrium transcriptome did not display age differences in encoded proteins, but age of proteins was different (Pearson Chi Square $=3.37, \mathrm{P}=0.04)$ in $\mathrm{SF}$ animals. The genes up-regulated in SF endometrium are biased $(\mathrm{P}=0.0064)$ towards younger age. Collectively, these analyses of ligands and receptors further support the idea that the endometrium and conceptus is asynchronous in SF animals.

\section{DISCUSSION}

Our previous study of the fertility-classified heifers used here found no difference in pregnancy rate or conceptus development on day 14 (7 days post-embryo transfer) [328]. It has long been postulated that pregnancy loss in heifers and non-lactating cows was primarily due to defects in conceptus survival and elongation between conception or embryo transfer (day 7) and day $16[36,335]$. In the present study, conceptus survival was substantially compromised in IF heifers on day 17 (10 days post-embryo transfer), but not in SF as compared to HF animals. Given that SF animals have substantially increased embryo mortality by day 28 , these findings suggest that embryonic loss in heifers after embryo transfer occurs primarily between days 17 and 28 during the implantation period of pregnancy. True infertility occurs in only $5 \%$ or so of heifers, and SF is more pervasive and costly in beef and dairy animals [336-338]. Although pregnancy rates and embryo survival were not different on day 17 in SF and HF animals receiving two embryos on day 7, the conceptus was two-fold longer in HF than SF animals on day 17 in the present study. The variability in recovered conceptus size and morphology was striking (Fig. 1), but is consistent with many other studies of beef and dairy heifers and cows $[36,94,95,330,339$, 
340]. In the present study, embryonic loss in the SF heifers had to occur between days 17 and 28, which encompasses the implantation period of pregnancy [341-343]. Recent data from dairy heifers and cows as well as somatic cell nuclear transfer pregnancies supports the hypothesis that embryonic loss is most prevalent during this period [2, 57, 344, 345]. However, little is known of the biological pathways and gene networks regulating implantation and placentation in cattle $[342,345,346]$. Progesterone action via endometrium is critical for conceptus development and elongation [315, 320] and increased post-ovulatory progesterone concentrations is associated with increased conceptus growth on days 13 and 14 [347, 348]. In agreement with our recent findings [328, 349], differences in levels of circulating progesterone concentrations were not associated with pregnancy outcomes. This reinforces our hypothesis that mechanisms other than circulating progesterone concentrations are associated with pregnancy loss in the fertility-classified SF and IF heifers.

A prevailing theory is that smaller conceptuses producing less IFNT are inferior in their ability to establish pregnancy as compared to longer conceptuses that produce more IFNT [350]. That theory has been difficult to test given the difficulties in retrieving elongating conceptuses and transferring them into a recipient uterus without damage [95]. Despite differences in the size of HF and SF conceptuses, the response of the endometrium to the conceptus was not different with respect to induction of classical ISGs by IFNT, such as ISG15, MX2, etc., which is not unexpected given that very little IFNT is needed to maximally stimulate expression of ISGs in cells and tissues [351]. In fact, the absolute amount of IFNT that must be produced by the conceptus to signal pregnancy recognition has never been established [108]. As the pregnancy recognition signal, IFNT acts in a paracrine manner on the endometrial lumenal epithelium to regulate expression of the 
oxytocin receptor (OXTR) gene, as OXTR are key for endometrial generation of luteolytic pulses of prostaglandin F2 alpha $[108,113]$. In cattle, OXTR expression increases in the endometrial lumenal epithelium after day 14 in nonpregnant but not pregnant cattle [352]. In the present study, endometrial OXTR mRNA levels were not different among nonpregnant HF, SF and IF heifers and, as expected, were lower in the endometrium of pregnant (mean 2.5 FPKM) as compared to nonpregnant (mean 19.5 FPKM) HF heifers. In SF heifers, OXTR mRNA levels were not different in the endometrium of pregnant (mean 3.8 FPKM) and nonpregnant (mean 2.1 FPKM) animals. Although OXTR expression was clearly different in SF heifers on day 17 post-estrus, no consistent difference in estrous cycle length was observed in our previous study of these SF and IF heifers [328].

Transcriptional profiling of the endometrium was conducted to begin understanding the biology of pregnancy loss in IF and SF animals. Minimal differences were observed in the nonpregnant endometrial transcriptome amongst IF, SF and HF heifers. Similarly, minimal gene expression differences were found in endometrial biopsies from these same fertility-classified heifers on day 14 (7 days post-embryo transfer) [328]. Few differences in the endometrium were also detected in endometria from fertilityclassified heifers on day 13 post-estrus [349] or day 14 post-estrus [353] and also endometrial biopsies from lactating dairy cows on day 13 post-estrus [146]. The lack of conserved differences among these studies could be attributed to a myriad of factors including how fertility was classified, breed effects, and endometrial sampling technique. Nonetheless, RNA-seq analysis detect some genes increased in HF or SF as compared to IF endometrium that encode factors involved in reproductive tract defense against pathogens and immune system modulation (CTLA4, IFI47, IGJ, IGM, MUC13). In other 
mammals, the embryo induces expression of molecules in the endometrium that function to suppress the immune response and/or promote tolerance to the embryo [345]; however, relatively little is known about the immunology of pregnancy in cattle [354]. In summary, analyses of the nonpregnant endometrium transcriptome did not provide significant insights into the biological pathways underlying embryo mortality in IF and SF animals. Similar to studies of humans with fertility problems [355], the gene expression signature in nonpregnant enodmetrium may not be useful to predict fertility phenotype and pregnancy outcome. Further studies on the genome [349] or uterine secrotome of IF animals may provide insight into failure of conceptus survival and growth in those animals $[356,357]$.

Of the 44 genes decreased in pregnant SF as compared to HF heifers, several of them encode secreted factors (FABP3, MEP1B) and transporters (SLCO4C1, SLC7A1) implicated in conceptus implantation in ruminants. MEP1B is a zinc metalloendopeptidase that is expressed by and secreted from epithelial cells. In the bovine uterus, it is induced by progesterone in the endometrial glands and implicated in elongation of the conceptus [347]. FABP3 is involved in the uptake, intracellular metabolism and transport of long-chain fatty acids and up-regulated by progesterone in the endometrial lumenal epithelium between days 13 and 16 in cattle [317]. Long chain fatty acids are important for cell growth and production of eicosanoids, which are important for conceptus elongation in ruminants [189, 358]. SLCO4C1 is an organic ion transporter that is involved in the membrane transport of eicosanoids. Analysis of the conceptus transcriptome data identified PTGS2 as a key player in the present study, and PTGS2 is critical for conceptus elongation and pregnancy establishment in sheep [359] and a predictor of embryo viability and pregnancy success in cattle [360]. SLC7A1 is an arginine transporter up-regulated in the lumenal epithelium of 
the ovine uterus during early pregnancy, and arginine stimulates trophectoderm cell proliferation, migration and IFNT production in vitro [361]. Functional analysis of the 44 genes found enrichment for biological processes involved in the inflammatory response (MEP1B, S100A12, C2, IL17RC, CFI, IDO1, NUPR1, TFRC). Controlled inflammation is important for the establishment of pregnancy in other mammals, although the immunological underpinnings of pregnancy are not well understood in ruminants [354, 362].

Transcriptome analyses uncovered the remarkable finding that the endometrial response to the conceptus was extensively altered in SF animals with an approximate threefold disparity in DEGs. Comparison of the nonpregnant and pregnant endometrium revealed 2,574 unique DEGs in HF animals and 247 unique DEGs in SF animals. Further, 848 DEGs commonly responded to pregnancy including classical ISGs up-regulated by IFNT (CLEC4F, MX2, ISG15, RSAD2, IFIT1, OAS2, etc.) as well as a number of known progesterone-induced and conceptus-stimulated genes (FABP3, MEP1B, LGALS3BP, SOCS3, S100A2). Thus, the endometrium of SF animals appears to respond appropriately to progesterone and IFNT, which are the major established regulators of endometrial gene expression and function to date in ruminants $[108,320]$. Comparisons of the endometrium from pregnant HF and pregnant SF heifers as well as the differential response of the endometrium of HF and SF heifers to pregnancy revealed substantial alterations in genes regulating ECM structure and organization as well as cell adhesion, cell-matrix adhesion, and cell movement. Collectively, results support the idea that ECM remodeling is a normal response of the endometrium to the elongating and implanting conceptus, but this remodeling is substantially diminished in SF animals. Indeed, genes enriched in GO terms for cell adhesion, ECM, basement membrane and vasculature development were down- 
regulated in pregnant as compared to cyclic cows [363, 364]. Differences in ECM remodeling were found in comparisons of the transcriptomes of chorionic, endometrial or placental tissues from natural as compared to embryo transfer or somatic cell nuclear transfer-derived pregnancies that are predominantly loss during implantation and placentation during the first few months [345]. Implantation and placentation involve remodeling of the endometrial and chorionic ECM [342, 365, 366]. Endometrial and fetal tissues undergo major tissue remodeling during this period to aid the proliferation, differentiation, and migration of binucleate cells, which are regulated by locally produced MMPs and tissue inhibitor of matrix metalloproteinase 1 [367-370]. The organization of the ECM is a complex process which involves interactions between the ECM components and extracellular proteoglycans that undoubtedly impact growth and development of the placentomes. The mechanisms of pregnancy loss in SF heifers appears to be associated with the process of ECM remodeling and impaired conceptus endometrium interactions. ECM and adhesion proteins increased in pregnant SF endometrium are normally decreased in HF endometrium by pregnancy. Trophectoderm attachment and adhesion to the endometrial lumenal epithelium is a fundamental event in implantation and pregnancy establishment in mammals [371] and thus likely disrupted in SF animals. Excessive ECM in SF animals could possibly inhibit the embryonic adhesion to endometrial tissue [372].

Many of the 729 genes down-regulated in SF conceptuses are enriched for GO biological processes that include embryo development, embryo morphogenesis, animal organ morphogenesis, epithelium development, and morphogenesis of an epithelium among others and associated with embryonic lethality or abnormal implantation in mice. Several genes down-regulated in the SF conceptus (GCM1, BMP2, FGFR2) are postimplantation embryonic lethal in null mice due to defects in placental development (SI 
Appendix, Table S11). Of note, GCM1 is a critical transcription factor that regulates placental development and trophoblast differentiation in mice and humans [373]. Further, genes down-regulated in the SF conceptuses were enriched for GO molecular functions involving transcription factors activity and transcriptional activator activity; however, key transcription factors regulating conceptus elongation and placental development are not known in cattle [342]. A number of biological processes were also down-regulated in the SF conceptus included integrin-mediated cell adhesion, focal adhesion, FGF and FGF signaling, and MAPK signaling, which are all important pathways for conceptus growth and implantation [374]. Alterations in WNTs and DKK1, a WNT inhibitor, was observed in the SF conceptus, and canonical and noncanonical WNT signaling pathways are conserved regulators of conceptus-endometrial interactions in mammals and implicated in conceptus elongation and implantation in sheep and cattle [375, 376]. FGFR2 was decreased in the SF conceptus, and FGF2 from the endometrium activates FGFR2 in the elongating bovine conceptus and increases trophectoderm proliferation and IFNT production [135]. Factors involved in cell migration and elongation (GJA5, TSPAN1, GJB5, ITGB2, PECAM1) were also decreased in the SF conceptus. Thus, the developmental program of conceptus growth and differentiation is likely compromised in SF animals and leads to post-elongation embryonic mortality due to defects in extraembryonic tissues (placenta, allantois) and/or the embryo itself.

In addition, 558 genes up-regulated in SF conceptuses are enriched for GO molecular function (molecular function regulator, receptor binding, heparin binding) and cellular component (extracellular space, extracellular matrix). Altered secretion of proteins that are ligands for receptors expressed on the conceptus and/or endometrium could lead to defects in conceptus development and implantation [345]. This supposition is supported 
by in silico determination of secreted factors and ligands and their differences in endometrium and conceptus of pregnant HF and SF animals. Indeed, the endometrium is an early biosensor and distinct endometrial responses are elicited by embryos produced by artificial means [345, 377, 378]. Results of the present study suggest that other factors besides IFNT are important mediators of conceptus-endometrial interactions during early pregnancy. Bioinformatics analysis identified several secreted proteins (TKDP1, TKDP4, SSLP1) as key players in conceptus gene expression networks. The trophoblast kunitz domain proteins (TKDP) are a multigene family that are predominantly expressed in the trophoblast cells of the bovine placenta during early pregnancy [379]. Other potential important signaling molecules from the conceptus include BMP2, BMP5, GDF6, and GDF11, which activate signaling pathways important for endometrial function and implantation in other mammals $[380,381]$.

Collectively, the transcriptional profiling results strongly support the idea that conceptus-endometrial interactions are dysregulated in SF animals and underlies the observed pregnancy loss by day 28 . This hypothesis is strongly supported by studies in cattle, mice and humans finding that dysregulated interactions between the conceptus and uterus have adverse ripple effects resulting in pregnancy loss, miscarriage, or preeclampsia $[382,383]$. Implantation requires carefully orchestrated interactions between the receptive endometrium and elongating conceptus, and studies of somatic cell nuclear transferderived pregnancies in cattle clearly support the idea that altered conceptus-endometrial interactions is a cause of post-elongation pregnancy loss [345, 378, 384]. Given that only minimal differences were observed on conceptus transcriptome when short and long HF conceptuses were compared, it is likely that the differences in the conceptus transcriptome of HF and SF conceptuses arise initially from influences of the endometrium. 
Hypothesis for the Loss of Pregnancy in Infertile and Subfertile Animals. Pregnancy loss during the first trimester of gestation of pregnancies established by embryo transfer has been hypothesized to involve failures or delays in conceptus elongation and/or embryonic development resulting in loss of pregnancy by day 30 [2]. The present studies were conducted with beef heifers that were fertility-classified by embryo transfer and whose pregnancy rates were predicated on innate uterine competence to support pregnancy establishment. The defects in conceptus survival and elongation observed on day 17 in the IF heifers support the idea that an incompetent uterus is present in $5 \%$ or so of cattle, but they did not illuminate why the IF uterus fails to support pregnancy. Subfertility is a more prevalent issue in cattle, and studies here found that conceptus survival was not compromised in SF heifers, indicating that pregnancy loss occurs between days 17 and 28 in SF animals during the implantation period. Findings from the transcriptome analyses clearly support the idea that conceptus-endometrial interactions are dysregulated in the SF animals. Thus, our studies strongly support the adverse ripple effect hypothesis that aberrant communication between the endometrium and conceptus disrupts normal implantation and placentation processes leading to pregnancy loss and later pregnancy complications [382]. Based on the experimental approach of fertility classification here, we propose that the alterations in the SF conceptus transcriptome result from inappropriate responsiveness of the endometrium to the conceptus in SF animals. The alterations in endometrial and conceptus gene expression are likely caused, in part, by alterations in DNA methylation [385-389]. Subsequent pregnancy loss occurs due to the ripple effects of dysregulated conceptus-endometrial interactions due to insufficient: (a) conceptus attachment and adhesion to the endometrium; (b) placental development as a consequence 
of inadequate endometrial remodeling or defects in allantois growth and development; and/or (c) embryogenesis. In summary, the studies here provide an important foundation to understand implantation and early placentation phase pregnancy loss and develop genetic and physiological approaches to improve the outcome of natural and assisted pregnancies.

\section{ACKNOWLEDGMENTS}

This work was supported by 1 R01 HD072898 from the Eunice Kennedy Shriver National Institute of Child Health and Human Development. 


\section{FIGURES}
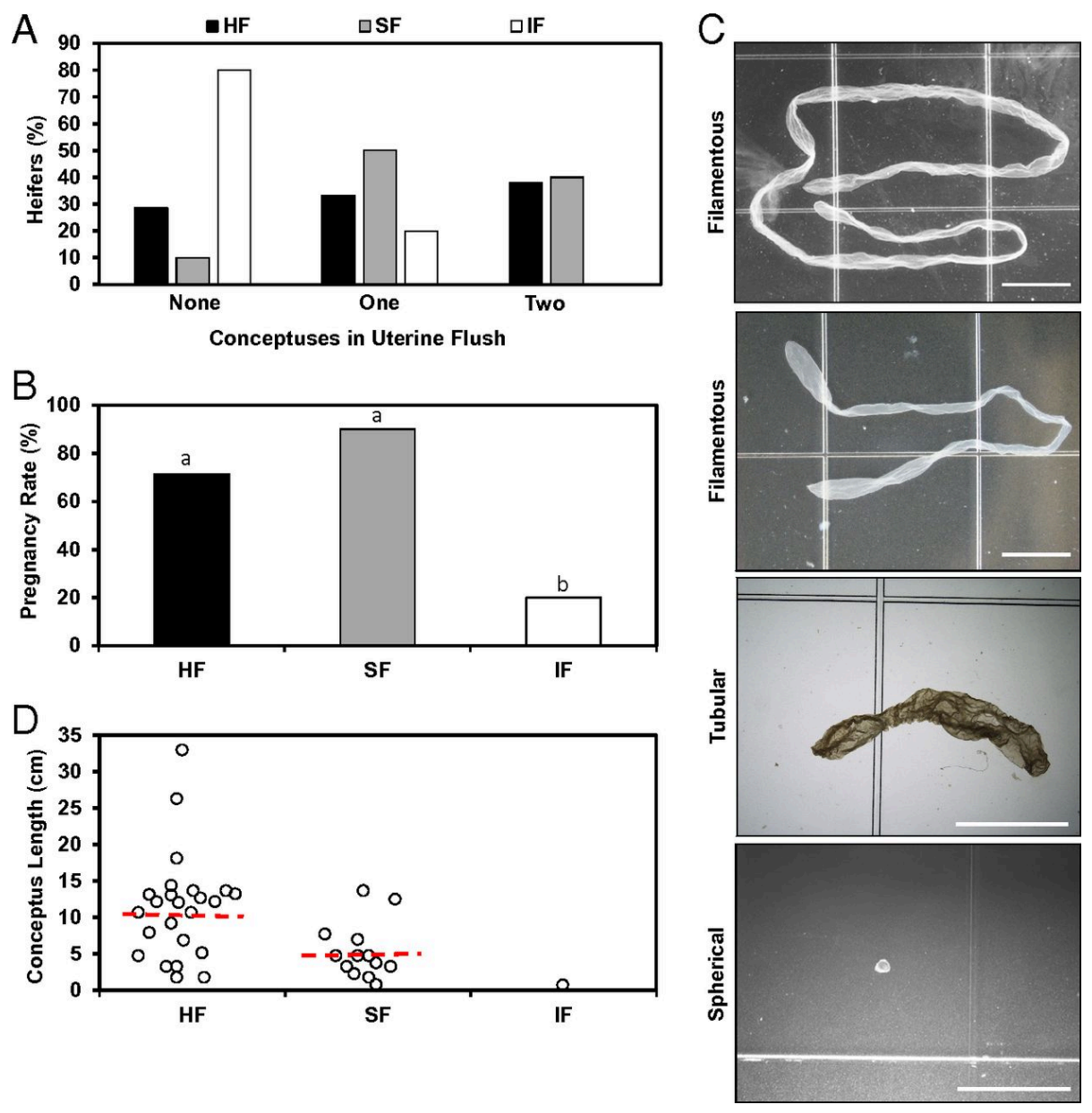

\section{Figure 1. Day 17 pregnancy outcome in fertility-classified heifers.}

High fertile (HF), subfertile (SF) and infertile (IF) heifers were synchronized and received two in vivo produced embryos on day 7 post-estrus. The reproductive tract was acquired on day 17 (10 days post-embryo transfer) and flushed to recover the conceptus(es). If present, conceptus morphology and length were determined using a stereomicroscope. A) Percentage of heifers with an identifiable conceptus in the uterine flush. B) Column graph showing pregnancy rate (y-axis) of the fertility classified heifers (x-axis). C) Representative conceptus morphology recovered from heifers. Note a single spherical embryo of less than $1.5 \mathrm{~mm}$ was recovered in one IF heifer. Bar $=1 \mathrm{~cm}$. D) Conceptus 
length was longer $(\mathrm{P}<0.05)$ from $\mathrm{HF}$ as compared to $\mathrm{SF}$ heifers. Individual conceptus length is plotted with the mean length denoted by the dashed red line. 


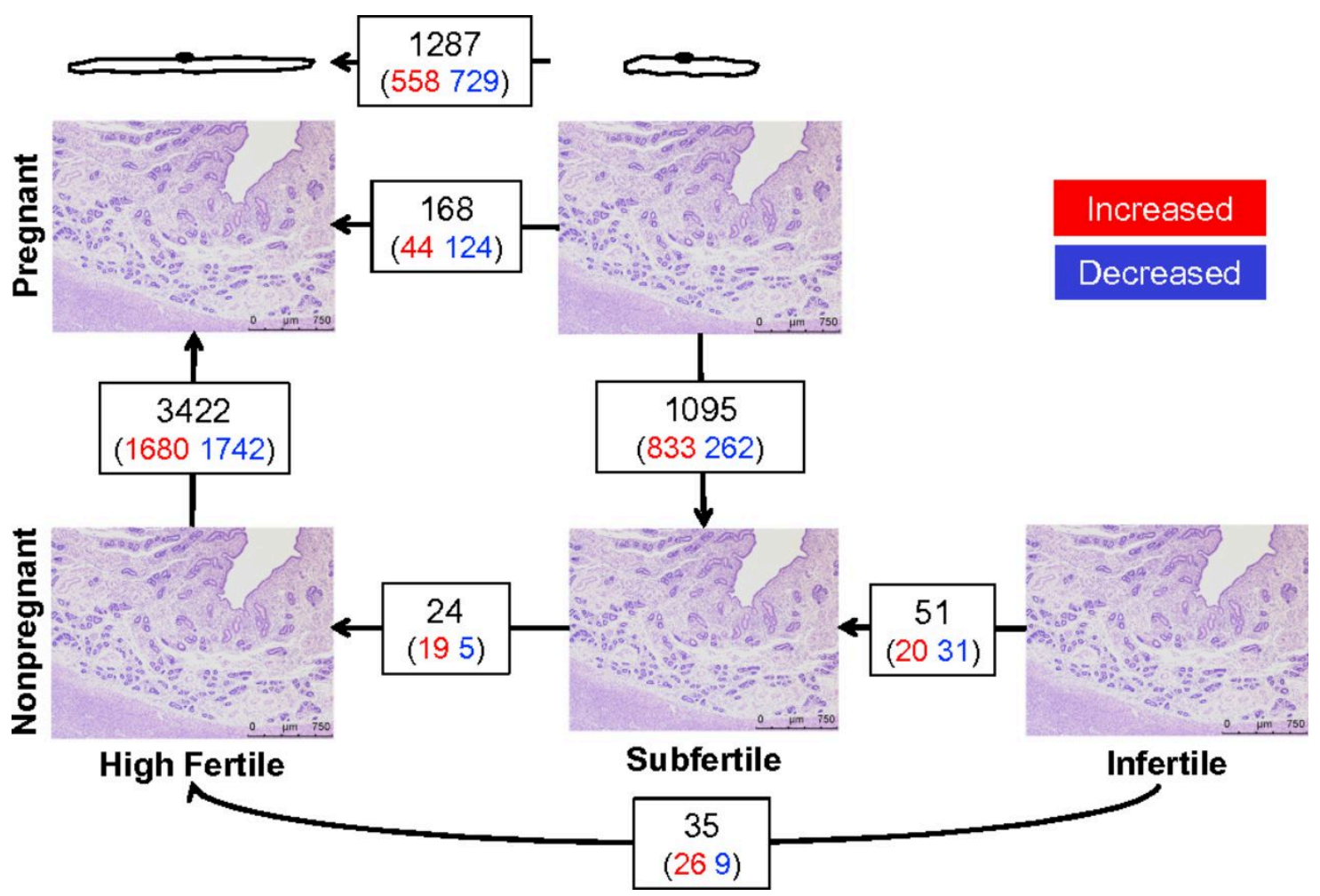

Figure 2. Summary of endometrium and conceptus transcriptome analyses.

Differentially expressed genes (FDR $\mathrm{P}<0.05)$ were determined by EdgeR robust analysis, and the number of increased and decreased transcripts are indicated in red and blue, respectively, for each comparison. 
A Nonpregnant Endometrium

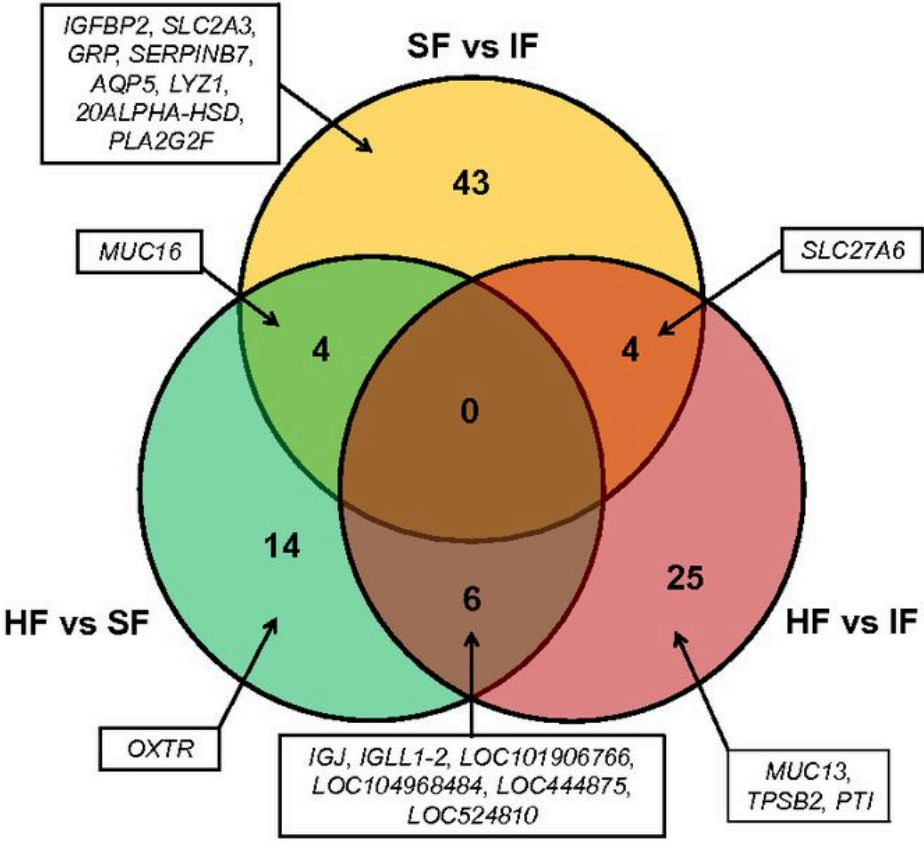

B Pregnant vs Nonpregnant Endometrium

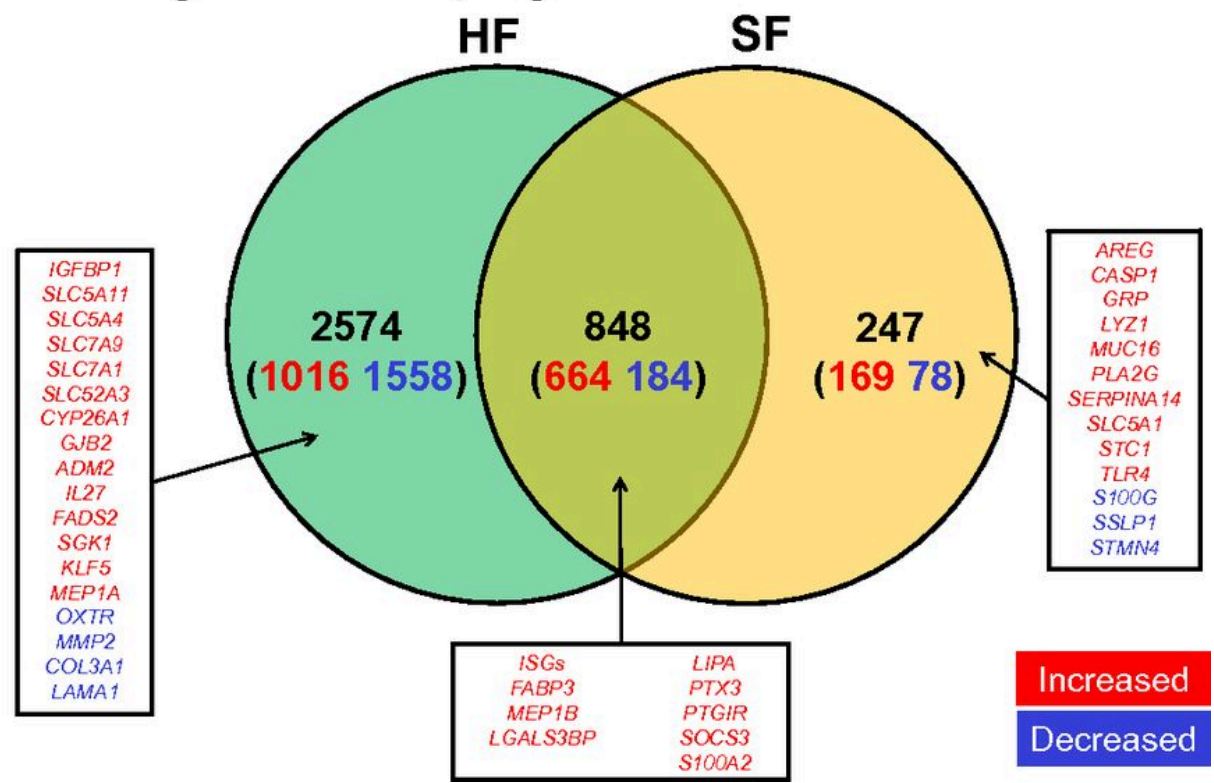

Figure 3. Transcriptome analysis of endometrium from nonpregnant and pregnant day 17 heifers.

Total RNA was extracted from five nonpregnant high fertile (HF), subfertile (SF), and infertile (IF) heifers and five pregnant HF and SF heifers. Normalized and log2 transformed 
read count data and differentially expressed genes (FDR $\mathrm{P}<0.05)$ were determined by edgeR-robust analysis. A) Venn diagram showing the number of unique or common transcripts between the endometrium of nonpregnant HF, SF and IF heifers. B) Venn diagram showing the number of unique or common pregnancy-regulated transcripts in the endometrium of HF and SF heifers. For this analysis, differentially expressed genes were determined in the endometrium of nonpregnant and pregnant HF or SF heifers and used to determine number of unique or common DEGs. 


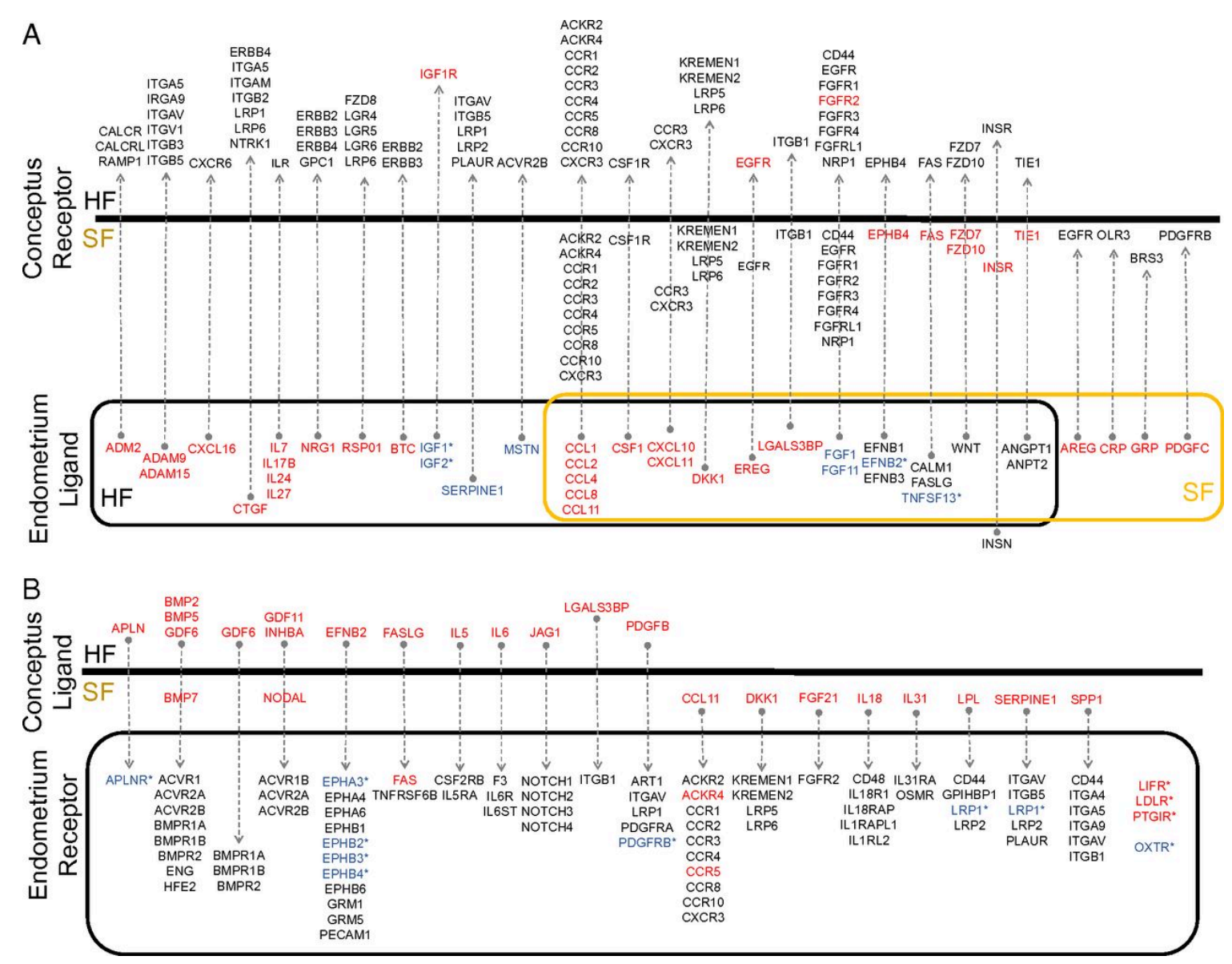

Figure 4. Receptor-ligand interactions between conceptus and endometrium at day

\section{7 of pregnancy in high fertile (HF) and subfertile (SF) heifers.}

(A) Diagram of select ligands from the endometrium and receptors in the conceptus. The endometrium ligand (on bottom) are shown as either commonly differentially expressed in both HF and SF endometrium (overlapping box) or exclusively to pregnant HF (black box) or SF (orange box) endometrium. (B) Diagram of select ligands from the conceptus and receptors in the endometrium. Genes shown in red have higher expression levels than those in black, and those in blue have lower expression than those in black. 


\section{SECTION IV:}

Analysis of the Uterine Lumen from Fertility-Classified Heifers: I.

Glucose, Prostaglandins and Lipids

Joao G.N. Moraes, Susanta K. Behura, Thomas W. Geary and Thomas E. Spencer 


\begin{abstract}
Preimplantation growth of the conceptus (embryo and associated extraembryonic membranes) is highly dependent on the substances present in the uterine lumen. Here, we investigated glucose, prostaglandins (PGs) and lipids in the uterine lumen of pregnant and nonpregnant fertility-classified heifers to explore the biological mechanisms regulating conceptus growth and survival during the time of maternal recognition of pregnancy (day 17). The transcriptional profile of endometrium and conceptuses were utilized to further explore the metabolism of glucose, PGE2, PGF2 $\alpha$ and PGI2 during early pregnancy. Differences in the transcriptome were observed between conceptuses collected from heifers classified as high fertile (HF) or subfertile (SF) for the expression of genes encoding glucose transporters and among genes involved with glycolysis and gluconeogenesis. Uterine luminal concentrations of glucose were increased by pregnancy, but did not differ among fertility-classified heifers. PGE2 and PGF2 $\alpha$ were increased in the uterine lumen by pregnancy, and HF heifers tended to have higher uterine luminal concentrations of PGE2, PGF2 $\alpha$ and 6-keto-PG1 $\alpha$ than SF heifers. Differences were observed in expression of genes regulating PG signaling, arachidonic acid metabolism, and PPAR signaling, among conceptuses and endometrium from fertility-classified heifers. Phosphatidylcholine was the main lipid class which increased in the uterine lumen of HF heifers by pregnancy. Expression of several genes involved with lipid metabolism differ between HF and SF conceptuses, and a number of fatty acids were differently abundant in the uterine lumen of pregnant HF and SF heifers. The observed differences in uterine luminal biology during early pregnancy are hypothesized to influence uterine receptivity and pregnancy establishment in cattle.
\end{abstract}




\section{INTRODUCTION}

The uterus clearly impacts conceptus (embryo/fetus and associated extraembryonic membranes) survival and programs its development, thus affecting pregnancy success [179, 398-401]. After hatching from the zona pellucida (days 9-10), the bovine blastocyst slowly grows into an ovoid or tubular form on days 12-14 and is then termed a conceptus [36]. The conceptus is only about $2 \mathrm{~mm}$ in length on day 13 , about $6 \mathrm{~mm}$ by day 14 , reaches about $60 \mathrm{~mm}(6 \mathrm{~cm})$ by day 16 , and $20 \mathrm{~cm}$ or more by day $19[36,95]$. The preimplantation growth of the conceptus is highly dependent on the substances present in the uterine lumen. Uterine glands are present in the endometrium of all mammals [105], and their secretions constitute an important component of the histotroph, which is essential for preimplantation conceptus development in sheep [76,77]. Histotroph in the uterine lumen of cattle has been characterized [81-85] and is a complex mixture of amino acids, glucose, lipids, proteins, vitamins, ions, cytokines, hormones, growth factors, among other substances. Little is known about how different levels of specific constituents in the uterine lumen regulates pregnancy success in cattle.

Glucose is an essential nutrient for preimplantation conceptus development [138, $139]$ and its concentration in the uterine lumen rises around time of conceptus elongation in sheep [139] and cattle [141]. Although the capacity of the uterus to generate free-glucose through gluconeogenesis is debatable [144-146], glucose transporters are thought to play an important role modulating intrauterine concentrations of glucose during pregnancy [138]. Mammalian cells express three families of glucose transporters, the facilitative glucose transporter family (solute carriers SLC2A/GLUT), the $\mathrm{Na}^{+} /$glucose cotransporter family (solute carriers SLC5A/SGLT) [136], and the recently discovered SWEET (SLC50) sugar transporter $[149,150]$, and the uterus expresses several members of these families 
[151], including SLC2A1, SLC5A1 and SLC5A11 whose expression increase in pregnant compared to cyclic endometrium in sheep [138].

Prostaglandins (PG) are lipid hormones derived from arachidonic acid [402], and are essential for conceptus development. In sheep, intrauterine infusion of meloxicam, a selective inhibitor of prostaglandin-endoperoxide synthase 2 (PTGS2), inhibited conceptus elongation [218]. Concentrations of PGs in the uterine lumen increase during early pregnancy in ruminants $[138,204]$, because both the conceptus and endometrium produce prostaglandins, and endometrial production of PGs is further stimulated by interferon tau (IFNT) $[120,205]$, the signal for maternal recognition of pregnancy which is secreted predominantly by the trophectoderm of the elongating bovine conceptus after day 15 [109]. The classical signaling of prostaglandins is through membrane-bound $\mathrm{G}$ protein-coupled receptors, and different types of PGs act through their specific receptor(s) and therefore regulates distinct biological processes $[212,213]$. Nonetheless, selected PGs can also activate nuclear receptors [206-208]; PGI2 can signals through the peroxisome proliferator-activated receptor (PPAR) delta (PPARD) [214], and 15-deoxy- $\Delta^{12,14-P G J 2, ~ a ~}$ metabolite of PGD2, can activate PPAR gamma (PPARG) $[215,216]$. PPARs are ligandregulated transcription factors that upon heterodimerization with retinoid $\mathrm{X}$ receptor (RXR) and ligand binding, regulate the expression of target genes and consequently cellular function [175].

Lipids are another important component present in the uterine lumen. In addition to being a precursor of eicosanoids [403], they play many critical roles in the body, such as serving as energy source and storage, providing substrates for membrane biogenesis, and acting as signal molecules [169]. The lipid content of the uterus fluctuates throughout the estrus cycle in several species [170] and the accumulation of lipid droplets in the 
endometrium luminal epithelium (LE) during the estrus cycle in sheep is attributed to progesterone from the ovarian corpus luteum (CL) [171, 173, 404], and this lipid accumulation has been hypothesized to serve as a reservoir to allow an increase in lipid availability in the uterine lumen during early pregnancy $[172,173]$. Progesterone also increases the number of extracellular vesicles (EV) in the uterine lumen during the period of conceptus elongation in sheep [405], and the lipid component of EVs is hypothesized to be important to support the growth of trophectoderm cells during conceptus elongation [201]. Fatty acids present in the uterine lumen likely participate in the crosstalk between the conceptus and endometrium during pregnancy, as saturated and unsaturated fatty acids can serve as ligands of PPAR receptors [172, 174-177], which are expressed by both, the bovine conceptus and endometrium [94, 179]. Although fatty acids are essential biological components, uterine lumen fatty acid constitution remains poorly understood in ruminants.

To identify changes in the uterine lumen constituents associated with increased or reduced uterine capacity to support pregnancy, the current experiment utilized heifers that were previously fertility-classified as high fertile (HF; $100 \%$ pregnancy rate), subfertile (SF; $25-33 \%$ pregnancy rate), or infertile (IF; $0 \%$ pregnancy rate) using serial transfer ( $n=3-4$ rounds) of a single IVP embryo (Grade 1) on day 7 followed by pregnancy determination on day 28. Interestingly, conceptus development and survival by day 14 (7 days post-transfer) was not different among fertility-classified heifers and only minimal differences in their endometrium transcriptome were observed on day 14 [328]. Nonetheless, in a subsequent study, we demonstrated that on day 17 (10 days post-transfer), pregnancy rate was higher in HF (71\%) and SF (90\%) than IF (20\%) heifers. Day 17 conceptuses were longer in HF (mean $10.6 \mathrm{~cm}$; range 1.2-32.2 cm) than SF (mean $4.7 \mathrm{~cm}$; range $1.5-13.5 \mathrm{~cm}$ ) heifers, and the endometrial and conceptus transcriptome was 
dysregulated in SF heifers [179]. Because differences in endometrial transcriptome can translate into differences in the uterine lumen constituents [192], the focus of the current study was to investigate concentrations of glucose, prostaglandins (PGE2, PGF2 $\alpha$ and 6keto-PG1 $\alpha$ metabolite of PGI2 $[406,407])$ and lipids in the uterine lumen from fertilityclassified heifers, to gather information related to the mechanisms influencing conceptus development and survival in heifers with distinct uterine capacity to support pregnancy, and to detect differences in uterine lumen constituents that are induced by pregnancy.

\section{MATERIALS AND METHODS}

Animals. All animal procedures were conducted in accordance with the Guide for the Care and Use of Agriculture Animals in Research and Teaching and approved by the Institutional Animal Care and Use Committees of the USDA-ARS Fort Keogh Livestock and Range Research Laboratory and the University of Missouri. All fertility-classified heifers participating in the study were housed in the same pasture at the Beef Research and Teaching Farm of the University of Missouri and were subjected to the same management and diet.

Collection of the uterine luminal flush (ULF). Fertility-classified heifers (HF, $\mathrm{n}=22$; SF, $\mathrm{n}=14 ; \mathrm{IF}, \mathrm{n}=6$ ) were synchronized to estrus (day 0 ) and received two in vivo-produced embryos on day 7. All heifers were slaughtered on day 17 (10 days post-transfer), and the uterine lumen gently flushed with $20 \mathrm{ml}$ of sterile and filtered $1 \mathrm{X}$ PBS. The conceptuses were removed, if present, the ULF clarified by centrifugation $\left(3000 \mathrm{x} g\right.$ at $4{ }^{\circ} \mathrm{C}$ for $\left.15 \mathrm{~min}\right)$, and the supernatant was carefully removed with a pipette, mixed, divided into aliquots, frozen in liquid nitrogen, and stored at $-80^{\circ} \mathrm{C}$ until analyzed. 
Glucose Analysis. Concentrations of glucose in pregnant (HF, n=16; SF, n=9; IF, n=1) and open (HF, $n=6 ; S F, n=5 ; I F, n=5)$ ULF and blood plasma were measured using the MyQubit Amplex ${ }^{\circledR}$ Red Glucose Assay with Qubit ${ }^{\circledR}$ 3.0 Fluorometer, following the manufacture instructions. The Amplex ${ }^{\circledR}$ Red/UltraRed stop reagent was used to terminate the fluorescence signal-generating reaction in the enzymatic assay, and fluorescence was measured 15 minutes after the stop reagent was added.

Eicosanoids Analysis. The assay was conducted at the Eicosanoid Core Laboratory of Vanderbilt University on ULF samples collected from pregnant (HF, $n=5 ; S F, n=5)$ and open (HF, n=5; SF, n=5; IF, n=5) heifers. To quantify PGs, a total of $100 \mathrm{uL}$ of each sample was placed in a microcentrifuge tube containing $25 \%$ methanol in water $(500 \mathrm{uL})$ and internal standard ( $\mathrm{d}_{4}-\mathrm{PGE}_{2}$ and $\mathrm{d}_{4}-\mathrm{LTB}_{4}, 1 \mathrm{ng}$ each). The sample was vortexed and then extracted on an Oasis MAX $\mu$ Elution plate (Waters Corp., Milford, MA) as follows. Sample wells were first washed with methanol (200 uL) followed by $25 \%$ methanol in water $(200 \mathrm{uL})$. The sample was then loaded into the well and washed with $600 \mathrm{uL} 25 \%$ methanol. Eicosanoids were eluted from the plate with $30 \mathrm{uL} 2-$ propanol/acetonitrile $(50 / 50, \mathrm{v} / \mathrm{v})$ containing $5 \%$ formic acid into a 96 -well elution plate containing $30 \mathrm{uL}$ water in each well.

Samples were analyzed on a Waters Xevo TQ-S micro triple quadrupole mass spectrometer connected to a Waters Acquity I-Class UPLC (Waters Corp., Milford, MA USA). Separation of analytes was obtained using an Acquity PFP column (2.1 x $100 \mathrm{~mm})$ with mobile phase A being $0.01 \%$ formic acid in water and mobile phase B acetonitrile. 
Eicosanoids were separated using a gradient elution beginning with 30\% B going to $95 \%$ B over 8 minutes at a flow rate of $0.250 \mathrm{~mL} / \mathrm{min}$.

Fatty acids Analysis. Total fatty acid analysis was conducted by high-resolution mass spectrometry at the Southeast Center for Integrated Metabolomics (SECIM) of the University of Florida on ULF samples collected from pregnant $(\mathrm{HF}, \mathrm{n}=5$; $\mathrm{SF}, \mathrm{n}=5)$ and open (HF, $\mathrm{n}=5 ; \mathrm{SF}, \mathrm{n}=5 ; \mathrm{IF}, \mathrm{n}=5)$ heifers.

Unless otherwise stated, all reagents used were of LC-MS grade and obtained from Fisher Scientific (Fairlawn, NJ). A sample of $100 \mathrm{mg}$ was weighed into a clean conical glass tube ( $5 \mathrm{~mL}$ volume) and $1 \mathrm{~mL}$ of acetonitrile containing $100 \mathrm{mg} / \mathrm{L}$ of butylated hydroxy toluene (BHT) was added, followed by the addition of $0.5 \mathrm{~mL}$ of hydrochloric acid (37\%). The sample was sonicated for $5 \mathrm{~min}$ and heated between $80-90^{\circ} \mathrm{C}$ for 2 hours in a heating block. The addition of hydrochloric acid and heating causes hydrolysis to release fatty acids from complex lipids. After cooling at room temperature, $2 \mathrm{~mL}$ of hexane was added, and the sample was centrifuged for $1 \mathrm{~min}$ at 3,260 $\mathrm{x}$. The top layer $(1.5 \mathrm{~mL})$ was removed, transferred to a clean glass culture tube, and dried under a gentle stream of nitrogen. The dried sample was reconstituted in $0.5 \mathrm{~mL}$ of $80 / 20$ acetonitrile/5 mM ammonium acetate, and $10 \mu \mathrm{L}$ of the injection standard mixture (DHA-D5, EPA-D5, $\alpha-$ LA-D14) was added to the reconstituted sample. The final sample was transferred to an LC vial for LC-MS analysis.

Fatty acid analysis was performed on a Thermo Q-Exactive Orbitrap Mass Spectrometry with Dionex Ultimate 3000 UHPLC and autosampler. Separation was achieved on a Waters HSS T3 column $(150 \times 2.1 \mathrm{~mm}, 1.8 \mu \mathrm{m})$ at a flow rate of $0.5 \mathrm{~mL} / \mathrm{min}$ and column temperature of $30 \mathrm{C}$ with mobile phase $\mathrm{A}$ and $\mathrm{B}$ consisting of $1 \mathrm{mM}$ ammonium 
acetate in water and $0.1 \%$ acetic acid in acetonitrile, respectively. Gradient elution started at $25 \% \mathrm{~A}$ and $75 \% \mathrm{~B}$ from $0-0.5$ min with a linear increase to $90 \% \mathrm{~B}$ from 0.5 to $7 \mathrm{~min}$, a further increase to $95 \%$ B from 7-8 min and then held constant at 95\% B from 8-21 min. The column was returned to initial conditions in $0.5 \mathrm{~min}$ and equilibrated for $4.5 \mathrm{~min}$. The mass spectrometry was operated in negative heated electrospray ionization (HESI) mode with a resolution setting of 70,000 at $\mathrm{m} / \mathrm{z} 200$, collecting $\mathrm{m} / \mathrm{z}$ 100-700. Data dependent analysis was conducted on the top 5 most abundant peaks throughout the run with a resolution setting of 17,500, ion time of $75 \mathrm{~ms}$ and collision induced dissociation of 30,50 and 70. The HESI settings were $3 \mathrm{kV}, 100 \mathrm{C}$ probe temperature, $300 \mathrm{C}$ capillary temperature, 40 arb sheath gas, 5 arb auxiliary gas, and 1 arb sweep gas. Fatty acids were identified by accurate mass $(<10 \mathrm{ppm}$ accuracy), retention time and tandem mass spectrometry.

Untargeted Lipidomic Analysis. Global lipidomics was conducted on ULF from open $(\mathrm{n}=5)$ and pregnant $(\mathrm{n}=5)$ HF heifers. The assay was conducted at the Southeast Center for Integrated Metabolomics (SECIM) of the University of Florida.

Samples were extracted following a cellular extraction procedure without prenormalization to the sample protein content. Global lipidomics profiling was performed on a Thermo Q-Exactive Orbitrap mass spectrometer with Dionex UHPLC and autosampler. All samples were analyzed in positive and negative heated electrospray ionization with a mass resolution of 35,000 at $\mathrm{m} / \mathrm{z} 200$ as separate injections. Separation was achieved on an Acquity BEH C18 $1.7 \mu \mathrm{m}, 100$ x $2.1 \mathrm{~mm}$ column with mobile phase A as 60:40 Acetonitrile:10 mM Ammonium formate with $0.1 \%$ formic acid in water and mobile phase B as 90:8:2 2-propanol: acetonitrile: 10mM ammonium formate with 0.1\% 
formic acid in water. The flow rate was $500 \mu \mathrm{L} / \mathrm{min}$ with a column temperature of $50^{\circ} \mathrm{C} .5$ $\mu \mathrm{L}$ was injected for negative ions and $3 \mu \mathrm{L}$ for positive ions.

Data from positive and negative ion modes were separately analyzed using LipidMatch software. First, all MS2 raw files were converted to "ms2" and MS raw files to "MzXML" using MSConvert. A peak list was generated after running MzMine on all MzXML files. An input folder that included all "ms2" files and the peak list were used to run LipidMatch to identify features.

Statistical Analyses. Statistical analyses for measurements of glucose, prostaglandins, and fatty acids were conducted using SAS (SAS Institute Inc., Cary, NC). All continuous data was assessed for normality using the UNIVARIATE procedure of SAS. Glucose concentrations in ULF were not normally distributed and were log transformed. The effect of pregnancy status (pregnant vs non-pregnant), conceptus number (1 vs 2 conceptuses), and fertility classification on glucose, prostaglandins, and fatty acids concentrations were determined by ANOVA using the GLM procedure. Post-test comparisons were conducted using the LSMEANS statement with the Fisher's protected LSD option. Pearson's correlation between glucose concentrations in plasma and in the ULF, and the correlation between conceptus size (for heifers that had only one conceptus recovered) and glucose in the ULF were determined using the CORR procedure.

For the lipidomics data, statistical analysis was performed on MetaboAnalyst 4.0 [408-410]. A table matrix of $\mathrm{m} / \mathrm{z}$ peak intensities with samples in columns and features in rows were created and imported to MetaboAnalyst 4.0, and data pre-processing consisted of autoscaling normalization [411]. Fold change (FC) analysis was conducted to identify features that increased or decreased with pregnancy, and the standard fold change threshold 
of 2 was used. A t-test was used to investigate if features were differently $(\mathrm{P}<0.05)$ abundant between pregnant and open ULF.

Integration of uterine luminal content and endometrium and conceptus transcriptome. To further investigate the biology of subfertility, we integrated the present ULF data with transcriptome data from endometrium and conceptuses which have been performed in the same group of animals and recently published [179]. The Kyoto Encyclopedia of Genes and Genomes (KEGG) database (https://www.genome.jp/kegg/) was used for identifying genes associated with glucose, prostaglandins and lipid metabolism.

To explore the relationship of endometrium transcriptome and ULF composition, we first compared data from day 17 endometrium from pregnant $(\mathrm{HF}, \mathrm{n}=5 ; \mathrm{SF}, \mathrm{n}=5)$ and open $(\mathrm{HF}, \mathrm{n}=5 ; \mathrm{SF}, \mathrm{n}=5)$ heifers, to detect differences in endometrium gene expression associated with the metabolism of glucose, PGs and lipids that are normally induced by pregnancy. Then, we compared the endometrium transcriptome of only pregnant heifers $(\mathrm{HF}, \mathrm{n}=5$ versus $\mathrm{SF}, \mathrm{n}=5)$, to explore differences in endometrium gene expression that could be associated with the detected differences in ULF composition, and possibly, related to the pregnancy loss occurring in SF heifers.

A similar approach was used to investigate the association of ULF composition and conceptus transcriptome. Because there is natural variation in conceptus length among conceptuses collected in the same day during the period of conceptus elongation in cattle $[29,36,94,328,330]$ and the conceptus transcriptome changes as it develops [94], we first analyzed day 17 conceptus transcriptome data from HF $(n=15)$ and SF heifers $(n=7)$ that were either short $(n=11$; mean length: $2.5 \pm 0.4 \mathrm{~cm})$ or long $(n=11$; mean length: $14.5 \pm$ $1.9 \mathrm{~cm}$ ) to explore differences in the transcriptome of conceptuses that were likely due to 
stage of development. Then, transcriptome of HF $(n=17)$ and SF $(n=10)$ conceptuses were compared for the same set of genes of interest, to investigate the influence of ULF composition on conceptus transcriptome, in order to explore the mechanisms associated to the retarded growth of SF conceptuses and reduced pregnancy success in SF heifers.

\section{RESULTS}

Glucose Concentrations in ULF. Glucose concentrations in ULF were higher (P $<0.01$ ) in pregnant compared to nonpregnant heifers on day 17 (Figure 1A). The number of conceptuses in the uterus of pregnant heifers did not influence $(\mathrm{P}=0.43)$ glucose concentrations in the ULF (Figure 1B). Among pregnant heifers, there was no effect $(\mathrm{P}=$ 0.19) of fertility classification on ULF glucose concentrations (Figure 1C). Furthermore, ULF glucose concentrations did not $\operatorname{differ}(\mathrm{P}=0.66)$ among open fertility-classified heifers (Figure 1D).

Circulating concentrations of glucose in plasma were not different $(\mathrm{P}=0.35)$ between pregnant and nonpregnant heifers (Figure 1E), between heifers with one versus two $(\mathrm{P}=0.90)$ conceptuses present (Figure $1 \mathrm{~F})$, or among pregnant $(\mathrm{P}=0.82)$ fertilityclassified heifers (Figure 1G). Among open animals, SF heifers had higher $(\mathrm{P}=0.02)$ plasma glucose concentrations than IF heifers (Figure 1H).

In pregnant heifers, glucose concentration in ULF was correlated (Figure 2A) with glucose concentrations in plasma $(\mathrm{r}=0.43 ; \mathrm{P}=0.03)$, but there was no correlation $(\mathrm{r}=$ $0.44 ; \mathrm{P}=0.13$ ) between conceptus size and ULF glucose concentrations (Figure 2B).

\section{Transcriptome analysis of endometrium and conceptuses for glucose transport and} metabolism genes. Expression of genes encoding glucose transporters (Dataset S1) that 
increased in pregnant compared to open endometrium (SLC2A1, SLC5A1, SLC5A11, SLC35D2 and SLC5A4) were not different (FDR $\geq 0.68$ ) between pregnant HF and SF endometrium (Table 1). Interestingly, expression of other glucose transporters (SLC2A10, SLC2A3 and SLC37A4) decreased (FDR $<0.05)$ with pregnancy, but their expression was also not different $(\mathrm{FDR} \geq 0.65)$ in pregnant endometrium of $\mathrm{HF}$ and SF heifers (Table 1).

No differences were observed in transcripts encoding glucose transporters between short (mean length: $2.5 \pm 0.4 \mathrm{~cm}$ ) and long conceptuses (mean length: $14.5 \pm 1.9 \mathrm{~cm}$ ), and the five most abundant glucose transporters in the elongating conceptuses were SLC2A1, SLC2A5, SLC37A1, SLC2A8 and SLC2A13 (Dataset S2). Interestingly, expression of SLC2A2 (GLUT2) and SLC2A4 (GLUT4) was higher in SF than HF conceptuses (Dataset S3).

For genes involved with glycolysis and gluconeogenesis according to the KEGG database, expression of 13 genes increased and 3 decreased in pregnant compared to open endometrium (Dataset S4 and Table 2), and expression of one gene (FBP1, fructosebisphosphatase 1) was increased in pregnant endometrium of HF compared to SF heifers (Dataset S5 and Table 2). None of the selected genes involved with glycolysis and gluconeogenesis were differently expressed in short vs long conceptuses (Dataset S6), but expression of HK1, FBP1 and GALM was increased in HF conceptuses, and ALDOB and ALDH3B1 was increased in SF conceptuses (Dataset S7 and Table 3).

Prostaglandins in the ULF. Pregnant heifers tended $(\mathrm{P}=0.07)$ and had $(\mathrm{P}<0.01)$ greater concentrations of PGE2 and PGF2 $\alpha$ in the ULF than nonpregnant heifers (Figures $3 \mathrm{~A}$ and $3 \mathrm{E}$, respectively). However, ULF concentrations of 6-keto-PG1 was not affected ( $\mathrm{P}=$ 0.18 ) by pregnancy status (Figure 3I), because 6-keto-PG1 concentrations did not differ 
among pregnant and open SF heifers $(\mathrm{P}=0.61$; LSMEANS open $=29.7 \pm 8.3 \mathrm{ng} / \mathrm{mL}$, pregnant $=23.5 \pm 8.3 \mathrm{ng} / \mathrm{mL}$ ), even though 6-keto-PG1 was increased by 5.4 fold in the ULF of pregnant than open HF heifers $(\mathrm{P}<0.01$; LSMEANS open $=8.5 \pm 6.4 \mathrm{ng} / \mathrm{mL}$, pregnant $=45.8 \pm 6.4 \mathrm{ng} / \mathrm{mL}$ ). Furthermore, among pregnant heifers, the number of conceptuses present in the uterus ( 1 vs 2) did not influence ULF concentrations of PGE2 $(\mathrm{P}=0.39$; Figure 3B $), \mathrm{PGF} 2 \alpha(\mathrm{P}=0.14$; Figure $3 \mathrm{~F})$, and 6-keto-PG1 $\quad(\mathrm{P}=0.50$; Figure 3J). Similarly, ULF PG concentrations were not different $(\mathrm{P} \geq 0.32)$ among open fertilityclassified heifers (Figures 3D, 3H and 3L). Interestingly among pregnant heifers, ULF concentrations of PGE2, PGF2 $\alpha$, and 6-keto-PG1 tended $(\mathrm{P} \leq 0.10)$ to be higher in HF compared to SF heifers (Figures 3C, 3G and 3K).

Transcriptome analysis of endometrium and conceptuses for PG signaling, arachidonic acid metabolism and PPAR signaling genes. Expression of selected genes involved with PG signaling by day 17 endometrium and conceptuses are summarized in Table 4. Expression of selected genes encoding PG synthases did not differ in the endometrium of pregnant HF vs SF heifers, or between conceptuses collected from HF or SF heifers. Endometrium expression of PTGR1, PTGR3, PTGFR, PTGIR and PPARG was very low $($ FPKM $<1)$. Among conceptuses, PGF receptor (PTGFR) expression was higher in HF than SF conceptuses, and PTGFR was the overall highest PG receptor expressed by the conceptuses. Conceptus expression of PTGER1, PTGER2, PTGER3, PTGER4, PTGIR and PPARA was also very low (FPKM $<1)$. Additionally, the overall expression of PPAR receptors by the endometrium or conceptuses did not differ in all comparisons. Interestingly, the expression of the PG transporter SLCO2A1 was upregulated in HF than 
SF conceptuses, but no differences were observed among short and long conceptuses, or in the endometrium (Table 4).

Expression of genes involved with arachidonic acid metabolism differed between pregnant and open endometrium. Expression of seven genes were increased in open endometrium (CYP2C18, ALOX15B, PTGDS, PTGIS, PLA2G4B, ALOX15 and CYP2U1), and 10 genes increased pregnant endometrium (CYP2J2, PLA1A, ALOX12, CBR3, PLA2G3, HPGDS, LOC506594 (prostaglandin F synthase 1-like), PTGES3, CBR1 and PLA2G2A-2) (Dataset S8). However, none of the genes associated with arachidonic acid metabolism were differently expressed in endometrium of pregnant HF and SF heifers (Dataset S9). In conceptuses, expression of only one gene (LOC506594; prostaglandin F synthase 1-like) was increased in short compared to long conceptuses (Dataset S10), and expression of one gene (PLA2G15) increased and three (PLA2G1B, GPX1 and CBR1) decreased in HF compared to SF conceptuses (Dataset S11).

Among genes involved with PPAR signaling, expression of 11 genes (PCK2, PLIN2, ACSL5, FABP3, SCD, OLR1, UBC, SLC27A5, FADS2, SLC27A6 and ACSL4) increased and 5 decreased (FABP7, PLTP, SCD5, RXRA and CPT1C) in pregnant compared to open endometrium (Dataset S12). However, expression of only one gene differed between pregnant $\mathrm{HF}$ vs $\mathrm{SF}$ endometrium, as expression of FABP3 was increased by 2-fold in the endometrium of pregnant HF heifers (Dataset S13).

In conceptuses, expression of one gene (FADS2) in the PPAR signaling pathway increased in long compared to short conceptuses (Dataset S14), and expression of one gene increased (PDPK1) and 6 decreased (FABP3, APOA5, SORBS1, ACADL, LPL and ACADM) in HF compared to SF conceptuses (Dataset S15). 
Fatty Acids in the ULF. A total of 37 fatty acids were identified in the ULF of the fertilityclassified heifers, and their concentrations in pregnant and open ULF is presented in Figure 4A. Two saturated fatty acids, palmitic acid (16:0) and stearic acid (18:0), were the predominant fatty acids detected in the ULF of both open and pregnant heifers (Figure 4A), accounting for about $90 \%$ of the total fatty acid content detected in the ULF (Figures 4B and 4C). The percentage of the subsequent most predominant fatty acids in the ULF relative to total fatty acid content for both open and pregnant heifers were: $3.1 \%$ octacosaheptaenoic (28:7); 1.9\% octacosaoctaenoic acid (28:8); $1.2 \%$ arachidate (20:0); $0.8 \%$ Behenate (22:0) and 18:1;0.7\% myristate (14:0); and 0.6\% margaric (17:0) (Figures $4 \mathrm{~B}$ and $4 \mathrm{C})$. The remaining 28 fatty acids together accounted for only around $1.1 \%$ of the total fatty acid detected in ULF (Dataset S16). A heatmap for fatty acid concentration in the ULF across samples is presented in Figure 5, and surprisingly, there was no difference in fatty acid content between ULF from open and pregnant heifers. When HF and SF heifers were analyzed separately, the fatty acid content that were influenced by pregnancy was not consistent in HF and SF ULF (Table 5). In comparisons of only pregnant HF and SF heifers, concentrations of 8 fatty acids increased and one decreased in the ULF of HF compared to SF heifers (Table 5). Among open HF, SF and IF heifers, concentrations of three fatty acids were increased in the ULF of HF compared to SF heifers, and two fatty acids increased in the ULF of HF compared to IF heifers (Table 5).

\section{Transcriptome analysis of endometrium and conceptuses for lipid metabolism genes.}

Expression of 66 genes associated with lipid metabolism differed between pregnant and open endometrium, with 41 genes increased and 25 decreased in the pregnant endometrium (Dataset S17). Of note, expression of genes regulating fatty acid biosynthesis (FASN, 
FADS2, ELOVL1, and ELOVL6) and fatty acid transport (SLC27A5, SLC27A6, FABP3, and LOC100299715) were upregulated in the endometrium by pregnancy. However, expression of only three genes differed in the endometrium of pregnant HF and SF heifers, as FABP3, ST6GAL2 and GALNT16 were increased in the endometrium of HF heifers (Dataset S18).

In conceptuses, expression of six genes associated with lipid metabolism differ between short and long conceptuses (Dataset S19). Expression of ELOVL7 and INPP4B was increased in short conceptuses, and expression of FADS1, FADS2, ELOVL5 and B3GALT5 was increased in long conceptuses. Interestingly, expression of 24 genes differ between HF and SF conceptuses (Dataset S20). Expression of ten genes (ELOVL5, FADS1, GPAM, CHKA, CHSY1, CYP11A1, PI4KB, B3GALT5, XYLT2, and B3GNT5) were increased in HF conceptuses, and 14 genes (ELOVL7, FABP3, SLC27A3, INPP4B, ASAH1, B4GALT4, GALNT5, ACAT1, CEPT1, PIGN, NDST3, ITPKA, ST6GAL2, and CERS1) increased in SF conceptuses.

Untargeted Lipidomics. There was a total of 17,480 features detected from the positive mode (Dataset S21) and 2,020 features detected in the negative mode (Dataset S22). Only features identified based on fragmentation spectra (ddMS2) was subjected to statistical analysis, which represents 22 features detected in the positive ion mode.

Fold change analysis found that abundance of nine lipids increased and one decreased (Figure S1) in the uterine lumen by pregnancy, and 5 of the 9 lipids which increased by pregnancy in the FC analysis were significantly different in the t-test (Table 6). The differently abundant lipids included phosphatidylcholines, triacylglycerides and acylcarnitines. 


\section{DISCUSSION}

The present study was conducted to investigate uterine luminal concentrations of glucose, prostaglandins and lipids in fertility-classified heifers to gain insights in the mechanisms regulating uterine receptivity. Conceptus elongation is characterized by rapid proliferation of trophectoderm cells, therefore sufficient energy and anabolic precursors are required to support cellular replication and specialization [153]. In mammalian embryos, at the cleavage stage, ATP production is based on low levels of oxidation of pyruvate, lactate, and amino acids, and by the blastocyst stage, energy demands increase dramatically with the formation of the blastocoel and due to the increase in protein synthesis required for conceptus growth $[412,413]$. The blastocyst preferably uses glucose as energy source compared to embryos in earlier stages of development, and rather than oxidize the glucose consumed, aerobic glycolysis seems to be preferred pathway, which is a metabolic adaptation of rapidly proliferative cells known as Warburg effect [140, 154, 157]. This same pattern of glucose metabolism was observed during conceptus elongation in sheep [414]. Thus, glucose availability in the uterine lumen and the expression of glucose transporters by the elongating conceptus are essential for pregnancy success in ruminants.

Gluconeogenesis appears not to occur in the uterus of mice and humans, because all the necessary enzymes required for the synthesis of glucose from non-carbohydrate carbon substrates are not expressed in the uterus. The gluconeogenic enzyme phosphoenolpyruvate carboxykinase (PEPCK, also known as PCK), which converts oxaloacetate into phosphoenolpyruvate and carbon dioxide, was not detected in mouse uterus [144], and PCK and fructose-1,6-bisphosphatase (FBP1), which converts fructose- 
1,6-bisphosphate to fructose 6-phosphate, were also not detected in human myometrium and endometrium [145]. In the present study, the expression of rate-limiting gluconeogenic enzymes (G6PC, PCK2, FBP1) increased in pregnant compared to open endometrium. Similarly, mRNA expression of those same 3 enzymes were found to increase in the intercaruncular endometrium from day 28 to 42 of gestation in cattle [146], indicating that perhaps the bovine uterus is able to generate glucose through gluconeogenesis, and that this gluconeogenic capacity of the uterus increases as pregnancy advances. Nonetheless, the uptake of glucose into the uterine lumen through glucose transporters expressed by the endometrium appears to be the main pathway regulating uterine luminal concentrations of glucose in ruminants [138]. In fact, concentrations of glucose in the uterine lumen of ewes increased by 6-fold from days 10 to 15 of pregnancy, which occurred concomitant with the increase in expression of glucose transporters (SLC2A1, SLC5A1, SLC5A11) by the endometrium $[138,139]$. These glucose transporters are non-classical IFNT-stimulated genes [108] and were also found to be increased by pregnancy in the endometrium in the present study, thus likely contributed to the higher uterine luminal concentrations of glucose observed in the ULF of pregnant compared to open heifers. The moderate positive correlation observed between plasma and uterine luminal concentrations of glucose perhaps demonstrates the active transport of glucose from plasma into the uterine lumen through the up-regulated glucose transporters. Nevertheless, no differences in uterine luminal concentrations of glucose were observed between pregnant HF and SF heifers, which was supported by the endometrium transcriptome data, as only one gene (FBP1) involved with gluconeogenesis was increased in pregnant $\mathrm{HF}$ than SF endometrium. These findings indicated that the availability of glucose in the ULF is probably not a major factor influencing subfertility in this group of fertility-classified heifers. Although no differences 
were observed between short and long conceptuses for genes encoding glucose transporters, expression of SLC2A2 (GLUT2) and SLC2A4 (GLUT4) were higher in SF than HF conceptuses, which could indicate a dysregulated energy metabolism in SF conceptuses, as 5 genes involved with glycolysis and gluconeogenesis (HK1, FBP1, GALM, ALDOB, ALDH3B1) were differently expressed between HF and SF conceptuses.

Prostaglandins are major regulators of female reproductive function $[415,416]$ and modulate conceptus and endometrial gene expression during early pregnancy in ruminants [120]. In fact, PTGS2 was a key player regulating overall gene expression of day 17 bovine conceptuses [179]. In the present study, uterine luminal concentrations of PGE2, PGF2 $\alpha$, and 6-keto-PG1 $\alpha$ tended to be higher in the ULF of pregnant HF than SF heifers. Although no differences in endometrium and conceptus mRNA expression of selected prostaglandin synthases (PTGS2, PTGIS, PTGES, PTGFS) were observed, the increase in uterine luminal concentrations of PGs in HF heifers is likely due to differences in conceptusderived PGs, as HF heifers had longer conceptuses and therefore a larger number of cells producing PGs. Likewise, an earlier study observed no differences in IFNT mRNA between bovine conceptuses that were either long $(>10 \mathrm{~cm})$ or short $(<5 \mathrm{~cm})$, but IFNT protein was substantially increased in the uterine flush from long conceptuses [109].

Prostaglandin E2, PGF2 $\alpha$, and PGI2 signals through their designated receptors, termed EP, FP, and IP, respectively. There are 4 subtype of PGE2 receptors (EP1, EP2, EP3, and EP4) but only one PGF2 $\alpha$ receptor (FP, also known as PTGFR) and one PGI2 receptor (IP, also known as PTGIR) [209, 210]. Among the EP receptors, EP2 and EP4 had the highest expression in the endometrium of open and pregnant cows. These receptors are members of the seven-transmembrane $G$ protein-coupled receptors which signals through cAMP [417], and the PGE2-cAMP pathway regulates important biological processes such 
as angiogenesis, vasodilatation, myometrial quiescence, and decidualization, which are essential for pregnancy establishment in several species [231]. The luteotropic effects of PGE2 are well described [418], and it has been recently reported that intrauterine infusions of low doses of PGE1 blocked the luteolytic effects induced by intrauterine infusions of PGF2 $\alpha$ in cattle [419]. Because PGE1 and PGE2 acts through the same EP receptors [420], it is possible that the reduced uterine luminal concentration of PGE2 in pregnant SF heifers resulted in diminished luteotropic effects on the corpus luteum (CL), which might have contributed to the higher pregnancy loss in SF heifers.

In the present experiment, the expression of PGF synthase (PTGFS) by day 17 conceptuses was low, but the expression of the PGF2 $\alpha$ receptor (PTGFR) was the highest among the PG receptors investigated in the conceptuses, suggesting that paracrine actions of endometrial PGF2 $\alpha$ might be of particular importance regulating bovine conceptus elongation, and interestingly, HF conceptuses expressed higher PTGFR than SF conceptuses. Although the effect of PGF2 $\alpha$ on embryonic and trophectoderm cell growth have not been investigated, PGF2 $\alpha$ has been shown to induce endometrial cell proliferation, angiogenesis, tissue remodeling, and to regulate the expression of genes involved with conceptus endometrium interactions [226, 421] which are important biological events occurring during conceptus elongation. Furthermore, higher expression of a PG transporter (SLCO2A1) was observed in HF than SF conceptuses, which could be a result of higher PGs available in the ULF of pregnant HF heifers, and may indicate higher PG signaling in HF conceptuses. The solute carrier organic anion transporter family member 2A1 (SLCO2A; also known as PGT) [422] is able to transport PGE1, PGE2, PGD2, PGF2 $\alpha$ and, to a lesser extent TXB2 [423]. Although newly synthesized PGs may exit the cells by diffusion due to their lipophilic nature, SLCO2A1 is involved in the 
transport of PGs across membranes, and plays important role particularly mediating the inactivation of the PG stimulus, which depends on the uptake of the PG molecule signaling through membrane bound receptors into the cytoplasm of the cell, where it is oxidized by 15-ketoprostaglandin dehydrogenase (15-PGDH) [424, 425]. The expression of another transmembrane PG transporter, the ATP-binding cassette, subfamily C member 4 (ABCC4; also known as MRP4) [426], which is expressed by the pig endometrium and conceptus [427] was not detected on day 17 endometrium or conceptus in the present study.

High uterine luminal concentrations of 6-keto-PG1 was detected in open or pregnant heifers, and PGI2 synthase was highly expressed by the endometrium in pregnant and open heifers and by conceptuses. Although the expression of PGI2 synthase and PGI2 receptors by the endometrium and conceptus during the time of maternal recognition of pregnancy have been studied in sheep [428], the downstream effects of PGI2 signaling in the endometrium and conceptus is poorly understood. PGI2 is a strong vasodilator and a potent platelet inhibitor [429, 430]. Additionally, PGI2 is essential for implantation and decidualization in mice [214], which is an event mediated particularly through the nuclear receptor PPARD. PGI2 signaling through PPARD in sheep conceptuses does not seem to be important, as knock down of PPARD in trophectoderm cells using morpholino antisense oligonucleotides (MAO) did not disrupt conceptus elongation [431]. In contrast, the knock down of PPARG using MAO resulted in severely growth-retarded conceptuses [431] indicating that PPARG signaling is of great importance during conceptus elongation in ruminants. Furthermore, PPARG expression has been reported to increase during conceptus elongation in cattle, and its expression was correlated with the expression of several genes involved in lipid metabolism [94]. Of note, PPARA which regulates FA catabolism in several tissues [432], was the highest PPAR expressed by day 17 
endometrium, and perhaps have critical roles modulating uterine luminal lipid content during early pregnancy.

Among genes involved with PPAR signaling in the endometrium, the expression of fatty acid binding protein 3 (FABP3) was increased in pregnant compared to open heifers, and in HF compared to SF heifers. FABP coordinates cellular lipid responses, and reversely binds a variety of lipids, including eicosanoids and saturated and unsaturated long-chain fatty acids [433]. FABP3 modulates cell growth and proliferation [434] and is upregulated by pregnancy in the endometrium LE on days 15 and 18 of pregnancy in cattle [198].

Regarding the DEG involved with arachidonic acid metabolism, expression of glutathione peroxidase 1 (GPX1), a major antioxidant enzyme [435], was increased in SF than HF conceptuses, and may indicate greater oxidative stress in SF conceptuses. Furthermore, expression of one phospholipase A2 (PLA2) was increased in HF conceptuses (PLA2G15) and another one (PLA2G1B) increased in SF conceptuses. The superfamily of PLA2 enzymes are characterized by their ability to hydrolyze fatty acids from the sn-2 position of glycerophospholipids producing a variety of free fatty acids and lysoglycerophospholipids [436]. PLA2G15 is localized in lysosomes and hydrolyses preferentially zwitterionic phospholipids (phosphatidylcholine - PC and phosphatidylethanolamine - PE) [436, 437], and PLA2G1B is described as a pancreatic lipase [436] expressed primarily by acinar cells, and in a much lesser extent by the lungs and kidney, and has higher affinity for anionic phospholipids (phosphatidic acid - PA, phosphatidylserine - PS, phosphatidylglycerol - PG) than PC [438, 439]. This difference in PLA2 expression by HF and SF conceptuses perhaps contributed to the observed differences in ULF fatty acid composition between pregnant HF and SF heifers. 
Surprisingly, day 17 ULF fatty acid content of pregnant and open heifers did not differ. Palmitic acid (16:0) and stearic acid (18:0) are the two most abundant saturated fatty acids in mammalian cells $[188,404]$, and together, accounted for around $90 \%$ of the total fatty acid detected in the ULF. Two unusual ultra long-chain fatty acids $(C \geq 26)$ were the third (28:7) and fourth (28:8) most abundant fatty acids detected in the ULF of open and pregnant heifers. Ultra long-chain polyunsaturated fatty acids (PUFAs) have also been found in the other mammalian tissues, such as brain, retina, skin, and testis [188, 440], and in the testis, 28:5 and 30:5 are essential for sperm maturation and male fertility [441].

A number of long-chain (C11-20) and very long-chain (C21-25) fatty acids were increased in ULF of pregnant HF than SF heifers, although the differently abundant fatty acids had overall low abundance in the ULF, accounting together for only $1.2 \%$ of the total fatty acid detected. The source of long-chain saturated fatty acids in animals comes from the diet or from fatty acid de novo synthesis, which is mediated by FASN [184, 442]. Expression of FASN by the endometrium was increased by pregnancy, but it did not differ in the endometrium of pregnant HF and SF heifers, or between HF and SF conceptuses. Fatty acid biosynthesis and catabolism involves dynamic processes in which the number of carbons and double bonds change under the influence of elongation, desaturation and boxidation reactions [188]. Fatty acid extension depend on elongases (ELOVL), which are enzymes that catalyzes carbon chain extension [443]. In the present study, ELOVL1 and ELOVL6 were upregulated in the endometrium by pregnancy. In addition, ELOVL5 was upregulated in HF conceptuses, and ELOVL7 was upregulated in SF conceptuses. Fatty acid desaturase 1 and 2 (FADS1 and FADS2) are essential enzymes for PUFA biosynthesis [444, 445], and expression of FADS1 was increased in HF and long conceptuses and decreased in SF and short conceptuses. Furthermore, FADS2 expression was increased in 
long conceptuses and in pregnant endometrium. Moreover, a number of genes involved with lipid metabolism were differently expressed in HF and SF conceptuses, including genes involved with the biosynthesis of glycerolipids (GPAM) [446], phosphatidylcholines (CHKA) [447], ceramides (CERS1, ASAH1) [448, 449], steroid hormones (CYP11A1) [450], and with cholesterol esterification (ACAT1) [451]. The observed differences in lipid metabolism among HF and SF conceptuses likely contributed to the observed differences in fatty acid content in ULF among pregnant fertility-classified heifers.

PCs were the main lipid class that increased in the uterine lumen with pregnancy, which is possibly related to the secretion of extracellular vesicles by the elongating conceptus [201, 405], as PC are a major component of the cell membrane in eukaryotes [452]. Although, PC have been reported as the most abundant phospholipid in sheep endometrium on days 3,12 and 15 of the estrus cycle and pregnancy [453], and the main lipid component of uterine epithelial cells during the peri-implantation period in mice [454], pregnancy likely inhibits endometrium derived extracellular vesicles through interferon stimulated gene 15 (ISG15) signaling [455], thus conceptus derived EVs are likely responsible for the increased abundance of PC observed in pregnant ULF.

In summary, the current study describes the concentrations of glucose, PGs and lipids in the uterine lumen of fertility-classified heifers during the time of maternal recognition of pregnancy. The observed differences in the metabolism of glucose, PG and lipids by the conceptus and endometrium from fertility-classified heifers, further reinforces our hypothesis of dysregulated conceptus-endometrium interactions in SF heifers, which consequently affects the uterine luminal contents and imparts conceptus survival and elongation. 


\section{ACKNOWLEDGEMENTS}

The authors greatly appreciate the help of Dr. William R. Lamberson with statistical analysis, Kenneth Ladyman for his help caring for the animals, and Rick Disselhorst for coordinating the animal slaughter. This work was supported by NIH Grant 1 R01 HD072898 from the Eunice Kennedy Shriver National Institute of Child Health and Human Development. 


\section{FIGURES}

Glucose in ULF

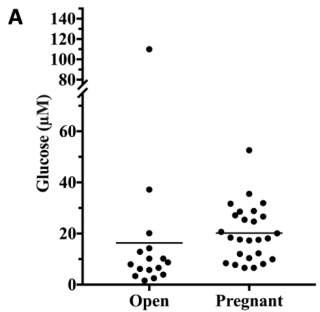

c

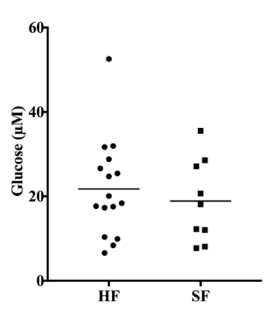

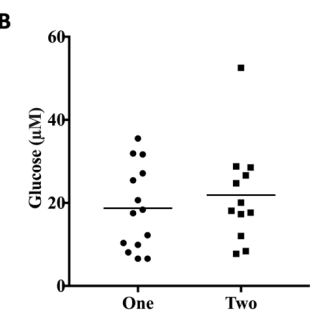

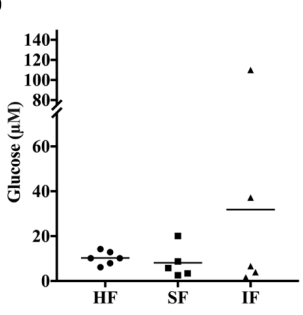

Glucose in Plasma

E

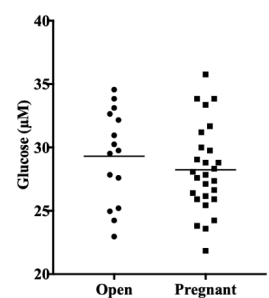

G

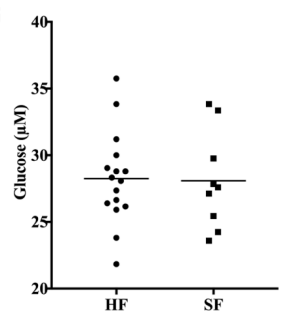

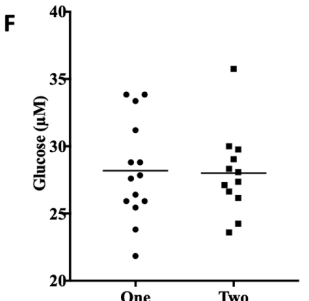

H

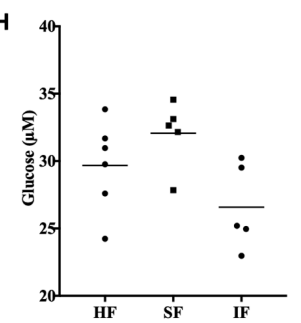

Figure 1. Glucose concentrations in the ULF and plasma of fertility classified heifers. Glucose in the uterine luminal fluid (ULF) and plasma according to pregnancy status (1A and 1E), among pregnant heifers with one or two conceptuses present in the uterine lumen (1B and 1F), among pregnant fertility-classified heifers (1C and 1G), and among open fertility-classified heifers (1D and 1H). Statistical significance was only observed in two comparisons: (1A) Concentrations of glucose increased $(\mathrm{P}<0.01)$ in pregnant than open ULF. (1H) Plasma glucose concentrations were higher $(\mathrm{P}=0.02)$ in SF than IF heifers. 
A

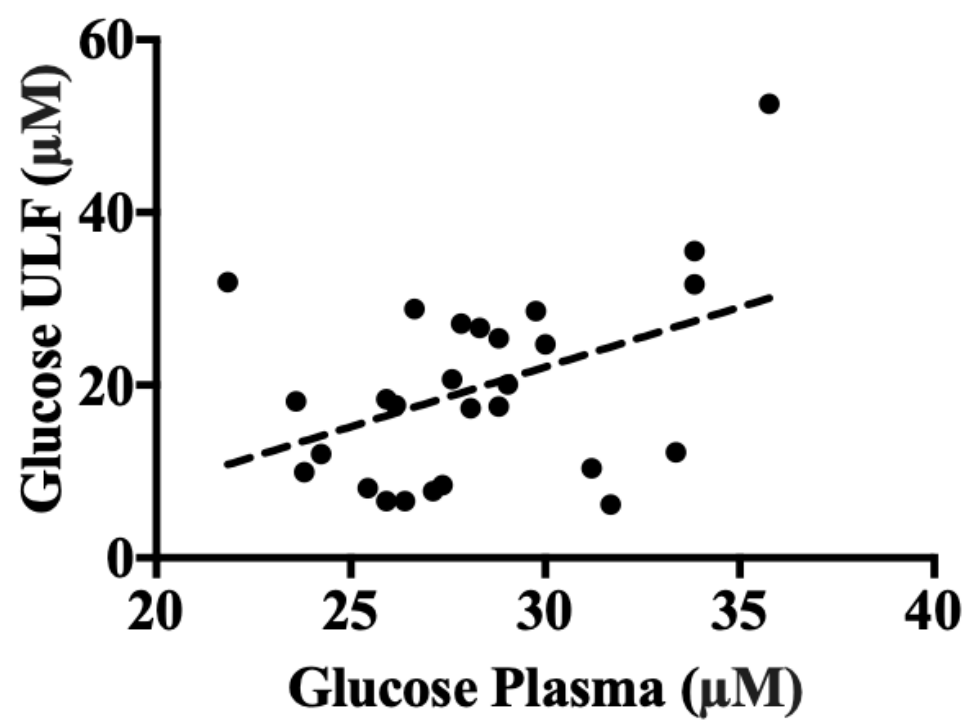

B

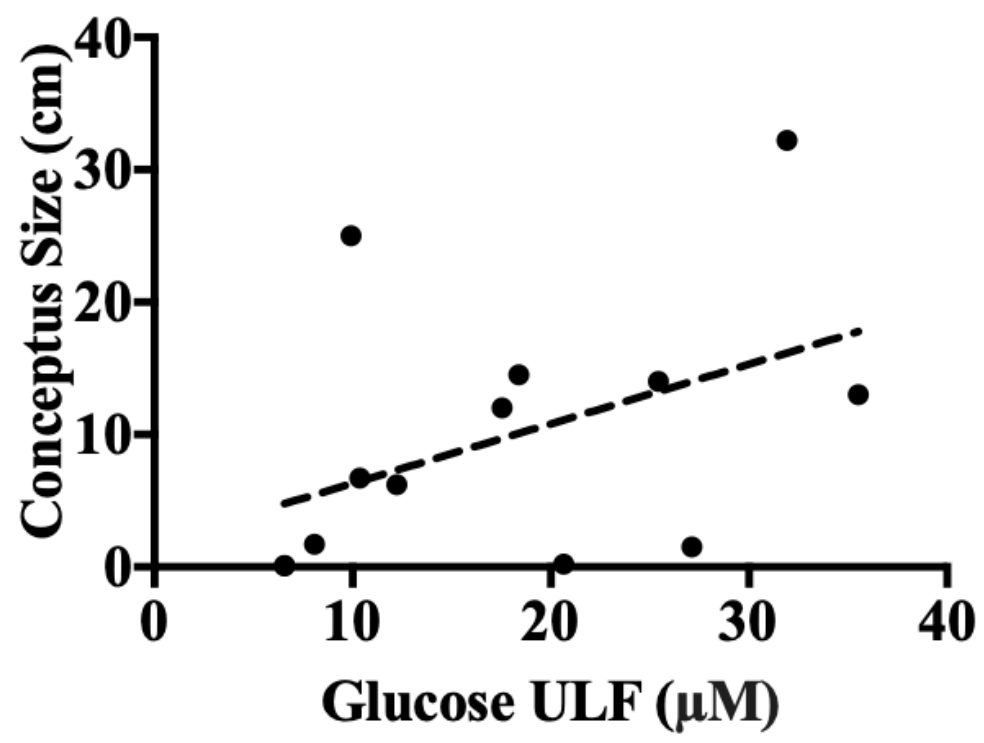

Figure 2. Correlations between ULF glucose, plasma glucose and conceptus size.

Pearson's correlation between glucose concentrations in plasma and in ULF $(r=0.43 ; \mathrm{P}=$ 0.03) (A), and correlation between conceptus size and ULF glucose $(r=0.44 ; P=0.13)$ (B). 

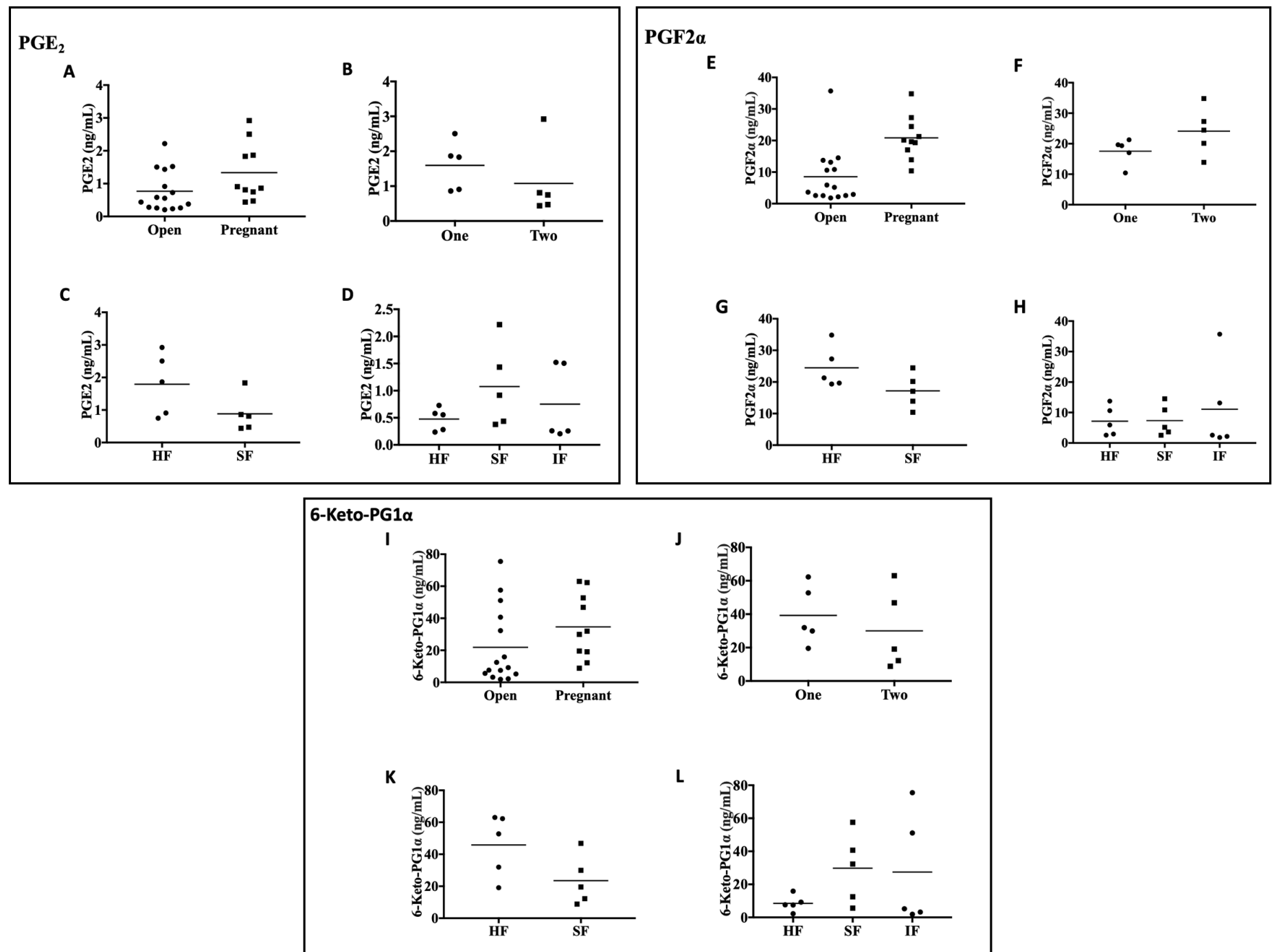

3 Figure 3. Concentrations of prostaglandins in the ULF of fertility-classified heifers. 

heifers with one or two conceptuses present in the uterine lumen (3B, 3F, and 3I), among pregnant fertility-classified heifers

$6(\mathbf{3 C}, \mathbf{3 G}$, and $\mathbf{3 K})$, and among open fertility-classified heifers (3D, 3H, and 3L). 
A

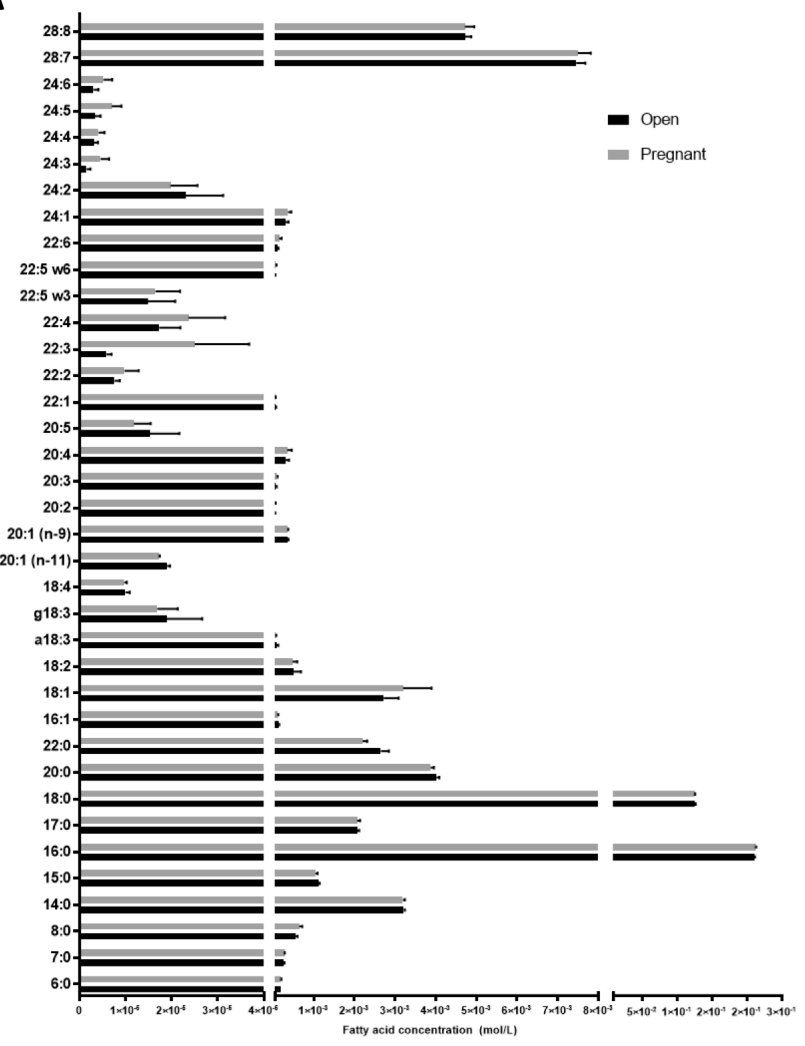

B

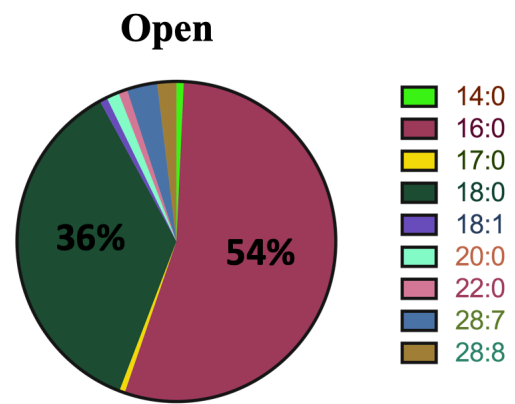

C Pregnant

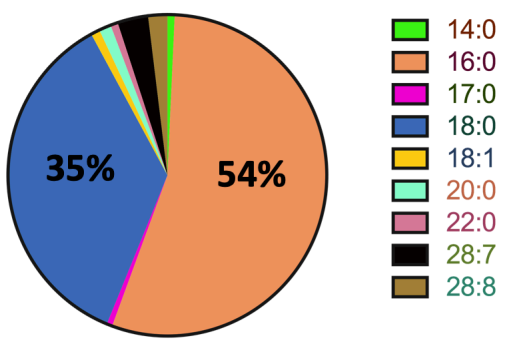

10 Figure 4. Fatty acids in ULF.

11 Concentrations of fatty acids identified in the ULF of the fertility-classified heifers (A),

12 and the proportion of the nine most abundant fatty acids in the ULF relative to the total

13 fatty acid content detected, for open (B) and pregnant (C) fertility-classified heifers. 


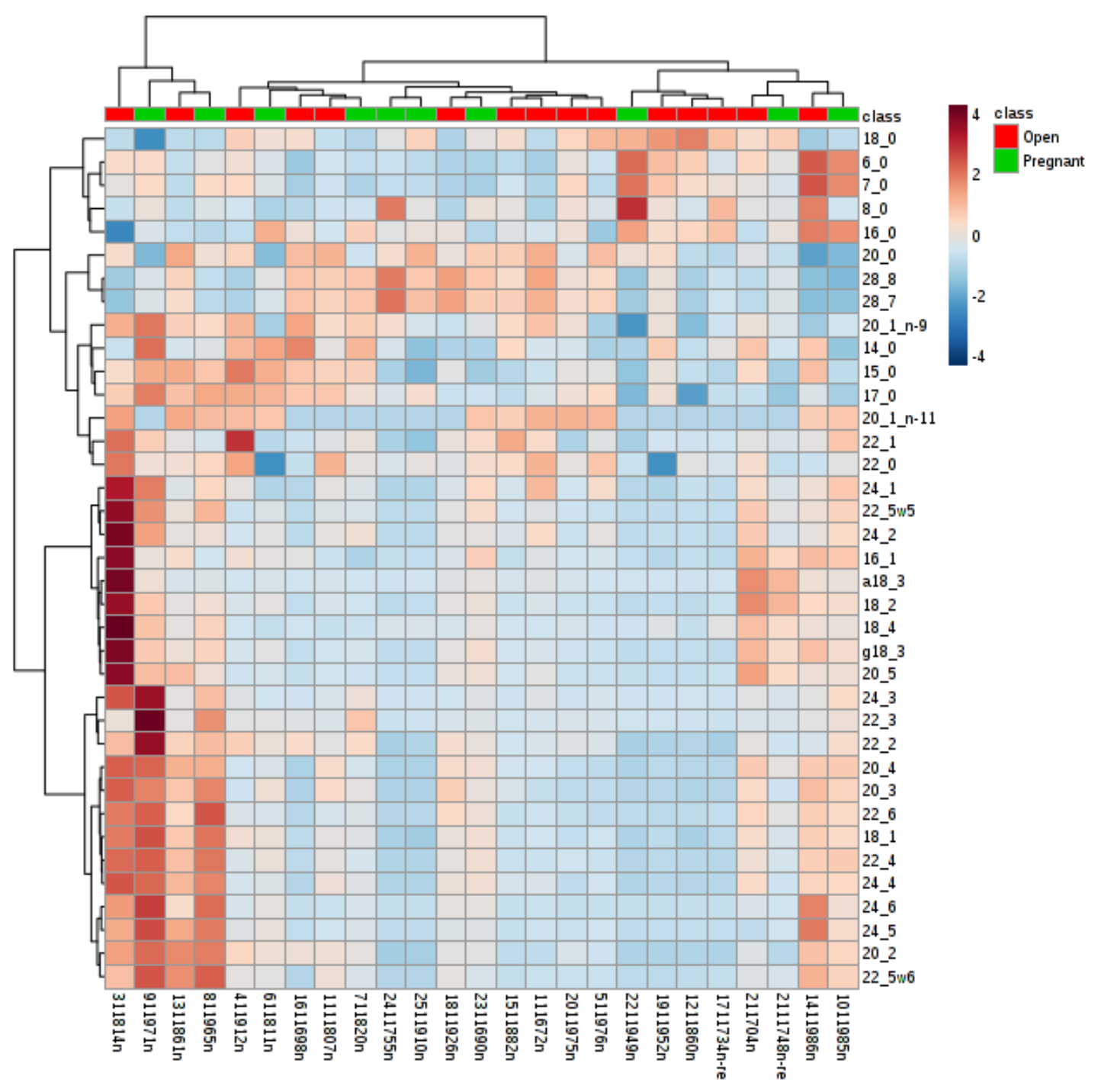

17 Figure 5. Heatmap for fatty acid concentration in the ULF across samples. 


\section{TABLES}

Table 1. Expression of genes in the endometrium involved with glucose transport

\begin{tabular}{|c|c|c|c|c|c|c|c|c|}
\hline & \multirow[b]{2}{*}{ Gene name } & \multirow[b]{2}{*}{ Gene description } & \multicolumn{3}{|c|}{ Pregnant vs Open (HF and SF) } & \multicolumn{3}{|c|}{ Only Pregnant HF vs SF } \\
\hline & & & $\begin{array}{l}\text { FPKM }^{1} \\
\text { OPEN }\end{array}$ & $\begin{array}{l}\text { FPKM }^{1} \\
\text { Pregnant }\end{array}$ & FDR & $\begin{array}{l}\text { FPKM }^{1} \\
\mathrm{HF}(\mathrm{P})\end{array}$ & $\begin{array}{l}\text { FPKM }^{1} \\
\text { SF }(P)\end{array}$ & FDR \\
\hline & SLC2A1 & Solute carrier family 2 (facilitated glucose transporter), member 1 & $68 \pm 11$ & $153 \pm 19$ & 0.00 & $174 \pm 35$ & $132 \pm 13$ & 0.68 \\
\hline $\begin{array}{l}\text { Increased in } \\
\text { Pregnant }\end{array}$ & SLC5A1 & Solute carrier family 5 (sodium/glucose cotransporter), member 1 & $21 \pm 3$ & $35 \pm 3$ & 0.00 & $31 \pm 5$ & $38 \pm 4$ & 0.90 \\
\hline \multirow[t]{2}{*}{ Endometrium } & SLC5A11 & Solute carrier family 5 (sodium/inositol cotransporter), member 11 & $0.02 \pm 0.1$ & $1 \pm 0.1$ & 0.02 & $1.2 \pm 0.3$ & $0.9 \pm 0.1$ & 0.94 \\
\hline & SLC35D2 & $\begin{array}{l}\text { Solute carrier family } 35 \text { (UDP-GlcNAc/UDP-glucose transporter), } \\
\text { member D2 }\end{array}$ & $12 \pm 1$ & $15 \pm 1$ & 0.04 & $15 \pm 1$ & $15 \pm 1$ & 1.00 \\
\hline $\begin{array}{l}\text { Decreased in } \\
\text { Pregnant }\end{array}$ & SLC2A 10 & Solute carrier family 2 (facilitated glucose transporter), member 10 & $1 \pm 0.1$ & $0.5 \pm 0.1$ & 0.00 & $0.6 \pm 0.1$ & $0.5 \pm 0.0$ & 1.00 \\
\hline \multirow[t]{2}{*}{ Endometrium } & SLC2A3 & Solute carrier family 2 (facilitated glucose transporter), member 3 & $8 \pm 0.5$ & $5 \pm 1$ & 0.00 & $5 \pm 1$ & $6 \pm 1$ & 0.65 \\
\hline & SLC37A4 & Solute carrier family 37 (glucose-6-phosphate transporter), member 4 & $7 \pm 0.2$ & $6 \pm 0.2$ & 0.04 & $5 \pm 0.3$ & $6 \pm 0.4$ & 0.88 \\
\hline
\end{tabular}

${ }^{1}$ Data is presented as fragments per kilobase of transcript per million mapped reads (FPKM) \pm standard error of the mean (SEM). 
Table 2. Differently expressed genes in the endometrium of fertility-classified heifers for genes involved with glycolysis and gluconeogenesis using KEGG database

\begin{tabular}{|c|c|c|c|c|c|}
\hline Comparisons & Gene name & Gene description & FPKM $^{1}$ & FPKM $^{1}$ & FDR \\
\hline Pregnant vs Open & & & Open & Preg & \\
\hline \multirow{7}{*}{ Increased in Open } & LDHB & Lactate dehydrogenase B & $125 \pm 5$ & $101 \pm 2$ & 0.04 \\
\hline & LDHA & Lactate dehydrogenase A & $21 \pm 2$ & $13 \pm 1$ & 0.00 \\
\hline & PFKM & Phosphofructokinase, muscle & $8 \pm 0.4$ & $5 \pm 0.3$ & 0.00 \\
\hline & HK2 & Hexokinase 2 & $1 \pm 0.3$ & $2 \pm 0.2$ & 0.01 \\
\hline & ALDOB & Aldolase, fructose-bisphosphate B & $1 \pm 0.1$ & $2 \pm 0.4$ & 0.00 \\
\hline & ADPGK & ADP-dependent glucokinase & $9 \pm 0.3$ & $11 \pm 0.5$ & 0.00 \\
\hline & PCK2 & Phosphoenolpyruvate carboxykinase 2 (mitochondrial) & $2 \pm 0.1$ & $5 \pm 0.4$ & 0.00 \\
\hline \multirow[t]{8}{*}{ Increased in Pregnant } & PFKP & Phosphofructokinase, platelet & $11 \pm 0.4$ & $16 \pm 0.9$ & 0.00 \\
\hline & FBP1 & Fructose-bisphosphatase 1 & $4 \pm 0.4$ & $12 \pm 3.7$ & 0.02 \\
\hline & HKDC1 & Hexokinase domain containing 1 & $12 \pm 2$ & $22 \pm 3$ & 0.00 \\
\hline & ACSS2 & Acyl-CoA synthetase short-chain family member 2 & $12 \pm 1$ & $23 \pm 2$ & 0.00 \\
\hline & AKR1A1 & Aldo-keto reductase family 1, member A1 & $30 \pm 1$ & $44 \pm 5$ & 0.00 \\
\hline & ENO1 & Enolase 1, (alpha) & $95 \pm 2$ & $110 \pm 5$ & 0.01 \\
\hline & PGK1 & Phosphoglycerate kinase 1 & $61 \pm 2$ & $85 \pm 5$ & 0.00 \\
\hline & ACSS1 & Acyl-CoA synthetase short-chain family member 1 & $71 \pm 7$ & $106 \pm 4$ & 0.00 \\
\hline Only Pregnant & & & SF & $\mathrm{HF}$ & \\
\hline HF vs SF & FBP1 & Fructose-bisphosphatase 1 & $7 \pm 4$ & $16 \pm 6$ & 0.00 \\
\hline
\end{tabular}


Table 3. Expression of genes involved with glycolysis and gluconeogenesis in conceptuses from fertility-classified heifers using the KEGG database

\begin{tabular}{|c|c|c|c|c|c|}
\hline Comparisons & Gene name & Gene description & FPKM $^{1}$ & FPKM $^{1}$ & FDR \\
\hline & & & Short conceptus & Long conceptus & \\
\hline Short vs Long & - & - & - & - & - \\
\hline HF vs SF Conceptuses & & & SF conceptus & HF conceptus & \\
\hline \multirow[t]{3}{*}{ Increased in $\mathrm{HF}$} & HK1 & Hexokinase 1 & $9 \pm 1$ & $19 \pm 3$ & 0.01 \\
\hline & FBP1 & Fructose-bisphosphatase 1 & $0.4 \pm 0.1$ & $1.4 \pm 0.4$ & 0.02 \\
\hline & GALM & Galactose mutarotase (aldose 1-epimerase) & $69 \pm 14$ & $207 \pm 51$ & 0.05 \\
\hline \multirow[t]{2}{*}{ Increased in SF } & ALDOB & Aldolase, fructose-bisphosphate B & $1.2 \pm 0.4$ & $0.4 \pm 0.1$ & 0.04 \\
\hline & ALDH3B1 & Aldehyde dehydrogenase 3 family member B1 & $2 \pm 0.2$ & $1 \pm 0.1$ & 0.04 \\
\hline
\end{tabular}

${ }^{1}$ Data is presented as fragments per kilobase of transcript per million mapped reads (FPKM) \pm standard error of the mean (SEM). 
Table 4. Expression of selected genes involved with prostaglandins synthesis or signaling in endometrium and conceptuses

\begin{tabular}{|c|c|c|c|c|c|c|c|}
\hline Gene name & Gene description & \multicolumn{3}{|c|}{ HF vs SF Pregnant Endometrium } & \multicolumn{3}{|c|}{ HF vs SF Conceptus } \\
\hline PG synthase & & $\mathrm{FPKM}^{1} \mathrm{HF}(\mathrm{P})$ & $\mathrm{FPKM}^{1} \mathrm{SF}(\mathrm{P})$ & FDR & FPKM $^{1} \mathrm{HF}$ & $\mathrm{FPKM}^{1} \mathrm{SF}$ & FDR \\
\hline PTGS2 & Prostaglandin-synthase 2 & $33 \pm 4$ & $40 \pm 5$ & 0.86 & $1071 \pm 111$ & $666 \pm 95$ & 0.13 \\
\hline PTGES & Prostaglandin E synthase & $2 \pm 0.5$ & $2 \pm 0.2$ & 1.00 & $14 \pm 2$ & $18 \pm 3$ & 0.35 \\
\hline PTGFS & Prostaglandin F synthase & $7 \pm 6$ & $2 \pm 1$ & 0.19 & $0.2 \pm 0.1$ & $0.5 \pm 0.3$ & 0.43 \\
\hline PTGIS & Prostaglandin I 2 synthase & $3 \pm 0.4$ & $4 \pm 0.2$ & 0.86 & $16 \pm 1$ & $12 \pm 2$ & 0.19 \\
\hline Receptors & & \multicolumn{3}{|c|}{ Open vs Preg Endometrium } & \multicolumn{3}{|c|}{ HF vs SF Pregnant Endometrium } \\
\hline Endometrium & & FPKM $^{1}$ Open & FPKM $^{1}$ Preg & FDR & $\mathrm{FPKM}^{1} \mathrm{HF}(\mathrm{P})$ & $\mathrm{FPKM}^{1} \mathrm{SF}(\mathrm{P})$ & FDR \\
\hline PTGER2 & Prostaglandin E Receptor 2 & $5 \pm 0.3$ & $5 \pm 0.4$ & 0.29 & $6 \pm 0.6$ & $5 \pm 0.4$ & 0.90 \\
\hline PTGER4 & Prostaglandin E Receptor 4 & $3 \pm 0.2$ & $3 \pm 0.2$ & 0.06 & $2 \pm 0.2$ & $3 \pm 0.3$ & 0.59 \\
\hline PPARA & Peroxisome proliferator-activated receptor alpha & $17 \pm 1$ & $15 \pm 1$ & 0.30 & $15 \pm 1$ & $15 \pm 1$ & 1.00 \\
\hline PPARD & Peroxisome proliferator-activated receptor delta & $8 \pm 0.2$ & $8 \pm 0.4$ & 0.49 & $8 \pm 0.5$ & $8 \pm 0.7$ & 0.92 \\
\hline Receptors & & \multicolumn{3}{|c|}{ Long vs Short Conceptus } & \multicolumn{3}{|c|}{ HF vs SF Conceptus } \\
\hline Conceptus & & FPKM $^{1}$ Long & FPKM $^{1}$ Short & FDR & FPKM $^{1} \mathrm{HF}$ & $\mathrm{FPKM}^{1} \mathrm{SF}$ & FDR \\
\hline PTGFR & Prostaglandin F Receptor (Fp) & $4 \pm 1$ & $2 \pm 1$ & 0.18 & $4 \pm 0.9$ & $1 \pm 0.2$ & 0.04 \\
\hline PPARD & Peroxisome proliferator-activated receptor delta & $5 \pm 1$ & $6 \pm 1$ & 0.73 & $6 \pm 0.6$ & $4 \pm 0.4$ & 0.07 \\
\hline PPARG & Peroxisome proliferator-activated receptor gamma & $3 \pm 0.3$ & $2 \pm 0.3$ & 0.48 & $3 \pm 0.2$ & $2 \pm 0.3$ & 0.10 \\
\hline Endometrium & & \multicolumn{3}{|c|}{ Preg vs Open Endometrium } & \multicolumn{3}{|c|}{ HF vs SF Pregnant Endometrium } \\
\hline PG transporter & & FPKM $^{1}$ Open & FPKM $^{1}$ Preg & FDR & $\mathrm{FPKM}^{1} \mathrm{HF}(\mathrm{P})$ & $\mathrm{FPKM}^{1} \mathrm{SF}(\mathrm{P})$ & FDR \\
\hline SLCO2A1 & Solute carrier organic anion transporter family member $2 \mathrm{~A} 1$ & $3 \pm 0.3$ & $3 \pm 0.3$ & 1.00 & $3 \pm 0.5$ & $3 \pm 0.1$ & 0.95 \\
\hline Conceptus & & \multicolumn{3}{|c|}{ Long vs Short Conceptus } & \multicolumn{3}{|c|}{ HF vs SF Conceptus } \\
\hline PG transporter & & FPKM $^{1}$ Long & FPKM $^{1}$ Short & FDR & FPKM $^{1} \mathrm{HF}$ & $\mathrm{FPKM}^{1} \mathrm{SF}$ & FDR \\
\hline SLCO2A1 & Solute carrier organic anion transporter family member $2 \mathrm{~A} 1$ & $7 \pm 2$ & $5 \pm 1$ & 0.63 & $7 \pm 1$ & $3 \pm 1$ & 0.02 \\
\hline
\end{tabular}

${ }^{1}$ Data is presented as fragments per kilobase of transcript per million mapped reads (FPKM) \pm standard error of the mean (SEM). 
Table 5. Fatty acid differences in the ULF of fertility-classified heifers

\begin{tabular}{ccc}
\hline Comparisons & \multicolumn{2}{c}{ Significantly different fatty acids in ULF } \\
\hline Preg vs Open & None & Higher in Open than Preg \\
HF Preg vs Open & $22: 0,20: 0,20: 1 \mathrm{n}-11$ & Higher in Preg than Open \\
SF Preg vs Open & $24: 3$ & Higher in HF than SF \\
Only Preg HF vs SF & $15: 0,24: 2,18: 1,22: 5 w 6,22: 4,22: 2,20: 3,20: 2$ & Higher in SF than HF \\
& $8: 0$ & Higher in HF than SF \\
Only Open HF vs SF vs IF & $22: 1$ & Higher in HF than SF and IF \\
\hline
\end{tabular}


Table 6. Lipids differentially abundant in the ULF of open and pregnant HF heifers

\begin{tabular}{cccc}
\hline ID & Preg/Open Fold Change (FC) & $\log$ 2(FC) & P-value \\
\hline Phosphatidylcholine (34:1) & 5.28 & 2.40 & 0.01 \\
Phosphatidylcholine (34:2) & 2.74 & 1.46 & 0.01 \\
Phosphatidylcholine (36:2) & 3.17 & 1.66 & 0.01 \\
Phosphatidylcholine (34:2) & 2.88 & 1.53 & 0.01 \\
Phosphatidylcholine (36:1) & 3.18 & 1.67 & 0.01 \\
Triacylglyceride (16:0_18:1_18:1) & 2.39 & 1.25 & 0.05 \\
Triacylglyceride (18:0_18:1_18:1) & 2.31 & 1.21 & 0.09 \\
Acylcarnitine (4:0) & 3.59 & 1.84 & 0.18 \\
Acylcarnitine (2:0) & 2.52 & 1.34 & 0.33 \\
Triacylglyceride (18:1_18:1_18:1) & 0.38 & -1.38 & 0.44 \\
\hline
\end{tabular}




\section{SECTION V:}

\section{Analysis of the Uterine Lumen from Fertility-Classified Heifers: II Interferon-tau, Proteomics and Metabolomics}

Joao G.N. Moraes, Susanta K. Behura, Jeanette V. Bishop, Thomas R. Hansen, Thomas W. Geary, Thomas E. Spencer 


\section{ABSTRACT}

The current experiment investigated pregnancy induced changes in uterine luminal fluid (ULF) of previously fertility-classified heifers with focus on interferon tau (IFNT), proteins and metabolites. IFNT concentrations in ULF were higher in pregnant heifers classified as high fertile (HF) than in subfertile (SF) animals. Although there were no differences in IFNT mRNA levels between HF and SF conceptuses, the increased IFNT abundance in ULF from HF heifers were presumably due to conceptus size. Nevertheless, no differences in endometrium expression of selected classical and nonclassical interferon-stimulated genes (ISGs) were observed, suggesting that IFNT signaling in the endometrium of pregnant $\mathrm{HF}$ and SF heifers was similar, and not a major cause of reduced uterine receptivity in SF animals. As expected, pregnancy induced significant changes in the proteomics, and several proteins found to be increased by pregnancy, were also increased in the ULF of pregnant HF than SF heifers, including proteins involved in energy metabolism (ACAA2, ENO1, TKT, PGD), oxidative stress (GSS), amino acid metabolism (PSPH, GOT1, LAP3, WARS, AHCY) and cell proliferation and differentiation (RAC1, HSPB1, CDC42). In the metabolomics, pregnancy induced 271 differential metabolites in the ULF of HF heifers, but no differences were identified in ULF of SF heifers that were pregnant or open. The majority of the metabolites that increased in the ULF of pregnant HF than SF heifers were associated with energy and amino acid metabolism. Hence, the observed differences in ULF proteome and metabolome are hypothesized to influence uterine receptivity and consequently conceptus development and survival. 


\section{INTRODUCTION}

During mammalian embryonic development, the oocyte is fertilized in the ampulla of the oviduct forming a zygote [88], which undergo several mitotic divisions to form a morula [89] that enters the uterus between days 4 to 6 post-mating in cattle [90]. The morula then develops into a blastocyst that contains an inner cell mass (ICM) and a blastocoele or central cavity surrounded by a monolayer of trophectoderm (TE) cells [91]. Upon blastocyst formation, two lineages of cells differentiate within the ICM: the epiblast (EPI) and the differentiated primitive endoderm (PrE) [456]. The cells from the ICM are pluripotent and able to form all three embryonic germ layers: the endoderm, mesoderm and ectoderm [92]. The transcription factor POU5F1 (also known as Oct-4) is a key regulator of pluripotency in mice embryos [457], and in ruminants, it has been reported to suppress the transcription of interferon tau (IFNT) by mononuclear TE cells during the blastocyst stage [458].

IFNT is the pregnancy recognition signal in ruminants, and it prevents luteolysis in cattle by suppressing expression of oxytocin receptor (OXTR) in the uterine luminal (LE) and glandular epithelia (GE), which abrogates the uterine luteolytic mechanism [108, 112, 113]. IFNT is a type 1 interferon (IFN) produced exclusively by mononuclear TE cells of the elongating conceptus in ruminants, and in cattle it is secreted predominantly between days 15-24 [109-111]. The transcriptional regulation of IFNT expression has been recently reviewed [122] and transcription factors such as POU5F1 [123] and T-box protein eomesodermin (EOMES) [124] have been reported to suppress IFNT expression, while others such as ETS2 [125-127], distal-less 3 (DLX3) [128], CDX2 [124, 129] and GATA2/3 [130, 131] have been implicated with increased IFNT transcription. Additionally, a co-activator, the cAMP-response element-binding protein-binding protein 
(CREBBP), is expressed by the elongating ovine conceptus and can stimulate IFNT transcription [132]. Uterine derived factors also regulate IFNT transcription by the conceptus. For instance, colony stimulated factor 1 (CSF1), interleukin 3 (IL3), and fibroblast growth factor-2 (FGF2) are expressed by the endometrium LE and GE in sheep, and have been shown to induce conceptus expression of IFNT in vitro [133-135].

The denominated classical signaling of interferons (IFNs) is through the JAK/STAT (janus kinase/signal transduce and activator of transcription) pathway, but IFNs also activates non-classical signaling cascades, such as PI3K and p38 MAP kinase pathways [121]. The bovine endometrium and conceptus express the type I interferon receptor (IFNAR) [114, 115], which is a heterodimeric receptor composed of IFNAR1 and IFNAR2 subunits [116]. Thus, the elongating conceptus secretes IFNT that signals through IFNAR in the endometrium and stimulates the expression of classical and non-classical interferon-stimulated genes (ISGs), that along with progesterone induced changes in endometrium, modulate uterine gene expression which is essential for establishing uterine receptivity in ruminants $[98,117,119,120]$. Although there are no detectable differences in uterine transcriptome of pregnant and cyclic heifers prior to day 14 , by day 16 , the endometrium transcriptome is greatly affected by the presence of a conceptus [253]. Pregnancy-induced changes in the endometrium transcriptome are hypothesized to influence uterine secretome composition, affecting conceptus development and pregnancy success $[94,192,459]$. For instance, pregnancy increases the availability of basic (e.g. arginine, lysine, histidine) acidic (e.g. aspartic acid/aspartate, glutamic acid/glutamate) and neutral amino acids (e.g. glutamine, isoleucine, leucine, phenylalanine, tyrosine and valine) in the uterine lumen during the preimplantation period in cattle and sheep $[85,139,460]$. Additionally, pregnancy modulates the availability of proteins in the uterine lumen during 
the preimplantation period in cattle $[81,459]$. Alterations in the profile of uterine luminal components during early pregnancy are a result of complex conceptus-endometrium interactions, which regulates conceptus and endometrium gene expression, ensures corpus luteum (CL) maintenance, provides substrates for cell proliferation and differentiation while preventing cell damage through oxidative stress, and induces uterine receptivity, to support conceptus growth and pregnancy establishment. Thus, it is possible that heifers with reduced fertility have altered uterine environment which is less adequate to support conceptus growth and survival. Hence, in order to identify changes in the uterine luminal constituents which are associated with increased or reduced uterine capacity to support pregnancy, the current experiment utilized heifers that were previously fertility-classified as high fertile (HF; 100\% pregnancy rate), subfertile (SF; 25-33\% pregnancy rate), or infertile (IF; 0\% pregnancy rate) using serial transfer ( $n=3-4$ rounds) of a single IVP embryo (Grade 1) on day 7 followed by pregnancy determination on day 28. Interestingly, conceptus development and survival by day 14 (7 days post-transfer) was not different among fertility-classified heifers and only minimal differences in their endometrium transcriptome were observed on day 14 [328]. Nonetheless, in a subsequent study, we demonstrated that on day 17 (10 days post-transfer), pregnancy rate was higher in HF (71\%) and SF (90\%) than IF (20\%) heifers. Day 17 conceptuses were longer in HF (mean $10.6 \mathrm{~cm}$; range $1.2-32.2 \mathrm{~cm}$ ) than $\mathrm{SF}$ (mean $4.7 \mathrm{~cm}$; range $1.5-13.5 \mathrm{~cm}$ ) heifers, and the endometrial and conceptus transcriptome was dysregulated in SF heifers (Figure S1A and S1B, representative figure from [179]). Because differences in endometrial transcriptome can translate into differences in the components available in the uterine lumen [192], in the present study, we assay the uterine luminal fluid (ULF) for IFNT, and conducted global proteomics and untargeted metabolomics in the ULF, to gather information related to the 
mechanisms influencing conceptus development and survival in heifers with distinct uterine capacity to support pregnancy, and to further explore the differences in uterine luminal components that are induced by pregnancy.

\section{MATERIALS AND METHODS}

Animals. All animal procedures were conducted in accordance with the Guide for the Care and Use of Agriculture Animals in Research and Teaching and approved by the Institutional Animal Care and Use Committees of the USDA-ARS Fort Keogh Livestock and Range Research Laboratory and the University of Missouri.

Collection of Uterine Luminal Fluid. Fertility-classified heifers (HF, n=22; SF, n=14; IF, $\mathrm{n}=6$ ) were synchronized to estrus (day 0 ) and received two in vivo-produced embryos on day 7. All heifers were slaughtered on day 17 (10 days post-transfer), and the uterine lumen gently flushed with $20 \mathrm{ml}$ of sterile and filtered 1X PBS. The conceptuses were removed, if present, the ULF clarified by centrifugation $\left(3000 \mathrm{xg}\right.$ at $4{ }^{\circ} \mathrm{C}$ for $\left.15 \mathrm{~min}\right)$, and the supernatant carefully removed with a pipette, mixed, divided into aliquots, frozen in liquid nitrogen, and stored at $-80^{\circ} \mathrm{C}$ until analyzed.

IFNT Analysis. Bovine IFNT was generated as a glycosylated recombinant protein (rbIFNT) using bovine trophoblast protein 1 cDNA (bTP509) as template [461] and human HEK cells at Colorado State University in collaboration with a biopharma company (J.V. Bishop and T.R. Hansen, manuscript in preparation). Purified rbIFNT was used to generate monoclonal antibody in mice $(9.1 .1 ; 16.2 \mu \mathrm{g} / \mathrm{ml})$ and polyclonal antibody in goats $(5.3$ $\mu \mathrm{g} / \mathrm{ml})$, which were used as capture and biotinylated detector antibodies, respectively, in a 
sandwich Enzyme Linked Immunosorbent Assay (ELISA). The range of detection for this ELISA was $100 \mathrm{pg}$ to $3,000 \mathrm{pg}$ and the limit of detection for this assay was $100 \mathrm{pg} / \mathrm{ml}$. This ELISA has been validated to be specific for IFNT, and does not cross-react with IFN $\omega$, IFN $\alpha / \beta$ or IFN $\gamma$. Uterine flush samples from open $(\mathrm{HF}, \mathrm{n}=6 ; \mathrm{SF}, \mathrm{n}=1 ; \mathrm{IF}, \mathrm{n}=4)$ and pregnant (HF, $\mathrm{n}=15 ; \mathrm{SF}, \mathrm{n}=9 ; \mathrm{IF}, \mathrm{n}=1$ ) heifers were analyzed in the same assay. Uterine flushing was analyzed as neat (undiluted) samples or at dilutions of 1:10 or 1:500 in order to detect IFNT in the linear range of the assay.

ULF Proteomics. ULF proteomics was conducted by the Proteomics Center of the University of Missouri, Columbia, MO. Two procedures were used to investigate the proteomic profile of ULF from pregnant ( $\mathrm{n}=5$ for per group: $\mathrm{HF}$ and $\mathrm{SF}$ ) and open ( $\mathrm{n}=5$ for per group: HF, SF and IF) fertility classified heifers. In the first procedure, ULF proteins were precipitated, and in-solution digestion performed. For a possibly increase in the number of proteins identified, a second procedure was conducted in which precipitated proteins were first loaded in a $12 \%$ acrylamide SDS-PAGE gel for size separation, and protein digestion was performed in slices of SDS-PAGE gels.

Description of the in-solution digestion method. Proteins in a total of $1 \mathrm{~mL}$ of ULF were precipitated using 4 volumes of 5\% trichloroacetic acid in 100\% of ice-cold acetone solution. Samples were vortexed and incubated at $-22^{\circ} \mathrm{C}$ for 24 hours for protein precipitation. Samples were then centrifuged at $16,000 \mathrm{~g}$ for 10 minutes, and the supernatant discarded. Protein pellets were resuspended in a solution of urea/ammonium bicarbonate (6M urea, $100 \mathrm{mM}$ ammonium bicarbonate), digested with trypsin (www.osa.sunysb.edu/Proteomics/ProteinDigestPrep), and peptides purified by large- 
format, 100uL, C18 tips (according to the manufacturer's instructions, Pierce/Thermo Scientific).

Description of the SDS-PAGE method. ULF protein concentrations were first determined using the Qubit ${ }^{\circledR}$ Protein Assay Kit with a Qubit ${ }^{\circledR}$ 3.0 Fluorometer, and variables volumes of ULF containing $200 \mu \mathrm{g}$ of protein were aliquoted. ULF proteins were precipitated as described in the in-solution method, and the protein pellet was resuspended in $20 \mathrm{uL}$ of $1 \mathrm{X}$ laemmli buffer (60mM Tris-HCl, pH 6.8, 10\% glycerol, 2\% SDS, and 100mM DTT), and loaded on one of three $12 \%$ acrylamide SDS-PAGE gels (Figure S2). After staining with colloidal coomassie blue, the gels were destained in water and each lane excised into 8 slices. Slices were arrayed in deep-well microtiter plates and frozen at $-80^{\circ} \mathrm{C}$ until processing. Samples were trypsin digested according to the available protocol (http://proteomics.missouri.edu/protocols) for digestion of coomassie-stained 1D gel bands. Peptides were then lyophilized and resuspended in $40 \mu \mathrm{L}$ solution of $5 \%$ acetonitrile and $1 \%$ formic acid. Half of the sample $(20 \mu \mathrm{L})$ was transferred to autosampler vials, and liquid chromatography tandem mass spectrometry (LC-MS/MS) performed on a LTQ Orbitrap XL ${ }^{\mathrm{TM}}$ mass spectrometer (ThermoFisher Scientific, Waltham, MA, USA).

LTQ orbitrap mass spectrometry and protein identification. A full-loop injection $(18 \mu \mathrm{L})$ of sample was loaded onto a C8 trap column (pepmap100, ThermoFisher Scientific, Waltham, MA, USA). Peptides were eluted from the trap column and separated on a $25 \mathrm{~cm}$ x $150 \mu \mathrm{m}$ inner diameter pulled-needle analytical column packed with HxSIL C18 reversed phase resin (Hamilton Co.) with a step gradient of acetonitrile at $400 \mathrm{~nL} / \mathrm{min}$. The Proxeon Easy nLC HPLC system is attached to an LTQ Orbitrap XL ${ }^{\mathrm{TM}}$ mass spectrometer. Liquid 
chromatography gradient conditions were initially $5 \% \mathrm{~B}(\mathrm{~A}: 0.1 \%$ formic acid in water, B: $99.9 \%$ acetonitrile, $0.1 \%$ formic acid), followed by 2 min ramp to $10 \%$ B. Gradient of 10 $20 \% \mathrm{~B}$ over $35 \mathrm{~min}$, gradient of $20 \% \mathrm{~B}$ to $30 \% \mathrm{~B}$ over $40 \mathrm{~min}$, gradient of $30 \% \mathrm{~B}$ to $90 \% \mathrm{~B}$ over $5 \mathrm{~min}$, hold at $90 \% \mathrm{~B}$ for $22 \mathrm{~min}$, ramp back to $(1 \mathrm{~min})$ and hold at $(5 \mathrm{~min})$ initial conditions.

Fourier Transform Mass Spectrometer (FTMS) data were collected $(30,000$ resolution, 1 microscan, 300-1800m/z, profile, AGC 5e5) and then each cycle (approximately 3 seconds) the 9-most-abundant peptides (ignore +1 ions, ignore trypsin autolysis ions, pick peptides with $>1,000$ counts) were selected for MSMS ( $2 \mathrm{~m} / \mathrm{z}$ mass window, $35 \%$ normalized collision energy, centroid). Dynamic exclusion was applied with following parameters: repeat count 1 , repeat duration 30sec, exclusion list max 500, exclusion duration 180sec. Raw data was copied to the Sorcerer2 IDA (SageN Research) and peak lists prepared using ReAdW. Bovine protein entries were retrieved from NCBI using a "protein" search with the keyword bovine. A total of 126,866 Bos taurus entries were downloaded in FASTA format. A reversed sequence decoy database was generated using DecoyDBCreator V0.1 (http://www.p3db.org/p3db1.0/tools/DecoyDBCreator/) in which forward and reversed sequences were concatenated into a single FASTA file (253,732 total sequences). Sequest searches were performed with trypsin as enzyme, 2 missed cleavages allowed, carbamidomethyl cysteine as a fixed modification, oxidized methionine as variable mod, $25 \mathrm{ppm}$ mass tolerance on precursor ions, and 1Da on fragment ions.

Scaffold (version Scaffold 4.0.6.1, Proteome Software Inc., Portland, OR) was used to validate MS/MS based peptide and protein identifications. Peptide identifications were accepted if they exceeded specific database search engine 
thresholds (XCorr $>1.5$ ) and with a mass accuracy of $<10 \mathrm{ppm}$. Protein identifications were accepted if they contained at least 2 identified peptides. The minimum number of unique peptides was set at 2 in order for a protein to be identified. Peptide threshold was set at $95 \%$ peptide probability, with +2 accepted charge, and parent mass tolerance of 10 parts per million. Using the parameters above, the decoy False Discovery Rate (FDR) was calculated to be $1.1 \%$ on the protein level and $0.0 \%$ on the spectrum level [462]. Further analysis was conducted on peptides identified as exclusive and unique to each protein following the removal of protein clusters. Total spectrum counts for proteins were used for comparisons and statistical analysis.

Untargeted Metabolomics. Global metabolomics analysis of ULF from day 17 pregnant $(\mathrm{HF}, \mathrm{n}=5 ; \mathrm{SF}, \mathrm{n}=5)$ and open (HF, $\mathrm{n}=5 ; \mathrm{SF}, \mathrm{n}=5 ; \mathrm{IF}, \mathrm{n}=5$ ) heifers was conducted by the Southeast Center for Integrated Metabolomics (SECIM) at the University of Florida, Gainesville, FL. All samples were extracted following a cellular extraction procedure without pre-normalization to the sample protein content.

Global metabolomics profiling was performed on a Thermo Q-Exactive Oribtrap mass spectrometer with Dionex UHPLC and autosampler. All samples were analyzed in positive and negative heated electrospray ionization with a mass resolution of 35,000 at $\mathrm{m} / \mathrm{z} 200$ as separate injections. Separation was achieved on an ACE 18-pfp 100 x $2.1 \mathrm{~mm}$, $2 \mu \mathrm{m}$ column with mobile phase $\mathrm{A}$ as $0.1 \%$ formic acid in water and mobile phase B as acetonitrile. This is a polar embedded stationary phase that provides comprehensive coverage, but have some limitations for the coverage of very polar species. The flow rate was $350 \mu \mathrm{L} / \mathrm{min}$ with a column temperature of $25^{\circ} \mathrm{C}$. A total of $4 \mu \mathrm{L}$ of each sample was 
injected for negative ion mode analysis and $2 \mu \mathrm{L}$ was injected for positive ions mode analysis.

\section{Statistical Analyses}

Statistical analyses for measurements of IFNT in ULF were conducted using SAS (SAS Institute Inc., Cary, NC). IFNT concentrations were assessed for normality using the UNIVARIATE procedure, and because it was determined not normally distributed, it was rank transformed. The effect of pregnancy status (pregnant vs open), conceptus number (one vs two) and fertility classification on ULF IFNT concentrations were determined by analysis of variance (ANOVA) using the GLM procedure. Post-test comparisons were

conducted using the LSMEANS statement with the Fisher's protected LSD option. Pearson's correlation for conceptus size and ULF IFNT concentrations were determined using the CORR procedure. Of note, the correlation analysis included only heifers which had only one conceptus present in the uterus, to remove the possible confounding effect of conceptus number on ULF IFNT concentrations.

Statistical analysis of the proteomics data was performed in Scaffold (version Scaffold 4.0.6.1, Proteome Software Inc., Portland, OR). Significant proteins were identified based on Fisher's exact test for comparisons including two treatments (e.g. Pregnant vs Open), and ANOVA with Fisher's LSD post-hoc adjustment when the analyses contained more than two treatments (e.g. HF vs SF vs IF). Enrichment analysis (http://www.geneontology.org) was performed to identify pathways that were overrepresented among significantly different proteins identified in ULF.

Statistical analysis of the metabolomics data was performed using the web server C 4.0 (http://www.metaboanalyst.ca) [408-410, 463, 464]. For this analysis, a table matrix 
of $\mathrm{m} / \mathrm{z}$ peak intensities with samples in columns and features in rows were created and imported to MetaboAnalyst 4.0. Data from positive and negative ion modes were separately subjected to statistical analyses. Data filtering was performed based on the interquartile range to identify and remove low-quality data points, and then, the data was normalized by the sum method, log transformed and scaled using the auto scaling method. A t-test was used to investigate if features were differently expressed for comparisons including two treatments (e.g. Pregnant vs Open), and ANOVA with Fisher's LSD posthoc adjustment was used when the analyses contained more than two treatments (e.g. HF vs SF vs IF). Fold change (FC) analysis was conducted to detect whether the abundance of differential metabolites increased or decreased in ULF for each comparison. The calculation of FC is based on the ratio between two group means (e.g. pregnant/open), and the standard fold change threshold of 2 was set for all analyses. Deferential metabolites were further investigated using the metabolite set enrichment analysis (MSEA) module of MetaboAnalyst 4.0, to identify biologically meaningful patterns associated with the significantly altered metabolites. To account for multiple comparisons, a false discovery rate $(\mathrm{FDR}<0.05)$ was applied for all analyses. Additionally, using the human database of MetaboAnalyst 4.0, joint pathway analyses that combined the uterine transcriptome data from fertility-classified heifers [179] with the differential metabolites detected in the ULF were conducted, in order to further explore biological pathways that were overrepresented among fertility-classified heifers during early pregnancy.

\section{Integration of uterine luminal content and endometrium and conceptus} transcriptome. To further investigate the biology of subfertility, we integrated the present 
ULF data with transcriptome data from endometrium and conceptuses which have been performed in the same group of animals and recently published [179].

To explore the relationship of endometrium transcriptome and ULF composition, we first compared data from day 17 endometrium from pregnant $(\mathrm{HF}, \mathrm{n}=5 ; \mathrm{SF}, \mathrm{n}=5)$ and open ( $\mathrm{HF}, \mathrm{n}=5 ; \mathrm{SF}, \mathrm{n}=5$ ) heifers, to detect differences in endometrium gene expression which are induced by pregnancy. Then, we compared the endometrium transcriptome of only pregnant heifers $(\mathrm{HF}, \mathrm{n}=5$ versus $\mathrm{SF}, \mathrm{n}=5)$, to explore differences in endometrium gene expression that could be associated with the detected differences in ULF composition, and related to the pregnancy loss occurring in SF heifers.

A similar approach was used to investigate the association of ULF composition and conceptus transcriptome. Because there is natural variation in conceptus length among conceptuses collected in the same day during the period of conceptus elongation in cattle $[29,36,94,328,330]$ and the conceptus transcriptome changes as it develops [94], we first analyzed day 17 conceptus transcriptome data from $\operatorname{HF}(n=15)$ and SF heifers $(n=7)$ that were either short $(\mathrm{n}=11$; mean length: $2.5 \pm 0.4 \mathrm{~cm})$ or long $(\mathrm{n}=11$; mean length: $14.5 \pm$ $1.9 \mathrm{~cm}$ ) to explore differences in the transcriptome of conceptuses that were likely due to stage of development. Then, transcriptome of HF $(n=17)$ and SF $(n=10)$ conceptuses were compared for the same set of genes of interest, to investigate the relationship of ULF composition on conceptus transcriptome, in order to explore the mechanisms associated to the retarded growth of SF conceptuses and reduced pregnancy success in SF heifers.

\section{RESULTS}

IFNT in the ULF. As expected, IFNT was not detected in the ULF of open heifers (Figure 1A). Among pregnant heifers, conceptus number did not influence $(P=0.22)$ ULF IFNT 
concentrations (Figure 1B). Consistent with differences in conceptus length (Figure S1B), IFNT concentrations in ULF was higher in pregnant HF than SF $(\mathrm{P}=0.045)$ and IF $(\mathrm{P}=$ 0.02) heifer(s) (Figure 1C). In addition, the amount of IFNT in the ULF was correlated ( $\mathrm{r}$ $=0.78, \mathrm{P}<0.01)$ with conceptus length (Figure 1D).

Integrating ULF IFNT and transcriptome data. There were no differences in abundance of IFNT transcripts in short compared to long conceptuses, or between HF and SF conceptuses (Table 1). In addition, endometrium expression of IFNT receptors (IFNAR1 and IFNAR2) was not affected by pregnancy and not different among pregnant $\mathrm{HF}$ and SF heifers. Expression of oxytocin receptor (OXTR) which was decreased in the endometrium by pregnancy, did not differ among pregnant HF and SF heifers. Furthermore, the expression of selected classical and nonclassical ISGs in the endometrium which increased by pregnancy were not different comparing only pregnant HF vs SF endometrium (Table 1).

The expression of selected genes involved with the transcriptional control of IFNT by the conceptus and endometrium [122] are presented in Table S1. Although the expression of POU5F1 by conceptuses was not significantly different between short and long $(\mathrm{FDR}=0.20)$ or between $\mathrm{HF}$ and $\mathrm{SF}$ conceptuses $(\mathrm{FDR}=0.11)$, a trend for higher expression of POU5F1 in less developed conceptuses was observed, as POU5F1 expression was increased by 2.1 folds in short than long conceptuses, and by 2.8 folds in SF than HF conceptuses. Additionally, EOMES expression by day 17 conceptuses was very low $(\mathrm{FPKM}<1)$. Interestingly, conceptus from HF heifers had higher expression of IFNT transactivators DLX3 and GATA3 compared to SF conceptuses, and GATA3 and CREBBP also tended $($ FDR $<0.1)$ to be increased in HF than SF conceptuses. 
Furthermore, among uterine secreted factors that stimulates IFNT transcription, colony stimulating factor 1 (CSF1) was increased in pregnant compared to open endometrium, but no differences were observed in the endometrium of pregnant $\mathrm{HF}$ and SF heifers (Table S1). Additionally, endometrium expression of FGF2 on day 17 was not affected by pregnancy, or between pregnant HF and SF heifers, and endometrium expression of CSF2 and IL3 was very low (FPKM $<1)$.

ULF Proteomics. There was a total of 699 proteins identified using the in-solution method (Dataset S1) and 899 proteins identified using the SDS-PAGE method (Dataset S2). As expected, the SDS-PAGE method had higher sensitivity, and therefore a greater number of proteins were identified by this procedure.

The abundance of 167 (Dataset S3) and 446 (Dataset S4) proteins were differently present in the ULF of pregnant compared to open heifers for the in-solution and SDSPAGE methods, respectively, and 103 of those proteins were differently abundant in both procedures (Table 2; Dataset S5).

In regard to the 103 different proteins in both methods, the abundance of 62 proteins increased and 41 decreased in pregnant compared to open ULF (Dataset S5). Interestingly, pathways associated with the 62 proteins that increased in pregnant ULF using the Reactome database included amino acid biosynthesis and metabolism (CKMT1, ACADVL, ACAA1, ENO1, ALDH2, ACAT1, GOT2, P4HB, GOT1, ALDOC, AHCY, PGD, CAT, ACO2, IDH2, FABP3, ALDOA, PSPH, FH, ATIC, PSAT1, PGM2, ABHD14B, HSD17B10 and DDAH2), metabolism of carbohydrates (ENO1, GOT1, GOT2, ALDOC, PGD, ALDOA, PGM2) and TCA cycle (ACO2, IDH2, FH) (Table S2). Conversely, pathways associated with the 41 proteins that increased in open compared to 
pregnant ULF using the Reactome database included intraflagellar transport due to the increased abundance of 3 cytoskeletal proteins (TUBB2B, TUBA1A, TUBB5), and regulation of insulin secretion, due to the increased abundance of 3 G-protein subunits (GNAI2, GNB2, GNAQ) in open ULF (Table S3).

Among all comparisons performed on the proteomics data (Table 2), 51\% of the significant proteins identified using the in-solution method were also significant in the SDS-PAGE method. Thus, because the SDS-PAGE method had higher sensitivity, the description of the subsequent results was focused on the data generated by the SDS-PAGE procedure.

The abundance of selected top 10 most significant proteins that increased in pregnant than open ULF (Table 3) included embryonic secreted factors (TKDP1, PAG11), nuclear-envelop proteins (Lamin A/C; LMNA), mitochondrial proteins (DLD, ACAA1, ACAA2, HSPD1, HSPA9, GLUD1), and glutathione synthase (GSS). Likewise, the selected top 10 most significant proteins that increased in open than pregnant ULF (Table 3) included cytoskeletal proteins (EZR, 2P4N, MYO1B), G-protein subunits (GNAQ, GNAI2) and other membrane-bound proteins (PAS-6/7, FAM234A, RARRES1, GPC1), and a protein of the coagulation system (Factor V).

Furthermore, there were 221 proteins differently present in the ULF of pregnant HF and SF heifers, in which the abundance of 142 proteins increased and 79 decreased in pregnant HF than SF ULF (Dataset S6, Figure 2). Panther pathways associated with the 142 proteins that increased in pregnant HF than SF ULF included vitamin B6 metabolism, amino acid metabolism (asparagine and aspartate biosynthesis, serine glycine biosynthesis), energy metabolism (pyruvate metabolism, pentose phosphate pathway, ATP synthesis and glycolysis), p38 MAPK pathway, and cytoskeletal regulation by Rho GTPase 
(Table S4, Figure 2). Panther pathways associated with the 79 proteins that increased in pregnant SF ULF compared to HF were related to hemostasis and included the plasminogen activating cascade and blood coagulation pathways (Table S5, Figure 2).

There was a total of 48 differently abundant proteins in the ULF of open fertilityclassified heifers (Dataset S7, Figure 3) but there were no pathways associated with the differently abundant proteins.

\section{Integrating ULF proteomics with transcriptome data from endometrium and} conceptuses. Among the 62 proteins commonly increased by pregnancy in both methods (Dataset S5), 56 were expressed by the endometrium based on RNA-seq data [179]. The remaining 6 proteins not expressed by the endometrium includes conceptus secreted factors (PAG11, a precursor for trophoblast Kunitz domain protein (TKDP1; GenInfo Identifier: 296481028) and TKDP1), a precursor of serpin A3-7-like protein (LOC784932; GenInfo Identifier: 985701132) which was neither expressed by the endometrium or conceptuses, and two proteins not well annotated (GenInfo Identifier: 89611 and 2323392). The proteins determined to be expressed exclusively by the conceptus were PAG11, TKDP1 and the TKDP1 precursor. Additionally, there were 11 proteins (ACO2, GDA, HSPA9, PREP, EEF2, HSD17B10, MTAP, PGM2, PSAT1 and TPI1) which increased in the ULF by pregnancy that were determined to be encoded only by genes expressed by the endometrium, not by conceptuses.

Additionally, the expression of $45 \%$ (27/60) of the genes encoding proteins commonly increased in the ULF by pregnancy were found to be increased in the endometrium of pregnant than open heifers (Figure 4A, Dataset S8), indicating that these proteins are upregulated in the endometrium by pregnancy. Interestingly, the abundance of 
$56 \%(15 / 27)$ of these proteins were also increased in pregnant HF than SF ULF (LAP3, PSPH, ENO1, WARS, QPRT, ATIC, MDH2, AHCY, ACAA2, CAP1, GOT1, HSPA9, ACTN4, LOC615277 and GSS), but none of them were increased in pregnant SF than HF ULF (Dataset S6). Conversely, the expression of only 13\% (5/39; 2 proteins not well annotated GenInfo Identifier: 7547266, 7547965) of the genes encoding proteins that increased in open ULF were found to be increased in the endometrium of open heifers (Figure 4B, Dataset S9), suggesting that these proteins are downregulated in the endometrium by pregnancy. Of those, the abundance of FCGBP was decreased, but TUBB and TUBA1A increased in pregnant HF than SF ULF.

Metabolomics. There was a total of 1,378 features detected (122 identified and 1,256 unknown) in the positive ion mode (Dataset S10) and 551 features detected (84 identified and 467 unknown) in the negative mode (Dataset S11). Identified metabolites included amino acids and amino acid derivatives, ions, carbohydrates, purines, polyphenols, lipids, and other constituents. A summary of the differential metabolites (FDR $<0.05)$ detected in the ULF according to fertility classification and pregnancy status is presented on Table 4 .

Overall, there were 315 differential metabolites (70 identified) in the ULF of pregnant versus open heifers (Datasets S12 and S13). A striking difference was observed in pregnancy induced changes in the metabolites found in the uterine lumen of $\mathrm{HF}$ and SF heifers. In HF heifers, there were 271 differential metabolites (67 identified) comparing ULF from pregnant and open heifers (Datasets S14 and S15), but surprisingly, no significant differences in metabolite composition were observed in the ULF of SF heifers that were pregnant or open (Table 4). 
Additionally, there were 13 differential metabolites in the ULF of pregnant HF and SF heifers, but only one (L-Methionine) identified (Datasets S16 and S17), and its concentration increased in pregnant HF than SF ULF. Furthermore, in the comparison among only open fertility-classified heifers, there was 1 unidentified metabolite $(\mathrm{m} / \mathrm{z}$ 109, retention time: $8.5 \mathrm{~min}$ ) which was increased in the ULF of SF than HF and IF heifers (Dataset S18).

Results of FC analysis (FC > 2) comparing pregnant and open ULF are presented on Dataset S19 and S20, and Figures S3A and S3B. The top 20 metabolites with highest differences FC are summarized in Table 5. Of note, the abundance of 76\% (145/192) of the metabolites with FC $>2$ were increased in pregnant compared to open ULF.

Results of FC analysis comparing only ULF from pregnant HF and SF heifers are presented on Datasets S21 and S22, and the top 20 identified metabolites with highest differences in FC are summarized in Table 6. The abundance of 59\% (102/173) of the metabolites with FC $>2$ were increased in HF compared to SF ULF, further indicating that pregnancy induced changes in uterine luminal metabolites were greater in $\mathrm{HF}$ than SF heifers.

Metabolite set enrichment analysis (MSEA) identified significant pathways associated with the differential metabolites between pregnant and open ULF (Figure 5A), and the top 5 pathways were urea cycle, glycine and serine metabolism, glutamate metabolism, and arginine and proline metabolism. Furthermore, the top 5 most significant pathways associated the differential metabolites between pregnant or open HF heifers included urea cycle, ammonia recycling, glycine and serine metabolism, arginine and proline metabolism, and the Warburg effect (Figure 5B). 
Additionally, joint pathways analysis identified several pathways which were overrepresented among genes (FDR $<0.05)$ and metabolites $(\mathrm{FDR}<0.05)$ that increased by pregnancy. These pathways included key biological events during early pregnancy, including biosynthesis and metabolism of amino acids, lipids, and carbohydrates (Dataset S23 Figure 6A). Interestingly, pathways associated with differently expressed genes (FDR $<0.05)$ and metabolites $(\mathrm{FC}>2)$ between pregnant $\mathrm{HF}$ than SF endometrium and ULF, respectively, included amino acid biosynthesis and metabolism (e.g. phenylalanine, tyrosine, tryptophan, glutamine and arginine), energy metabolism (e.g. TCA cycle, pentose phosphate pathway), and lipid metabolism (e.g. metabolism of glycerophospholipids and steroid biosynthesis) (Dataset S24 and Figure 6B).

\section{Expression of genes encoding amino acid transporters by the endometrium and}

conceptuses. Because the majority of the differential metabolites were associated with amino acid metabolism, we further investigated the expression of amino acid transporters by the endometrium and conceptuses.

Endometrium expression of 5 genes encoding amino acid transporters was increased (SLC15A3, SLC7A1, SLC15A1, SLC7A9, and SLC3A2) and of 3 decreased (SLC7A6, SLC7A3 and SLC6A14) by pregnancy (Dataset S25). Additionally, among pregnant heifers, the expression of SLC7A1, a transporter of cationic amino acids (arginine, lysine and ornithine) [465], was upregulated in HF than SF endometrium (Dataset S26).

Conceptus expression of amino acid transporters did not differ among short and long conceptuses (Dataset S27), but the expression of SLC7A6 was increased in HF conceptuses, and SLC6A19 was increased in SF conceptuses (Dataset S28). The top 5 most 
expressed amino acid transporters in day 17 conceptuses were SLC3A2, SLC15A4, SLC43A2, SLC15A1 and SLC7A8.

\section{DISCUSSION}

In our recent work, endometrial responses to pregnancy in fertility-classified heifers were assessed by comparing the endometrium transcriptome of nonpregnant with pregnant animals. This analysis found 3,422 differently expressed genes (DEGs) in the endometrium of HF heifers, but pregnancy resulted in only 1,095 DEGs in the endometrium of SF heifers (Figure S1A). This diminished endometrial response to pregnancy was hypothesized as the main cause of reduced uterine receptivity in SF heifers [179]. The overall differences observed in the current study further supports the theory of dysregulated conceptus-endometrium interactions in SF heifers resulting in compromised conceptus development and failure to establish pregnancy. For instance, there were 271 deferential metabolites in the ULF of HF heifers that were pregnant or open, but no significant changes in ULF metabolite profile was observed among SF heifers that were pregnant or not. Several metabolites associated with glutamine metabolism were increased in pregnant HF than SF ULF, such as 3-hydroxy-3-methylglutarate by 6.6 folds, N-methylL-glutamate by 4.6 folds, and glutarate by 3.5 folds. Although glutamine is a nonessential amino acid that can be synthesized from glucose, it is a key substrate for highly proliferative cells, and some cancer cells lines have been described as 'glutamine addicted' because they cannot survive without exogenous glutamine $[159,160]$. Glutamine is an important donor of nitrogen for synthesis of nonessential amino acids (that are used for synthesis of new proteins) and nucleotides, and glutamine synergizes with essential amino acids to activate the mechanistic target of rapamycin (mTOR) complex 1 (mTORC1) [466]. 
Activation of mTORC1 promotes cell growth and proliferation, and mediate the expression of several genes that regulates cell metabolism [467], playing important role during conceptus elongation [166].

Cancer cells and preimplantation embryos metabolize glucose preferably through aerobic glycolysis rather than oxidative phosphorylation, which is a metabolic adaptation of rapidly proliferative cells known as Warburg effect $[140,154,157]$. One of the most notable characteristics of cells undergoing the Warburg effect is that these cells uptake more glucose and produce more lactic acid than noncancer cells $[156,157]$. This is a result of pyruvate being diverged from the TCA cycle and metabolized to form lactic acid [140], and glutamine metabolism provides a carbon source for the synthesis of lactic acid [158]. Although less ATP is generated through the aerobic process $(2 \mathrm{~mol}$ of ATP per mol of glucose compared to $38 \mathrm{~mol}$ of ATP for oxidative phosphorylation), this alternative glucose metabolism diverts glucose to create macromolecular precursors for synthesis of fatty acids, amino acids, and carbohydrates [140]. In fact, the pentose phosphate pathway (PPP) plays a key role during in this process, producing ribose sugars for nucleotide synthesis, and generating sufficient levels of nicotinamide adenine dinucleotide phosphate (NADPH) for the biosynthesis of macromolecules [468]. Interestingly, in the present study, the abundance of two key enzymes which participates in the PPP were upregulated in the ULF of pregnant HF than SF heifers; transketolase (TKT) was increased by 47 folds, and 6phosphogluconate dehydrogenase (PGD) was increased by 2 folds in the ULF of pregnant HF than SF heifers. Additionally, the Warburg effect was one of the most significant pathways associated with the differential metabolites identified between pregnant and open ULF, and with the metabolites uniquely increased in HF ULF by pregnancy. Thus, taken together, these results demonstrate increased availability of substrates required for 
conceptus development in pregnant HF than SF uterine lumen, which likely contributed to the increased rate of development of HF conceptus, and consequently, with the higher pregnancy rate of HF heifers.

Histidine is an essential amino acid, and thus it cannot be synthesized de novo and must be provided in the diet [469]. Histidine availability in the uterine lumen during the preimplantation period has been observed to increase in cattle [85, 460] and sheep [139]. In the present experiment, histidine increased by 3.5 folds in pregnant than open ULF, and the expression of the histidine transporter (SLC15A3) increased by 4.6 folds in the endometrium by pregnancy. The SLC15A3 transporter is an ISG [460], and although IFNT concentrations were higher in ULF of pregnant HF than SF, SLC15A3 expression by the endometrium did not differ among pregnant fertility classified heifers.

Glutamate and glutamine are precursors of arginine [470]. Arginine is a precursor of nitric oxide (NO) and polyamines (putrescine, spermidine, spermine and agmatine) which regulates key events during early pregnancy, such as angiogenesis, placentation and embryonic development $[166,471,472]$. The secreted phosphoprotein 1 (SPP1; also known as osteopontin), an extracellular matrix protein which is secreted by the uterine LE $[77,473]$, interacts with arginine and plays important role on conceptus elongation and attachment through the activation of mTOR complexes 1 and 2 (MTORC1 and MTORC2) $[166,474]$. Interestingly, in the present study, the expression of a cationic amino acid (arginine, lysine and ornithine) transporter SLC7A1 [465] was increased in the endometrium by pregnancy, and also increased in the endometrium of pregnant HF than SF heifers on day 17. Furthermore, the concentrations of arginine and lysine in the ULF were significantly increased (FDR $<0.01$ ) by pregnancy, and $\mathrm{N}$-alpha-acetyl-L-Lysine, an amino acid derived from lysine, was increased by 3.4 folds in the ULF of pregnant HF than 
SF heifers. Lysine is another essential amino acid, and have been observed to induce cell proliferation in vitro and in vivo [475]. Lysine can be converted into carnitine [476], and two amino acids derivative of carnitine were upregulated in pregnant HF than SF ULF; the abundance of isovalerylcarnitine increased by 7.3 folds, and acyl-carnitine (5-OH) by 2.5 folds. The primary role of carnitine is associated with the transport of long-chain fatty acids from the cytosol into the mitochondria for $\beta$-oxidation, as acyl CoA (long chain fatty acid bound to coenzyme A) is not permeable to the inner mitochondrial membrane [477, 478]. The increase in acyl-carnitine, composed by carnitine bound to acyl CoA, may indicate increased abundance of long-chain fatty acid in the ULF of pregnant HF than SF heifers. Additionally, L-carnitine also possess antioxidant [479] and anti-inflammatory [480] properties, which are important biological processes during early pregnancy [481, 482].

The importance of arginine and glutamine in porcine embryo development have been demonstrated [483-485]. Additionally, the knockdown of SLC7A1 in the ovine trophectoderm using morpholino antisense oligonucleotide (MAO) resulted in retarder conceptus development [470], indicating the importance of cationic amino acids during conceptus growth in ruminants. In the present experiment, the expression of SLC7A6, which mediates mediates arginine efflux in exchange with glutamine [486, 487] was increased in HF than SF conceptuses, and perhaps suggests abnormal arginine and in glutamine metabolism in SF conceptuses.

L-Methionine was increased by 13 fold in the ULF of pregnant than open heifers, and by 18 folds in the ULF of pregnant HF than SF heifers. Methionine is the initiating amino acid for the synthesis of proteins in eukaryotes [488, 489]. Preimplantation bovine embryos are able uptake methionine from its environment [490], and the absence of methionine in the culture media reduced blastocyst rate in bovine embryos produced in 
vitro [491]. Methionine is also important for controlling oxidative stress, as it can be metabolized into cysteine, that along with glutamate and glycine are precursors of glutathione (GSH) [492, 493], a major antioxidant in mammalian cells [161]. Furthermore, GSH is also a key regulator of cell proliferation [494], and therefore might play important role during the exponential growth of the ruminant conceptus during elongation. Moreover, concentrations of GSH in the ULF have been observed to increase during early pregnancy in ewes [139]. In the present study, glutathione synthetase (GSS) was increased by 19 folds in the ULF of pregnant than open heifers, and by 3 folds in pregnant HF than SF ULF. Additionally, glutathione disulfide (GSSG), the oxidized form of glutathione (GSH), was increased by 5 folds in the ULF of open than pregnant heifers. The increase in GSSG in open ULF may be because nonpregnant heifers lacks conceptus derived glutathione reductase (GSR) and disulfide isomerases (PDI), which can converted GSSG back into GSH [161]. Under oxidative stress, glutathione peroxidases (GPx) and peroxiredoxin 6 (Prdx6) can catalyze the oxidation of GSH by hydrogen peroxide into GSSG plus water, but GSR and PDI can convert GSSG back into GSH [161].

The overall findings from the proteomics further supports our theory of dysregulated endometrium response to pregnancy in SF heifers. While the pathways associated with the proteins that increased in pregnant HF than SF heifers were involved in important biological processes during early pregnancy (e.g. energy metabolism, amino acid biosynthesis, cell proliferation and differentiation), pathways associated with proteins that increased in pregnant SF than HF ULF were associated with hemostasis (plasminogen activating cascade and blood coagulation). These results strongly suggests that HF heifer have improved uterine environment to support conceptus growth and development than SF heifers. 
The present experiment also identified 27 genes and proteins that were similarly increased by pregnancy in the endometrium and uterine lumen, respectively, and therefore the expression of those genes by the endometrium is regulated by conceptus signaling. Interestingly, $56 \%$ of these proteins were also increased in pregnant HF than SF ULF, and are involved in crucial biological events during the preimplantation period, such as energy metabolism (ACAA2, ENO1, MDH2, LOC615277, QPRT) [204, 495-498], glutathione synthesis (GSS) [499], attachment between trophectoderm and uterine LE (HSPA9) [500], de novo purine biosynthesis (ATIC) [501], amino acid metabolism (PSPH, GOT1, LAP3, WARS, AHCY) [502-506], and cytoskeletal organization and cell signaling (ACTN4, CAP1) $[507,508]$.

Regarding the IFNT results, HF heifers had higher IFNT in the ULF than SF heifers, even though no differences in IFNT gene expression were observed between HF and SF conceptuses, or between short versus long conceptuses. Longer conceptuses have greater number of trophectoderm cells, and therefore are able to secret greater amounts of IFNT. As expected, IFNT in the uterine lumen was highly correlated with conceptus size $(\mathrm{r}=0.78)$. Likewise, an earlier study reported no differences in IFNT mRNA level between bovine conceptuses that were either long $(>10 \mathrm{~cm})$ or short $(<5 \mathrm{~cm})$, but IFNT protein was substantially increased in the uterine flush from long conceptuses [109].

We further explored conceptus expression of genes that regulates IFNT transcription. The expression of IFNT transactivators DLX3, GATA3, GATA2, and CREBBP were found to increase in HF than SF conceptuses. Besides stimulating IFNT expression, this transcription factors/co-activator also mediate important biological processes during preimplantation development. For instance, DLX3 and GATA2/3 play important role mediating gene expression in the placenta, regulating trophoblast 
differentiation, angiogenesis, and coordinating embryonic-extraembryonic signaling cross-talk [509-512], and CREBBP signaling controls vital cellular processes such as cell proliferation, differentiation and apoptosis [513-515].

Among uterine secreted factors that stimulates IFNT transcription, expression of CSF1 was increased in the endometrium of pregnant than open heifers, but no differences were observed between pregnant HF and SF heifers. Additionally, IFNT signaling in the endometrium appears to be similar between pregnant HF and SF heifers, as no differences in the endometrium transcriptome were observed for the expression of genes encoding the oxytocin receptor (OXTR) or selected classical and non-classical ISGs. This result was not surprising as very low concentrations of IFNT have been shown to induce ISGs expression in vitro $[516,517]$, and a lot more is secreted during conceptus elongation [518]. Thus, it is reasonable to speculate that IFNT actions in the endometrium to establish uterine receptivity $[108,117]$ were not substantially different between pregnant HF and SF heifers. Nonetheless, the adequate conceptus size or concentrations of IFNT that are necessary to successfully induce the state of uterine receptivity in cattle are still unknown, and may vary among individual animals.

Studies in sheep and cattle supports the idea that IFNT exits the uterus and induce the expression of ISGs in maternal tissues, such as on white blood cells (WBC), CL and liver $[29,519,520]$. Interestingly, IFNT infusions into the uterine or jugular vein in sheep during days 10-13 of the estrus cycle have been shown to protect the CL against luteolysis induced on day 11 by exogenous administration of PGF2 $\alpha$, which demonstrates the luteotropic effects of IFNT [517]. Prostaglandin E2 is also luteotropic [418, 419], and PGE2 was similarly increased in the ULF of pregnant HF than SF heifers. Thus, although the CL was not investigated in the current study, it is reasonable to speculate that because 
SF heifers had shorter conceptuses [179], the concentrations of IFNT and PGE2 in the ULF were insufficient to promote adequate luteotropic effects on the CL, which consequently, contributed to the observed reduced reproductive efficiency in SF heifers.

In summary, the current study found substantial differences in uterine luminal components of fertility-classified heifers. These differences are hypothesized to occurs as a result of dysregulated conceptus-endometrium interactions in SF heifers, affecting conceptus growth and signaling, and consequently, leading to a lower pregnancy rates in SF animals.

\section{ACKNOWLEDGEMENTS}

Authors would like to thank Dr. Brian Mooney and the Charles W Gehrke Proteomics Center of the University of Missouri for the support with the proteomics, Dr. William R. Lamberson for his help with the statistical analysis, Kenneth Ladyman for his help caring for the animals, and Rick Disselhorst for coordinating the animal slaughter. This work was supported by NIH Grant 1 R01 HD072898 from the Eunice Kennedy Shriver National Institute of Child Health and Human Development. 


\section{FIGURES}

A

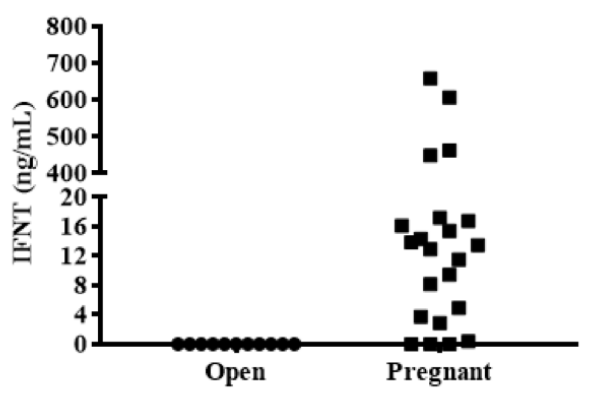

C

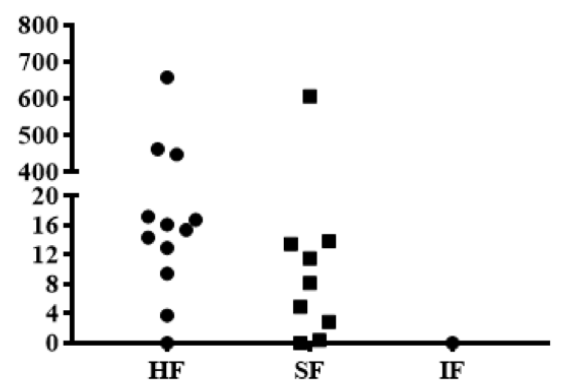

B

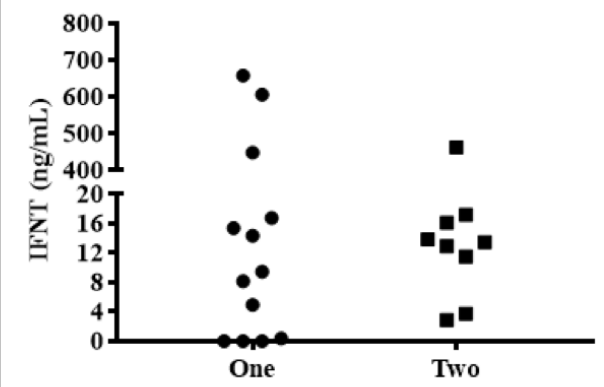

D

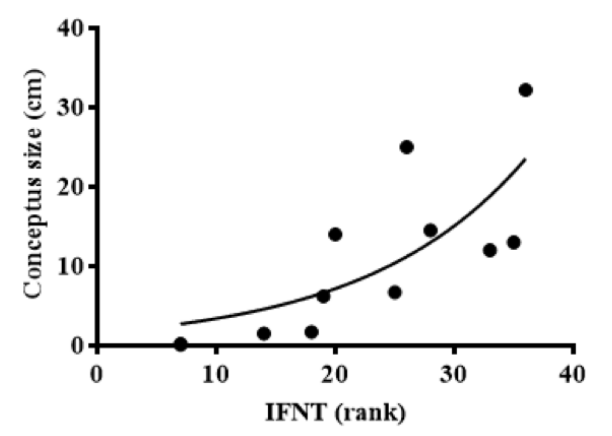

Figure 1. Concentrations of interferon tau in the ULF of fertility-classified heifers.

Interferon tau (IFNT) concentrations in the uterine luminal fluid (ULF) according to pregnancy status (A), among pregnant heifers with one or two conceptuses present in the uterine lumen (B), among pregnant fertility-classified heifers (C), and Pearson's correlation between IFNT in the ULF and conceptus size $(r=0.78, \mathrm{P}<0.01)$ (D). 


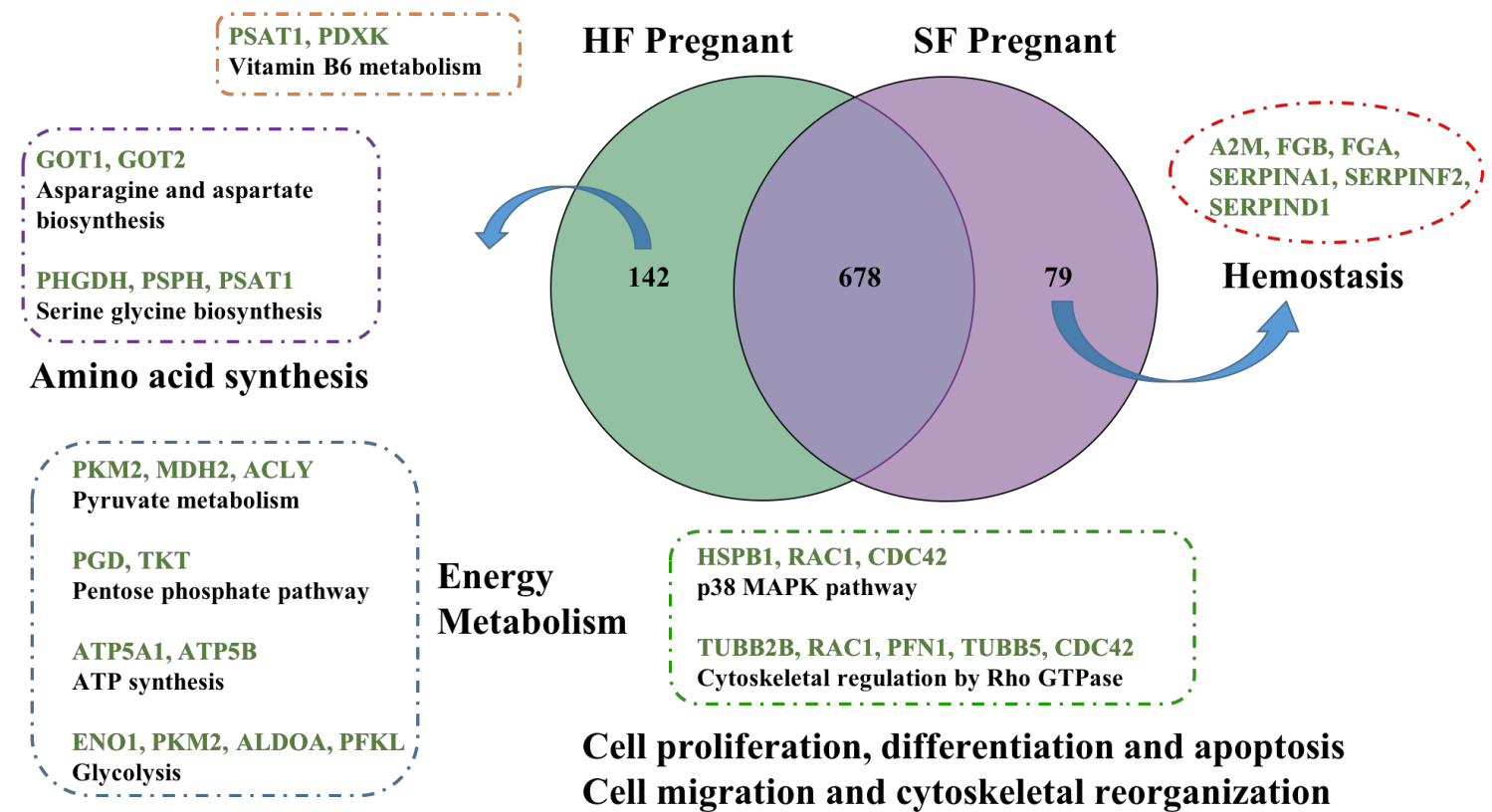

Figure 2. Venn diagram for proteins differently abundant in the ULF of pregnant HF and SF heifers according to the SDS-PAGE procedure.

Panther pathways associated with the differently abundant proteins are highlighted. 


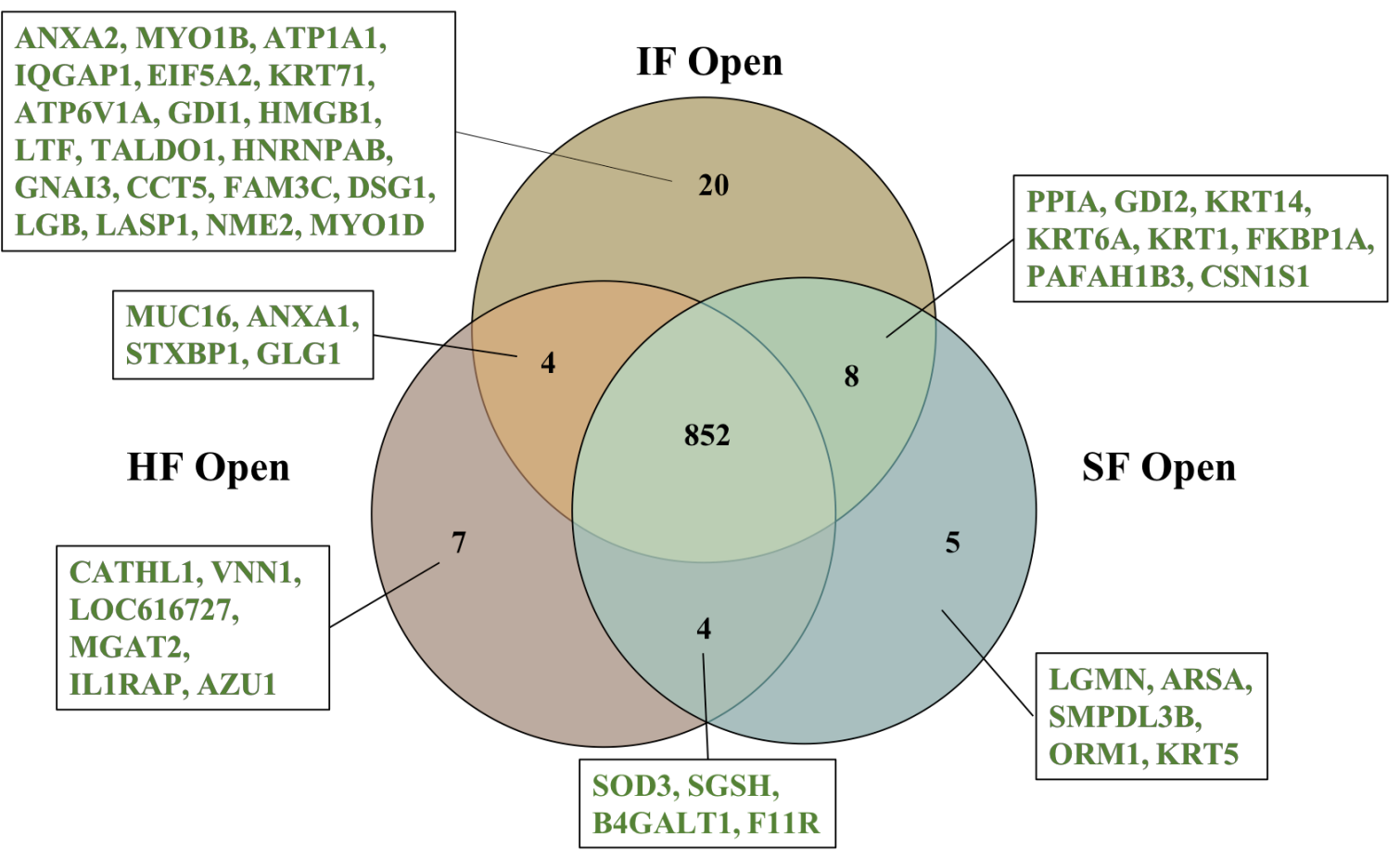

Figure 3. Venn diagram showing the differently abundant proteins among open fertility-classified heifers. 


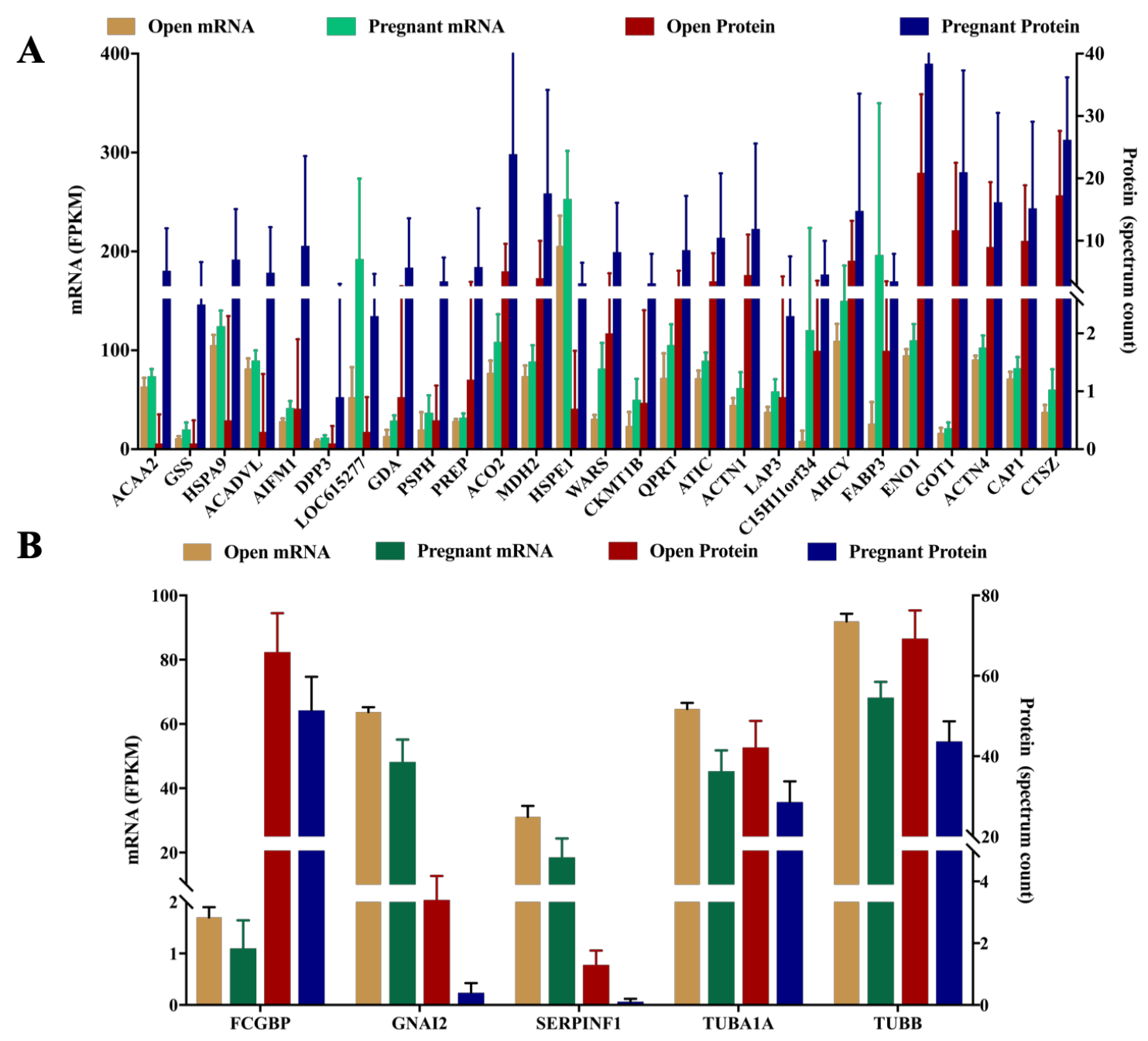

Figure 4. Selected genes/proteins hypothesized to be regulated by pregnancy based on combined data from endometrium transcriptome and ULF proteome.

Summary of genes and proteins that were similarly increased by pregnancy in the endometrium and in the ULF, respectively (A). Summary of genes and proteins that were similarly decreased by pregnancy in the endometrium and in the ULF, respectively (B). 
A

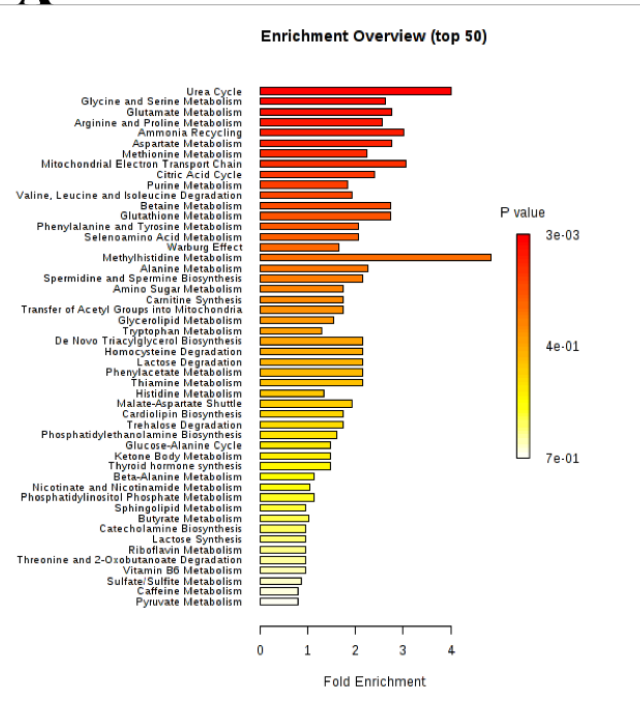

B

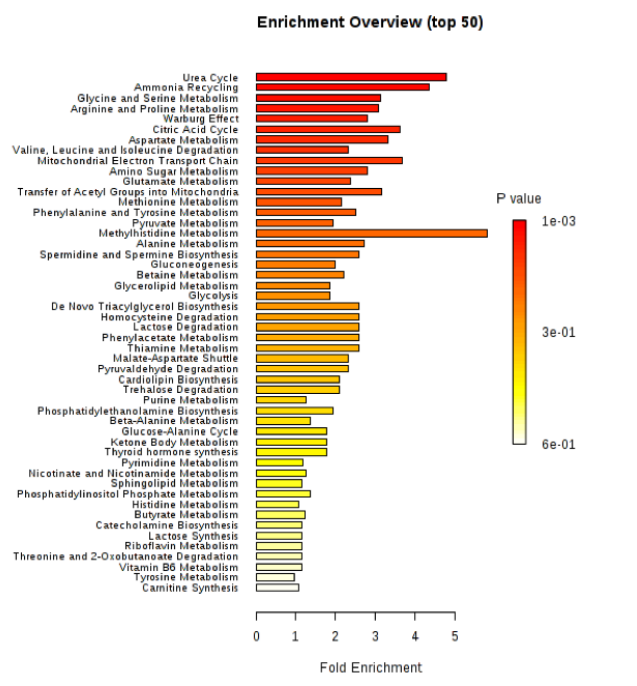

Figure 5. Metabolite set enrichment analysis (MSEA).

Pathways associated with the differential metabolites between pregnant and open ULF (A), and pathways associated with the metabolites that increased (fold change $>2$ ) in the ULF of pregnant HF than SF heifers (B). 
A

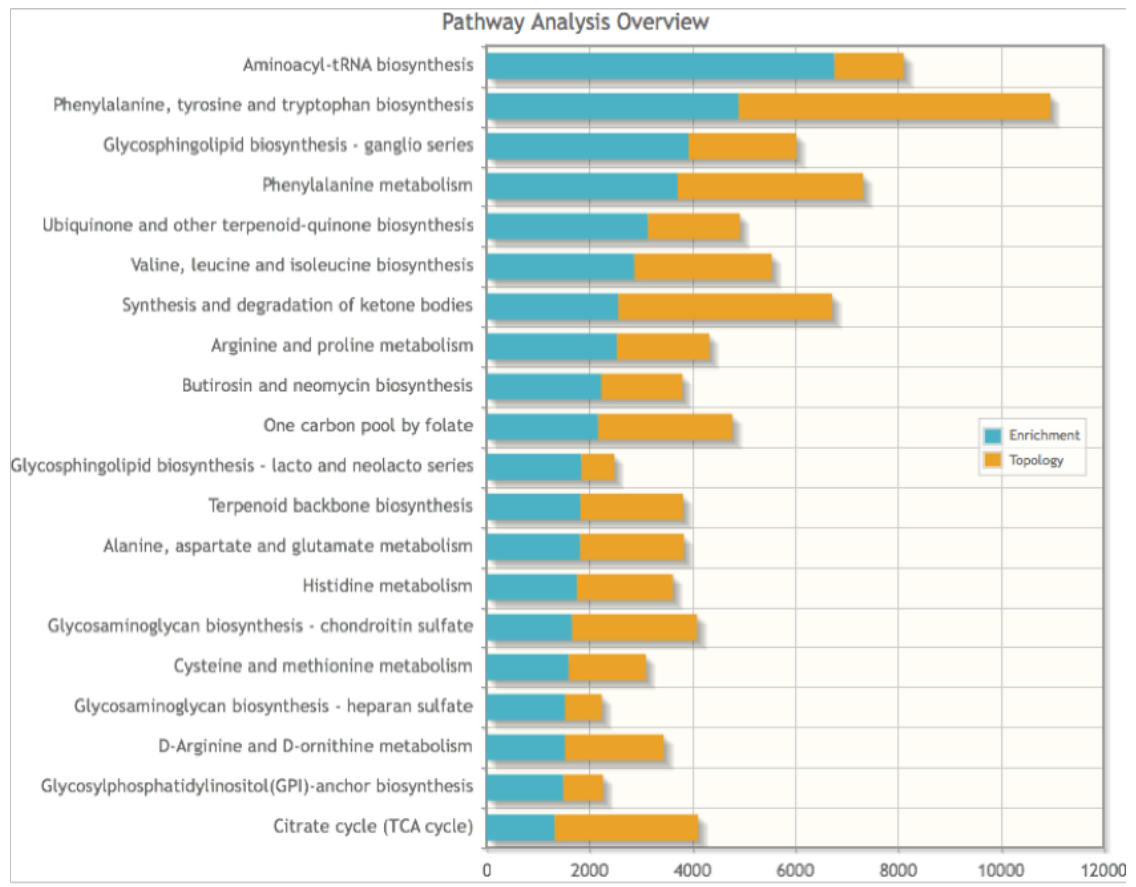

B

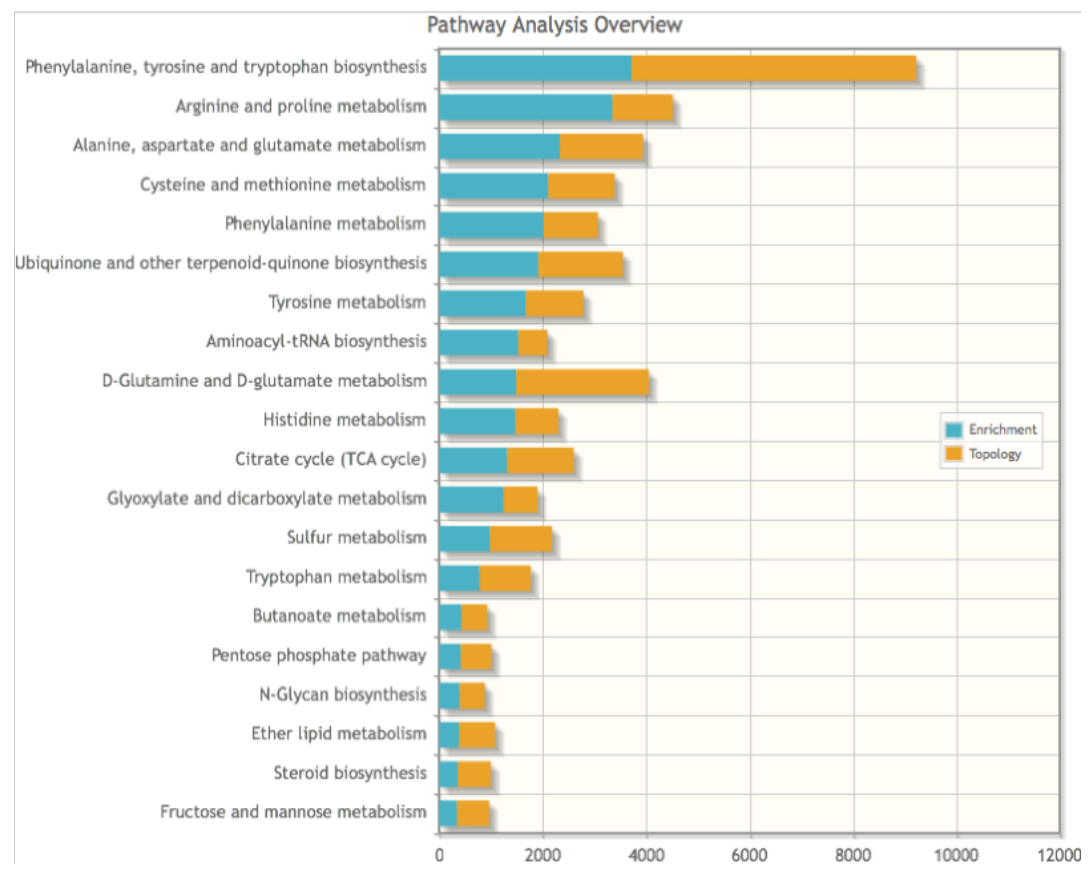

Figure 6. Joint pathway analysis for endometrium transcriptome and ULF metabolome.

Joint pathway analysis using differently expressed genes (DEG) in the endometrium and the differential metabolites detected in the ULF of open and pregnant heifers (A). Joint 
pathway analysis for the DEG in the endometrium of pregnant HF and SF heifers, with the metabolites that were differently abundant in the ULF of pregnant HF and SF heifers (B). The histograms present a summary of the joint evidence from enrichment and topological analysis. 


\section{TABLES}

Table 1. Expression of interferon tau (IFNT) by conceptuses and of selected classical and nonclassical interferon stimulated genes by the endometrium

\begin{tabular}{|c|c|c|c|c|c|c|}
\hline Conceptus & $\begin{array}{c}\text { Short } \\
\text { FPKM }^{1} \\
\end{array}$ & $\begin{array}{c}\text { Long } \\
\text { FPKM }^{1} \\
\end{array}$ & FDR & HF FPKM $^{1}$ & SF FPKM $^{1}$ & FDR \\
\hline IFNT & $3 \pm 0.9$ & $2 \pm 0.4$ & 0.98 & $2 \pm 0.4$ & $4 \pm 0.8$ & 0.21 \\
\hline Endometrium & $\begin{array}{c}\text { Open } \\
\text { FPKM }^{1} \\
\end{array}$ & $\begin{array}{c}\text { Preg } \\
\text { FPKM }^{1} \\
\end{array}$ & FDR & $\begin{array}{l}\text { HF Preg } \\
\text { FPKM }^{1}\end{array}$ & $\begin{array}{l}\text { SF Preg } \\
\text { FPKM }^{1} \\
\end{array}$ & FDR \\
\hline IFNAR1 & $24 \pm 1$ & $22 \pm 1$ & 1.00 & $22 \pm 2$ & $22 \pm 1$ & 1.00 \\
\hline IFNAR2 & $13 \pm 1$ & $14 \pm 0.5$ & 0.45 & $13 \pm 1$ & $15 \pm 0.3$ & 0.80 \\
\hline OXTR & $5 \pm 2$ & $1 \pm 0.1$ & $9.96 \mathrm{E}-05$ & $1 \pm 0.2$ & $1 \pm 0.1$ & 0.93 \\
\hline \multicolumn{7}{|l|}{ Classical ISGs } \\
\hline STAT1 & $50 \pm 2$ & $252 \pm 18$ & $6.61 \mathrm{E}-85$ & $269 \pm 30$ & $236 \pm 22$ & 0.67 \\
\hline STAT2 & $16 \pm 0.3$ & $48 \pm 4$ & $8.10 \mathrm{E}-40$ & $52 \pm 6$ & $44 \pm 6$ & 0.35 \\
\hline IRF9 & $11 \pm 1$ & $81 \pm 6$ & $2.06 \mathrm{E}-102$ & $88 \pm 8$ & $74 \pm 7$ & 0.39 \\
\hline ISG15 & $20 \pm 10$ & $1433 \pm 185$ & $6.22 \mathrm{E}-113$ & $1618 \pm 253$ & $1248 \pm 269$ & 0.30 \\
\hline $\mathrm{B} 2 \mathrm{M}$ & $721 \pm 25$ & $1956 \pm 174$ & $3.84 \mathrm{E}-31$ & $2106 \pm 209$ & $1807 \pm 285$ & 0.40 \\
\hline MIC1 & $11 \pm 2$ & $26 \pm 3$ & $1.50 \mathrm{E}-05$ & $26 \pm 4$ & $25 \pm 6$ & 0.90 \\
\hline OAS1Y & $33 \pm 6$ & $576 \pm 38$ & $1.37 \mathrm{E}-106$ & $599 \pm 51$ & $552 \pm 61$ & 0.74 \\
\hline RSAD2 & $11 \pm 4$ & $593 \pm 88$ & $2.27 \mathrm{E}-67$ & $663 \pm 116$ & $523 \pm 136$ & 0.52 \\
\hline IFIH1 & $11 \pm 1$ & $96 \pm 9$ & $1.63 \mathrm{E}-87$ & $101 \pm 10$ & $90 \pm 15$ & 0.67 \\
\hline Mx1 & $50 \pm 11$ & $934 \pm 70$ & $7.40 \mathrm{E}-116$ & $1007 \pm 111$ & $862 \pm 83$ & 0.56 \\
\hline $\mathrm{Mx} 2$ & $4 \pm 2$ & $317 \pm 42$ & $9.20 \mathrm{E}-79$ & $361 \pm 60$ & $272 \pm 57$ & 0.34 \\
\hline \multicolumn{7}{|l|}{$\begin{array}{l}\text { Non-classical } \\
\text { ISGs }\end{array}$} \\
\hline CTSL & $2 \pm 0.4$ & $4 \pm 1$ & $2.43 \mathrm{E}-02$ & $5 \pm 1$ & $3 \pm 1$ & 0.54 \\
\hline CST6 & $\begin{array}{c}196 \pm 21 \\
2558 \pm\end{array}$ & $361 \pm 50$ & 4.18E-04 & $408 \pm 95$ & $313 \pm 35$ & 0.93 \\
\hline GRP & 518 & $2889 \pm 171$ & $2.98 \mathrm{E}-02$ & $2994 \pm 204$ & $2785 \pm 292$ & 0.86 \\
\hline IGFBP1 & $79 \pm 21$ & $160 \pm 27$ & $5.71 \mathrm{E}-02$ & $144 \pm 43$ & $177 \pm 36$ & 1.00 \\
\hline SLC2A1 & $68 \pm 11$ & $153 \pm 19$ & $1.10 \mathrm{E}-05$ & $174 \pm 35$ & $132 \pm 13$ & 1.00 \\
\hline SLC5A11 & $0.7 \pm 0.1$ & $1 \pm 0.1$ & $2.07 \mathrm{E}-02$ & $1 \pm 0.3$ & $1 \pm 0.1$ & 0.94 \\
\hline
\end{tabular}

${ }^{1}$ Data is presented as fragments per kilobase of transcript per million mapped reads (FPKM) \pm standard error of the mean (SEM). 
Table 2. Proteins differentially abundant in ULF by method.

\begin{tabular}{cccc}
\hline $\begin{array}{c}\text { Compariso } \\
\text { ns }\end{array}$ & \multicolumn{2}{c}{ Significant } & \\
& $\begin{array}{c}\text { In-solution } \\
\text { method }^{\mathrm{a}}\end{array}$ & $\begin{array}{c}\text { SDS-PAGE } \\
\text { method }^{\mathrm{b}}\end{array}$ & $\begin{array}{c}\text { Common different } \\
\text { proteins }^{-}\end{array}$ \\
\hline Preg vs Open & 167 & 446 & 103 \\
HF Preg vs Open & 97 & 341 & 50 \\
SF Preg vs Open & 95 & 212 & 48 \\
Only Preg HF vs SF & 37 & 221 & 14 \\
Only Open HF, SF, & 27 & 48 & 2 \\
IF & & &
\end{tabular}

${ }^{a} A$ total of 699 proteins were identified in the in-solution procedure

${ }^{\mathrm{b}} \mathrm{A}$ Total of 899 proteins were identified in the in-solution procedure. 
Table 3. Top 10 proteins that increased or decreased in pregnant compared to open ULF

\begin{tabular}{|c|c|c|c|c|c|}
\hline Identified Proteins & GenInfo Identifier & Protein & P-value & Open ${ }^{1}$ & Preg $^{1}$ \\
\hline \multicolumn{6}{|l|}{ Proteins increased in pregnant ULF } \\
\hline Pregnancy-associated glycoprotein 11 & 28603724 & PAG11 & $<0.00010$ & 0.0 & 5.3 \\
\hline Trophoblast Kunitz domain protein 1 precursor & 296481028 & TKDP1 & $<0.00010$ & 0.0 & 4.7 \\
\hline Mitochondrial acetyl-Coenzyme A acyltransferase 1 & 156254808 & ACAA1 & $<0.00010$ & 0.0 & 4.0 \\
\hline Dihydrolipoamide dehydrogenase & 296488519 & DLD & $<0.00010$ & 0.0 & 1.1 \\
\hline 3-ketoacyl-CoA thiolase, mitochondrial & 121956694 & ACAA2 & $<0.00010$ & 0.1 & 5.2 \\
\hline Glutathione synthetase & 296481143 & GSS & $<0.00010$ & 0.1 & 2.5 \\
\hline Bovine Glutamate Dehydrogenase & 298508694 & GLUD1 & $<0.00010$ & 0.6 & 10.3 \\
\hline Heat shock $70 \mathrm{kDa}$ protein 9 & 122144079 & HSPA9 & $<0.00010$ & 0.5 & 7.0 \\
\hline Lamin $\mathrm{A} / \mathrm{C}$ & 296489648 & LMNA & $<0.00010$ & 0.1 & 2.0 \\
\hline Heat shock protein $60 \mathrm{kDa}$, mitochondrial isoform $\mathrm{X} 1$ & 982914181 & HSPD1 & $<0.00010$ & 3.0 & 28.1 \\
\hline \multicolumn{6}{|l|}{ Proteins increased in open ULF } \\
\hline PAS-6 and PAS-7 proteins & 1632779 & PAS-6/7 & $<0.00010$ & 4.5 & 0.1 \\
\hline Guanine nucleotide-binding protein $\mathrm{G}(\mathrm{q})$ subunit alpha & 158508558 & GNAQ & $<0.00010$ & 2.6 & 0.3 \\
\hline Guanine nucleotide-binding protein G(i) subunit alpha-2 & 198282135 & GNAI2 & $<0.00010$ & 3.4 & 0.4 \\
\hline Factor V & 163038 & FACTORV & $<0.00010$ & 13.9 & 2.2 \\
\hline Retinoic acid receptor responder (tazarotene induced) 1 & 112362395 & RARRES1 & $<0.00010$ & 3.7 & 0.6 \\
\hline FAM234A; Alternative name: ITFG3 & 114149324 & FAM234A & $<0.00010$ & 4.2 & 0.7 \\
\hline MYO1B protein & 151554811 & MYO1B & $<0.00010$ & 9.1 & 1.8 \\
\hline Bovine Tubulin (1jff) & 193885177 & $2 \mathrm{P} 4 \mathrm{~N}$ & $<0.00010$ & 49.1 & 10.0 \\
\hline Glypican 1 & 157279068 & GPC1 & $<0.00010$ & 3.6 & 1.0 \\
\hline Ezrin & 27806351 & EZR & $<0.00010$ & 33.3 & 14.0 \\
\hline
\end{tabular}

${ }^{1}$ Average of total spectrum count for proteins identified in the ULF of open and pregnant heifers. 
Table 4. Summary of the differential metabolites identified in the ULF.

\begin{tabular}{cccc}
\hline Comparisons & Identified Metabolites & Unknown Metabolites & Total Metabolites \\
\hline Preg vs Open & 70 & 248 & 315 \\
HF Preg vs Open & 67 & 208 & 271 \\
SF Preg vs Open & 0 & 0 & 0 \\
Preg HF vs SF & 1 & 12 & 13 \\
Open HF, SF, IF & 0 & 1 & 1 \\
\hline
\end{tabular}


Table 5. Top 20 identified metabolites with the highest differences in fold change in ULF of pregnant than to open heifers

\begin{tabular}{lcccc}
\hline Positive mode & $\mathrm{m} / \mathrm{z}$ & Retention time & Preg/Open Fold Change (FC) & log2(FC) \\
\hline L-Methionine & 150.1 & 1.4 & 13.1 & 3.7 \\
Hypoxanthine & 137.0 & 6.1 & 0.1 & -2.9 \\
Glutathione Disulfide & 307.1 & 3.9 & 0.2 & -2.4 \\
Isovalerylcarnitine & 246.2 & 8.1 & 4.7 & 2.2 \\
R-Malate & 157.0 & 1.0 & 4.4 & 2.1 \\
2-Hydroxyphenylalanine & 182.1 & 3.4 & 3.9 & 2.0 \\
L-Histidine & 156.1 & 0.7 & 3.5 & 1.8 \\
Methionine Sulfoxide & 166.1 & 0.8 & 3.5 & 1.8 \\
N-Alpha-Acetyl-L-Lysine & 189.1 & 1.2 & 3.3 & 1.7 \\
Phenylalanine & 166.1 & 6.3 & 3.2 & 1.7 \\
& & & & \\
Negative Mode & & & 29.4 & 4.9 \\
N-Methyl-L-Glutamate & 160.1 & 0.9 & 6.3 & 2.7 \\
3-Hydroxy-3-Methylglutarate & 161.0 & 2.9 & 0.2 & -2.6 \\
Inosine & 267.1 & 6.0 & 6.1 & 2.6 \\
6-Keto Prostaglandin G1 & 369.2 & 9.8 & 4.8 & 2.3 \\
N-Acetylneuraminate & 308.1 & 0.8 & 4.5 & 2.2 \\
Fumaric Acid & 115.0 & 2.0 & 4.4 & 2.1 \\
Malate & 133.0 & 1.0 & 4.3 & 2.1 \\
L-Tyrosine & 180.1 & 3.4 & 3.6 & 1.8 \\
Threonine/Homoserine & 118.1 & 0.7 & 3.5 & 1.8 \\
Glutarate & 131.0 & 4.6 & &
\end{tabular}


Table 6. Top 20 identified metabolites with the highest differences in fold change in ULF of pregnant HF than SF heifers

\begin{tabular}{|c|c|c|c|c|}
\hline Metabolites & $\mathrm{m} / \mathrm{z}$ & $\begin{array}{l}\text { Retention } \\
\text { time }\end{array}$ & $\begin{array}{l}\text { HF/SF - Fold Change } \\
(\mathrm{FC})\end{array}$ & $\begin{array}{c}\log 2(\mathrm{FC} \\
)\end{array}$ \\
\hline \multicolumn{5}{|l|}{ Increased in $\mathrm{HF}$} \\
\hline L-Methionine & 150.1 & 1.4 & 17.7 & 4.1 \\
\hline Isovalerylcarnitine & 246.2 & 8.1 & 7.3 & 2.9 \\
\hline 3-Hydroxy-3-Methylglutarate & 161.0 & 2.9 & 6.6 & 2.7 \\
\hline $\mathrm{N}$-Acetylneuraminate & 308.1 & 0.8 & 6.2 & 2.6 \\
\hline N-Methyl-L-Glutamate & 160.1 & 0.9 & 4.6 & 2.2 \\
\hline Glutarate & 131.0 & 4.6 & 3.5 & 1.8 \\
\hline N-Alpha-Acetyl-L-Lysine & 189.1 & 1.2 & 3.4 & 1.8 \\
\hline Fumaric Acid & 115.0 & 2.0 & 2.6 & 1.4 \\
\hline 4-Imidazoleacetic Acid & 127.0 & 1.0 & 2.5 & 1.3 \\
\hline Acyl-Carnitine (5-OH) & 262.2 & 6.7 & 2.5 & 1.3 \\
\hline Proprionylcarnitine & 218.1 & 6.8 & 2.4 & 1.3 \\
\hline 2-Hydroxyglutarate & 147.0 & 1.5 & 2.3 & 1.2 \\
\hline Malate & 133.0 & 1.0 & 2.2 & 1.2 \\
\hline L-Glutamic Acid & 146.0 & 0.8 & 2.1 & 1.1 \\
\hline Citrulline & 174.1 & 0.8 & 2.1 & 1.1 \\
\hline $\begin{array}{l}\text { Isobutyrylcarnitine } \\
\text { Nepsilon,Nepsilon,Nepsilon- }\end{array}$ & 232.2 & 7.4 & 2.1 & 1.1 \\
\hline Trimethyllysine & 189.2 & 0.7 & 2.0 & 1.0 \\
\hline N-Acetyl-L-Alanine & 154.0 & 2.2 & 2.0 & 1.0 \\
\hline \multicolumn{5}{|l|}{ Increased SF } \\
\hline P-Cresol Sulfate & 187.0 & 8.4 & 0.4 & -1.4 \\
\hline Adenosine 5'-Monophosphate & 348.1 & 1.5 & 0.3 & -1.9 \\
\hline
\end{tabular}


Table S1. Expression of selected genes involved with the transcriptional control of IFNT expression

\begin{tabular}{|c|c|c|c|c|c|c|}
\hline \multicolumn{7}{|l|}{ Conceptus } \\
\hline IFNT supressors & $\begin{array}{c}\text { Short } \\
\text { FPKM }^{1}\end{array}$ & Long FPKM ${ }^{1}$ & FDR & SF FPKM $^{1}$ & HF FPKM $^{1}$ & FDR \\
\hline POU5F1 & $5 \pm 2$ & $3 \pm 1$ & 0.20 & $6 \pm 2$ & $2 \pm 0.5$ & 0.11 \\
\hline EOMES & $0.3 \pm 0.2$ & $0.1 \pm 0.0$ & 0.45 & $0.3 \pm 0.2$ & $0.1 \pm 0.0$ & 0.37 \\
\hline \multicolumn{7}{|l|}{$\begin{array}{l}\text { IFNT } \\
\text { transactivators }\end{array}$} \\
\hline ETS2 & $23 \pm 3$ & $17 \pm 0.9$ & 0.54 & $28 \pm 2$ & $28 \pm 2$ & 0.89 \\
\hline CREBBP & $5 \pm 0.8$ & $4 \pm 0.3$ & 0.85 & $6 \pm 0.5$ & $9 \pm 0.5$ & 0.07 \\
\hline DLX3 & $3 \pm 0.6$ & $4 \pm 0.5$ & 0.67 & $3 \pm 0.2$ & $7 \pm 0.5$ & $7.87 \mathrm{E}-03$ \\
\hline CDX2 & $26 \pm 2$ & $22 \pm 2$ & 0.52 & $42 \pm 2$ & $35 \pm 2$ & 0.24 \\
\hline GATA2 & $27 \pm 5$ & $28 \pm 3$ & 0.58 & $37 \pm 3$ & $54 \pm 3$ & 0.08 \\
\hline GATA3 & $21 \pm 5$ & $29 \pm 4$ & 0.086 & $19 \pm 1$ & $47 \pm 4$ & $2.05 \mathrm{E}-04$ \\
\hline \multicolumn{7}{|l|}{ Endometrium } \\
\hline Maternal factors & $\begin{array}{c}\text { Open } \\
\text { FPKM }^{1}\end{array}$ & $\begin{array}{l}\text { Pregnant } \\
\text { FPKM }^{1}\end{array}$ & FDR & HF Preg FPKM ${ }^{1}$ & SF Preg FPKM ${ }^{1}$ & FDR \\
\hline CSF1 & $7 \pm 0.4$ & $14 \pm 0.8$ & $1.63 \mathrm{E}-14$ & $15 \pm 0.9$ & $13 \pm 1$ & 0.43 \\
\hline CSF2 & $0.0 \pm 0.0$ & $0.1 \pm 0.0$ & 0.23 & $0.0 \pm 0.0$ & $0.1 \pm 0.1$ & 0.86 \\
\hline IL3 & $0.0 \pm 0.0$ & $0.0 \pm 0.0$ & 0.67 & $0.0 \pm 0.0$ & $0.0 \pm 0.0$ & 1.00 \\
\hline FGF2 & $13 \pm 0.6$ & $11 \pm 0.7$ & 0.77 & $10 \pm 0.7$ & $12 \pm 1$ & 0.77 \\
\hline
\end{tabular}




\section{CONCLUSIONS AND FUTURE DIRECTIONS}

Collectively, these studies support the central hypothesis that the uterus directly influences conceptus development, and that the experimental model used to identify heifers with superior and inferior uterine capacity to support pregnancy was proven efficient. The studies conducted on day 14 established that: (1) circulating progesterone concentrations are not different among fertility-classified heifers; (2) conceptus development and survival by day 14 is not affected by fertility classification; (3) only minimal differences in endometrium transcriptome are detected among pregnant fertility-classified heifers. These results indicated that the mechanisms underlying subfertility and infertility manifests between days 14 and 28, which encompasses the period of maternal recognition of pregnancy, implantation, and beginning of placentation.

Next, uterine influences on conceptus development were investigated on day 17. Taken together, the available data indicated that the mechanism of pregnancy loss in fertility-classified heifers was manifested around the time of maternal recognition of pregnancy (Day 17), and the cause of pregnancy loss in SF heifers is likely a result of reduced endometrial response to pregnancy. Indeed, only minor differences in the endometrium transcriptome were observed among nonpregnant fertility-classified heifers on day 17 , but the response of the endometrium to pregnancy was much reduced in SF (1,095 DEGs) than HF (3,422 DEGs) heifers. Moreover, analysis of ligand-receptor interactions indicated dysregulated conceptus-endometrium interactions in SF animals, reinforcing the theory of reduced endometrial response to pregnancy in SF heifers. Differences in uterine transcriptome may influence histotroph composition and uterine structure, and thus have the potential to affect preimplantation conceptus development. 
Conceptus from HF heifers were on average twice as long than SF conceptuses on day 17 , and their transcriptomes were significantly different (1,287 DEG). The substantial differences in the transcriptome of $\mathrm{HF}$ and $\mathrm{SF}$ conceptuses was hypothesized to be mediated by differences in uterine environment rather than differences in conceptus size, as only minor differences in transcriptome were detected between long and short conceptuses from HF heifers. Importantly, many of the genes that decreased in SF compared with HF conceptuses have been implicated with abnormal embryogenesis and with embryonic mortality using knockout mouse models.

Analysis of day 17 ULF revealed new and important information regarding histotroph composition during early pregnancy, as well as provided further insights on the biology of subfertility in cattle. IFNT, the major conceptus secreted factor involved with maternal recognition of pregnancy [1-3], was more abundant in the ULF of pregnant HF than SF heifers, which was likely due to conceptus size, as there were no differences in IFNT mRNA levels between HF and SF conceptuses, or in the expression of uterinederived factors that stimulates IFNT transcription. Nonetheless, no differences were observed in endometrial expression of selected classical and non-classical ISGs. Although the actions of IFNT in the endometrium to establish uterine receptivity does not appear to be substantially different between pregnant HF and SF heifers, the endocrine effects of IFNT may influence pregnancy success. IFNT and PGE2 are luteotropic [4-6], and their concentrations were increased in the ULF of pregnant HF than SF heifers. Thus, it is reasonable to speculate that concentrations of IFNT and PGE2 that exits the uterus and supports CL function were insufficient in SF heifers, which contributed to the observed reduced reproductive efficiency in these animals. 
Glucose is a major nutrient supporting embryonic development [7-10], and although its concentration increased in the uterine lumen by pregnancy in our study, ULF glucose concentrations did not differ among fertility-classified heifers. Furthermore, only minor differences in endometrium and conceptus transcriptomes were observed for genes involved with glucose metabolism. Thus, the availability of glucose in the ULF during early pregnancy does not seem to play a major role influencing subfertility and infertility in the present study.

We found that concentrations of PGs tended to increase in the ULF by pregnancy, and in ULF of pregnant HF than SF heifers. Higher concentrations of PGs in the ULF of pregnant HF heifers is likely because they had longer conceptuses. Although pathways associated with PG signaling (through classical and nuclear receptors) in the endometrium and conceptus remains poorly understood in ruminants, PGs are essential for pregnancy success in sheep, as they stimulate a number of progesterone stimulated genes in the endometrium [11], and inhibition of PTGS2 prevents conceptus elongation [12]. Importantly, PGF2 $\alpha$ receptor (PTGFR) was the highest PG receptor expressed by the day 17 conceptus, and therefore PGF2 $\alpha$ signaling may pay important role regulating conceptus development during elongation. Additionally, PPARA which regulates FA catabolism in several tissues [13], was the highest PPAR expressed by day 17 endometrium, and perhaps have critical roles modulating uterine luminal lipid content during early pregnancy.

Surprisingly, day 17 ULF fatty acid content of pregnant and open heifers did not differ, and palmitic acid (16:0) and stearic acid (18:0) accounted for around $90 \%$ of the total fatty acid detected in the ULF. However, a number of fatty acids were increased in the ULF of pregnant HF than SF heifers, which might be a result of differences in lipid metabolism between HF and SF conceptuses, as several genes involved with fatty acid (e.g. 
ELOVL5, ELOVL7, FADS1, PLA2G15, PLA2G1B) and lipid (e.g. GPAM, CHKA, CERS1, ASAH1, CYP11A1, ACAT1) metabolism were differently expressed between HF and SF conceptuses. Furthermore, acyl-carnitine, composed of carnitine bound to acyl CoA (LCFA bound to coenzyme A), was also increased in pregnant HF than SF ULF, further supporting the results of increased LCFA in the ULF of pregnant HF than SF heifers.

The metabolism of preimplantation embryos have been suggested to be similar to that of cancer cells, which metabolizes glucose primarily through aerobic glycolysis (Warburg effect) [7], and have a high glutamine demand [14, 15]. In our data, several metabolites associated with glutamine metabolism were increased in pregnant than open ULF, and also in ULF of pregnant HF than SF heifers. Two key enzymes (PGD, TKT) that participates in the PPP, an important pathway used by cells undergoing the Warburg effect, were upregulated in the ULF of pregnant HF than SF heifers. PPP plays important role generating ribose sugars for nucleotide synthesis [16], and two precursors of purine biosynthesis (hypoxanthine, inosine), were decreased in pregnant than open ULF, perhaps indicating that these precursors were being used in an accelerated rate during pregnancy to supports conceptus growth. Additionally, the Warburg effect was one of the most significant pathways associated with the differential metabolites identified between pregnant and open ULF, and with the metabolites uniquely increased in the ULF of HF heifers by pregnancy. These results support the theory that the Warburg effect is an important metabolic process during bovine conceptus elongation, and that the rate of aerobic glycolysis was diminished in SF heifers. Moreover, several amino acids (e.g. methionine, malate, phenylalanine, histidine, lysine, arginine, tyrosine, threonine/homoserine) and intermediates of the TCA cycle (fumarate, malate) that are clearly important during early pregnancy were increased in the ULF by pregnancy. 
Additionally, several of these amino acids and TCA cycle intermediates were also increased in the ULF of pregnant HF than SF heifers, supporting our theory of dysregulated endometrial response to pregnancy in SF heifers.

Our data also provides further insights on the mechanisms regulating oxidative stress during early pregnancy in cattle. Glutathione (GSH) is a major antioxidant in mammalian cells [17], and in the present study, glutathione synthetase (GSS) was increased by 19 fold in the ULF of pregnant than open heifers, and by 3 fold in pregnant HF than SF ULF. Additionally, glutathione disulfide (GSSG), the oxidized form of glutathione (GSH), was increased by 5 fold in the ULF of open than pregnant heifers. The increase in GSSG in nonpregnant ULF perhaps indicates that oxidative stress in the uterine lumen is reduced during pregnancy, an event that could be mediated by conceptus derived glutathione reductase (GSR), which is highly expressed by the bovine conceptus during elongation.

Proteomics of the ULF demonstrated that conceptus-endometrium interactions alter the profile of proteins in the uterine lumen, and a number of proteins expressed by the endometrium were hypothesized as being regulated by pregnancy, based on data from endometrium transcriptome and ULF proteomics. Additionally, differences in the ULF proteome between pregnant HF and SF heifers indicated that HF heifers have a superior uterine environment to support conceptus development, as a number of proteins involved with energy metabolism, amino acid metabolism, and cell proliferation and differentiation were upregulated in the ULF of pregnant HF than SF heifers.

Collectively, the observed differences in histotroph composition further supports the theory dysregulated conceptus-endometrium interactions in the SF heifers, as a number of proteins, metabolites, PGs, IFNT, and lipids, all with essential roles during early pregnancy, were increased in the ULF of pregnant HF than SF heifers. However, histotroph 
composition is directly influenced by conceptus-secreted factors and conceptusendometrium interactions. Thus, the observed differences in histotroph composition may simply be because HF heifers had longer conceptuses, and not necessarily the cause of reduced fertility in SF animals. Future studies should aim to understand the mechanisms regulating the diminished endometrial response to pregnancy in SF heifers. These studies may include investigating the genome and endometrial epigenome of fertility-classified heifers. It would be particularly interesting to investigate the transcriptional regulation of genes found to be altered by pregnancy in the endometrium of HF heifers but not in SF animals. Exploring endometrial expression of microRNAs might also provide useful information regarding post-transcriptional regulation of endometrial genes. Additionally, the use of transmission electron microscopy (TEM) to further investigate the phenotype of uterine cells from fertility-classified heifers could provide valuable information related to the dysregulated conceptus endometrium-interactions in SF heifers, as the physical contact between the conceptus and endometrium is thought play an important role during conceptus elongation [18-20].

Additional research opportunities to enhance our current knowledge regarding early pregnancy events in ruminants includes: (1) improve current knowledge concerning PG signaling in the conceptus and endometrium; (2) improve current knowledge concerning lipid metabolism by conceptus and endometrium during pregnancy; (3) investigate metabolic requirements of conceptus during elongation. (4) investigate the gluconeogenic capacity of uterine cells; (4) conduct loss-of-function studies to investigate placental development; (5) investigate the mechanisms regulating conceptus elongation; (6) study the process of epigenetic reprograming of the bovine conceptus during the preimplantation period. 


\section{REFERENCES}

1. Macklon NS, Geraedts JP, Fauser BC. Conception to ongoing pregnancy: the 'black box' of early pregnancy loss. Hum Reprod Update 2002; 8:333-343.

2. Wiltbank MC, Baez GM, Garcia-Guerra A, Toledo MZ, Monteiro PL, Melo LF, Ochoa JC, Santos JE, Sartori R. Pivotal periods for pregnancy loss during the first trimester of gestation in lactating dairy cows. Theriogenology 2016; 86:239-253.

3. Spencer TE, Bazer FW. Uterine and placental factors regulating conceptus growth in domestic animals. J Anim Sci 2004; 82 E-Suppl:E4-13.

4. Bazer FW, Roberts RM. Biochemical aspects of conceptus--endometrial interactions. J Exp Zool 1983; 228:373-383.

5. Pierson RA, Ginther OJ. Ultrasonography for detection of pregnancy and study of embryonic development in heifers. Theriogenology 1984; 22:225-233.

6. Humblot P. Use of pregnancy specific proteins and progesterone assays to monitor pregnancy and determine the timing, frequencies and sources of embryonic mortality in ruminants. Theriogenology $2001 ; 56: 1417-1433$.

7. Diskin MG, Sreenan JM. Fertilization and embryonic mortality rates in beef heifers after artificial insemination. J Reprod Fertil 1980; 59:463-468.

8. Diskin MG, Morris DG. Embryonic and early foetal losses in cattle and other ruminants. Reprod Domest Anim 2008; 43 Suppl 2:260-267.

9. Maurer RR, Chenault JR. Fertilization failure and embryonic mortality in parous and nonparous beef cattle. J Anim Sci 1983; 56:1186-1189.

10. Sreenan JM, Diskin MG. The Extent and Timing of Embryonic Mortality in the Cow. Embryonic Mortality in Farm Animals 1986; 34:1-11. 
11. Ahmad N, Schrick FN, Butcher RL, Inskeep EK. Effect of persistent follicles on early embryonic losses in beef cows. Biol Reprod 1995; 52:1129-1135.

12. Sartori R, Sartor-Bergfelt R, Mertens SA, Guenther JN, Parrish JJ, Wiltbank MC. Fertilization and early embryonic development in heifers and lactating cows in summer and lactating and dry cows in winter. J Dairy Sci 2002; 85:2803-2812.

13. Sartori R, Bastos MR, Wiltbank MC. Factors affecting fertilisation and early embryo quality in single- and superovulated dairy cattle. Reprod Fertil Dev 2010; 22:151-158.

14. Ribeiro ES, Gomes G, Greco LF, Cerri RLA, Vieira-Neto A, Monteiro PLJ, Jr., Lima FS, Bisinotto RS, Thatcher WW, Santos JEP. Carryover effect of postpartum inflammatory diseases on developmental biology and fertility in lactating dairy cows. J Dairy Sci 2016; 99:2201-2220.

15. Santos JE, Thatcher WW, Chebel RC, Cerri RL, Galvao KN. The effect of embryonic death rates in cattle on the efficacy of estrus synchronization programs. Anim Reprod Sci 2004; 82-83:513-535.

16. Georgadaki K, Khoury N, Spandidos DA, Zoumpourlis V. The molecular basis of fertilization (Review). Int J Mol Med 2016; 38:979-986.

17. Lucy MC. Reproductive loss in high-producing dairy cattle: where will it end? J Dairy Sci 2001; 84:1277-1293.

18. Graden AP, Olds D, Mochow CR, Mutter LR. Causes of fertilization failure in repeat breeding cattle. J Dairy Sci 1968; 51:778-781.

19. Carvalho PD, Souza AH, Sartori R, Hackbart KS, Dresch AR, Vieira LM, Baruselli PS, Guenther JN, Fricke PM, Shaver RD, Wiltbank MC. Effects of deep-horn 
AI on fertilization and embryo production in superovulated cows and heifers.

Theriogenology 2013; 80:1074-1081.

20. Wiltbank MC, Souza AH, Carvalho PD, Cunha AP, Giordano JO, Fricke PM, Baez GM, Diskin MG. Physiological and practical effects of progesterone on reproduction in dairy cattle. Animal 2014; 8 Suppl 1:70-81.

21. Rocha A, Randel RD, Broussard JR, Lim JM, Blair RM, Roussel JD, Godke RA, Hansel W. High environmental temperature and humidity decrease oocyte quality in Bos taurus but not in Bos indicus cows. Theriogenology 1998; 49:657-665.

22. Al-Katanani YM, Paula-Lopes FF, Hansen PJ. Effect of season and exposure to heat stress on oocyte competence in Holstein cows. J Dairy Sci 2002; 85:390-396.

23. Fearon JM, Wegener PT. Relationship between fertility in cattle and the number of inseminated spermatozoa. J Reprod Fertil 2000; 119:293-308.

24. Schwartz D, Macdonald PD, Heuchel V. On the relationship between the number of spermatozoa and the probability of conception. Reprod Nutr Dev 1981; 21:979-988.

25. Thundathil J, Palasz AT, Barth AD, Mapletoft RJ. The use of in vitro fertilization techniques to investigate the fertilizing ability of bovine sperm with proximal cytoplasmic droplets. Anim Reprod Sci 2001; 65:181-192.

26. Walters AH, Eyestone WE, Saacke RG, Pearson RE, Gwazdauskas FC. Bovine embryo development after IVF with spermatozoa having abnormal morphology. Theriogenology 2005; 63:1925-1937.

27. Hayakawa H, Hirai T, Takimoto A, Ideta A, Aoyagi Y. Superovulation and embryo transfer in Holstein cattle using sexed sperm. Theriogenology 2009; 71:68-73. 28. Peippo J, Vartia K, Kananen-Anttila K, Raty M, Korhonen K, Hurme T, Myllymaki H, Sairanen A, Maki-Tanila A. Embryo production from superovulated 
Holstein-Friesian dairy heifers and cows after insemination with frozen-thawed sexsorted X spermatozoa or unsorted semen. Anim Reprod Sci 2009; 111:80-92.

29. Ortega MS, Moraes JGN, Patterson DJ, Smith MF, Behura SK, Poock S, Spencer TE. Influences of sire conception rate on pregnancy establishment in dairy cattle. Biol Reprod 2018.

30. Roche JF, Bolandl MP, McGeady TA. Reproductive wastage following artificial insemination of heifers. Vet Rec 1981; 109:401-404.

31. Hansen PJ. Early Embryonic Loss Due to Heat Stress. Bovine Reproduction 2014.

32. Revah I, Butler WR. Prolonged dominance of follicles and reduced viability of bovine oocytes. J Reprod Fertil 1996; 106:39-47.

33. Rivera FA, Mendonca LG, Lopes G, Jr., Santos JE, Perez RV, Amstalden M, Correa-Calderon A, Chebel RC. Reduced progesterone concentration during growth of the first follicular wave affects embryo quality but has no effect on embryo survival post transfer in lactating dairy cows. Reproduction $2011 ; 141: 333-342$.

34. Dunne LD, Diskin MG, Sreenan JM. Embryo and foetal loss in beef heifers between day 14 of gestation and full term. Anim Reprod Sci 2000; 58:39-44.

35. Christenson RK, Echternkamp SE, Laster DB. Oestrus, LH, ovulation and fertility in beef heifers. J Reprod Fertil 1975; 43:543-546.

36. Berg DK, van Leeuwen J, Beaumont S, Berg M, Pfeffer PL. Embryo loss in cattle between Days 7 and 16 of pregnancy. Theriogenology 2010; 73:250-260.

37. Reese ST, Pereira MHC, Edwards JL, Vasconcelos JLM, Pohler KG. Pregnancy diagnosis in cattle using pregnancy associated glycoprotein concentration in circulation at day 24 of gestation. Theriogenology 2018; 106:178-185. 
38. Pohler KG, Green JA, Geary TW, Peres RFG, Pereira MHC, Vasconcelos JLM, Smith MF. Predicting embryo presence and viability. Regulation of Implantation and Establishment of Pregnancy in Mammals: Tribute to 45 Year Anniversary of Roger V. Short's "Maternal Recognition of Pregnancy" 2015.

39. Diskin MG, Waters SM, Parr MH, Kenny DA. Pregnancy losses in cattle: potential for improvement. Reprod Fertil Dev 2016; 28:83-93.

40. LeBlanc S. Assessing the association of the level of milk production with reproductive performance in dairy cattle. J Reprod Dev 2010; 56 Suppl:S1-7.

41. Butler WR, Smith RD. Interrelationships between energy balance and postpartum reproductive function in dairy cattle. J Dairy Sci 1989; 72:767-783.

42. Butler WR, Everett RW, Coppock CE. The relationships between energy balance, milk production and ovulation in postpartum Holstein cows. J Anim Sci 1981; 53:742748.

43. Staples CR, Thatcher WW, Clark JH. Relationship between ovarian activity and energy status during the early postpartum period of high producing dairy cows. J Dairy Sci 1990; 73:938-947.

44. Hammon DS, Evjen IM, Dhiman TR, Goff JP, Walters JL. Neutrophil function and energy status in Holstein cows with uterine health disorders. Vet Immunol Immunopathol 2006; 113:21-29.

45. Lacetera N, Scalia D, Franci O, Bernabucci U, Ronchi B, Nardone A. Short communication: effects of nonesterified fatty acids on lymphocyte function in dairy heifers. J Dairy Sci 2004; 87:1012-1014. 
46. Santos JE, Bisinotto RS, Ribeiro ES, Lima FS, Greco LF, Staples CR, Thatcher WW. Applying nutrition and physiology to improve reproduction in dairy cattle. Soc Reprod Fertil Suppl 2010; 67:387-403.

47. de ST-JJR, de FAPM, de Sa WF, de MFA, Viana JH, Camargo LS, Ramos AA, Folhadella IM, Polisseni J, de Freitas C, Clemente CA, de Sa Filho MF, et al. Effect of maternal heat-stress on follicular growth and oocyte competence in Bos indicus cattle. Theriogenology 2008; 69:155-166.

48. Wolfenson D, Thatcher WW, Badinga L, Savio JD, Meidan R, Lew BJ, Braw-Tal R, Berman A. Effect of heat stress on follicular development during the estrous cycle in lactating dairy cattle. Biol Reprod 1995; 52:1106-1113.

49. Payton RR, Romar R, Coy P, Saxton AM, Lawrence JL, Edwards JL. Susceptibility of bovine germinal vesicle-stage oocytes from antral follicles to direct effects of heat stress in vitro. Biol Reprod 2004; 71:1303-1308.

50. Roth Z, Hansen PJ. Involvement of apoptosis in disruption of developmental competence of bovine oocytes by heat shock during maturation. Biol Reprod 2004; $71: 1898-1906$.

51. Ferreira RM, Ayres H, Chiaratti MR, Ferraz ML, Araujo AB, Rodrigues CA, Watanabe YF, Vireque AA, Joaquim DC, Smith LC, Meirelles FV, Baruselli PS. The low fertility of repeat-breeder cows during summer heat stress is related to a low oocyte competence to develop into blastocysts. J Dairy Sci 2011; 94:2383-2392.

52. Sakatani M, Alvarez NV, Takahashi M, Hansen PJ. Consequences of physiological heat shock beginning at the zygote stage on embryonic development and expression of stress response genes in cattle. J Dairy Sci 2012; 95:3080-3091. 
53. Sakatani M, Kobayashi S, Takahashi M. Effects of heat shock on in vitro development and intracellular oxidative state of bovine preimplantation embryos. Mol Reprod Dev 2004; 67:77-82.

54. Edwards JL, Hansen PJ. Differential responses of bovine oocytes and preimplantation embryos to heat shock. Mol Reprod Dev 1997; 46:138-145.

55. Eberhardt BG, Satrapa RA, Capinzaiki CR, Trinca LA, Barros CM. Influence of the breed of bull (Bos taurus indicus vs. Bos taurus taurus) and the breed of cow (Bos taurus indicus, Bos taurus taurus and crossbred) on the resistance of bovine embryos to heat. Anim Reprod Sci 2009; 114:54-61.

56. Stewart BM, Block J, Morelli P, Navarette AE, Amstalden M, Bonilla L, Hansen PJ, Bilby TR. Efficacy of embryo transfer in lactating dairy cows during summer using fresh or vitrified embryos produced in vitro with sex-sorted semen. J Dairy Sci 2011; 94:3437-3445.

57. Wijma R, Stangaferro ML, Kamat MM, Vasudevan S, Ott TL, Giordano JO. Embryo Mortality Around the Period of Maintenance of the Corpus Luteum Causes Alterations to the Ovarian Function of Lactating Dairy Cows. Biol Reprod 2016; 95:112. 58. Wiebold JL. Embryonic mortality and the uterine environment in first-service lactating dairy cows. J Reprod Fertil 1988; 84:393-399.

59. Ryan DP, Prichard JF, Kopel E, Godke RA. Comparing early embryo mortality in dairy cows during hot and cool seasons of the year. Theriogenology 1993; 39:719-737. 60. Cerri RL, Juchem SO, Chebel RC, Rutigliano HM, Bruno RG, Galvao KN, Thatcher WW, Santos JE. Effect of fat source differing in fatty acid profile on metabolic parameters, fertilization, and embryo quality in high-producing dairy cows. J Dairy Sci 2009; 92:1520-1531. 
61. Cerri RL, Rutigliano HM, Chebel RC, Santos JE. Period of dominance of the ovulatory follicle influences embryo quality in lactating dairy cows. Reproduction 2009; 137:813-823.

62. Cerri RL, Rutigliano HM, Lima FS, Araujo DB, Santos JE. Effect of source of supplemental selenium on uterine health and embryo quality in high-producing dairy cows. Theriogenology 2009; 71:1127-1137.

63. Hackbart KS, Ferreira RM, Dietsche AA, Socha MT, Shaver RD, Wiltbank MC, Fricke PM. Effect of dietary organic zinc, manganese, copper, and cobalt supplementation on milk production, follicular growth, embryo quality, and tissue mineral concentrations in dairy cows. J Anim Sci 2010; 88:3856-3870.

64. Hafez ESE, Hafez B. Anatomy of Female Reproduction. Reproduction in Farm Animals 2016.

65. Suarez SS, Pacey AA. Sperm transport in the female reproductive tract. Hum Reprod Update 2006; 12:23-37.

66. Milvae RA, Hinckley ST, Carlson JC. Luteotropic and luteolytic mechanisms in the bovine corpus luteum. Theriogenology 1996; 45:1327-1349.

67. Bazer FW, Wu G, Johnson GA, Kim J, Song G. Uterine histotroph and conceptus development: select nutrients and secreted phosphoprotein 1 affect mechanistic target of rapamycin cell signaling in ewes. Biol Reprod 2011; 85:1094-1107.

68. Maltepe E, Fisher SJ. Placenta: the forgotten organ. Annu Rev Cell Dev Biol $2015 ; 31: 523-552$.

69. Weiss G. Endocrinology of parturition. J Clin Endocrinol Metab 2000; 85:44214425.

70. Senger PL. Pathways to Pregnancy and Parturition. 2015. 
71. Filant J, Spencer TE. Uterine glands: biological roles in conceptus implantation, uterine receptivity and decidualization. Int J Dev Biol 2014; 58:107-116.

72. Cooke PS, Spencer TE, Bartol FF, Hayashi K. Uterine glands: development, function and experimental model systems. Mol Hum Reprod 2013; 19:547-558.

73. Zhang S, Regnault TR, Barker PL, Botting KJ, McMillen IC, McMillan CM, Roberts CT, Morrison JL. Placental adaptations in growth restriction. Nutrients 2015; 7:360-389.

74. Gray CA, Bartol FF, Tarleton BJ, Wiley AA, Johnson GA, Bazer FW, Spencer TE. Developmental biology of uterine glands. Biol Reprod 2001; 65:1311-1323.

75. Spencer TE, Kelleher AM, Bartol FF. Development and Function of Uterine Glands in Domestic Animals. Annu Rev Anim Biosci 2018.

76. Spencer TE, Gray CA. Sheep uterine gland knockout (UGKO) model. Methods Mol Med 2006; 121:85-94.

77. Gray CA, Burghardt RC, Johnson GA, Bazer FW, Spencer TE. Evidence that absence of endometrial gland secretions in uterine gland knockout ewes compromises conceptus survival and elongation. Reproduction 2002; 124:289-300.

78. Filant J, Spencer TE. Endometrial glands are essential for blastocyst implantation and decidualization in the mouse uterus. Biol Reprod 2013; 88:93.

79. Kelleher AM, Burns GW, Behura S, Wu G, Spencer TE. Uterine glands impact uterine receptivity, luminal fluid homeostasis and blastocyst implantation. Sci Rep 2016; 6:38078.

80. Bazer FW, Song G, Kim J, Dunlap KA, Satterfield MC, Johnson GA, Burghardt RC, Wu G. Uterine biology in pigs and sheep. J Anim Sci Biotechnol 2012; 3:23. 
81. Forde N, McGettigan PA, Mehta JP, O'Hara L, Mamo S, Bazer FW, Spencer TE, Lonergan P. Proteomic analysis of uterine fluid during the pre-implantation period of pregnancy in cattle. Reproduction 2014; 147:575-587.

82. Ulbrich SE, Schulke K, Groebner AE, Reichenbach HD, Angioni C, Geisslinger G, Meyer HH. Quantitative characterization of prostaglandins in the uterus of early pregnant cattle. Reproduction 2009; 138:371-382.

83. Hugentobler SA, Sreenan JM, Humpherson PG, Leese HJ, Diskin MG, Morris DG. Effects of changes in the concentration of systemic progesterone on ions, amino acids and energy substrates in cattle oviduct and uterine fluid and blood. Reprod Fertil Dev 2010; 22:684-694.

84. Keller ML, Roberts AJ, Seidel GE, Jr. Characterization of insulin-like growth factor-binding proteins in the uterus and conceptus during early conceptus elongation in cattle. Biol Reprod 1998; 59:632-642.

85. Forde N, Simintiras CA, Sturmey R, Mamo S, Kelly AK, Spencer TE, Bazer FW, Lonergan P. Amino acids in the uterine luminal fluid reflects the temporal changes in transporter expression in the endometrium and conceptus during early pregnancy in cattle. PLoS One 2014; 9:e100010.

86. Wathes DC, Wooding FB. An electron microscopic study of implantation in the cow. Am J Anat 1980; 159:285-306.

87. King GJ, Atkinson BA, Robertson HA. Development of the bovine placentome from days 20 to 29 of gestation. J Reprod Fertil 1980; 59:95-100.

88. Coy P, Garcia-Vazquez FA, Visconti PE, Aviles M. Roles of the oviduct in mammalian fertilization. Reproduction 2012; 144:649-660. 
89. Clift D, Schuh M. Restarting life: fertilization and the transition from meiosis to mitosis. Nat Rev Mol Cell Biol 2013; 14:549-562.

90. Van Soom A, Boerjan ML, Bols PE, Vanroose G, Lein A, Coryn M, de Kruif A. Timing of compaction and inner cell allocation in bovine embryos produced in vivo after superovulation. Biol Reprod 1997; 57:1041-1049.

91. Artus J, Chazaud C. A close look at the mammalian blastocyst: epiblast and primitive endoderm formation. Cell Mol Life Sci 2014; 71:3327-3338.

92. Thomson JA, Itskovitz-Eldor J, Shapiro SS, Waknitz MA, Swiergiel JJ, Marshall VS, Jones JM. Embryonic stem cell lines derived from human blastocysts. Science 1998; 282:1145-1147.

93. Bó GA, Mapletoft RJ. Evaluation and classification of bovine embryos. Animal Reproduction 2013; 10:344-348.

94. Ribeiro ES, Greco LF, Bisinotto RS, Lima FS, Thatcher WW, Santos JE. Biology of Preimplantation Conceptus at the Onset of Elongation in Dairy Cows. Biol Reprod 2016; 94:97.

95. Betteridge KJ, Eaglesome MD, Randall GC, Mitchell D. Collection, description and transfer of embryos from cattle 10--16 days after oestrus. J Reprod Fertil 1980; $59: 205-216$.

96. Heyman Y, Camous S, Fevre J, Meziou W, Martal J. Maintenance of the corpus luteum after uterine transfer of trophoblastic vesicles to cyclic cows and ewes. J Reprod Fertil 1984; 70:533-540.

97. Flechon JE, Guillomot M, Charlier M, Flechon B, Martal J. Experimental studies on the elongation of the ewe blastocyst. Reprod Nutr Dev 1986; 26:1017-1024. 
98. Spencer TE, Johnson GA, Bazer FW, Burghardt RC, Palmarini M. Pregnancy recognition and conceptus implantation in domestic ruminants: roles of progesterone, interferons and endogenous retroviruses. Reprod Fertil Dev 2007; 19:65-78.

99. Guillomot M. Cellular interactions during implantation in domestic ruminants. J Reprod Fertil Suppl 1995; 49:39-51.

100. Wooding FB. Role of binucleate cells in fetomaternal cell fusion at implantation in the sheep. Am J Anat 1984; 170:233-250.

101. Wooding FB. Current topic: the synepitheliochorial placenta of ruminants: binucleate cell fusions and hormone production. Placenta 1992; 13:101-113.

102. Wooding FBP, Osborn D, Killian GJ. Trinucleate uterine epithelial cells as evidence for White-tail Deer trophoblast binucleate cell migration and as markers of placental binucleate cell dynamics in a variety of wild ruminants. Placenta 2018; 62:3442.

103. Spencer TE, Johnson GA, Burghardt RC, Bazer FW. Progesterone and placental hormone actions on the uterus: insights from domestic animals. Biol Reprod 2004; 71:210.

104. Assis Neto AC, Pereira FT, Santos TC, Ambrosio CE, Leiser R, Miglino MA. Morpho-physical recording of bovine conceptus (Bos indicus) and placenta from days 20 to 70 of pregnancy. Reprod Domest Anim 2010; 45:760-772.

105. Spencer TE. Biological roles of uterine glands in pregnancy. Semin Reprod Med $2014 ; 32: 346-357$.

106. Spencer TE. Early pregnancy: Concepts, challenges, and potential solutions. Animal Frontiers 2013; 3:48-55. 
107. McCracken JA, Custer EE, Lamsa JC. Luteolysis: a neuroendocrine-mediated event. Physiol Rev 1999; 79:263-323.

108. Hansen TR, Sinedino LDP, Spencer TE. Paracrine and endocrine actions of interferon tau (IFNT). Reproduction 2017; 154:F45-F59.

109. Robinson RS, Fray MD, Wathes DC, Lamming GE, Mann GE. In vivo expression of interferon tau mRNA by the embryonic trophoblast and uterine concentrations of interferon tau protein during early pregnancy in the cow. Mol Reprod Dev 2006; 73:470474.

110. Forde N, Lonergan P. Transcriptomic analysis of the bovine endometrium: What is required to establish uterine receptivity to implantation in cattle? J Reprod Dev 2012; 58:189-195.

111. Ealy AD, Yang QE. Control of interferon-tau expression during early pregnancy in ruminants. Am J Reprod Immunol 2009; 61:95-106.

112. Bazer FW, Thatcher WW. Chronicling the discovery of interferon tau. Reproduction 2017; 154:F11-F20.

113. Robinson RS, Mann GE, Lamming GE, Wathes DC. The effect of pregnancy on the expression of uterine oxytocin, oestrogen and progesterone receptors during early pregnancy in the cow. J Endocrinol 1999; 160:21-33.

114. Shirozu T, Iwano H, Ogiso T, Suzuki T, Balboula AZ, Bai H, Kawahara M, Kimura K, Takahashi H, Rulan B, Kim SW, Yanagawa Y, et al. Estrous cycle stagedependent manner of type I interferon-stimulated genes induction in the bovine endometrium. J Reprod Dev 2017; 63:211-220. 
115. Wang W, Liu R, Liang X, Zhao Q, Qu P, Yao K, Jiang M, Luo Y, Zhang W, Qing S. Expression of IFNAR1 and IFNAR2 in cattle placenta during early pregnancy. Reprod Domest Anim 2018; 53:385-392.

116. de Weerd NA, Samarajiwa SA, Hertzog PJ. Type I interferon receptors: biochemistry and biological functions. J Biol Chem 2007; 282:20053-20057.

117. Spencer TE, Sandra O, Wolf E. Genes involved in conceptus-endometrial interactions in ruminants: insights from reductionism and thoughts on holistic approaches. Reproduction 2008; 135:165-179.

118. Telgmann R, Bathgate RA, Jaeger S, Tillmann G, Ivell R. Transcriptional regulation of the bovine oxytocin receptor gene. Biol Reprod 2003; 68:1015-1026. 119. Spencer TE, Burghardt RC, Johnson GA, Bazer FW. Conceptus signals for establishment and maintenance of pregnancy. Anim Reprod Sci 2004; 82-83:537-550. 120. Brooks K, Burns G, Spencer TE. Conceptus elongation in ruminants: roles of progesterone, prostaglandin, interferon tau and cortisol. J Anim Sci Biotechnol 2014; $5: 53$.

121. Platanias LC. Introduction: interferon signals: what is classical and what is nonclassical? J Interferon Cytokine Res 2005; 25:732.

122. Ezashi T, Imakawa K. Transcriptional control of IFNT expression. Reproduction 2017; 154:F21-F31.

123. Gupta R, Ezashi T, Roberts RM. Squelching of ETS2 transactivation by POU5F1 silences the human chorionic gonadotropin CGA subunit gene in human choriocarcinoma and embryonic stem cells. Mol Endocrinol 2012; 26:859-872. 
124. Sakurai T, Bai H, Bai R, Sato D, Arai M, Okuda K, Ideta A, Aoyagi Y, Godkin JD, Imakawa K. Down-regulation of interferon tau gene transcription with a transcription factor, EOMES. Mol Reprod Dev 2013; 80:371-383.

125. Ezashi T, Ealy AD, Ostrowski MC, Roberts RM. Control of interferon-tau gene expression by Ets-2. Proc Natl Acad Sci U S A 1998; 95:7882-7887.

126. Das P, Ezashi T, Gupta R, Roberts RM. Combinatorial roles of protein kinase A, Ets2, and 3',5'-cyclic-adenosine monophosphate response element-binding proteinbinding protein/p300 in the transcriptional control of interferon-tau expression in a trophoblast cell line. Mol Endocrinol 2008; 22:331-343.

127. Matsuda-Minehata F, Katsumura M, Kijima S, Christenson RK, Imakawa K. Different levels of ovine interferon-tau gene expressions are regulated through the short promoter region including Ets-2 binding site. Mol Reprod Dev 2005; 72:7-15.

128. Ezashi T, Das P, Gupta R, Walker A, Roberts RM. The role of homeobox protein distal-less 3 and its interaction with ETS2 in regulating bovine interferon-tau gene expression-synergistic transcriptional activation with ETS2. Biol Reprod 2008; 79:115124.

129. Sakurai T, Bai H, Konno T, Ideta A, Aoyagi Y, Godkin JD, Imakawa K. Function of a transcription factor CDX2 beyond its trophectoderm lineage specification. Endocrinology 2010; 151:5873-5881.

130. Bai H, Sakurai T, Kim MS, Muroi Y, Ideta A, Aoyagi Y, Nakajima H, Takahashi M, Nagaoka K, Imakawa K. Involvement of GATA transcription factors in the regulation of endogenous bovine interferon-tau gene transcription. Mol Reprod Dev 2009; 76:11431152. 
131. Bai H, Sakurai T, Bai R, Godkin JD, Imakawa K. Localization of GATA2 in the nuclear and cytoplasmic regions of ovine conceptuses. Anim Sci J 2014; 85:981-985.

132. Xu N, Takahashi Y, Matsuda F, Sakai S, Christenson RK, Imakawa K.

Coactivator CBP in the regulation of conceptus IFNtau gene transcription. Mol Reprod Dev 2003; 65:23-29.

133. Imakawa K, Helmer SD, Nephew KP, Meka CS, Christenson RK. A novel role for GM-CSF: enhancement of pregnancy specific interferon production, ovine trophoblast protein-1. Endocrinology 1993; 132:1869-1871.

134. Imakawa K, Tamura K, McGuire WJ, Khan S, Harbison LA, Stanga JP, Helmer SD, Christenson RK. Effect of interleukin-3 on ovine trophoblast interferon during early conceptus development. Endocrine 1995; 3:511-517.

135. Michael DD, Alvarez IM, Ocon OM, Powell AM, Talbot NC, Johnson SE, Ealy AD. Fibroblast growth factor-2 is expressed by the bovine uterus and stimulates interferon-tau production in bovine trophectoderm. Endocrinology 2006; 147:3571-3579. 136. Zhao FQ, Keating AF. Functional properties and genomics of glucose transporters. Curr Genomics 2007; 8:113-128.

137. Hosios AM, Hecht VC, Danai LV, Johnson MO, Rathmell JC, Steinhauser ML, Manalis SR, Vander Heiden MG. Amino Acids Rather than Glucose Account for the Majority of Cell Mass in Proliferating Mammalian Cells. Dev Cell 2016; 36:540-549. 138. Gao H, Wu G, Spencer TE, Johnson GA, Bazer FW. Select nutrients in the ovine uterine lumen. ii. glucose transporters in the uterus and peri-implantation conceptuses. Biol Reprod 2009; 80:94-104. 
139. Gao H, Wu G, Spencer TE, Johnson GA, Li X, Bazer FW. Select nutrients in the ovine uterine lumen. I. Amino acids, glucose, and ions in uterine lumenal flushings of cyclic and pregnant ewes. Biol Reprod 2009; 80:86-93.

140. Krisher RL, Prather RS. A role for the Warburg effect in preimplantation embryo development: metabolic modification to support rapid cell proliferation. Mol Reprod Dev $2012 ; 79: 311-320$.

141. Mullen MP, Bazer FW, Wu G, Parr MH, Evans AC, Crowe MA, Diskin MG. Effects of systemic progesterone during the early luteal phase on the availabilities of amino acids and glucose in the bovine uterine lumen. Reprod Fertil Dev 2014; 26:282292.

142. Rognstad R. Rate-limiting steps in metabolic pathways. J Biol Chem 1979; $254: 1875-1878$.

143. Li K, Ying M, Feng D, Du J, Chen S, Dan B, Wang C, Wang Y. Fructose-1,6bisphosphatase is a novel regulator of Wnt/beta-Catenin pathway in breast cancer. Biomed Pharmacother 2016; 84:1144-1149.

144. Zimmer DB, Magnuson MA. Immunohistochemical localization of phosphoenolpyruvate carboxykinase in adult and developing mouse tissues. J Histochem Cytochem 1990; 38:171-178.

145. Yanez AJ, Nualart F, Droppelmann C, Bertinat R, Brito M, Concha, II, Slebe JC. Broad expression of fructose-1,6-bisphosphatase and phosphoenolpyruvate carboxykinase provide evidence for gluconeogenesis in human tissues other than liver and kidney. J Cell Physiol 2003; 197:189-197. 
146. Moore SG, McCabe MS, Green JC, Newsom EM, Lucy MC. The transcriptome of the endometrium and placenta is associated with pregnancy development but not lactation status in dairy cows. Biol Reprod 2017; 97:18-31.

147. Szablewski L. Distribution of glucose transporters in renal diseases. J Biomed Sci $2017 ; 24: 64$.

148. Thorens B, Mueckler M. Glucose transporters in the 21st Century. Am J Physiol Endocrinol Metab 2010; 298:E141-145.

149. Feng L, Frommer WB. Structure and function of SemiSWEET and SWEET sugar transporters. Trends Biochem Sci 2015; 40:480-486.

150. Wright EM. Glucose transport families SLC5 and SLC50. Mol Aspects Med $2013 ; 34: 183-196$.

151. Frolova AI, Moley KH. Glucose transporters in the uterus: an analysis of tissue distribution and proposed physiological roles. Reproduction 2011; 142:211-220.

152. Franca MR, Mesquita FS, Lopes E, Pugliesi G, Van Hoeck V, Chiaratti MR, Membrive CB, Papa PC, Binelli M. Modulation of periovulatory endocrine profiles in beef cows: consequences for endometrial glucose transporters and uterine fluid glucose levels. Domest Anim Endocrinol 2015; 50:83-90.

153. Folmes CD, Terzic A. Metabolic determinants of embryonic development and stem cell fate. Reprod Fertil Dev 2014; 27:82-88.

154. Vander Heiden MG, Cantley LC, Thompson CB. Understanding the Warburg effect: the metabolic requirements of cell proliferation. Science 2009; 324:1029-1033. 155. Ingledew WJ, Poole RK. The respiratory chains of Escherichia coli. Microbiol Rev 1984; 48:222-271. 
156. Warburg O, Wind F, Negelein E. The Metabolism of Tumors in the Body. J Gen Physiol 1927; 8:519-530.

157. Warburg O. On the origin of cancer cells. Science 1956; 123:309-314.

158. DeBerardinis RJ, Mancuso A, Daikhin E, Nissim I, Yudkoff M, Wehrli S, Thompson CB. Beyond aerobic glycolysis: transformed cells can engage in glutamine metabolism that exceeds the requirement for protein and nucleotide synthesis. Proc Natl Acad Sci U S A 2007; 104:19345-19350.

159. Wise DR, Thompson CB. Glutamine addiction: a new therapeutic target in cancer. Trends Biochem Sci 2010; 35:427-433.

160. Eagle H. Nutrition needs of mammalian cells in tissue culture. Science 1955; 122:501-514.

161. Forman HJ, Zhang H, Rinna A. Glutathione: overview of its protective roles, measurement, and biosynthesis. Mol Aspects Med 2009; 30:1-12.

162. Chen Y, Shertzer HG, Schneider SN, Nebert DW, Dalton TP. Glutamate cysteine ligase catalysis: dependence on ATP and modifier subunit for regulation of tissue glutathione levels. J Biol Chem 2005; 280:33766-33774.

163. Choi YK, Park KG. Targeting Glutamine Metabolism for Cancer Treatment. Biomol Ther (Seoul) 2018; 26:19-28.

164. Zitka O, Skalickova S, Gumulec J, Masarik M, Adam V, Hubalek J, Trnkova L, Kruseova J, Eckschlager T, Kizek R. Redox status expressed as GSH:GSSG ratio as a marker for oxidative stress in paediatric tumour patients. Oncol Lett 2012; 4:1247-1253. 165. Chai YC, Ashraf SS, Rokutan K, Johnston RB, Jr., Thomas JA. S-thiolation of individual human neutrophil proteins including actin by stimulation of the respiratory 
burst: evidence against a role for glutathione disulfide. Arch Biochem Biophys 1994; 310:273-281.

166. Bazer FW, Wang X, Johnson GA, Wu G. Select nutrients and their effects on conceptus development in mammals. Anim Nutr 2015; 1:85-95.

167. Chen LQ, Cheung LS, Feng L, Tanner W, Frommer WB. Transport of sugars. Annu Rev Biochem 2015; 84:865-894.

168. Carracedo A, Cantley LC, Pandolfi PP. Cancer metabolism: fatty acid oxidation in the limelight. Nat Rev Cancer 2013; 13:227-232.

169. Wathes DC, Abayasekara DR, Aitken RJ. Polyunsaturated fatty acids in male and female reproduction. Biol Reprod 2007; 77:190-201.

170. Beall JR. Uterine lipid metabolism--a review of the literature. Comp Biochem Physiol B 1972; 42:175-195.

171. Brinsfield TH, Hawk HW. Control by progesterone of the concentration of lipid droplets in epithelial cells of the sheep endometrium. J Anim Sci 1973; 36:919-922.

172. Ribeiro ES. Symposium review: Lipids as regulators of conceptus development: Implications for metabolic regulation of reproduction in dairy cattle. J Dairy Sci 2018; $101: 3630-3641$.

173. Boshier DP, Fairclough RJ, Holloway H. Assessment of sheep blastocyst effects on neutral lipids in the uterine caruncular epithelium. J Reprod Fertil 1987; 79:569-573. 174. Gottlicher M, Widmark E, Li Q, Gustafsson JA. Fatty acids activate a chimera of the clofibric acid-activated receptor and the glucocorticoid receptor. Proc Natl Acad Sci U S A 1992; 89:4653-4657.

175. Berger J, Moller DE. The mechanisms of action of PPARs. Annu Rev Med 2002; $53: 409-435$. 
176. Xu HE, Lambert MH, Montana VG, Parks DJ, Blanchard SG, Brown PJ, Sternbach DD, Lehmann JM, Wisely GB, Willson TM, Kliewer SA, Milburn MV. Molecular recognition of fatty acids by peroxisome proliferator-activated receptors. Mol Cell 1999; 3:397-403.

177. Georgiadi A, Kersten S. Mechanisms of gene regulation by fatty acids. Adv Nutr $2012 ; 3: 127-134$.

178. Grygiel-Gorniak B. Peroxisome proliferator-activated receptors and their ligands: nutritional and clinical implications--a review. Nutr J 2014; 13:17.

179. Moraes JGN, Behura SK, Geary TW, Hansen PJ, Neibergs HL, Spencer TE. Uterine influences on conceptus development in fertility-classified animals. Proc Natl Acad Sci U S A 2018; 115:E1749-E1758.

180. Blanchard PG, Festuccia WT, Houde VP, St-Pierre P, Brule S, Turcotte V, Cote M, Bellmann K, Marette A, Deshaies Y. Major involvement of mTOR in the PPARgamma-induced stimulation of adipose tissue lipid uptake and fat accretion. J Lipid Res 2012; 53:1117-1125.

181. Hegarty BD, Furler SM, Oakes ND, Kraegen EW, Cooney GJ. Peroxisome proliferator-activated receptor (PPAR) activation induces tissue-specific effects on fatty acid uptake and metabolism in vivo--a study using the novel PPARalpha/gamma agonist tesaglitazar. Endocrinology 2004; 145:3158-3164.

182. Picard F, Auwerx J. PPAR(gamma) and glucose homeostasis. Annu Rev Nutr 2002; 22:167-197.

183. Rosen ED, Spiegelman BM. PPARgamma : a nuclear regulator of metabolism, differentiation, and cell growth. J Biol Chem 2001; 276:37731-37734. 
184. Chirala SS, Chang H, Matzuk M, Abu-Elheiga L, Mao J, Mahon K, Finegold M, Wakil SJ. Fatty acid synthesis is essential in embryonic development: fatty acid synthase null mutants and most of the heterozygotes die in utero. Proc Natl Acad Sci U S A 2003; 100:6358-6363.

185. Begriche K, Massart J, Robin MA, Borgne-Sanchez A, Fromenty B. Druginduced toxicity on mitochondria and lipid metabolism: mechanistic diversity and deleterious consequences for the liver. J Hepatol 2011; 54:773-794.

186. Woclawek-Potocka I, Kowalczyk-Zieba I, Skarzynski DJ. Lysophosphatidic acid action during early pregnancy in the cow: in vivo and in vitro studies. J Reprod Dev 2010; 56:411-420.

187. Guillou H, Zadravec D, Martin PG, Jacobsson A. The key roles of elongases and desaturases in mammalian fatty acid metabolism: Insights from transgenic mice. Prog Lipid Res 2010; 49:186-199.

188. Sassa T, Kihara A. Metabolism of very long-chain Fatty acids: genes and pathophysiology. Biomol Ther (Seoul) 2014; 22:83-92.

189. Ribeiro ES, Santos JE, Thatcher WW. Role of lipids on elongation of the preimplantation conceptus in ruminants. Reproduction 2016; 152:R115-126.

190. van der Vusse GJ. Albumin as fatty acid transporter. Drug Metab Pharmacokinet 2009; 24:300-307.

191. Barta E, Sideman S, Bassingthwaighte JB. Facilitated diffusion and membrane permeation of fatty acid in albumin solutions. Ann Biomed Eng 2000; 28:331-345.

192. Brooks K, Burns GW, Moraes JG, Spencer TE. Analysis of the Uterine Epithelial and Conceptus Transcriptome and Luminal Fluid Proteome During the Peri-Implantation Period of Pregnancy in Sheep. Biol Reprod 2016; 95:88. 
193. Feingold KR, Grunfeld C. Introduction to Lipids and Lipoproteins. In: De Groot LJ, Chrousos G, Dungan K, Feingold KR, Grossman A, Hershman JM, Koch C, Korbonits M, McLachlan R, New M, Purnell J, Rebar R, et al. (eds.), Endotext. South Dartmouth (MA); 2000.

194. Argraves WS, Morales CR. Immunolocalization of cubilin, megalin, apolipoprotein J, and apolipoprotein A-I in the uterus and oviduct. Mol Reprod Dev 2004; 69:419-427.

195. Koch JM, Ramadoss J, Magness RR. Proteomic profile of uterine luminal fluid from early pregnant ewes. J Proteome Res 2010; 9:3878-3885.

196. Khera AV, Kathiresan S. Genetics of coronary artery disease: discovery, biology and clinical translation. Nat Rev Genet 2017; 18:331-344.

197. Glatz JF, Luiken JJ, Bonen A. Membrane fatty acid transporters as regulators of lipid metabolism: implications for metabolic disease. Physiol Rev 2010; 90:367-417. 198. Bauersachs S, Ulbrich SE, Reichenbach HD, Reichenbach M, Buttner M, Meyer HH, Spencer TE, Minten M, Sax G, Winter G, Wolf E. Comparison of the effects of early pregnancy with human interferon, alpha 2 (IFNA2), on gene expression in bovine endometrium. Biol Reprod 2012; 86:46.

199. Record M, Silvente-Poirot S, Poirot M, Wakelam MJO. Extracellular vesicles: lipids as key components of their biogenesis and functions. J Lipid Res 2018; 59:13161324.

200. Yang Y, Hong Y, Cho E, Kim GB, Kim IS. Extracellular vesicles as a platform for membrane-associated therapeutic protein delivery. J Extracell Vesicles 2018; $7: 1440131$. 
201. Burns GW, Brooks KE, Spencer TE. Extracellular Vesicles Originate from the Conceptus and Uterus During Early Pregnancy in Sheep. Biol Reprod 2016; 94:56.

202. Trajkovic K, Hsu C, Chiantia S, Rajendran L, Wenzel D, Wieland F, Schwille P, Brugger B, Simons M. Ceramide triggers budding of exosome vesicles into multivesicular endosomes. Science 2008; 319:1244-1247.

203. Komar CM. Peroxisome proliferator-activated receptors (PPARs) and ovarian function--implications for regulating steroidogenesis, differentiation, and tissue remodeling. Reprod Biol Endocrinol 2005; 3:41.

204. Spencer TE, Forde N, Dorniak P, Hansen TR, Romero JJ, Lonergan P. Conceptus-derived prostaglandins regulate gene expression in the endometrium prior to pregnancy recognition in ruminants. Reproduction 2013; 146:377-387.

205. Charpigny G, Reinaud P, Tamby JP, Creminon C, Guillomot M. Cyclooxygenase2 unlike cyclooxygenase-1 is highly expressed in ovine embryos during the implantation period. Biol Reprod 1997; 57:1032-1040.

206. Lim H, Dey SK. A novel pathway of prostacyclin signaling-hanging out with nuclear receptors. Endocrinology 2002; 143:3207-3210.

207. Kliewer SA, Sundseth SS, Jones SA, Brown PJ, Wisely GB, Koble CS, Devchand P, Wahli W, Willson TM, Lenhard JM, Lehmann JM. Fatty acids and eicosanoids regulate gene expression through direct interactions with peroxisome proliferatoractivated receptors alpha and gamma. Proc Natl Acad Sci U S A 1997; 94:4318-4323. 208. Forman BM, Chen J, Evans RM. Hypolipidemic drugs, polyunsaturated fatty acids, and eicosanoids are ligands for peroxisome proliferator-activated receptors alpha and delta. Proc Natl Acad Sci U S A 1997; 94:4312-4317. 
209. Narumiya S, Sugimoto Y, Ushikubi F. Prostanoid receptors: structures, properties, and functions. Physiol Rev 1999; 79:1193-1226.

210. Woodward DF, Jones RL, Narumiya S. International Union of Basic and Clinical Pharmacology. LXXXIII: classification of prostanoid receptors, updating 15 years of progress. Pharmacol Rev 2011; 63:471-538.

211. Khan AH, Carson RJ, Nelson SM. Prostaglandins in labor--a translational approach. Front Biosci 2008; 13:5794-5809.

212. Coleman RA, Smith WL, Narumiya S. International Union of Pharmacology classification of prostanoid receptors: properties, distribution, and structure of the receptors and their subtypes. Pharmacol Rev 1994; 46:205-229.

213. Ide T, Egan K, Bell-Parikh LC, FitzGerald GA. Activation of nuclear receptors by prostaglandins. Thromb Res 2003; 110:311-315.

214. Lim H, Gupta RA, Ma WG, Paria BC, Moller DE, Morrow JD, DuBois RN, Trzaskos JM, Dey SK. Cyclo-oxygenase-2-derived prostacyclin mediates embryo implantation in the mouse via PPARdelta. Genes Dev 1999; 13:1561-1574.

215. Forman BM, Tontonoz P, Chen J, Brun RP, Spiegelman BM, Evans RM. 15Deoxy-delta 12, 14-prostaglandin J2 is a ligand for the adipocyte determination factor PPAR gamma. Cell 1995; 83:803-812.

216. Kliewer SA, Lenhard JM, Willson TM, Patel I, Morris DC, Lehmann JM. A prostaglandin $\mathrm{J} 2$ metabolite binds peroxisome proliferator-activated receptor gamma and promotes adipocyte differentiation. Cell 1995; 83:813-819.

217. Brooks K, Spencer TE. Biological roles of interferon tau (IFNT) and type I IFN receptors in elongation of the ovine conceptus. Biol Reprod 2015; 92:47. 
218. Dorniak P, Bazer FW, Spencer TE. Prostaglandins regulate conceptus elongation and mediate effects of interferon tau on the ovine uterine endometrium. Biol Reprod $2011 ; 84: 1119-1127$.

219. Dorniak P, Welsh TH, Jr., Bazer FW, Spencer TE. Endometrial HSD11B1 and cortisol regeneration in the ovine uterus: effects of pregnancy, interferon tau, and prostaglandins. Biol Reprod 2012; 86:124.

220. Oakley RH, Cidlowski JA. The biology of the glucocorticoid receptor: new signaling mechanisms in health and disease. J Allergy Clin Immunol 2013; 132:10331044.

221. Dorniak P, Welsh TH, Jr., Bazer FW, Spencer TE. Cortisol and interferon tau regulation of endometrial function and conceptus development in female sheep. Endocrinology 2013; 154:931-941.

222. Chen SJ, Liu YL, Sytwu HK. Immunologic regulation in pregnancy: from mechanism to therapeutic strategy for immunomodulation. Clin Dev Immunol 2012; 2012:258391.

223. Clemmons DR. Role of insulin-like growth factor binding proteins in controlling IGF actions. Mol Cell Endocrinol 1998; 140:19-24.

224. Harvey MB, Kaye PL. Insulin-like growth factor-1 stimulates growth of mouse preimplantation embryos in vitro. Mol Reprod Dev 1992; 31:195-199.

225. Gleeson LM, Chakraborty C, McKinnon T, Lala PK. Insulin-like growth factorbinding protein 1 stimulates human trophoblast migration by signaling through alpha 5 beta 1 integrin via mitogen-activated protein Kinase pathway. J Clin Endocrinol Metab $2001 ; 86: 2484-2493$ 
226. Kaczynski P, Kowalewski MP, Waclawik A. Prostaglandin F2alpha promotes angiogenesis and embryo-maternal interactions during implantation. Reproduction 2016; 151:539-552.

227. Hoeben A, Landuyt B, Highley MS, Wildiers H, Van Oosterom AT, De Bruijn EA. Vascular endothelial growth factor and angiogenesis. Pharmacol Rev 2004; 56:549580.

228. Zhang Y, Daaka Y. PGE2 promotes angiogenesis through EP4 and PKA Cgamma pathway. Blood 2011; 118:5355-5364.

229. Hamilton GS, Kennedy TG. Uterine vascular changes after unilateral intrauterine infusion of indomethacin and prostaglandin E2 to rats sensitized for the decidual cell reaction. Biol Reprod 1994; 50:757-764.

230. Majed BH, Khalil RA. Molecular mechanisms regulating the vascular prostacyclin pathways and their adaptation during pregnancy and in the newborn. Pharmacol Rev 2012; 64:540-582.

231. Arosh JA, Banu SK, Kimmins S, Chapdelaine P, Maclaren LA, Fortier MA. Effect of interferon-tau on prostaglandin biosynthesis, transport, and signaling at the time of maternal recognition of pregnancy in cattle: evidence of polycrine actions of prostaglandin E2. Endocrinology 2004; 145:5280-5293.

232. Diskin MG, Morris DG. Embryonic and early foetal losses in cattle and other ruminants. Reprod Domest Anim 2008; 43 Suppl 2:260-267.

233. Santos JEP, Thatcher WW, Chebel RC, Cerri RLA, Galvao KN. The effect of embryonic death rates in cattle on the efficacy of estrus synchronization programs. Animal Reproduction Science 2004; 82:513-535. 
234. Hansen PJ, Block J. Towards an embryocentric world: the current and potential uses of embryo technologies in dairy production. Reproduction, Fertility and Development 2003; 16:1-14.

235. Diskin MG, Murphy JJ, Sreenan JM. Embryo survival in dairy cows managed under pastoral conditions. Animal reproduction science 2006; 96:297-311.

236. Diskin MG, Parr MH, Morris DG. Embryo death in cattle: an update. Reprod Fertil Dev 2011; 24:244-251.

237. Berg DK, Van Leeuwen J, Beaumont S, Berg M, Pfeffer PL. Embryo loss in cattle between Days 7 and 16 of pregnancy. Theriogenology 2010; 73:250-260.

238. Diskin MG, Sreenan JM. Fertilization and embryonic mortality rates in beef heifers after artificial insemination. Journal of Reproduction and Fertility 1980; 59:463468.

239. Roche JF, Bolandl MP, McGeady TA. Reproductive wastage following artificial insemination of heifers. The Veterinary Record 1981; 109:401-404.

240. Chebel RC, Santos JE, Reynolds JP, Cerri RL, Juchem SO, Overton M. Factors affecting conception rate after artificial insemination and pregnancy loss in lactating dairy cows. Animal Reproduction Science 2004; 84:239-255.

241. Moore K, Thatcher WW. Major advances associated with reproduction in dairy cattle. Journal of Dairy Science 2006; 89:1254-1266.

242. Looney CR, Nelson JS, Schneider HJ, Forrest DW. Improving fertility in beef cow recipients. Theriogenology 2006; 65:201-209.

243. McMillan WH. Statistical models predicting embryo survival to term in cattle after embryo transfer. Theriogenology 1998; 50:1053-1070. 
244. Miravet-Valenciano JA, Rincon-Bertolin A, Vilella F, Simon C. Understanding and improving endometrial receptivity. Curr Opin Obstet Gynecol 2015; 27:187-192. 245. Spencer TE, Hansen TR. Implantation and Establishment of Pregnancy in Ruminants. Adv Anat Embryol Cell Biol 2015; 216:105-135.

246. Betteridge KJ, Flechon J-E. The anatomy and physiology of pre-attachment bovine embryos. Theriogenology 1988; 29:155-187.

247. Betteridge KJ, Eaglesome MD, Randall GCB, Mitchell D. Collection, description and transfer of embryos from cattle 10--16 days after oestrus. Journal of reproduction and fertility $1980 ; 59: 205-216$.

248. Guillomot M, REINAUD P, Charpigny G, Martal J. Embryo-uterine interactions during early stages of pregnancy in domestic mammals. Reproduction Nutrition Développement 1988; 28:1629-1648.

249. Fléchon J-E, Guillomot M, CHARLIER M, FLÉCHON B, Martal J. Experimental studies on the elongation of the ewe blastocyst. Reproduction Nutrition Développement $1986 ; 26: 1017-1024$

250. Satterfield MC, Bazer FW, Spencer TE. Progesterone regulation of preimplantation conceptus growth and galectin 15 (LGALS15) in the ovine uterus. Biol Reprod 2006; 75:289-296.

251. Spencer TE, Forde N, Lonergan P. The role of progesterone and conceptusderived factors in uterine biology during early pregnancy in ruminants. J Dairy Sci 2015. 252. FoRdE N, LoNERgAN P. Transcriptomic analysis of the bovine endometrium: what is required to establish uterine receptivity to implantation in cattle? Journal of Reproduction and Development 2012; 58:189-195. 
253. Forde N, Carter F, Spencer TE, Bazer FW, Sandra O, Mansouri-Attia N, Okumu LA, McGettigan PA, Mehta JP, McBride R, O'Gaora P, Roche JF, et al. Conceptusinduced changes in the endometrial transcriptome: how soon does the cow know she is pregnant? Biol Reprod 2011; 85:144-156.

254. Forde N, Spencer TE, Bazer FW, Song G, Roche JF, Lonergan P. Effect of pregnancy and progesterone concentration on expression of genes encoding for transporters or secreted proteins in the bovine endometrium. Physiol Genomics 2010; 41:53-62.

255. Allison Gray C, Bartol FF, Taylor KM, Wiley AA, Ramsey WS, Ott TL, Bazer FW, Spencer TE. Ovine uterine gland knock-out model: effects of gland ablation on the estrous cycle. Biol Reprod 2000; 62:448-456.

256. Gray CA, Taylor KM, Ramsey WS, Hill JR, Bazer FW, Bartol FF, Spencer TE. Endometrial glands are required for preimplantation conceptus elongation and survival. Biol Reprod 2001; 64:1608-1613.

257. Farin CE, Imakawa K, Roberts RM. In situ localization of mRNA for the interferon, ovine trophoblast protein-1, during early embryonic development of the sheep. Molecular Endocrinology 1989; 3:1099-1107.

258. Farin CE, Imakawa K, Hansen TR, McDonnell JJ, Murphy CN, Farin PW, Roberts RM. Expression of trophoblastic interferon genes in sheep and cattle. Biology of Reproduction 1990; 43:210-218.

259. Roberts RM, Chen Y, Ezashi T, Walker AM. Interferons and the maternalconceptus dialog in mammals. Semin Cell Dev Biol 2008; 19:170-177. 
260. Spencer TE, Sandra O, Wolf E. Genes involved in conceptus-endometrial interactions in ruminants: insights from reductionism and thoughts on holistic approaches. Reproduction 2008; 135:165-179.

261. Thatcher WW, Guzeloglu A, Mattos R, Binelli M, Hansen TR, Pru JK. Uterineconceptus interactions and reproductive failure in cattle. Theriogenology 2001; 56:14351450.

262. Lonergan P. State-of-the-art embryo technologies in cattle. Soc Reprod Fertil Suppl 2007; 64:315-325.

263. Lonergan P, Forde N, Spencer T. Role of progesterone in embryo development in cattle. Reproduction, Fertility and Development 2016; 28:66-74.

264. Bauersachs S, Mitko K, Ulbrich SE, Blum H, Wolf E. Transcriptome studies of bovine endometrium reveal molecular profiles characteristic for specific stages of estrous cycle and early pregnancy. Exp Clin Endocrinol Diabetes 2008; 116:371-384.

265. Ulbrich SE, Groebner AE, Bauersachs S. Transcriptional profiling to address molecular determinants of endometrial receptivity--lessons from studies in livestock species. Methods 2013; 59:108-115.

266. McMillan WH, Donnison MJ. Understanding maternal contributions to fertility in recipient cattle: development of herds with contrasting pregnancy rates. Animal reproduction science 1999; 57:127-140.

267. Peterson AJ, Lee R-F. Improving successful pregnancies after embryo transfer. Theriogenology 2003; 59:687-697. 268. Parrish JJ, Krogenaes A, Susko-Parrish JL. Effect of bovine sperm separation by either swim-up or Percoll method on success of in vitro fertilization and early embryonic development. Theriogenology 1995; 44:859-869. 
269. Sakatani M, Alvarez NV, Takahashi M, Hansen PJ. Consequences of physiological heat shock beginning at the zygote stage on embryonic development and expression of stress response genes in cattle. Journal of dairy science 2012; 95:30803091.

270. Bellow RA, Staigmiller RB, Wilson JM, Phelps DA, Darling A. Use of bovine FSH for superovulation and embryo production in beef heifers. Theriogenology 1991; 35:1069-1082.

271. Pohler KG, Pereira MHC, Lopes FR, Lawrence JC, Keisler DH, Smith MF, Vasconcelos JLM, Green JA. Circulating concentrations of bovine pregnancy-associated glycoproteins and late embryonic mortality in lactating dairy herds. Journal of dairy science 2015 .

272. Martin M. Cutadapt removes adapter sequences from high-throughput sequencing reads. EMBnet. journal 2011; 17:pp-10.

273. Liao Y, Smyth GK, Shi W. The Subread aligner: fast, accurate and scalable read mapping by seed-and-vote. Nucleic acids research 2013; 41:e108-e108.

274. Liao Y, Smyth GK, Shi W. featureCounts: an efficient general purpose program for assigning sequence reads to genomic features. Bioinformatics 2013:btt656.

275. Zhou X, Lindsay H, Robinson MD. Robustly detecting differential expression in RNA sequencing data using observation weights. Nucleic acids research 2014; 42:e91e91.

276. Whitacre LK, Tizioto PC, Kim J, Sonstegard TS, Schroeder SG, Alexander LJ, Medrano JF, Schnabel RD, Taylor JF, Decker JE. What's in your next-generation sequence data? An exploration of unmapped DNA and RNA sequence reads from the bovine reference individual. BMC Genomics 2015; 16:1114. 
277. Sreenan JM, Diskin MG. Factors affecting pregnancy rate following embryo transfer in the cow. Theriogenology 1987; 27:99-113.

278. Garrett JE, Geisert RD, Zavy MT, Morgan GL. Evidence for maternal regulation of early conceptus growth and development in beef cattle. Journal of Reproduction and Fertility 1988; 84:437-446.

279. Forde N, Beltman ME, Duffy GB, Duffy P, Mehta JP, O'Gaora P, Roche JF, Lonergan P, Crowe MA. Changes in the endometrial transcriptome during the bovine estrous cycle: effect of low circulating progesterone and consequences for conceptus elongation. Biol Reprod 2011; 84:266-278.

280. Forde N, Carter F, Fair T, Crowe MA, Evans ACO, Spencer TE, Bazer FW, McBride R, Boland MP, O'Gaora P, Lonergan P, Roche JF. Progesterone-regulated changes in endometrial gene expression contribute to advanced conceptus development in cattle. Biol Reprod 2009; 81:784-794.

281. Minten MA, Bilby TR, Bruno RGS, Allen CC, Madsen CA, Wang Z, Sawyer JE, Tibary A, Neibergs HL, Geary TW, Bauersachs S, Spencer TE. Effects of fertility on gene expression and function of the bovine endometrium. PLoS One 2013; 8:e69444. 282. Parr MH, Crowe MA, Lonergan P, Evans ACO, Rizos D, Diskin MG. Effect of exogenous progesterone supplementation in the early luteal phase post-insemination on pregnancy per artificial insemination in Holstein--Friesian cows. Animal reproduction science 2014; 150:7-14.

283. Beltman ME, Lonergan P, Diskin MG, Roche JF, Crowe MA. Effect of progesterone supplementation in the first week post conception on embryo survival in beef heifers. Theriogenology 2009; 71:1173-1179. 
284. Santos JE, Thatcher WW, Pool L, Overton MW. Effect of human chorionic gonadotropin on luteal function and reproductive performance of high-producing lactating Holstein dairy cows. J Anim Sci 2001; 79:2881-2894.

285. López-Gatius F, Santolaria P, Martino A, Delétang F, De Rensis F. The effects of $\mathrm{GnRH}$ treatment at the time of $\mathrm{AI}$ and 12 days later on reproductive performance of high producing dairy cows during the warm season in northeastern Spain. Theriogenology 2006; 65:820-830.

286. Nascimento AB, Bender RW, Souza AH, Ayres H, Araujo RR, Guenther JN, Sartori R, Wiltbank MC. Effect of treatment with human chorionic gonadotropin on day 5 after timed artificial insemination on fertility of lactating dairy cows. J Dairy Sci 2013; 96:2873-2882.

287. Mann GE, Fray MD, Lamming GE. Effects of time of progesterone supplementation on embryo development and interferon- $\tau$ production in the cow. The Veterinary Journal 2006; 171:500-503.

288. Lonergan P, Woods A, Fair T, Carter F, Rizos D, Ward F, Quinn K, Evans A. Effect of embryo source and recipient progesterone environment on embryo development in cattle. Reproduction, Fertility and Development 2007; 19:861-868.

289. Wiltbank MC, Souza AH, Carvalho PD, Bender RW, Nascimento AB. Improving fertility to timed artificial insemination by manipulation of circulating progesterone concentrations in lactating dairy cattle. Reproduction, Fertility and Development 2011; 24:238-243.

290. Salilew-Wondim D, Hölker M, Rings F, Ghanem N, Ulas-Cinar M, Peippo J, Tholen E, Looft C, Schellander K, Tesfaye D. Bovine pretransfer endometrium and 
embryo transcriptome fingerprints as predictors of pregnancy success after embryo transfer. Physiol Genomics 2010; 42:201-218.

291. Moore SG, Pryce JE, Hayes BJ, Chamberlain AJ, Kemper KE, Berry DP, McCabe M, Cormican P, Lonergan P, Fair T, Butler ST. Differentially Expressed Genes in Endometrium and Corpus Luteum of Holstein Cows Selected for High and Low Fertility Are Enriched for Sequence Variants Associated with Fertility. Biology of Reproduction 2015; 94:19-19.

292. Diamond G, Zasloff M, Eck H, Brasseur M, Maloy WL, Bevins CL. Tracheal antimicrobial peptide, a cysteine-rich peptide from mammalian tracheal mucosa: peptide isolation and cloning of a cDNA. Proceedings of the National Academy of Sciences $1991 ; 88: 3952-3956$.

293. DeSouza MM, Surveyor GA, Price RE, Julian J, Kardon R, Zhou X, Gendler S, Hilkens J, Carson DD. MUC1/episialin: a critical barrier in the female reproductive tract. J Reprod Immunol 1999; 45:127-158.

294. Walker CG, Meier S, Littlejohn MD, Lehnert K, Roche JR, Mitchell MD. Modulation of the maternal immune system by the pre-implantation embryo. BMC Genomics 2010; 11:474.

295. French LR, Northey DL. Inhibitory effect of the bovine conceptus on lymphocyte stimulation. J Anim Sci 1983; 57:456-465.

296. Skopets B, Li J, Thatcher WW, Roberts RM, Hansen PJ. Inhibition of lymphocyte proliferation by bovine trophoblast protein-1 (type I trophoblast interferon) and bovine interferon-alpha I1. Vet Immunol Immunopathol 1992; 34:81-96.

297. Fair T. The contribution of the maternal immune system to the establishment of pregnancy in cattle. Front Immunol 2015; 6:7. 
298. Vander Wielen AL, King GJ. Intraepithelial lymphocytes in the bovine uterus during the oestrous cycle and early gestation. J Reprod Fertil 1984; 70:457-462. 299. Low BG, Hansen PJ, Drost M, Gogolin-Ewens KJ. Expression of major histocompatibility complex antigens on the bovine placenta. J Reprod Fertil 1990; 90:235-243.

300. Oliveira LJ, Mansouri-Attia N, Mansourri-Attia N, Fahey AG, Browne J, Forde N, Roche JF, Lonergan P, Fair T. Characterization of the Th profile of the bovine endometrium during the oestrous cycle and early pregnancy. PLoS One 2013; 8:e75571. 301. Leung ST, Derecka K, Mann GE, Flint AP, Wathes DC. Uterine lymphocyte distribution and interleukin expression during early pregnancy in cows. J Reprod Fertil $2000 ; 119: 25-33$.

302. Mansouri-Attia N, Oliveira LJ, Forde N, Fahey AG, Browne JA, Roche JF, Sandra O, Reinaud P, Lonergan P, Fair T. Pivotal role for monocytes/macrophages and dendritic cells in maternal immune response to the developing embryo in cattle. Biol Reprod 2012; 87:123.

303. Santos JEP, Thatcher WW, Chebel RC, Cerri RLA, Galvão KN. The effect of embryonic death rates in cattle on the efficacy of estrus synchronization programs. Anim Reprod Sci 2004; 82-83:513-535.

304. Perkel KJ, Tscherner A, Merrill C, Lamarre J, Madan P. The ART of selecting the best embryo: A review of early embryonic mortality and bovine embryo viability assessment methods. Mol Reprod Dev 2015; 82:822-838.

305. Roberts RM. The place of farm animal species in the new genomics world of reproductive biology. Biol Reprod 2001; 64:409-417. 
306. Hyde KJ, Schust DJ. Genetic considerations in recurrent pregnancy loss. Cold Spring Harb Perspect Med 2015; 5:a023119.

307. Diskin MG, Parr MH, Morris DG. Embryo death in cattle: an update. Reprod Fertil Dev 2011; 24:244-251.

308. Rubio C, Bellver J, Rodrigo L, Bosch E, Mercader A, Vidal C, De los Santos MJ, Giles J, Labarta E, Domingo J, Crespo J, Remohi J, et al. Preimplantation genetic screening using fluorescence in situ hybridization in patients with repetitive implantation failure and advanced maternal age: two randomized trials. Fertil Steril 2013; 99:14001407.

309. Looney CR, Nelson JS, Schneider HJ, Forrest DW. Improving fertility in beef cow recipients. Theriogenology 2006; 65:201-209.

310. Ferraz PA, Burnley C, Karanja J, Viera-Neto A, Santos JE, Chebel RC, Galvao KN. Factors affecting the success of a large embryo transfer program in Holstein cattle in a commercial herd in the southeast region of the United States. Theriogenology 2016; $86: 1834-1841$.

311. Spencer TE, Forde N, Lonergan P. Insights into conceptus elongation and establishment of pregnancy in ruminants. Reprod Fertil Dev 2016; 29:84-100.

312. Hoelker M, Held E, Salilew-Wondim D, Schellander K, Tesfaye D. Molecular signatures of bovine embryo developmental competence. Reprod Fertil Dev 2013; 26:2236.

313. Miravet-Valenciano JA, Rincon-Bertolin A, Vilella F, Simon C. Understanding and improving endometrial receptivity. Curr Opin Obstet Gynecol 2015; 27:187-192. 314. McMillan WH. Expected pregnancy rate in recipient cattle, sheep and goats derived using a model incorporating embryo and maternal contributions to embryo 
survival. Proceedings of the New Zealand Society for Animal Production 1997; 57:218221.

315. Spencer TE, Forde N, Lonergan P. The role of progesterone and conceptusderived factors in uterine biology during early pregnancy in ruminants. J Dairy Sci 2016; 99:5941-5950.

316. Betteridge KJ, Flechon JE. The anatomy and physiology of pre-attachment bovine embryos. Theriogenology 1988; 29:155-187.

317. Forde N, Spencer TE, Bazer FW, Song G, Roche JF, Lonergan P. Effect of pregnancy and progesterone concentration on expression of genes encoding for transporters or secreted proteins in the bovine endometrium. Physiol Genomics 2010; 41:53-62.

318. Farin CE, Imakawa K, Hansen TR, McDonnell JJ, Murphy CN, Farin PW, Roberts RM. Expression of trophoblastic interferon genes in sheep and cattle. Biol Reprod 1990; 43:210-218.

319. Shorten PR, Ledgard AM, Donnison M, Pfeffer PL, McDonald RM, Berg DK. A mathematical model of the interaction between bovine blastocyst developmental stage and progesterone-stimulated uterine factors on differential embryonic development observed on Day 15 of gestation. J Dairy Sci 2017:pii: S0022-0302(0017)30987-30986. 320. Spencer TE, Hansen TR. Implantation and Establishment of Pregnancy in Ruminants. Adv Anat Embryol Cell Biol 2015; 216:105-135.

321. Roberts RM, Chen Y, Ezashi T, Walker AM. Interferons and the maternalconceptus dialog in mammals. Seminars in Cell \& Developmental Biology 2008; 19:170177. 
322. Thatcher WW, Guzeloglu A, Mattos R, Binelli M, Hansen TR, Pru JK. Uterineconceptus interactions and reproductive failure in cattle. Theriogenology 2001; 56:14351450.

323. Lonergan P. State-of-the-art embryo technologies in cattle. Soc Reprod Fertil Suppl 2007; 64:315-325.

324. Bauersachs S, Mitko K, Ulbrich SE, Blum H, Wolf E. Transcriptome studies of bovine endometrium reveal molecular profiles characteristic for specific stages of estrous cycle and early pregnancy. Exp Clin Endocrinol Diabetes 2008; 116:371-384.

325. Ulbrich SE, Groebner AE, Bauersachs S. Transcriptional profiling to address molecular determinants of endometrial receptivity--lessons from studies in livestock species. Methods 2013; 59:108-115.

326. McMillan WH, Donnison MJ. Understanding maternal contributions to fertility in recipient cattle: development of herds with contrasting pregnancy rates. Anim Reprod Sci $1999 ; 57: 127-140$.

327. Peterson AJ, Lee RS. Improving successful pregnancies after embryo transfer. Theriogenology 2003; 59:687-697.

328. Geary TW, Burns GW, Moraes JG, Moss JI, Denicol AC, Dobbs KB, Ortega MS, Hansen PJ, Wehrman ME, Neibergs H, O'Neil E, Behura S, et al. Identification of beef heifers with superior uterine capacity for pregnancy. Biol Reprod 2016; 95:47.

329. Tarca AL, Draghici S, Khatri P, Hassan SS, Mittal P, Kim JS, Kim CJ, Kusanovic JP, Romero R. A novel signaling pathway impact analysis. Bioinformatics 2009; 25:7582. 
330. Barnwell CV, Farin PW, Ashwell CM, Farmer WT, Galphin SP, Jr., Farin CE. Differences in mRNA populations of short and long bovine conceptuses on Day 15 of gestation. Mol Reprod Dev 2016; 83:424-441.

331. Bult CJ, Eppig JT, Kadin JA, Richardson JE, Blake JA, Mouse Genome Database G. The Mouse Genome Database (MGD): mouse biology and model systems. Nucleic Acids Res 2008; 36:D724-728.

332. Scrucca L, Fop M, Murphy TB, Raftery AE. mclust 5: Clustering, Classification and Density Estimation Using Gaussian Finite Mixture Models. R J 2016; 8:289-317. 333. Ramilowski JA, Goldberg T, Harshbarger J, Kloppmann E, Lizio M, Satagopam VP, Itoh M, Kawaji H, Carninci P, Rost B, Forrest AR. A draft network of ligandreceptor-mediated multicellular signalling in human. Nat Commun 2015; 6:7866. 334. Capra JA, Williams AG, Pollard KS. ProteinHistorian: tools for the comparative analysis of eukaryote protein origin. PLoS Comput Biol 2012; 8:e1002567.

335. Diskin MG, Morris DG. Embryonic and early foetal losses in cattle and other ruminants. Reprod Domest Anim 2008; 43 Suppl 2:260-267.

336. Bormann JM, Totir LR, Kachman SD, Fernando RL, Wilson DE. Pregnancy rate and first-service conception rate in Angus heifers. J Anim Sci 2006; 84:2022-2025. 337. Azzam SM, Kinder JE, Nielsen MK. Conception rate at first insemination in beef cattle: effects of season, age and previous reproductive performance. J Anim Sci 1989; 67:1405-1410.

338. Chebel RC, Santos JE, Reynolds JP, Cerri RL, Juchem SO, Overton M. Factors affecting conception rate after artificial insemination and pregnancy loss in lactating dairy cows. Anim Reprod Sci 2004; 84:239-255. 
339. O'Hara L, Forde N, Kelly AK, Lonergan P. Effect of bovine blastocyst size at embryo transfer on day 7 on conceptus length on day 14: can supplementary progesterone rescue small embryos? Theriogenology 2014; 81:1123-1128.

340. Shorten PR, Ledgard AM, Donnison M, Pfeffer PL, McDonald RM, Berg DK. A mathematical model of the interaction between bovine blastocyst developmental stage and progesterone-stimulated uterine factors on differential embryonic development observed on Day 15 of gestation. J Dairy Sci 2017; 101:736-751.

341. King GJ, Atkinson BA, Robertson HA. Implantation and early placentation in domestic ungulates. J Reprod Fertil Suppl 1982; 31:17-30.

342. Imakawa K, Bai R, Fujiwara H, Ideta A, Aoyagi Y, Kusama K. Continuous model of conceptus implantation to the maternal endometrium. J Endocrinol 2017; 233:R53R65.

343. Neto ACA, Pereira FTV, Santos TC, Ambrosio CE, Leiser R, Miglino MA. Morpho-physical recording of bovine conceptus (Bos indicus) and placenta from days 20 to 70 of pregnancy. Reproduction in Domestic Animals 2010; 45:760-772.

344. Heyman Y, Chavatte-Palmer P, LeBourhis D, Camous S, Vignon X, Renard JP. Frequency and occurrence of late-gestation losses from cattle cloned embryos. Biol Reprod 2002; 66:6-13.

345. Biase FH, Rabel C, Guillomot M, Hue I, Andropolis K, Olmstead CA, Oliveira R, Wallace R, Le Bourhis D, Richard C, Campion E, Chaulot-Talmon A, et al. Massive dysregulation of genes involved in cell signaling and placental development in cloned cattle conceptus and maternal endometrium. Proc Natl Acad Sci U S A 2016; 113:1449214501. 
346. Bauersachs S, Wolf E. Uterine responses to the preattachment embryo in domestic ungulates: recognition of pregnancy and preparation for implantation. Annu Rev Anim Biosci 2015; 3:489-511.

347. Forde N, Mehta JP, Minten M, Crowe MA, Roche JF, Spencer TE, Lonergan P. Effects of low progesterone on the endometrial transcriptome in cattle. Biol Reprod 2012; $87: 124$

348. Carter F, Forde N, Duffy P, Wade M, Fair T, Crowe MA, Evans AC, Kenny DA, Roche JF, Lonergan P. Effect of increasing progesterone concentration from Day 3 of pregnancy on subsequent embryo survival and development in beef heifers. Reprod Fertil Dev 2008; 20:368-375.

349. Minten MA, Bilby TR, Bruno RG, Allen CC, Madsen CA, Wang Z, Sawyer JE, Tibary A, Neibergs HL, Geary TW, Bauersachs S, Spencer TE. Effects of fertility on gene expression and function of the bovine endometrium. PLoS One 2013; 8:e69444. 350. Matsuyama S, Kojima T, Kato S, Kimura K. Relationship between quantity of IFNT estimated by IFN-stimulated gene expression in peripheral blood mononuclear cells and bovine embryonic mortality after AI or ET. Reprod Biol Endocrinol 2012; 10:21. 351. Rueda BR, Naivar KA, George EM, Austin KJ, Francis H, Hansen TR. Recombinant interferon-tau regulates secretion of two bovine endometrial proteins. J Interferon Res 1993; 13:303-309.

352. Robinson RS, Mann GE, Lamming GE, Wathes DC. Expression of oxytocin, oestrogen and progesterone receptors in uterine biopsy samples throughout the oestrous cycle and early pregnancy in cows. Reproduction 2001; 122:965-979.

353. Salilew-Wondim D, Holker M, Rings F, Ghanem N, Ulas-Cinar M, Peippo J, Tholen E, Looft C, Schellander K, Tesfaye D. Bovine pretransfer endometrium and 
embryo transcriptome fingerprints as predictors of pregnancy success after embryo transfer. Physiological genomics 2010; 42:201-218.

354. Vasudevan S, Kamat MM, Walusimbi SS, Pate JL, Ott TL. Effects of early pregnancy on uterine lymphocytes and endometrial expression of immune-regulatory molecules in dairy heifers. Biol Reprod 2017; 97:104-118.

355. Koot YEM, Macklon NS. Embryo implantation: biology, evaluation, and enhancement. Current Opinion in Obstetrics \& Gynecology 2013; 25:274-279. 356. Salamonsen LA, Edgell T, Rombauts LJ, Stephens AN, Robertson DM, Rainczuk A, Nie G, Hannan NJ. Proteomics of the human endometrium and uterine fluid: a pathway to biomarker discovery. Fertil Steril 2013; 99:1086-1092. 357. Evans GE, Martinez-Conejero JA, Phillipson GT, Simon C, McNoe LA, Sykes PH, Horcajadas JA, Lam EY, Print CG, Sin IL, Evans JJ. Gene and protein expression signature of endometrial glandular and stromal compartments during the window of implantation. Fertil Steril 2012; 97:1365-1373 e1361-1362.

358. Dorniak P, Bazer FW, Spencer TE. Physiology and Endocrinology Symposium: biological role of interferon tau in endometrial function and conceptus elongation. $\mathrm{J}$ Anim Sci 2013; 91:1627-1638.

359. Brooks KE, Burns G, Spencer TE. Conceptus elongation in ruminants: roles of progesterone, prostaglandin, interferon tau and cortisol. Journal of Animal Science and Biotechnology 2014; 5:53.

360. El-Sayed A, Hoelker M, Rings F, Salilew D, Jennen D, Tholen E, Sirard MA, Schellander K, Tesfaye D. Large-scale transcriptional analysis of bovine embryo biopsies in relation to pregnancy success after transfer to recipients. Physiol Genomics 2006; 28:84-96. 
361. Bazer FW, Johnson GA, Wu G. Amino acids and conceptus development during the peri-implantation period of pregnancy. Adv Exp Med Biol 2015; 843:23-52.

362. Lea RG, Sandra O. Immunoendocrine aspects of endometrial function and implantation. Reproduction 2007; 134:389-404.

363. Cerri RL, Thompson IM, Kim IH, Ealy AD, Hansen PJ, Staples CR, Li JL, Santos JE, Thatcher WW. Effects of lactation and pregnancy on gene expression of endometrium of Holstein cows at day 17 of the estrous cycle or pregnancy. J Dairy Sci 2012; 95:56575675.

364. Klein C, Bauersachs S, Ulbrich SE, Einspanier R, Meyer HH, Schmidt SE, Reichenbach HD, Vermehren M, Sinowatz F, Blum H, Wolf E. Monozygotic twin model reveals novel embryo-induced transcriptome changes of bovine endometrium in the preattachment period. Biol Reprod 2006; 74:253-264.

365. Guillomot M. Changes in extracellular matrix components and cytokeratins in the endometrium during goat implantation. Placenta 1999; 20:339-345.

366. Pfarrer C, Hirsch P, Guillomot M, Leiser R. Interaction of integrin receptors with extracellular matrix is involved in trophoblast giant cell migration in bovine placentomes. Placenta 2003; 24:588-597.

367. Kizaki K, Ushizawa K, Takahashi T, Yamada O, Todoroki J, Sato T, Ito A, Hashizume K. Gelatinase (MMP-2 and -9) expression profiles during gestation in the bovine endometrium. Reprod Biol Endocrinol 2008; 6:66.

368. Mishra B, Kizaki K, Sato T, Ito A, Hashizume K. The role of extracellular matrix metalloproteinase inducer (EMMPRIN) in the regulation of bovine endometrial cell functions. Biol Reprod 2012; 87:149. 
369. Mishra B, Kizaki K, Koshi K, Ushizawa K, Takahashi T, Hosoe M, Sato T, Ito A, Hashizume K. Expression of extracellular matrix metalloproteinase inducer (EMMPRIN) and its expected roles in the bovine endometrium during gestation. Domest Anim Endocrinol 2012; 42:63-73.

370. Ulbrich SE, Meyer SU, Zitta K, Hiendleder S, Sinowatz F, Bauersachs S, Buttner M, Frohlich T, Arnold GJ, Richenbach HD, Wolf E, Meyer HH. Bovine endometrial metallopeptidases MMP14 and MMP2 and the metallopeptidase inhibitor TIMP2 participate in maternal preparation of pregnancy. Mol Cell Endocrinol 2011; 332:48-57. 371. Spencer TE, Johnson GA, Bazer FW, Burghardt RC. Implantation mechanisms: insights from the sheep. Reproduction 2004; 128:657-668.

372. Murphy CR. The cytoskeleton of uterine epithelial cells: a new player in uterine receptivity and the plasma membrane transformation. Hum Reprod Update 1995; 1:567580.

373. Bainbridge SA, Minhas A, Whiteley KJ, Qu D, Sled JG, Kingdom JC, Adamson SL. Effects of reduced Gcm1 expression on trophoblast morphology, fetoplacental vascularity, and pregnancy outcomes in mice. Hypertension 2012; 59:732-739.

374. Bazer FW, Spencer TE, Johnson GA, Burghardt RC, Wu G. Comparative aspects of implantation. Reproduction 2009; 138:195-209.

375. Biase FH, Rabel C, Guillomot M, Sandra O, Andropolis K, Olmstead C, Oliveira R, Wallace R, Le Bourhis D, Richard C, Campion E, Chaulot-Talmon A, et al. Changes in WNT signaling-related gene expression associated with development and cloning in bovine extra-embryonic and endometrial tissues during the peri-implantation period. Mol Reprod Dev 2013; 80:977-987. 
376. Hayashi K, Burghardt RC, Bazer FW, Spencer TE. WNTs in the ovine uterus: potential regulation of periimplantation ovine conceptus development. Endocrinology 2007; 148:3496-3506.

377. Sandra O, Constant F, Vitorino Carvalho A, Eozenou C, Valour D, Mauffre V, Hue I, Charpigny G. Maternal organism and embryo biosensoring: insights from ruminants. J Reprod Immunol 2015; 108:105-113.

378. Mansouri-Attia N, Sandra O, Aubert J, Degrelle S, Everts RE, Giraud-Delville C, Heyman Y, Galio L, Hue I, Yang X, Tian XC, Lewin HA, et al. Endometrium as an early sensor of in vitro embryo manipulation technologies. Proc Natl Acad Sci U S A 2009; 106:5687-5692.

379. Chakrabarty A, MacLean JA, 2nd, Hughes AL, Roberts RM, Green JA. Rapid evolution of the trophoblast kunitz domain proteins (TKDPs)-a multigene family in ruminant ungulates. J Mol Evol 2006; 63:274-282.

380. Wetendorf M, DeMayo FJ. The progesterone receptor regulates implantation, decidualization, and glandular development via a complex paracrine signaling network. Mol Cell Endocrinol 2012; 357:108-118.

381. Tu Z, Ran H, Zhang S, Xia G, Wang B, Wang H. Molecular determinants of uterine receptivity. Int J Dev Biol 2014; 58:147-154.

382. Cha J, Sun X, Dey SK. Mechanisms of implantation: strategies for successful pregnancy. Nat Med 2012; 18:1754-1767.

383. Garrido-Gomez T, Dominguez F, Quinonero A, Diaz-Gimeno P, Kapidzic M, Gormley M, Ona K, Padilla-Iserte P, McMaster M, Genbacev O, Perales A, Fisher SJ, et al. Defective decidualization during and after severe preeclampsia reveals a possible maternal contribution to the etiology. Proc Natl Acad Sci U S A 2017. 
384. Bauersachs S, Ulbrich SE, Zakhartchenko V, Minten M, Reichenbach M, Reichenbach HD, Blum H, Spencer TE, Wolf E. The endometrium responds differently to cloned versus fertilized embryos. Proc Natl Acad Sci U S A 2009; 106:5681-5686. 385. Salilew-Wondim D, Fournier E, Hoelker M, Saeed-Zidane M, Tholen E, Looft C, Neuhoff C, Besenfelder U, Havlicek V, Rings F, Gagne D, Sirard MA, et al. Genomewide DNA methylation patterns of bovine blastocysts developed in vivo from embryos completed different dtages of development in vitro. PLoS One 2015; 10:e0140467. 386. Dobbs KB, Gagne D, Fournier E, Dufort I, Robert C, Block J, Sirard MA, Bonilla L, Ealy AD, Loureiro B, Hansen PJ. Sexual dimorphism in developmental programming of the bovine preimplantation embryo caused by colony-stimulating factor 2 . Biol Reprod 2014; 91:80.

387. Lucas ES, Dyer NP, Murakami K, Lee YH, Chan YW, Grimaldi G, Muter J, Brighton PJ, Moore JD, Patel G, Chan JK, Takeda S, et al. Loss of Endometrial Plasticity in Recurrent Pregnancy Loss. Stem Cells 2016; 34:346-356.

388. Walker CG, Littlejohn MD, Meier S, Roche JR, Mitchell MD. DNA methylation is correlated with gene expression during early pregnancy in Bos taurus. Physiol Genomics 2013; 45:276-286.

389. Ponsuksili S, Murani E, Schwerin M, Schellander K, Tesfaye D, Wimmers K. Gene expression and DNA-methylation of bovine pretransfer endometrium depending on its receptivity after in vitro-produced embryo transfer. PLoS One 2012; 7:e42402. 390. Voelkel SA, Hu YX. Use of ethylene glycol as a cryoprotectant for bovine embryos allowing direct transfer of frozen-thawed embryos to recipient females. Theriogenology 1992; 37:687-697. 
391. Kim D, Langmead B, Salzberg SL. HISAT: a fast spliced aligner with low memory requirements. Nat Methods 2015; 12:357-360.

392. Pertea M, Pertea GM, Antonescu CM, Chang TC, Mendell JT, Salzberg SL. StringTie enables improved reconstruction of a transcriptome from RNA-seq reads. Nat Biotechnol 2015; 33:290-295.

393. Zhou X, Lindsay H, Robinson MD. Robustly detecting differential expression in RNA sequencing data using observation weights. Nucleic Acids Res 2014; 42:e91. 394. Meyer PE, Lafitte F, Bontempi G. minet: A R/Bioconductor package for inferring large transcriptional networks using mutual information. BMC Bioinformatics 2008; 9:461.

395. Ihnatova I, Budinska E. ToPASeq: an R package for topology-based pathway analysis of microarray and RNA-Seq data. BMC Bioinformatics 2015; 16:350. 396. Chen J, Bardes EE, Aronow BJ, Jegga AG. ToppGene Suite for gene list enrichment analysis and candidate gene prioritization. Nucleic Acids Res 2009; 37:W305-311.

397. Galili T. dendextend: an R package for visualizing, adjusting and comparing trees of hierarchical clustering. Bioinformatics 2015; 31:3718-3720.

398. Clemente M, de La Fuente J, Fair T, Al Naib A, Gutierrez-Adan A, Roche JF, Rizos D, Lonergan P. Progesterone and conceptus elongation in cattle: a direct effect on the embryo or an indirect effect via the endometrium? Reproduction 2009; 138:507-517. 399. Koot YE, van Hooff SR, Boomsma CM, van Leenen D, Groot Koerkamp MJ, Goddijn M, Eijkemans MJ, Fauser BC, Holstege FC, Macklon NS. An endometrial gene expression signature accurately predicts recurrent implantation failure after IVF. Sci Rep 2016; 6:19411. 
400. Ruiz-Alonso M, Blesa D, Diaz-Gimeno P, Gomez E, Fernandez-Sanchez M, Carranza F, Carrera J, Vilella F, Pellicer A, Simon C. The endometrial receptivity array for diagnosis and personalized embryo transfer as a treatment for patients with repeated implantation failure. Fertil Steril 2013; 100:818-824.

401. Bauersachs S, Wolf E. Transcriptome analyses of bovine, porcine and equine endometrium during the pre-implantation phase. Anim Reprod Sci 2012; 134:84-94. 402. Ricciotti E, FitzGerald GA. Prostaglandins and inflammation. Arterioscler Thromb Vasc Biol 2011; 31:986-1000.

403. Funk CD. Prostaglandins and leukotrienes: advances in eicosanoid biology. Science 2001; 294:1871-1875.

404. Moon YA, Shah NA, Mohapatra S, Warrington JA, Horton JD. Identification of a mammalian long chain fatty acyl elongase regulated by sterol regulatory element-binding proteins. J Biol Chem 2001; 276:45358-45366.

405. Burns GW, Brooks KE, O'Neil EV, Hagen DE, Behura SK, Spencer TE. Progesterone effects on extracellular vesicles in the sheep uterus. Biol Reprod 2018; 98:612-622.

406. Moncada S. Eighth Gaddum Memorial Lecture. University of London Institute of Education, December 1980. Biological importance of prostacyclin. Br J Pharmacol 1982; $76: 3-31$

407. Salmon JA, Smith DR, Flower RJ, Moncada S, Vane JR. Further studies on the enzymatic conversion of prostaglandin endoperoxide into prostacyclin by porcine aorta microsomes. Biochim Biophys Acta 1978; 523:250-262. 
408. Chong J, Soufan O, Li C, Caraus I, Li S, Bourque G, Wishart DS, Xia J. MetaboAnalyst 4.0: towards more transparent and integrative metabolomics analysis. Nucleic Acids Res 2018; 46:W486-W494.

409. Xia J, Wishart DS. Web-based inference of biological patterns, functions and pathways from metabolomic data using MetaboAnalyst. Nat Protoc 2011; 6:743-760. 410. Xia J, Wishart DS. Metabolomic data processing, analysis, and interpretation using MetaboAnalyst. Curr Protoc Bioinformatics 2011; Chapter 14:Unit 1410.

411. Checa A, Bedia C, Jaumot J. Lipidomic data analysis: tutorial, practical guidelines and applications. Anal Chim Acta 2015; 885:1-16.

412. Leese HJ, Baumann CG, Brison DR, McEvoy TG, Sturmey RG. Metabolism of the viable mammalian embryo: quietness revisited. Mol Hum Reprod 2008; 14:667-672. 413. Gardner DK, Wale PL. Analysis of metabolism to select viable human embryos for transfer. Fertil Steril 2013; 99:1062-1072.

414. Wales RG, Waugh EE. Catabolic utilization of glucose by the sheep conceptus between days 13 and 19 of pregnancy. Reprod Fertil Dev 1993; 5:111-122.

415. Poyser NL. The control of prostaglandin production by the endometrium in relation to luteolysis and menstruation. Prostaglandins Leukot Essent Fatty Acids 1995; $53: 147-195$.

416. Lim H, Paria BC, Das SK, Dinchuk JE, Langenbach R, Trzaskos JM, Dey SK. Multiple female reproductive failures in cyclooxygenase 2-deficient mice. Cell 1997; 91:197-208.

417. Regan JW, Bailey TJ, Pepperl DJ, Pierce KL, Bogardus AM, Donello JE, Fairbairn CE, Kedzie KM, Woodward DF, Gil DW. Cloning of a novel human 
prostaglandin receptor with characteristics of the pharmacologically defined EP2 subtype. Mol Pharmacol 1994; 46:213-220.

418. Arosh JA, Banu SK, Chapdelaine P, Madore E, Sirois J, Fortier MA. Prostaglandin biosynthesis, transport, and signaling in corpus luteum: a basis for autoregulation of luteal function. Endocrinology 2004; 145:2551-2560.

419. Ochoa JC, Penagaricano F, Baez GM, Melo LF, Motta JC, Guerra AG, Meidan R, Ferreira JCP, Sartori R, Wiltbank MC. Mechanisms for rescue of CL during pregnancy: Gene expression in bovine CL following intrauterine pulses of Prostaglandins E1 and F2alpha. Biol Reprod 2017.

420. Dey I, Lejeune M, Chadee K. Prostaglandin E2 receptor distribution and function in the gastrointestinal tract. Br J Pharmacol 2006; 149:611-623.

421. Milne SA, Jabbour HN. Prostaglandin (PG) F(2alpha) receptor expression and signaling in human endometrium: role of $\mathrm{PGF}(2 \mathrm{alpha})$ in epithelial cell proliferation. $\mathrm{J}$ Clin Endocrinol Metab 2003; 88:1825-1832.

422. Kanai N, Lu R, Satriano JA, Bao Y, Wolkoff AW, Schuster VL. Identification and characterization of a prostaglandin transporter. Science 1995; 268:866-869.

423. Lu R, Kanai N, Bao Y, Schuster VL. Cloning, in vitro expression, and tissue distribution of a human prostaglandin transporter cDNA(hPGT). J Clin Invest 1996; 98:1142-1149.

424. Schuster VL. Prostaglandin transport. Prostaglandins Other Lipid Mediat 2002; 68-69:633-647.

425. Nakanishi T, Tamai I. Roles of Organic Anion Transporting Polypeptide 2A1 (OATP2A1/SLCO2A1) in Regulating the Pathophysiological Actions of Prostaglandins. AAPS J 2017; 20:13. 
426. Russel FG, Koenderink JB, Masereeuw R. Multidrug resistance protein 4 (MRP4/ABCC4): a versatile efflux transporter for drugs and signalling molecules. Trends Pharmacol Sci 2008; 29:200-207.

427. Seo H, Choi Y, Shim J, Yoo I, Ka H. Prostaglandin transporters ABCC4 and SLCO2A1 in the uterine endometrium and conceptus during pregnancy in pigs. Biol Reprod 2014; 90:100.

428. Cammas L, Reinaud P, Bordas N, Dubois O, Germain G, Charpigny G. Developmental regulation of prostacyclin synthase and prostacyclin receptors in the ovine uterus and conceptus during the peri-implantation period. Reproduction 2006; 131:917-927.

429. Moncada S, Gryglewski RJ, Bunting S, Vane JR. A lipid peroxide inhibits the enzyme in blood vessel microsomes that generates from prostaglandin endoperoxides the substance (prostaglandin X) which prevents platelet aggregation. Prostaglandins 1976; 12:715-737.

430. Dusting GJ, Moncada S, Vane JR. Prostacyclin (PGX) is the endogenous metabolite responsible for relaxation of coronary arteries induced by arachindonic acid. Prostaglandins 1977; 13:3-15.

431. Brooks KE, Burns GW, Spencer TE. Peroxisome proliferator activator receptor gamma (PPARG) regulates conceptus elongation in sheep. Biol Reprod 2015; 92:42. 432. Yoon M. The role of PPARalpha in lipid metabolism and obesity: focusing on the effects of estrogen on PPARalpha actions. Pharmacol Res 2009; 60:151-159.

433. Furuhashi M, Hotamisligil GS. Fatty acid-binding proteins: role in metabolic diseases and potential as drug targets. Nat Rev Drug Discov 2008; 7:489-503. 
434. Huynh H, Pollak M. Stabilization of mammary-derived growth inhibitor messenger RNA by antiestrogens. Clin Cancer Res 1997; 3:2151-2156.

435. Ufer C, Wang CC. The Roles of Glutathione Peroxidases during Embryo Development. Front Mol Neurosci 2011; 4:12.

436. Schaloske RH, Dennis EA. The phospholipase A2 superfamily and its group numbering system. Biochim Biophys Acta 2006; 1761:1246-1259.

437. Fuly AL, de Miranda AL, Zingali RB, Guimaraes JA. Purification and characterization of a phospholipase A2 isoenzyme isolated from Lachesis muta snake venom. Biochem Pharmacol 2002; 63:1589-1597.

438. Hui DY. Phospholipase A(2) enzymes in metabolic and cardiovascular diseases. Curr Opin Lipidol 2012; 23:235-240.

439. Murakami M, Taketomi Y, Miki Y, Sato H, Hirabayashi T, Yamamoto K. Recent progress in phospholipase A(2) research: from cells to animals to humans. Prog Lipid Res $2011 ; 50: 152-192$.

440. Agbaga MP, Mandal MN, Anderson RE. Retinal very long-chain PUFAs: new insights from studies on ELOVL4 protein. J Lipid Res 2010; 51:1624-1642.

441. Zadravec D, Tvrdik P, Guillou H, Haslam R, Kobayashi T, Napier JA, Capecchi MR, Jacobsson A. ELOVL2 controls the level of n-6 28:5 and 30:5 fatty acids in testis, a prerequisite for male fertility and sperm maturation in mice. J Lipid Res 2011; 52:245255.

442. Riezman H. The long and short of fatty acid synthesis. Cell 2007; 130:587-588.

443. Jump DB. Mammalian fatty acid elongases. Methods Mol Biol 2009; 579:375389. 
444. Stoffel W, Holz B, Jenke B, Binczek E, Gunter RH, Kiss C, Karakesisoglou I, Thevis M, Weber AA, Arnhold S, Addicks K. Delta6-desaturase (FADS2) deficiency unveils the role of omega3- and omega6-polyunsaturated fatty acids. EMBO J 2008; 27:2281-2292.

445. Glaser C, Heinrich J, Koletzko B. Role of FADS1 and FADS2 polymorphisms in polyunsaturated fatty acid metabolism. Metabolism 2010; 59:993-999.

446. Yu H, Zhao Z, Yu X, Li J, Lu C, Yang R. Bovine lipid metabolism related gene GPAM: Molecular characterization, function identification, and association analysis with fat deposition traits. Gene 2017; 609:9-18.

447. Li Z, Vance DE. Phosphatidylcholine and choline homeostasis. J Lipid Res 2008; 49:1187-1194.

448. Levy M, Futerman AH. Mammalian ceramide synthases. IUBMB Life 2010; 62:347-356.

449. Tirodkar TS, Lu P, Bai A, Scheffel MJ, Gencer S, Garrett-Mayer E, Bielawska A, Ogretmen B, Voelkel-Johnson C. Expression of Ceramide Synthase 6 Transcriptionally Activates Acid Ceramidase in a c-Jun N-terminal Kinase (JNK)-dependent Manner. J Biol Chem 2015; 290:13157-13167. 450. Slominski AT, Li W, Kim TK, Semak I, Wang J, Zjawiony JK, Tuckey RC. Novel activities of CYP11A1 and their potential physiological significance. J Steroid Biochem Mol Biol 2015; 151:25-37.

451. Seo T, Oelkers PM, Giattina MR, Worgall TS, Sturley SL, Deckelbaum RJ. Differential modulation of ACAT1 and ACAT2 transcription and activity by long chain free fatty acids in cultured cells. Biochemistry 2001; 40:4756-4762. 
452. van Meer G, Voelker DR, Feigenson GW. Membrane lipids: where they are and how they behave. Nat Rev Mol Cell Biol 2008; 9:112-124.

453. Meier S, Trewhella MA, Fairclough RJ, Jenkin G. Changes in uterine endometrial phospholipids and fatty acids throughout the oestrous cycle and early pregnancy in the ewe. Prostaglandins Leukot Essent Fatty Acids 1997; 57:341-349.

454. Burnum KE, Cornett DS, Puolitaival SM, Milne SB, Myers DS, Tranguch S, Brown HA, Dey SK, Caprioli RM. Spatial and temporal alterations of phospholipids determined by mass spectrometry during mouse embryo implantation. J Lipid Res 2009; $50: 2290-2298$.

455. Villarroya-Beltri C, Baixauli F, Mittelbrunn M, Fernandez-Delgado I, Torralba D, Moreno-Gonzalo O, Baldanta S, Enrich C, Guerra S, Sanchez-Madrid F. ISGylation controls exosome secretion by promoting lysosomal degradation of MVB proteins. Nat Commun 2016; 7:13588.

456. Simmet K, Zakhartchenko V, Philippou-Massier J, Blum H, Klymiuk N, Wolf E. OCT4/POU5F1 is required for NANOG expression in bovine blastocysts. Proc Natl Acad Sci U S A 2018; 115:2770-2775.

457. Pan GJ, Chang ZY, Scholer HR, Pei D. Stem cell pluripotency and transcription factor Oct4. Cell Res 2002; 12:321-329.

458. Ezashi T, Ghosh D, Roberts RM. Repression of Ets-2-induced transactivation of the tau interferon promoter by Oct-4. Mol Cell Biol 2001; 21:7883-7891. 459. Forde N, Bazer FW, Spencer TE, Lonergan P. 'Conceptualizing' the Endometrium: Identification of Conceptus-Derived Proteins During Early Pregnancy in Cattle. Biol Reprod 2015; 92:156. 
460. Groebner AE, Rubio-Aliaga I, Schulke K, Reichenbach HD, Daniel H, Wolf E, Meyer HH, Ulbrich SE. Increase of essential amino acids in the bovine uterine lumen during preimplantation development. Reproduction 2011; 141:685-695.

461. Hansen TR, Leaman DW, Cross JC, Mathialagan N, Bixby JA, Roberts RM. The genes for the trophoblast interferons and the related interferon-alpha II possess distinct 5'promoter and 3'-flanking sequences. J Biol Chem 1991; 266:3060-3067.

462. Tabb DL. What's driving false discovery rates? J Proteome Res 2008; 7:45-46.

463. Ribeiro ES, Monteiro APA, Bisinotto RS, Lima FS, Greco LF, Ealy AD, Thatcher WW, Santos JEP. Conceptus development and transcriptome at preimplantation stages in lactating dairy cows of distinct genetic groups and estrous cyclic statuses. J Dairy Sci 2016; 99:4761-4777.

464. Xia J, Mandal R, Sinelnikov IV, Broadhurst D, Wishart DS. MetaboAnalyst 2.0-a comprehensive server for metabolomic data analysis. Nucleic Acids Res 2012; 40:W127-133.

465. Gao H, Wu G, Spencer TE, Johnson GA, Bazer FW. Select nutrients in the ovine uterine lumen. III. Cationic amino acid transporters in the ovine uterus and periimplantation conceptuses. Biol Reprod 2009; 80:602-609.

466. Young VR, Ajami AM. Glutamine: the emperor or his clothes? J Nutr 2001; 131:2449S-2459S; discussion 2486S-2447S.

467. Laplante M, Sabatini DM. Regulation of mTORC1 and its impact on gene expression at a glance. J Cell Sci 2013; 126:1713-1719.

468. Cairns RA, Harris IS, Mak TW. Regulation of cancer cell metabolism. Nat Rev Cancer 2011; 11:85-95. 
469. Scalise M, Galluccio M, Console L, Pochini L, Indiveri C. The Human SLC7A5 (LAT1): The Intriguing Histidine/Large Neutral Amino Acid Transporter and Its Relevance to Human Health. Front Chem 2018; 6:243.

470. Wang X, Frank JW, Little DR, Dunlap KA, Satterfield MC, Burghardt RC, Hansen TR, Wu G, Bazer FW. Functional role of arginine during the peri-implantation period of pregnancy. I. Consequences of loss of function of arginine transporter SLC7A1 mRNA in ovine conceptus trophectoderm. FASEB J 2014; 28:2852-2863.

471. Hussain T, Tan B, Ren W, Rahu N, Kalhoro DH, Yin Y. Exploring polyamines: Functions in embryo/fetal development. Anim Nutr 2017; 3:7-10.

472. Wu G, Bazer FW, Davis TA, Kim SW, Li P, Marc Rhoads J, Carey Satterfield M, Smith SB, Spencer TE, Yin Y. Arginine metabolism and nutrition in growth, health and disease. Amino Acids 2009; 37:153-168.

473. Johnson GA, Burghardt RC, Bazer FW. Osteopontin: a leading candidate adhesion molecule for implantation in pigs and sheep. J Anim Sci Biotechnol 2014; 5:56. 474. Wang X, Johnson GA, Burghardt RC, Wu G, Bazer FW. Uterine Histotroph and Conceptus Development. II. Arginine and Secreted Phosphoprotein 1 Cooperatively Stimulate Migration and Adhesion of Ovine Trophectoderm Cells via Focal AdhesionMTORC2 Mediated Cytoskeleton Reorganization. Biol Reprod 2016; 95:71.

475. Datta D, Bhinge A, Chandran V. Lysine: Is it worth more? Cytotechnology 2001; $36: 3-32$.

476. Khan-Siddiqui L, Bamji MS. Lysine-carnitine conversion in normal and undernourished adult men-suggestion of a nonpeptidyl pathway. Am J Clin Nutr 1983; 37:93-98. 
477. Bene J, Hadzsiev K, Melegh B. Role of carnitine and its derivatives in the development and management of type 2 diabetes. Nutr Diabetes 2018; 8:8.

478. Rossi CR, Galzigna L, Alexandre A, Gibson DM. Oxidation of long chain fatty acids by rat liver mitochondria. J Biol Chem 1967; 242:2102-2110.

479. Calo LA, Pagnin E, Davis PA, Semplicini A, Nicolai R, Calvani M, Pessina AC. Antioxidant effect of L-carnitine and its short chain esters: relevance for the protection from oxidative stress related cardiovascular damage. Int J Cardiol 2006; 107:54-60. 480. Duranay M, Akay H, Yilmaz FM, Senes M, Tekeli N, Yucel D. Effects of Lcarnitine infusions on inflammatory and nutritional markers in haemodialysis patients. Nephrol Dial Transplant 2006; 21:3211-3214.

481. Robinson DP, Klein SL. Pregnancy and pregnancy-associated hormones alter immune responses and disease pathogenesis. Horm Behav 2012; 62:263-271.

482. Jauniaux E, Poston L, Burton GJ. Placental-related diseases of pregnancy: Involvement of oxidative stress and implications in human evolution. Hum Reprod Update $2006 ; 12: 747-755$.

483. Bauer BK, Isom SC, Spate LD, Whitworth KM, Spollen WG, Blake SM, Springer GK, Murphy CN, Prather RS. Transcriptional profiling by deep sequencing identifies differences in mRNA transcript abundance in in vivo-derived versus in vitro-cultured porcine blastocyst stage embryos. Biol Reprod 2010; 83:791-798.

484. Redel BK, Tessanne KJ, Spate LD, Murphy CN, Prather RS. Arginine increases development of in vitro-produced porcine embryos and affects the protein arginine methyltransferase-dimethylarginine dimethylaminohydrolase-nitric oxide axis. Reprod Fertil Dev 2015; 27:655-666. 
485. Chen PR, Redel BK, Spate LD, Ji T, Salazar SR, Prather RS. Glutamine supplementation enhances development of in vitro-produced porcine embryos and increases leucine consumption from the medium. Biol Reprod 2018.

486. Broer A, Wagner CA, Lang F, Broer S. The heterodimeric amino acid transporter 4F2hc/y+LAT2 mediates arginine efflux in exchange with glutamine. Biochem J 2000; 349 Pt 3:787-795.

487. Pochini L, Scalise M, Galluccio M, Indiveri C. Membrane transporters for the special amino acid glutamine: structure/function relationships and relevance to human health. Front Chem 2014; 2:61.

488. Finkelstein JD. Methionine metabolism in mammals. J Nutr Biochem 1990; 1:228-237.

489. Brosnan JT, Brosnan ME. The sulfur-containing amino acids: an overview. J Nutr 2006; 136:1636S-1640S.

490. Guyader-Joly C, Khatchadourian C, Menezo Y. Glycine and methionine transport by bovine embryos. Zygote 1997; 5:273-276.

491. Bonilla L, Luchini D, Devillard E, Hansen PJ. Methionine requirements for the preimplantation bovine embryo. J Reprod Dev 2010; 56:527-532.

492. Metayer S, Seiliez I, Collin A, Duchene S, Mercier Y, Geraert PA, Tesseraud S. Mechanisms through which sulfur amino acids control protein metabolism and oxidative status. J Nutr Biochem 2008; 19:207-215.

493. Chakravarthi S, Jessop CE, Bulleid NJ. The role of glutathione in disulphide bond formation and endoplasmic-reticulum-generated oxidative stress. EMBO Rep 2006; 7:271-275. 
494. Pallardo FV, Markovic J, Garcia JL, Vina J. Role of nuclear glutathione as a key regulator of cell proliferation. Mol Aspects Med 2009; 30:77-85.

495. Houten SM, Wanders RJ. A general introduction to the biochemistry of mitochondrial fatty acid beta-oxidation. J Inherit Metab Dis 2010; 33:469-477. 496. Ji H, Wang J, Guo J, Li Y, Lian S, Guo W, Yang H, Kong F, Zhen L, Guo L, Liu Y. Progress in the biological function of alpha-enolase. Anim Nutr 2016; 2:12-17. 497. Cavalcanti JH, Esteves-Ferreira AA, Quinhones CG, Pereira-Lima IA, NunesNesi A, Fernie AR, Araujo WL. Evolution and functional implications of the tricarboxylic acid cycle as revealed by phylogenetic analysis. Genome Biol Evol 2014; 6:2830-2848.

498. Youn HS, Kim TG, Kim MK, Kang GB, Kang JY, Lee JG, An JY, Park KR, Lee Y, Im YJ, Lee JH, Eom SH. Structural Insights into the Quaternary Catalytic Mechanism of Hexameric Human Quinolinate Phosphoribosyltransferase, a Key Enzyme in de novo NAD Biosynthesis. Sci Rep 2016; 6:19681.

499. Patil SB, Kodliwadmath MV, Kodliwadmath SM. Study of oxidative stress and enzymatic antioxidants in normal pregnancy. Indian J Clin Biochem 2007; 22:135-137. 500. Bhagwat SR, Redij T, Phalnikar K, Nayak S, Iyer S, Gadkar S, Chaudhari U, Kholkute SD, Sachdeva G. Cell surfactomes of two endometrial epithelial cell lines that differ in their adhesiveness to embryonic cells. Mol Reprod Dev 2014; 81:326-340.

501. Boutchueng-Djidjou M, Collard-Simard G, Fortier S, Hebert SS, Kelly I, Landry CR, Faure RL. The last enzyme of the de novo purine synthesis pathway 5aminoimidazole-4-carboxamide ribonucleotide formyltransferase/IMP cyclohydrolase (ATIC) plays a central role in insulin signaling and the Golgi/endosomes protein network. Mol Cell Proteomics 2015; 14:1079-1092. 
502. Iwanaga K, Nomura S, Ito T, Ikoma Y, Yamamoto E, Okada M, Itakura A, Kikkawa F, Tsujimoto M, Mizutani S. Placental leucine aminopeptidase/oxytocinase gene regulation by activator protein- 2 in BeWo cell model of human trophoblast differentiation. FEBS Lett 2003; 552:120-124.

503. Kim HY, Heo YS, Kim JH, Park MH, Moon J, Kim E, Kwon D, Yoon J, Shin D, Jeong EJ, Park SY, Lee TG, et al. Molecular basis for the local conformational rearrangement of human phosphoserine phosphatase. J Biol Chem 2002; 277:4665146658.

504. Kulecka M, Wierzbicka A, Paziewska A, Mikula M, Habior A, Janczyk W, Dabrowska M, Karczmarski J, Lazniewski M, Ginalski K, Czlonkowska A, Socha P, et al. A heterozygous mutation in GOT1 is associated with familial macro-aspartate aminotransferase. J Hepatol 2017; 67:1026-1030.

505. Ahn YH, Park S, Choi JJ, Park BK, Rhee KH, Kang E, Ahn S, Lee CH, Lee JS, Inn KS, Cho ML, Park SH, et al. Secreted tryptophanyl-tRNA synthetase as a primary defence system against infection. Nat Microbiol 2016; 2:16191.

506. Wang Y, Kavran JM, Chen Z, Karukurichi KR, Leahy DJ, Cole PA. Regulation of S-adenosylhomocysteine hydrolase by lysine acetylation. J Biol Chem 2014; 289:31361-31372.

507. Khurana S, Chakraborty S, Lam M, Liu Y, Su YT, Zhao X, Saleem MA, Mathieson PW, Bruggeman LA, Kao HY. Familial focal segmental glomerulosclerosis (FSGS)-linked alpha-actinin 4 (ACTN4) protein mutants lose ability to activate transcription by nuclear hormone receptors. J Biol Chem 2012; 287:12027-12035. 
508. Zhou GL, Zhang H, Wu H, Ghai P, Field J. Phosphorylation of the cytoskeletal protein CAP1 controls its association with cofilin and actin. J Cell Sci 2014; 127:50525065.

509. Morasso MI, Grinberg A, Robinson G, Sargent TD, Mahon KA. Placental failure in mice lacking the homeobox gene Dlx3. Proc Natl Acad Sci U S A 1999; 96:162-167. 510. Li S, Roberson MS. Dlx3 and GCM-1 functionally coordinate the regulation of placental growth factor in human trophoblast-derived cells. J Cell Physiol 2017; 232:2900-2914.

511. Chiu YH, Yang MR, Wang LJ, Chen MH, Chang GD, Chen H. New insights into the regulation of placental growth factor gene expression by the transcription factors GCM1 and DLX3 in human placenta. J Biol Chem 2018; 293:9801-9811.

512. Home P, Kumar RP, Ganguly A, Saha B, Milano-Foster J, Bhattacharya B, Ray S, Gunewardena S, Paul A, Camper SA, Fields PE, Paul S. Genetic redundancy of GATA factors in the extraembryonic trophoblast lineage ensures the progression of preimplantation and postimplantation mammalian development. Development 2017; 144:876-888.

513. Kawasaki H, Eckner R, Yao TP, Taira K, Chiu R, Livingston DM, Yokoyama KK. Distinct roles of the co-activators p300 and CBP in retinoic-acid-induced F9-cell differentiation. Nature 1998; 393:284-289.

514. Giordano A, Avantaggiati ML. p300 and CBP: partners for life and death. J Cell Physiol 1999; 181:218-230.

515. Goodman RH, Smolik S. CBP/p300 in cell growth, transformation, and development. Genes Dev 2000; 14:1553-1577. 
516. Kim MS, Min KS, Imakawa K. Regulation of Interferon-stimulated Gene (ISG)12, ISG15, and MX1 and MX2 by Conceptus Interferons (IFNTs) in Bovine Uterine Epithelial Cells. Asian-Australas J Anim Sci 2013; 26:795-803.

517. Antoniazzi AQ, Webb BT, Romero JJ, Ashley RL, Smirnova NP, Henkes LE, Bott RC, Oliveira JF, Niswender GD, Bazer FW, Hansen TR. Endocrine delivery of interferon tau protects the corpus luteum from prostaglandin F2 alpha-induced luteolysis in ewes. Biol Reprod 2013; 88:144.

518. Ashworth CJ, Bazer FW. Changes in ovine conceptus and endometrial function following asynchronous embryo transfer or administration of progesterone. Biol Reprod $1989 ; 40: 425-433$.

519. Oliveira JF, Henkes LE, Ashley RL, Purcell SH, Smirnova NP, Veeramachaneni DN, Anthony RV, Hansen TR. Expression of interferon (IFN)-stimulated genes in extrauterine tissues during early pregnancy in sheep is the consequence of endocrine IFNtau release from the uterine vein. Endocrinology 2008; 149:1252-1259.

520. Meyerholz MM, Mense K, Knaack H, Sandra O, Schmicke M. PregnancyInduced ISG-15 and MX-1 Gene Expression is Detected in the Liver of Holstein-Friesian Heifers During Late Peri-Implantation Period. Reprod Domest Anim 2016; 51:175-177. 


\section{VITA}

Joao Moraes grew up in Minas Gerais, a state located in the north of Southeastern Brazil, with his parents Adelio and Marcia, and his sister Nayara. Joao became interested in farm animals as a child, following the steps of his grandfather Joaquim, which was a dairy farmer. Due to his love for animals and exposure to farming growing up, Joao decided to pursue a career as a veterinarian, and was accepted as a student at the Universidade Federal de Uberlandia, Brazil in 2005. During veterinary school, Joao became fascinated with animal reproduction, and during his last academic year, he had the opportunity to participate in an externship at the University of Minnesota (UMN), under the supervision of Dr. Ricardo Chebel. Following graduation from Veterinary School in 2010, Dr. Chebel invited Joao to come back to the United States and work as a technician in his laboratory at the UMN. In 2011, Joao had the opportunity to further improve his training by pursuing a master's in Animal Sciences and a residency in Dairy Production Medicine, at the UMN. After completion of the masters in 2013 and residency in 2014, Joao joined the laboratory of Dr. Thomas Spencer at Washington State University (WSU), to work towards a PhD in reproductive biology with focus on uterine-conceptus interactions. In 2015, Dr. Spencer was recruited by the University of Missouri (MU), and Joao moved with him from Pullman to Columbia, where he completed his experiments and requirements to earn a $\mathrm{PhD}$ from MU. After completing his doctoral degree, Joao will stay at Mizzou and begin a postdoctoral position in the laboratory of Dr. Matthew Lucy, in the Division of Animal Sciences. In Dr. Lucy’s lab, Joao will continue his studies investigating uterine biology, with the overall goal of improving reproductive efficiency in livestock animals and humans. 